\title{
ÉVALUATION DE LA POLITIQUE D'INNOVATION SUR UN ECHANTILLON D' ENTREPRISES ALGERIENNES
}

\section{TESIS DOCTORAL}

Presentada por:

Nadia Ahmadouche

Dirigida por:

Dr. Jose Albors Garrigós

Dr. Samir Baha-Eddine Maliki

Dra. María Rosario Perelló Marín 



\section{RESUMÉ EXECUTIF}

L'objectif de cette thèse est d'evaluer la politique d'innovation dans les pays en développement par le système d'innovation. Cette démarche consite à rechercher les facteurs clefs qui déterminent l'innovation au sein des entreprises, en examinant l'adéquation entre les acteurs de l'innovation (universités, entreprises et ministères), les instruments politiques (les subventions de l'état, les lois et règlements), les activités d'innovation (recherche et développement interne et externe, formation, acquisition d'équipements) au sein des entreprises avec les résultats d'innovations. Un modèle reliant toutes ces dimensions est proposé. L'étude effectuée ne s'arrête pas seulement à l'analyse des principales dimensions mais va au-delà, en détectant chaque facteur clef apporté par chacune des composantes. Nous avons appliqué les techniques Parcial Least Square (PLS) pour tester le modèle théorique proposé à l'aide d'un questionnaire inspiré de l'enquête Canadienne de 2017 «Integrated Business Statistics Program (IBSP) Survey of Innovation and Business Strategy» et du questionnaire «The Community Innovation Survey» (CIS) de 2014. Un échantillon de 67 entreprises pharmaceutiques algériennes a été étudié. L'analyse du secteur montre que les politiques publiques algériennes à l'égard du secteur n'ont pas abordé la question de la promotion de l'innovation ni le développement de nouveaux produits pharmaceutiques spécifiques dans le pays. Les résultats obtenus confirment l'apport important de la formation et la recherche à l'innovation.

Mots clés. Système d'innovation, politique d'innovation du secteur pharmaceutique algerien, instrument politique, activité d'innovation, acteur d'innovation.

\section{EXECUTIVE SUMMARY}

The objective of this thesis is to assess innovation policy in developing countries by understanding the innovation system. This approach consists in researching the key factors that determine innovation within firms by examining the adequacy of innovation actors (university, firms and ministry), policy instruments (state subsidies, laws and regulations), innovation activities (internal and external research and development, training, equipment acquisition) within firms with the results of innovations. A model linking all these dimensions is proposed. The study carried out does not stop at the analysis of the main dimensions but goes beyond that by detecting each key factor contributed by each of the components. We applied Partial Least Square (PLS) techniques to test the proposed theoretical model using a questionnaire inspired by the 2017 Canadian "Integrated Business Statistics Program (IBSP) Survey of Innovation and Business Strategy" and the 2014 "The Community Innovation Survey" (CIS) questionnaire. A sample of 67 Algerian pharmaceutical companies was studied. The analysis of the sector shows that Algerian public policies towards the sector have not addressed the issue of promoting innovation or the development of new specific pharmaceutical 
products in the country. The results obtained confirm the important contribution of training and research to innovation.

Keywords. Innovation system, innovation policy of the Algerian pharmaceutical sector, political instrument, innovation activity, innovation actor

\section{RESUMEN EJECUTIVO}

El objetivo de esta tesis es evaluar la política de innovación en los países en desarrollo tratando de comprender el sistema de innovación. Para ello se investigaron los factores clave que determinan la innovación en las empresas examinando la idoneidad de los agentes de la innovación (universidad, empresas y ministerios), los instrumentos de política (subvenciones públicas, leyes y reglamentos) y las actividades de innovación (investigación y desarrollo interno y externo, capacitación, adquisición de equipo) en las empresas con resultados de innovación. Se ha propuesto un modelo que vincula todas estas dimensiones. Para ponerlo a prueba, aplicamos las técnicas de Partial Least Square (PLS) y nos inspiramos en la Encuesta de "Integrated Business Statistics Program (IBSP) Survey of Innovation and Business Strategy" del Canadá de 2017 y en el cuestionario de la Encuesta de "The Community Innovation Survey" (CIS) de 2014. El estudio abarcó una muestra de 67 empresas farmacéuticas argelinas. Este estudio no se limitó al análisis de las dimensiones principales, sino que fue más allá al detectar cada factor clave aportado por cada uno de los componentes. El análisis del sector farmacéutico muestra que las políticas públicas argelinas relativas a este sector no han abordado la cuestión de la promoción de la innovación o el desarrollo de nuevos productos farmacéuticos específicos en el país.Los resultados obtenidos confirman la importante contribución de la formación y la investigación a la innovación.

Palabras clave Sistema de innovación, política de innovación del sector farmacéutico argelino, instrumento político, actividad de innovación, actor de innovación.

\section{RESUM EXECUTIU}

L'objectiu d'esta tesi és avaluar la política d'innovació en els països en desenrotllament tractant de comprendre el sistema d'innovació. Per a això es van investigar els factors clau que determinen la innovació en les empreses examinant la idoneïtat dels agents de la innovació (universitat, empreses i ministeris), els instruments de política (subvencions públiques, lleis i reglaments) i les activitats d'innovació (investigació i desenrotllament intern i extern, capacitació, adquisició d'equip) en les empreses amb resultats d'innovació. S'ha proposat un model que vincula totes estes dimensions. S'ha proposat un model que vincula totes estes dimensions. Per a posar-ho a prova, apliquem les tècniques de Vaig partiral Least Square (PLS) i ens inspirem en l'Enquesta de "Integrated Business Statistics Program (IBSP) Survey of Innovation and Business Strategy"; del Canadà de 2017 i en el qüestionari de l'Enquesta de "The 
Community Innovation Survey" (CIS) de 2014. L'estudi va comprendre una mostra de 67 empreses farmacèutiques algerianes. Este estudi no es va limitar a l'anàlisi de les dimensions principals, sinó que va ser més enllà al detectar cada factor clau aportat per cada un dels components. L'anàlisi del sector farmacèutic mostra que les polítiques públiques algerianes relatives a este sector no han abordat la qüestió de la promoció de la innovació o el desenrotllament de nous productes farmacèutics específics en el país.Los resultats obtinguts confirmen la important contribució de la formació i la investigació a la innovació.

Paraules clau. Sistema d'innovació, política d'innovació del sector farmacèutic algerià, instrument polític, activitat d'innovació, actor d'innovació.

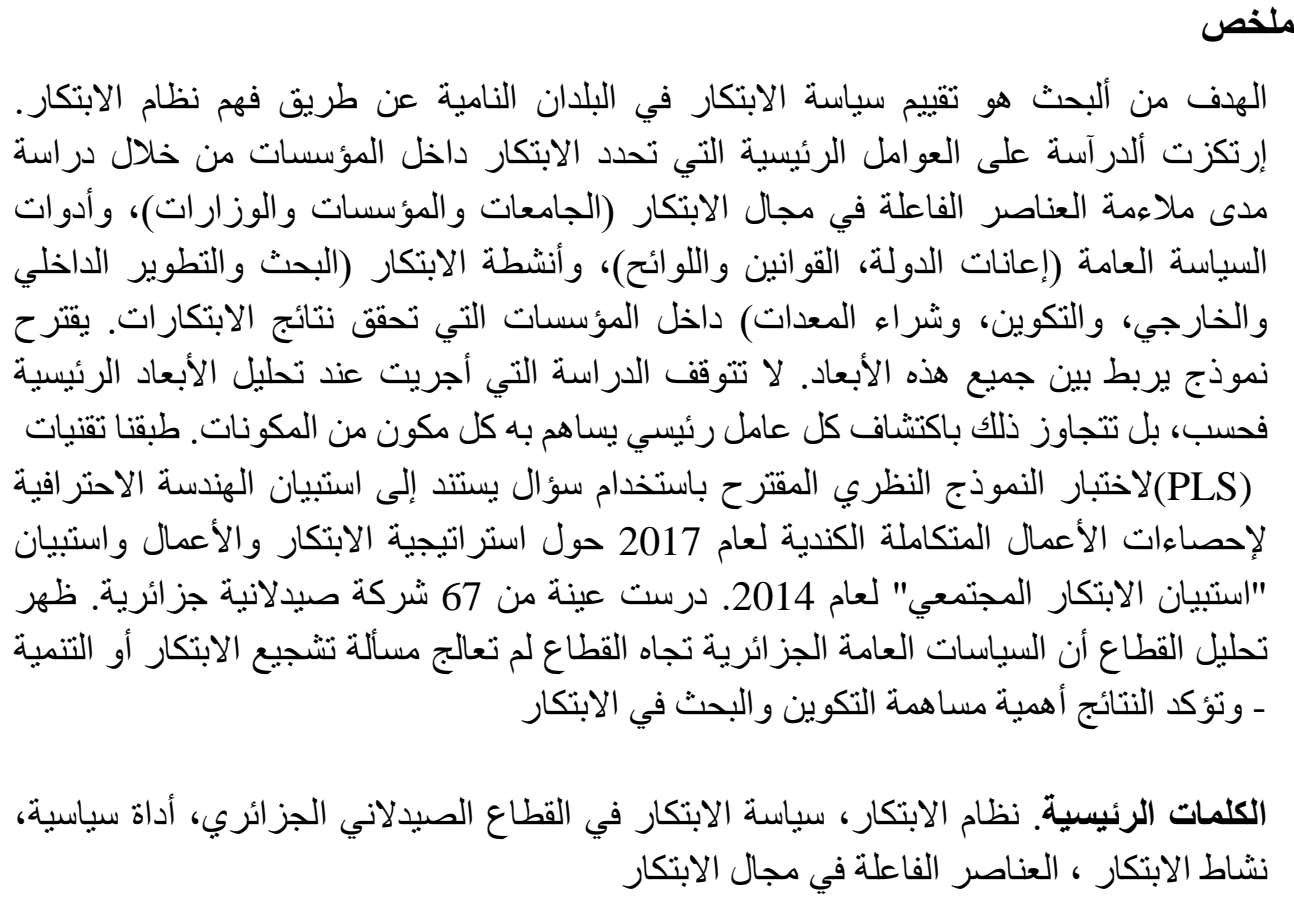


Evaluation de la politique d'innovation sur un échantillon d'entreprises algériennes 


\section{Table des matières}

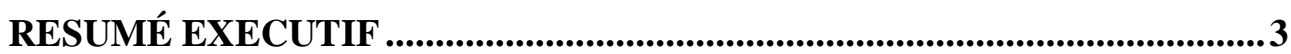

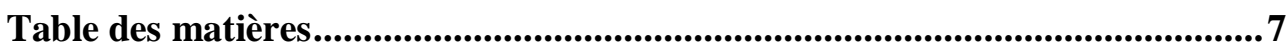

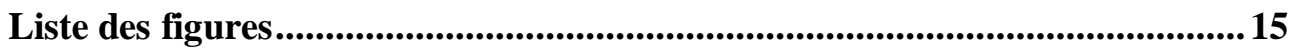

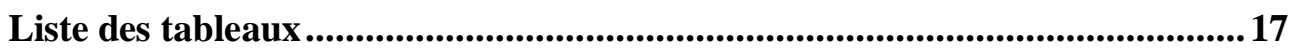

Chapitre 1 Introduction et objetifs de la recherche........................................19

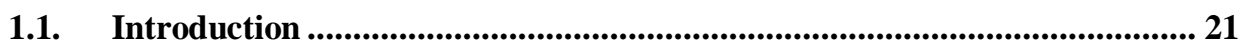

1.2. Objectifs de la thèse .............................................................................. 24

1.3. Organisation du travail....................................................................... 25

Chapitre 2 L'état de l'art sur l'innovation, système d'innovation et politique d'innovation ...............................................................28

2.1. État des connaissances sur l'innovation ........................................................30

2.1.1. La définition de l'innovation ............................... 30

2.1.2. Les types d'innovation .................................. 32

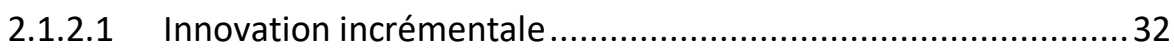

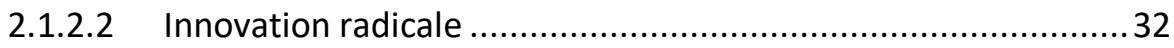

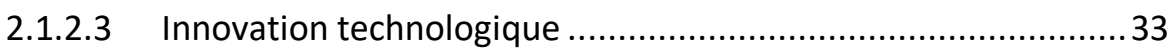

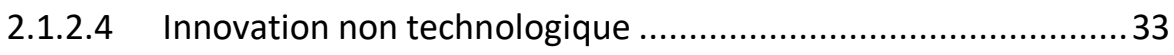

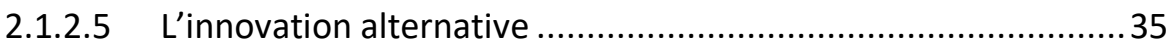

2.1.3. Les modéles de génération de l'innovation.............. 36

2.1.3.1 La première génération ou recherche-poussée «Research push» .

2.1.3.2 La deuxième génération ou à demande «Demand pull» ............37

2.1.3.3 La troisième génération ou du couplage «Coupling» ..................38 
2.1.3.4 Le modèle de la quatrième génération ou collaboratif «Collaborative»

2.1.3.5 La cinquième génération ou stratégique et intégrée "Strategic and integrated»

2.1.4. Les modèles de gestion d'innovation...................... 42

2.1.4.1 Le modèle de gestion totale de l'innovation «totale innovation management» (TIM).

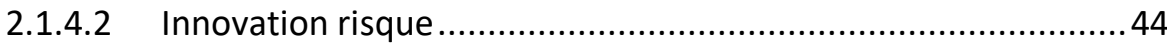

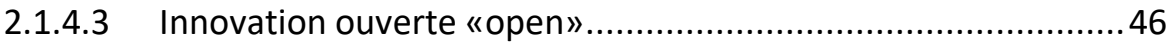

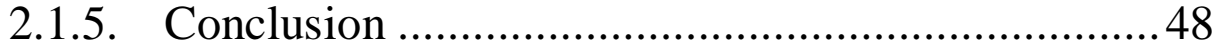

2.2. Ètat de l'art sur le Système d'innovation .................................................... 49

2.2.1. Le concept du système national d'innovation : origine, dimension et caractéristiques............................4 49

2.2.1.1 Origine de l'approche système national d'innovation...............50

2.2.1.2 Définition du système national d'innovation ...........................51

2.2.1.3 Le réseau du système national d'innovation............................5 52

2.2.1.4 Caractéristiques du système national d'innovation ..................53

2.2.2. Institution et système d'innovation .......................... 54

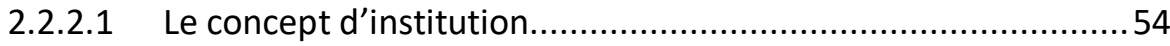

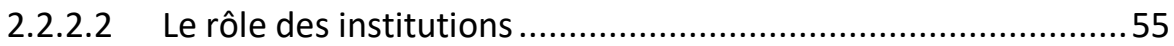

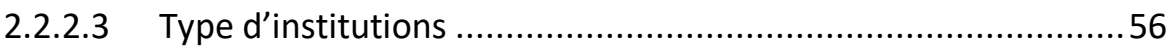

2.2.2.4 La fonction des institutions ................................................... 57

2.2.3. La fonction et organisation du système national

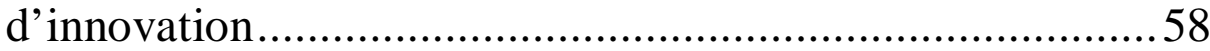

2.2.3.1 Organisations, institution et système national d'innovation .....58

2.2.3.2 La fonction du système national d'innovation ..........................59 
2.2.3.3 Caractéristique des liens entre les différents acteurs 60

2.2.4. Les blocs de construction et infrastructure économique du système national d'innovation .....................61

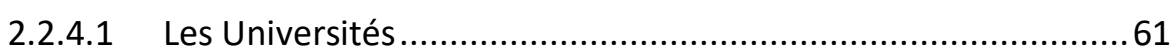

Le secteur public ......................................................62

2.2.4.2 62

2.2.4.3 Le secteur d'activité ........................................................62 62

2.2.4.4 Les nouveaux composants d'infrastructures........................62

2.2.4.5 Le bloc de développement politique .................................63

2.2.5. Infrastructure économique et systéme national

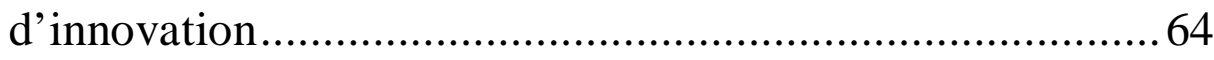

2.2.5.1 Le concept infrastructure économique..............................64

2.2.5.2 Caractéristiques de l'infrastructure économique...................65

2.2.5.3 L'infrastructure de la connaissance ...................................65

2.2.5.4 Le rôle de l'infrastructure de la connaissance .........................66

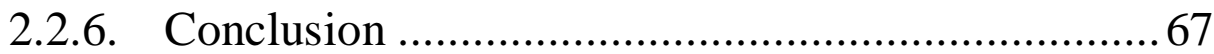

2.3. Les types du système d'innovation .................................................. 67

2.3.1. Le système sectoriel d'innovation..........................67

2.3.1.1 La typologie du système sectoriel d'innovation .....................69

2.3.1.2 Le concept du régime technologique................................ 70

2.3.2. Le système technologique d'innovation.................. 73

2.3.2.1 La définition du système technologique ..................................73

2.3.2.2 Les éléments du système technologique ............................. 74

2.3.2.3 La discontinuité technologique et la dynamique industrielle ....74

2.3.3. Le système régional d'innovation ............................ 75 
2.3.3.1 Définition du systè,e régional d'innovation .75

2.3.3.2 Les formes institutionnelles du système régional d'innovation. 76 2.3.4. Conclusion ................................................... 77

2.4. État de l'art sur la politique d'innovation ....................................................... 78

2.4.1. État des connaissances sur la politique d'innovation ...............................................78

2.4.1.1 Définition de la politique...................................................... 78

2.4.1.2 Politique d'innovation ......................................................... 79

2.4.2. Les différents types de la politique d'innovation ....81

2.4.2.1 Les politiques axées sur la mission ......................................... 81

2.4.2.2 Les politiques axées sur l'invention ......................................... 82

2.4.2.3 Les politiques axées sur le système ......................................... 82

2.4.3. Les approches de la politique d'innovation.............82

2.4.3.1 L'approche de la politique d'innovation fondée sur l'échec du marché

2.4.3.2 L'approche de la politique d'innovation fondée sur le système d'innovation

2.4.3.3 Innovation, dépendance à l'égard du cheminement et politiques 83

2.4.4. Instrument politique ....................................... 84

2.4.5. Evaluation de la politique d'innovation .................84

2.4.6. Les modes de la politique d'innovation et système

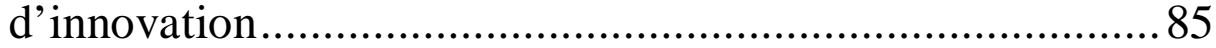

2.4.6.1 Le modèle du système transitionnel de Galli et Teubal .............85

2.4.6.2 Le modèle vertical d'innovation de Andersen et Lundvall.........89

2.4.6.3 Le modèle Franz Tödtling et Michaela Trippl de la politique d'innovation régionale. 90 
2.4.6.4 Le modèle de De Lucio, Mas-Verdu et Tortosa des générations de l'innovation

2.4.6.5 Le modèle de la danse de la politique d'innovation de Kuhlmann, Shapira, et Smits (IPT)

2.4.6.6 Les modèles des instruments dans la politique d'innovation de Borrás et Edquist

2.4.6.7 Le modèle de la mobilisation de la politique de Peck et Theodore

Conclusion

2.4.6.8 Conclusion ............................................................. 97

2.4.7. L'industrie pharmaceutique en Algérie et politique 98

2.4.7.1 L'industrie pharmaceutique en Algérie...............................98

2.4.7.2 La politique pharmaceutique en Algérie.......................... 102

2.4.7.3 L'évolution de la politique d'innovation du secteur pharmaceutique en Algérie......................................................... 104

2.4.7.4 Conclusion ............................................................ 110

Chapitre 3 Cadre conceptuel de la recherche......................................... 111

3.1. Proposition du modèle et élaboration d'hypothèses ...................................113

3.2. Développement d'hypothèses .....................................................................114

3.2.1. Activités d'innovation ........................................ 117

3.2.2. Les acteurs d'innovation.................................... 122

3.2.3. Les instruments politiques publics de l'innovation 125

3.2.4. Les résultats d'innovation.................................... 129

3.2.5. Obstacles et freins à l'innovation .......................... 131

3.3. Conclusion.....................................................................................131

Chapitre 4 Méthodologie de Recherche ..................................................... 133

4.1. Le design de la Recherche...........................................................................135

4.2. La démarche de la collecte des données....................................................137 


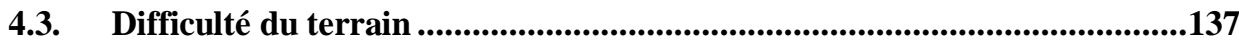

4.4. Sources de données ............................................................................138

4.5. Elaboration et choix du questionnaire........................................................139

4.6. Les techniques Statistiques pour l'Analyse des Données..............................140

4.6.1. La Modélisation par Équation Structurelle :’’ L’Approche PLS', (Partial Least Squares)..................... 140

4.6.2. Les critères de validation du modèle.................... 141

4.6.3. Evaluation du modèle structurel ......................... 142

4.6.4. Le software du PLS ........................................ 143

Chapitre 5 Étude empirique sur le terrain .................................................. 145

5.1. Analyse descriptive ......................................................................................147

5.1.1. Information générale .................................... 147

5.1.2. Les résultats (outputs) de l'innovation .................. 150

5.1.3. L’activité de l'innovation des entreprises ............ 151

5.1.4. Les obstacles à l'innovation................................ 153

5.1.5. Les obstacles à l'adoption de la technologie ......... 154

5.1.6. Conclusion ................................................... 154

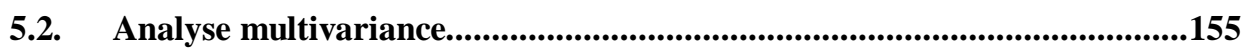

5.2.1. Fiabilité et validité des mesures .......................... 155

5.2.2. Essais des hypothèses ...................................... 157

5.2.3. Modèle structurel........................................... 158

5.2.4. Conclusion .................................................... 161

Chapitre 6 Conclusions générales ...................................................................... 163

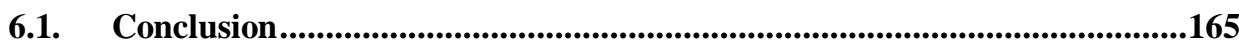

6.2. Implication et recommandation pour la politique d'innovation dans les pays en développement ..........................................................................................................169

6.3. Etendue et limitation de l'étude ................................................................170 
Table des matières

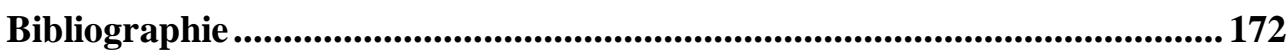

6.4. Bibliographie....................................................................................................174

Annexe 209

7.1 Annexe 1 Le guide de l'interview 210 
Evaluation de la politique d'innovation sur un échantillon d'entreprises algériennes 


\section{Liste des figures}

Figure 1.1 Organisation du travail 26

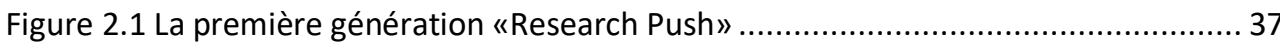

Figure 2.2 La deuxième génération ou" Demand Pull" ................................................ 38

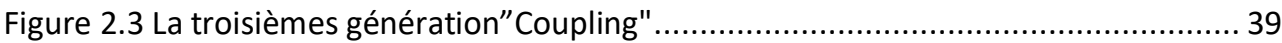

Figure 2.4 La quatrième génération «Collaborative" .............................................. 40

Figure 2.5 La cinquièmes génération"Strategic and Integrated"................................... 41

Figure 2.6 Les éléments de la gestion totale d'innovation........................................... 44

Figure 2.7: Le modèle de l'innovation risque ........................................................... 45

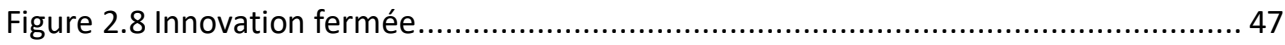

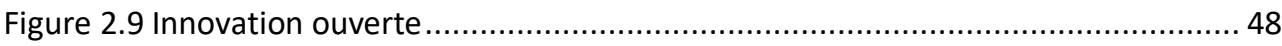

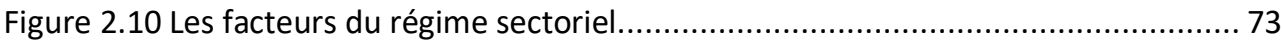

Figure 2.11 La dynamique industrielle et la discontinuité technologique ....................... 75

Figure 2.12 La première phase du système d'innovation ........................................... 86

Figure 2.13 La deuxième phase du système d'innovation ........................................... 87

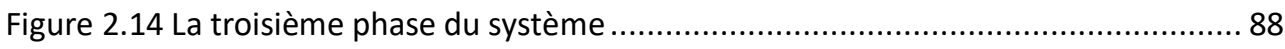

Figure 2.15 Le system de production simple en relation avec les taches ....................... 90

Figure 2.16 Les contraintes à l'innovation par région.............................................. 91

Figure 2.17 Le modèle de génération du système d'innovation ................................ 92

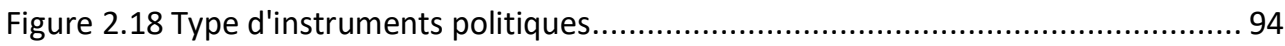

Figure 2.19 Principaux types d'instruments politiques............................................... 95

Figure 2.20 Les modes de la politique d'innovation et système d'innovation ................... 97

Figure 2.21 Evolution des importations et des exportations en Algérie par année............ 99

Figure 2.22 L'évolution du produit intérieur brut (PIB) en Algérie ................................. 99

Figure 2.23 L'évolution de la population Algérienne par année.................................... 100

Figure 2.24 L'évolution des importations des médicaments par année par unité en Millions de DA

Figure 2.25 La part des pays étrangers des importations des médicaments en Algérie par unité

Figure 2.26 Le nombre d'entreprises pharmaceutiques par activité ........................... 103

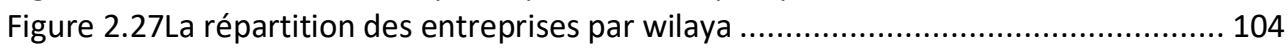

Figure 2.28 L'évolution de la politique de l'industrie pharmaceutique en Algérie........... 109

Figure 2.29 La mise sur le marché et l'enregistrement des médicaments..................... 109

Figure 3.1 Modèle théorique (basé sur divers auteurs cités) ..................................... 114

Figure 3.2 Le modèl théorique proposé................................................................ 117

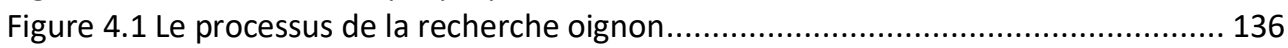

Figure 5.1 Répartition des répondants par activité ................................................. 147

Figure 5.2 Répartition des répondants par région.................................................. 148

Figure 5.3 La position des répondants........................................................... 149 
Evaluation de la politique d'innovation sur un échantillon d'entreprises algériennes

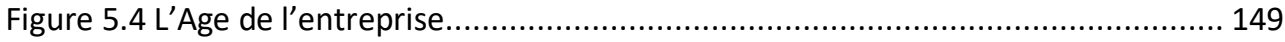

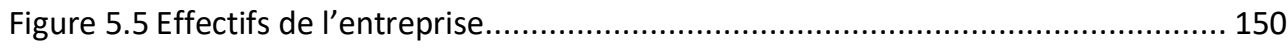

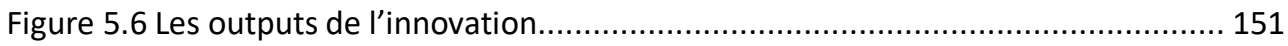

Figure 5.7 L'activité d'innovation des entreprises pharmaceutiques ........................... 152

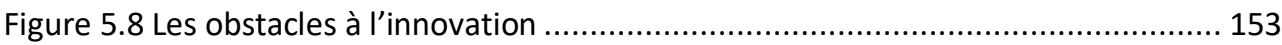

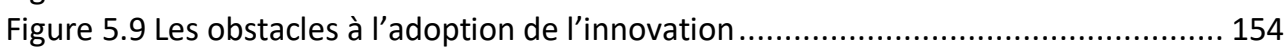

Figure 5.10 Modèle d'équations structurelles estimées à l'aide de l'échantillon............. 159 


\section{Liste des tableaux}

Tableau 2.1 L'activité et caractéristique des phases de transitions 89

Tableau 4.1 Les critères de validation du modèle PLS 135

Tableau 4.2 Les critères de validation du modèle PLS. Error! Bookmark not defined.

Tableau 5.1 Mesures de fiabilité 157

Tableau 5.2 Mesures de fiabilité .... Matrice de corrélation entre les variables latentes (Fornell\&Lacker,1981) 157

Tableau 5.3 Effets directs, variances expliquées et test de Stone Geiser Q2 pour des variables endogènes. 


\section{Liste des annexes}

Annexe 1 Le guide de l'interview 


\section{Chapitre 1 Introduction et objetifs de la recherche}


Evaluation de la politique d'innovation sur un échantillon d'entreprises algériennes 


\subsection{Introduction}

Le choix des politiques d'innovation est une décision importante pour les pays en développement. Il y a une demande claire pour une politique d'innovation qui dépend des conditions du pays. Cela a suscité l'intérêt des chercheurs lesquels relient l'innovation et la politique d'innovation au développement (A. D. Andersen, 2012b, 2012a; Capriati, 2017; Dantas \& Bell, 2011; Dutrénit \& Sutz, 2014, p. 388; Iizuka \& Katz, 2012; Katz, 2015; Morris, Kaplinsky, \& Kaplan, 2012; Sesay, Yulin, \& Wang, 2018)

Les politiques publiques sur l'innovation sont un vaste sujet qui a été utilisé comme un outil par l'administration publique (Amdaoud, 2017; Borrás \& Laatsit, 2019; Kuhlmann \& Ordóñez-Matamoros, 2017; Lerner \& Stern, 2019). En outre, les politiques d'innovation scientifique et technologique peuvent contribuer à améliorer le comportement des agents économiques, à articuler l'offre et la demande de connaissances, à stimuler le développement des secteurs et à promouvoir de nouveaux domaines de compétitivité(Kuhlmann \& Ordóñez-Matamoros, 2017, p. 13).

Cependant, les politiques d'innovation sont interprétées comme un phénomène complexe (Ocde, 2005) qui est souvent lié aux systèmes d'innovation. Lundvall a défini les systèmes d'innovation (1992) comme " un système ouvert, évolutif et complexe englobant des institutions et des structures économiques " et, par conséquent, la qualité de ses éléments et les relations entre eux détermineront la dynamique et l'orientation de l'innovation.

Dans la mesure où la politique d'innovation est èlaborèe à partir des interactions entre acteurs et à partir de l'apprentissage, l'émergence d'un partenariat entre le Sud et le Nord ou entre les régions développées et sous-développées peut faciliter les échanges de connaissances et d'expériences et ainsi favoriser une politique contingente. En termes de connaissances, le monde académique met davantage l'accent sur l'émergence de l'innovation dans tous les pays à travers des organisations internationales telles que les réseaux GlobeLics et AfricaLics, le réseau Triple Helix, et le forum Eu-SPRI

L'objectif principal du réseau GlobeLics est de stimuler la recherche sur l'innovation chez les universitaires ; AfricaLics s'en est inspiré. Le réseau AfricaLics met en relation des chercheurs travaillant sur le développement de l'innovation pour les pays africains. De plus, le réseau Triple hélice examine la relation entre le milieu universitaire, le gouvernement et l'industrie. Le Forum d'études politiques européennes sur la recherche et l'innovation (Forum Eu-SPRI) permet en outre aux chercheurs de se concentrer sur les dimensions interdisciplinaires de la politique et de la gouvernance dans le domaine de la créativité et de l'innovation.

D'autre part, les décideurs politiques s'intéressent à la façon d'importer des connaissances par le biais de la collaboration entre les nations. A cet égard, les pays émergents sont les plus touchés par l'expérience des pays développès, avec des instruments tels 
que les programmes de coopération institutionnelle entre les administrations publiques des Etats membres de l'UE et d'autres pays en développement.

Ces initiatives visent également à comprendre et à analyser la manière dont la politique d'innovation devrait être conçue sur la base d'échanges d'expériences entre pays développés et pays en développement. Malgré cela, la politique d'innovation reste basée sur des concepts généraux et macroéconomiques (Djeflat, Devalan, \& Youcef Ettoumi, 2007). Ainsi, les pays en développement ont tendance à échouer dans leurs politiques d'innovation malgré les efforts déployés pour les développer (Kuhlmann \& OrdóñezMatamoros, 2017).

La question de savoir quand et comment élaborer une politique d'innovation pourrait être abordée de diverses manières. Pour cette raison, le choix de la politique d'innovation reste une question controversée (Kuhlmann \& Ordóñez-Matamoros, 2017). ). Des questions telles que la mesure de l'innovation (par exemple, le manuel de Frascati et d'Oslo) ou la relation entre les acteurs, la production et le transfert de connaissances, ou encore les théories du système d'innovation ou de la triple hélice (Leydesdorff, 2000) attirent l'attention des chercheurs.

Dans de nombreux pays, la politique d'innovation est encore caractérisée par des concepts généraux et macroéconomiques. Chaque édition du Manuel d'Oslo (Collecting \& Data, 2005; Ocde, 2000; Publishing, 2006) s'efforce de renforcer les différentes méthodes pour rendre l'innovation significative dans les pays. Par exemple, la série des manuels d'Oslo a introduit plusieurs versions.

L'amélioration de l'application des modèles d'innovation des pays les plus avancés a été amorcée par un élargissement du partenariat africain, par exemple. Cette initiative est connue sous le nom de " Perspectives de l'innovation en Afrique II " (NEPAD, 2014) ; elle est le résultat de la réunion du Conseil africain pour la science et la technologie (ACST). Ce dit Conseil a adopté un plan d'action pour la croissance et l'amélioration de la vie des peuples africains. Ses membres ont souligné la nécessité d'élaborer des indicateurs de la STI en Afrique. Une première version, publiée en 2010, a rassemblé les résultats de 19 États membres. Une deuxième version qui est arrivée en 2014 pour compléter la première a rassemblé les 35 États membres. Ce plan d'action a permis de rassembler les indicateurs et les statistiques nationaux sur la STI pour l'élaboration des politiques. Toutefois, cette initiative est théorique.

D'un point de vue académique, Edler et Faberge (2017) distinguent trois types de politique d'innovation : premièrement, une politique d'innovation orientée vers la mission globale (Charles Edquist \& Zabala-Iturriagagoitia, 2012) ; deuxièmement, une politique d'innovation résultant de la recherche et du développement (V Bush, 1945) et enfin, une politique axée sur le système d'innovation.

Ce dernier point de vue, orientation de la politique d'innovation par le biais du système national d'innovation, a été largement assumé par les chercheurs universitaires (Barre, 
1996; Freeman, 1987; R. Nelson, 1993). En même temps, d'autres théories ont été développées pour approfondir la question de l'innovation en tenant compte du modèle prédominant d'innovation dans la région (de Lucio, Mas-Verdu, \& Tortosa, 2010). Chaque modèle ou approche a été examiné sur la base d'une caractéristique spécifique d'un pays ou d'un contexte particulier. De plus, d'autres modèles ont émergé du concept de système national d'innovation, comme le régional et même le local (Bergman, Maier, \& Tödtling, 1991; Philip Cooke, Gomez U, Etxebarria, \& U, 1997)et les travaux de Breschi et Malerba (1997) qui combinent la dimension nationale, sectorielle et technologique...

En ce qui concerne l'évaluation de la politique d'innovation, Magro et Wilson (2013) considèrent que non seulement les décideurs politiques devraient décider des instruments de cette politique, mais que dans la conception de la politique d'innovation, tous les acteurs et agents impliqués devraient contribuer.

Dans ce même ordre d'idée, Herstatt et al (2008) suggèrent que le système national d'innovation devrait être bien établi et offrir de nouveaux cadres conceptuels qui expliquent mieux le fonctionnement de l'innovation. Par conséquent, la question de l'étude de l'échec ou du succès du système d'innovation s'est révélée indispensable. À cet effet les mesures politiques visent à réduire les obstacles à l'innovation au sein des entreprises quelle que soit la taille de l'entreprise et doit être d'ériger au niveau micro afin de diminuer le moins possible des obstacles à l'activité de l'innovation (D'Este, Iammarino, Savona, \& von Tunzelmann, 2012). Cela fonctionne quand les agents qui participent aux processus d'innovation sont impliqué dans l'orientation politique (Amdaoud, 2017).

Si l'on considère le cas de l'Afrique, et surtout du Maghreb qui fait l'objet de cette thèse, selon le classement du " Global Innovation Index (GII), 2017 1" de 127 pays pour la période de 2011 à 2017, un résultat et un classement médiocre est observé pour l'Algérie. Sur 127 pays en 2017 : Tunisie est $74^{\text {ème }}$, Maroc $72^{\text {ème }}$, Egypte $105^{\text {ème }}$, Algérie $108^{\text {ème }}$. Cela la place au dernier rang des pays du Maghreb et loin des pays asiatiques comme l'Inde (66 ${ }^{\text {ème }}$ en 2017) ou les pays d'Amérique latine.

Les travaux de Djeflat (Djeflat, 2003, 2004, 2008, 2010; Djeflat et al., 2007) sur la politique d'innovation en Algérie, proclament que l'approche de l'innovation en Algérie a toujours été basée sur l'offre plutôt que sur le renforcement de la demande d'innovation. D'autre part, Amdaoud (2017) dans son analyse du système national d'innovation

\footnotetext{
${ }^{1}$ L'indice mondial de l'innovation (Global Innovation Index - GII) fournit des statistiques détaillées sur la performance de 126 pays représentant $90,8 \%$ de la population mondiale et $96,3 \%$ du PIB mondial. Ses 80 indicateurs explorent une vision large de l'innovation, y compris l'environnement politique, l'éducation, les infrastructures et la sophistication des entreprises.
} 
en Algérie, suggère que l'identification des potentialités locales est fondamentale pour la formation des capacités technologiques.

Le travail de terrain présenté dans cette thèse concerne le secteur pharmaceutique. La raison réside dans sa croissance rapide en peu de temps (Djeflat \& Hamadi, 2016). Après l'indépendance de l'Algérie en 1962, la forte croissance démographique a entraîné une augmentation de la demande de médicaments qui a ensuite affecté le taux des importations de médicaments. Une politique d'ouverture du marché a donc été adoptée en 1992. En 2002, une politique a été introduite pour promouvoir les médicaments génériques et la production nationale. En 2015, le Premier ministre de l'époque, Monsieur Abdelmalek Salel, a déclaré dans la presse (djazairess, 2015) la promotion de la production locale comme politique de substitution à l'importation de médicaments. Ainsi, l'adaptation des entreprises pharmaceutiques à ce nouveau scénario a ouvert des perspectives de développement dans le domaine de l'innovation, en mettant l'accent sur toutes les politiques appropriées adaptées à ces entreprises. A cet effet, les études préalables demontre les obstacles de l'innovation malgrés la focalisation politiques vis-àvis le secteurs pharmaceutique (Casadella \& Younes Bouacida, 2020; Nasreddine, 2017)

\subsection{Objectifs de la thèse}

L'intérêt de cette thèse est de comprendre la politique d'innovation dans les pays en développement et d'étudier les facteurs clés qui déterminent l'innovation dans les entreprises, en examinant les actions de l'administration et les réactions des agents économiques impliqués.

Donc l'objectif global de cette thèse est d'une part, de faire progresser les connaissances scientifiques sur l'adaptation de la politique d'innovation aux besoins d'innovation des entreprises sur la base d'un modèle conceptuel qui inclut le système d'innovation et la politique d'innovation.

Et également pour évaluer, à partir des avis des agents économiques, si les politiques appliquées jusqu'à présent ont été efficaces et de savoir le cas échéant quelles seraient leures propositions.

D'autre part, l'objectif est de comprendre la pertinence de la politique d'innovation dans le cas de l'Algérie à partir du travail de terrain effectué dans les entreprises pharmaceutiques algériennes.

La question essentielle abordée dans ce travail de recherche et à laquelle nous allons essayer d'apporter notre contribution, est la suivante :

- Quelle est la politique d'innovation appropriée pour le développement des entreprises pharmaceutiques en Algérie?

Nous essayons de répondre également à d'autres questions secondaires telles que : 
Chapitre 1 Introduction et objetifs de la recherche

- Comment comprendre les aspects constructifs d'une politique d'innovation efficace ?

- Comment renforcer cette politique d'innovation en impliquant tous les acteurs du système d'innovation?

\subsection{Organisation du travail}

Ce mémoire de thèse est organisé comme suit (figure 1.1) :

Le chapitre premier concerne, l'introduction générale et les objectifs de cette thèse.

Le deuxième chapitre présente l'état de l'art sur l'innovation, le système d'innovation et la politique d'innovation. Il est divisé en quatre sections. La première section concerne les types d'innovation ; elle permet de mieux comprendre l'activité d'innovation dans les entreprises ainsi que la demande. Dans la section II, le concept du système national d'innovation est ensuite introduit afin de comprendre le comportement des acteurs dans le système ainsi que l'importance des instituts et les infrastructures. Cette dernière section nous amène à la nécessité de comprendre les types du système d'innovation que nous introduisons dans la section III. La section IV est consacrée à la politique d'innovation et ces modes. La fin de cette section sera réservée à l'analyse du secteur pharmaceutique en Algérie ; elle représente la dernière section du chapitre.

Le troisième chapitre sera consacré à sélectionner, d’abord dans la première section, les modèles les plus pertinents à notre cas sur le système d'innovation et la politique d'innovation. La deuxième section de ce chapitre est consacrée à la présentation les hypothéses de notre recherche et cela à partir de la littérature. Cette conceptualisation sera une proposition à notre modèle théorique présenté dans cette thèse.

Le quatrième chapitre présentera la partie empérique de l'étude. Elle consiste à définir la méthodologie que nous avons choisie. Nous y présentons le guide de l'entretien et la stratégie suivie afin de collecter l'information. Nous introduisons, dans ce chapitre, de façon sommaire les méthodes d'analyse qui sont utilisées pour répondre aux objectifs de cette thèse.

Le cinquième chapitre est rèpartie en deux sections. La première se rapporte à la partie descriptive de l'etude et la deuxième est consacrée à l'analyse des multivariances.

Le dernier chapitre est consacré à la conclusion de ce travail. 


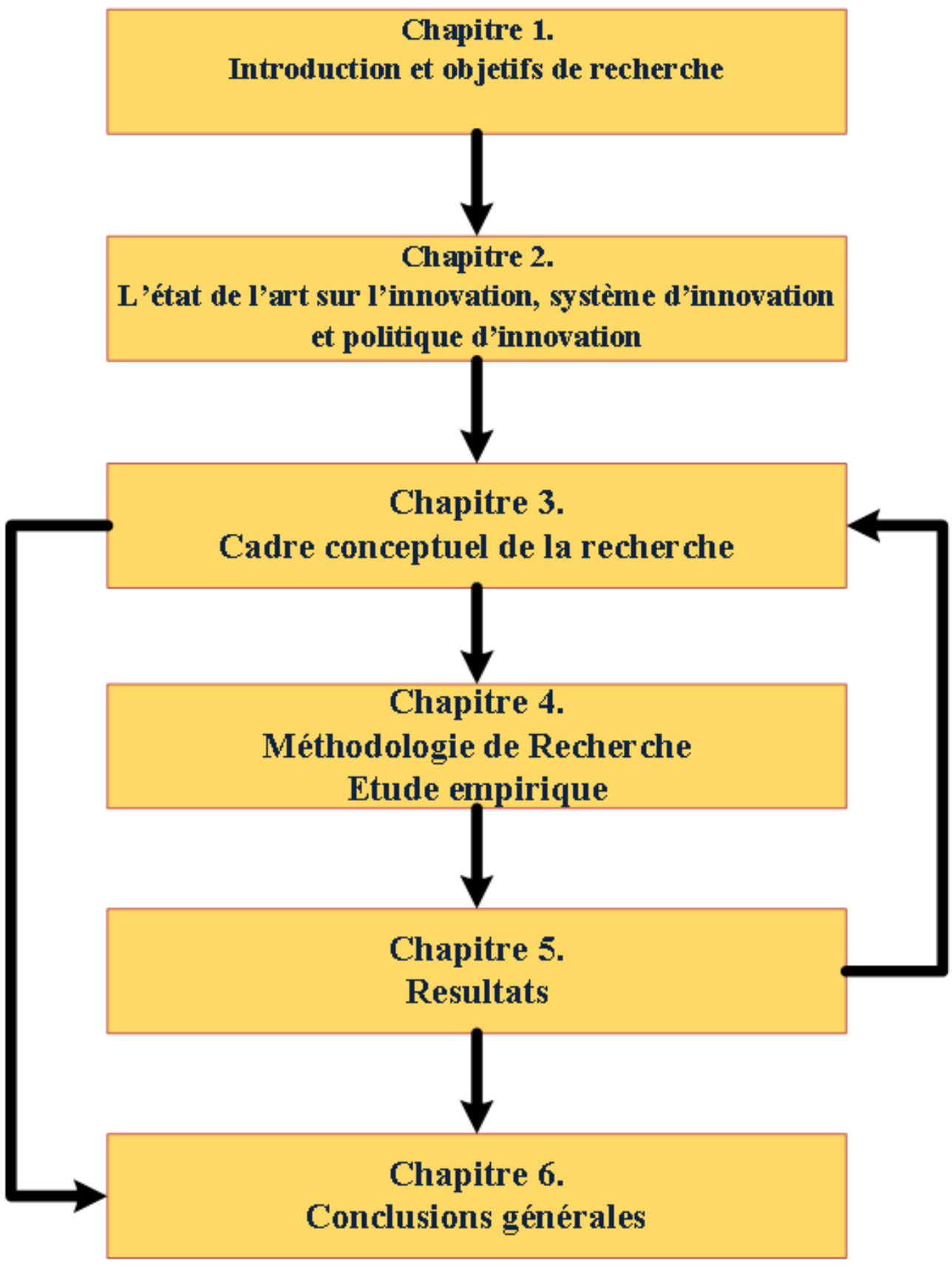

Figure 1.1 Organisation du travail 


\section{Chapitre 2 L'état de l'art sur l'innovation, système d'innovation et politique d'innovation}


Chapitre 2 L'état de l'art sur l'innovation, système d'innovation et politique d'innovation 
L'objectif de ce chapitre est de comprendre les concepts principaux qui amènent à notre modèle théorique. Car la politique d'innovation est perçue au niveau macroéconomique et notre but est de comprendre l'impact de cette politique au niveau microéconomique. A cet effet nous allons définir dans la première section les aspects théoriques qui existent au niveau microéconomique pour démontrer les facteurs qui déterminent l'innovation dans les entreprises. Nous introduisons ensuite dans la section (2) le système national d'innovation afin de comprendre le comportement des acteurs au sein d'un système et les relations qui peuvent $\mathrm{y}$ exister. La troisième section a pour but d'expliquer le rôle de la politique d'innovation au sein du système. Finalement nous procédons à l'établissement d'un modèle théorique permettant de montrer le processus du système politique d'innovation.

\section{1. État des connaissances sur l'innovation}

\subsubsection{La définition de l'innovation}

Le concept d'innovation dans une entreprises est évoqué par Joseph Alois Schumpeter dans la «théorie de l'évolution économique» (1934). Il suggère que l'esprit entrepreneurial est la clef de succès au sein d'une entreprise, et l'entrepreneur créateur et dynamique est la source de progrès dans une entreprise.

Dès lors Schumpeter (1934) énonce divers types d'innovations qui sont: l'introduction d'un nouveau produit ou une modification qualitative d'un produit existant, l'introduction d'un procédé constituant une innovation pour une industrie, l'ouverture d'un nouveau marché, le développement de sources nouvelles d'approvisionnement en matières premières ou en d'autres inputs, les évolutions de l'organisation industrielle (Ocde, 2005, p. 18).

Par ailleurs, Taylor (1914) introduit la vision de l'amélioration de procédé par le biais de l'organisation du travail afin d'assurer l'efficacité et l'efficience dans une entreprise.

De là, l'innovation est un processus complexe qui est définie dans le manuelle d'Oslo (2005, p. 7) comme l'interaction entre les possibilités offertes par le marché et la base des connaissances et les aptitudes de la firme. Car ces interactions sont les interlocuteurs par plusieurs acteurs au sein du système qui contribue à l'innovation.

En l'occurrence, la vision de l'innovation a pris plusieurs sens en matière de typologie et développement :

- Schmidt et Rammer (2007) font la diffèrence entre la production de l'innovation et le processus d'innovation dans lequel ils distinguent innovation technologique et non technologique.

- Freeman (1987) a fait la distinction entre l'innovation radicale et l'innovation incrémentale dans laquelle une technologie ou le degré de nouveauté d'une innovation a changé. 
- Selon Tushman et Anderson la façon de faire les choses pour l'innovation est continue ou discontinue (1986).

- Christensen (1997) parle de durable ou perturbatrice, la difficulté à rompre avec les technologies et les manières d'innover. Car les gestionnaires peuvent avoir besoin d'explorer de nouvelles façons de faire les choses qui sont destructrices des succès existants.

- Le cycle de vie de l'innovation dont parlent, Abernathy et Utterback (1981), explique comment l'innovation se rapporte à l'émergence précoce, à une période de fermentation et d'incertitude, à une période de croissance et finalement à une maturité dans un marché saturé.

- L'innovation est modulaire selon Henderson et Clark (1990). Leur concept est que l'innovation n'est pas souvent incrémentale ou radicale car il existe une modification architecturale sans changer les composants du produit.

- Une innovation est ouverte ou fermée d'après Chesbrough (2003); il explique les défis de gestion de l'innovation ouverte car l'innovation implique différentes stratégies d'achat, de vente et de collaboration.

En ce qui concerne l'évaluation de l'innovation, les éditions OCDE (2005, p. 15) ont consacré beaucoup de manuels destinés à expliquer une méthodologie de mesure de l'innovation.

- Méthode type proposée pour les enquêtes sur la recherche et le développement expérimental - Manuel de Frascati - Cinquième édition (OCDE: 1993).

- Définitions et conventions de base pour la mesure de la recherche et du développement expérimental (R-D) Résumé du Manuel de Frascati (OCDE: GD1993).

- Méthode type proposée pour le recueil et l'interprétation des données sur la balance des paiements technologiques - Manuel BPT (OCDE: GD1992).

- Principes directeurs proposés par l'OCDE pour le recueil et l'interprétation des données sur l'innovation technologique - Manuel d'Oslo - deuxième édition (OCDE/CE/Eurostat: 1996).

- Les données sur les brevets d'invention et leur utilisation comme indicateurs de la science et de la technologie - Manuel Brevet (OCDE/GD(1994).

- La mesure des ressources humaines consacrées à la S-T - Manuel de Canberra (OCDE/CE/Eurostat: GD1995). 


\subsubsection{Les types d'innovation}

A travers la définition de l'innovation nous apercevons qu'il y a différentes formes d'innovation, tout dépend de la valeur ajoutée que l'on prétend avoir comme résultat et avec la condition qu'elle soit unique et dífférente. Cette différenciation de l'innovation nous a permis de nous projecter par la suite sur les types d'innovation qui s'apparentent aux caractéristiques de l’innovation dans les pays en dévelopement ou émergents.

\subsubsection{Innovation incrémentale}

L'innovation incrémentale est la modification ou l'amélioration dans le produit d'origine (Roy Rothwell \& Gardiner, 1988). Elle est la forme la plus courante car elle permet à l'entreprise d'exploiter les connaissances et les savoirs acquis à travers l'expérience.

L'innovation incrémentale dans le processus est la modification dans l'activité quotidienne de l'entreprise. Cette forme de modification permet de faciliter la manière de produire et a un impact direct sur la production (Utterback, 1994).

Cependant, le succès d'une innovation incrémentale exige la compétence et l'ingéniosité. Les innovateurs incrémentaux adoptent une gamme de stratégies et de pratiques de gestion pour capter le rendement de leurs efforts (Dodgson, Gann, \& Salter, 2008, p. 58), notamment: chercher des avantages de coûts par rapport à la concurrence, faire des modifications mineures à la conception, créer des routines organisationnelles, des procédures et des normes pour une production plus efficace et plus économique, finalement, ajouter des fonctionnalités aux produits existants.

\subsubsection{Innovation radicale}

L'innovation radicale est l'émergence d'une nouvelle conception qui configure le cheminement productif d'un produit, quant à la façon établie à la configuration ou la conception du produit (Afuah \& Utterback, 1997; Utterback, 1994); car l'innovation radicale est caractérisée par la rupture avec le processus de production du passé (Rosenberg, 1982).

En l'occurrence, Tushman et Anderson (1986) ont analysé les périodes d'élaboration d'une innovation radicale dans laquelle ils supposent que le changement technique se produit lentement et cumulativement jusqu'à ce qu'il soit marqué par une avance importante. Car ce phénomène se produit dans un intervalle de temps de trente ans.

En revanche, les innovations radicales sont des événements critiques qui remodèlent les conceptions, les connaissances et la nature de la concurrence sur le marché des produits (Dosi, 1982; Utterback, 1994). Car elles perturbent la position des entreprises sur le marché et donnent accès à l'intégration de nouvelles entreprises lesquelles dépassent les entreprises en place, ce qui permet de transférer les connaissances et les expériences d'une industrie à l'autre (Christensen, 1997). 
A cet effet Dodgson et al. (2008, p. 59) suggèrent des stratégies de pratique afin que les entreprises se préparent aux innovations radicales: Volonté d'être ouvert à de nouvelles idées de l'extérieur de l'entreprise et du secteur, recherche et analyse en continu de l'environnement technologique et du marché, prendre des options sur différents futurs en investissant dans un portefeuille de technologies, création de nouveaux liens et relations au sein des systèmes d'innovation, réorganiser les structures organisationnelles pour s'assurer que certaines parties participent à des travaux exploratoires, obtenir de nouvelles capacités par l'acquisition ou l'embauche, investir dans de nouveaux domaines technologiques quelque peu éloignés de la pratique actuelle, construire des réseaux et des alliances de l'extérieur de l'industrie de base, être conscient des nouveaux comportements des clients et refuser les vieilles habitudes ou les façons de faire les choses en cas de besoin immédiat (Dodgson et al., 2008, p. 59).

\subsubsection{Innovation technologique}

L'innovation technologique, selon le manuel d'Oslo (2005, p. 9), est définie comme l'établissement ou la commercialisation d'un produit dans l'objectif d'offrir un meilleur service ; et non seulement dans le produit, car il existe aussi une technologie des procédés qui à travers laquelle l'entreprise adopte une nouvelle méthode de production ou une méthode de distribution nouvelle. Cette adoption ce concrétise en matière de nouveaux matériaux, une nouvelle ressource humaine ou une nouvelle méthode de travail.

En l'occurrence, Dodgson et al. (2008, p. 66) mettent l'accent sur la différence dans l'utilisation. L'adaptation et la durée d'adoption de la technologie diffèrent d'un secteur d'activité à un autre. Ainsi ils évoquent l'utilisation des technologies d'informations et de communication. Ils considèrent qu'ils sont présents dans le processus de l'innovation technologique.

\subsubsection{Innovation non technologique}

D’après Schumpeter (1934), l'innovation non technologique est considérée comme l'introduction d'un nouveau produit ou l'introduction d'un procédé.

A cet effet, l'innovation non technologique selon L'OCDE (Ocde, 2005, p. 97) englobe toutes les activités d'innovation qui ne relèvent pas de l'innovation technologique. Car, c'est une innovation purement organisationnelle et administrative.

Dès lors, ce type d'innovation est associé aux incertitudes qui impliquent un investissement dans l'innovation, les couts, la réalisation, la création, l'utilisation des nouvelles connaissances et efficiences (Knight, 1921).

En l'occurrence, Damanpour (1991) explique l'innovation non technologique ou organisationnelle comme diffusion et adoption de l'innovation au sein des entreprises. 
Par ailleurs, l'innovation non technologique consiste à l'adoption de techniques avancées de gestion, la modification importante des structures organisationnelles et l'adoption d'orientations stratégiques nouvelles ou leur modification (Ocde, 2005, p. 97).

\subsection{Innovation inversèe «Reverse innovation»}

L'innovation inversée est la production dans les pays en développement, en dépit du faible niveau du pouvoir d'achat, d'une forte préoccupation par les couts de production, qui ramène à l'innovation selon les conditions locales et environnementales (Marcelle, 2017, p. 64).

En outre, les facteurs de succès de l'innovation dans les entreprises des pays en développement sont dus à l'accès et la liaison dans la connaissance locale et la vision du client (Marcelle, 2017, p. 64). Car l'approche des firmes des pays en développement est censée s'adapter avec le pouvoir d'achat. Cette approche est considérée comme une opportunité à utiliser l'innovation locale qui s'adapte à l'environnement local.

Immelt et al. (2009) ont inventé le terme «innovation inversée». Ils ont étudié comment les grandes multinationales occidentales développent des idées sur les marchés émergents et diffusent des produits et services mondiaux. Ils montrent que les idées de productions commencent dans les marchés émergents puis le flux augmente vers des marchés plus établis (Govindarajan, Vijay; Trimble, 2013; Govindarajan \& Ramamurti, 2011; Immelt, Jeffrey R., Vijay Govindarajan, 2009).

En effet, Govindarajan et Trimble (Govindarajan, Vijay; Trimble, 2013) définissent l'innovation inversée comme tout type d'innovation dont l'origine est dans les pays pauvres ou en développement. Ce type d'innovation en général est adopté en premier dans les entreprises des pays en développement.

On l'occurrence, selon Govindarajan et Trimble (2013) l'apparition de cette approche est expliquée par la nécessité de présence de cinq entités: la performance, l'infrastructure, la durabilité, la régulation et les préférences. Premièrement, la performance: en raison de leurs faibles revenus, les clients des pays pauvres sont prêts à faire d'importants sacrifices en matière de performance au juste prix. Deuxièmement, l'infrastructure : les infrastructures mondiales riches sont entièrement construites; les infrastructures de l'économie émergente sont en construction. Troisièmement, la durabilité: les pays pauvres sont confrontés à de nombreux défis de durabilité les plus redoutables sur la planète. Quatrièmement, la réglementation : les systèmes de réglementation dans les économies émergentes sont moins développés; ils présentent moins de retards lorsqu'une entreprise apporte des solutions innovantes sur le marché. Finalement, les références: chaque pays a des goûts et des références distinctes (Govindarajan, Vijay; Trimble, 2013).

En revanche, le concept "Jugaad innovation” est introduit par Radjou et al. (2012). Ces derniers mettent l'accent sur les aspects non formels à travers la concentration sur la 
relation entre le client et le processus d'innovation. Par contre, Bhatti (2012) se concentre sur les moyens que les acteurs de l'innovation recherchent pour minimiser l'utilisation des ressources dans les pays en développement.

\subsubsection{L'innovation alternative}

Marcelle (2017, p. 65) propose trois approches alternatives à l'innovation dans les pays en développement: «Learning school», «The scarcity-induced framework» et «Innovation for social purpose».

Concernant l'école apprenante ou Learning school, les auteurs Bell et Figueiredo (2012) analysent les fonctions d'apprentissage dans les pays en développement ou émergents. Ils perçoivent que ces entreprises des pays en développement sont caractérisées par l'imitation de la technologie afin obtenir une production similaire. Donc cette adoption de l'innovation développe le niveau des connaissances et permet par la suite aux entreprises d'être familiarissées avec les réseaux des connaissances par la routine.

Marcelle (2004, p. 4) traite la problématique du changement rapide de la technologie dans les industries et l'adaptation avec le processus de l'innovation. Il a fait l'étude au sein de l'industrie de télécommunication africaine. Il explique l'approche de la technologie apprenante dans ces entreprises par l'accumulation des capacités nécessaires au changement technologique; d'où il introduit le concept de «Technological capability building» comme un processus d'accumulation des capacités technologique et comme une activité dont l'industrie investie.

En conséquence, l'entreprise développe et renforce les capacités à l'apprentissage à travers cinq mécanismes (Marcelle, 2004, p. 5):

- Allocation des ressources financière.

- Promouvoir une bonne organisation.

- Développer une culture organisationnelle.

- S'adapter avec les facteurs externes à travers les fournisseurs.

- L'accès aux ressources à travers le système d'innovation (Marcelle, 2004, p. 5).

A propos de «Scarcity-induced innovation» qui signifie Innovation induite par la pénurie, les chercheurs Srinivas et Sutz (2008a) démontrent que les entreprises sont confrontées à la rareté des connaissances ainsi qu'à l'incapacité financière de développement d'un certain niveau d'innovation. Ce phénomène conduit les entreprises à développer une série de compétences d'apprentissage à travers la pratique «learning by doing».

En ce qui concerne «innovation for social proposes», l'innovation à des fins sociales. Ce type d'approche est conçu à des fins sociaux dans les entreprises à but lucratifs 
comme l'apparition de certains termes «social innovation» (Grimm, Fox, Baines, \& Albertson, 2013), «inclusive innovation» (George, Mcgahan, \& Prabhu, 2012) désignant une innovation qui détermine les inégalités qui peuvent survenir dans le développement et la commercialisation des innovations, et ainsi reconnaît les inégalités qui peuvent se produire en raison de la création et de la capture de la valeur. Ainsi «Grassroots innovation» (A. Gupta, 1997; Adrian Smith, Fressoli, \& Thomas, 2014) est un mouvement qui recherche des processus d'innovation qui sont socialement inclusifs envers les communautés locales.

A travers la difinition des types d'innovation, nous considérons que certains types d'innovation ne peuvent pas exister dans les pays en développement. Cela est dû au manque de maitrise de la technologie car l'ínnovation technologique et radicale nécessite un grand niveau du savoir et savoir faire (Lennerts, Schulze, \& Tomczak, 2020). Donc, l'innovation incrémentale et non tecnologique est plus accessible au cas des entreprises dans les pays en développment (Garc, Cer, \& Comunitario, 2020). En l'occurrence, l'innovation alternative correspond bien à la situation des pays en développment puisque les entreprises dans ces pays essayent de fabriquer des produits qui resemblent au produit des pays développés mais au moindre cout vu le faible pouvoir d'achat de la population (Garc et al., 2020). En conséquence, nous allons évoquer les dífférentes générations, du modèle simple jusqu'au plus complexe pour pouvoir comprendre l'évolution et l'interlocution des différents départements au sein de l'entreprise.

\subsubsection{Les modéles de génération de l’innovation}

La génération de l'innovation est basée sur la manière dont est conçue l'innovation. Rothwell (1992) suppose que non seulement la technologie change mais également le processus d'innovation. Car d'après lui, le point de vu des entreprises envers l'innovation a changé au fil du temps. Les entreprises modifient leurs façons aux précédemment des ressources pour tirer parti des possibilités scientifiques, technologiques et du marché. A cet effet il propose cinq générations d'innovation de pensé (Dodgson et al., 2008, p. 60).

\subsubsection{La première génération ou recherche-poussée «Research push»}

Cette approche était répendue dans les années 1950 et 1960. Vannevar Bush (1945) suppose que l'innovation est un processus linéaire, commençant par la découverte scientifique, passant par l'invention, l'ingénierie et les activités de fabrication, et se terminant par la commercialisation d'un nouveau produit ou processus (Figure ci desous). Associé au conseiller en chef de la politique scientifique du gouvernement américain, Vannevar Bush (1945) a introduit l'approche R\&D dans le domaine militaire, car l'approche était un héritage de la Seconde Guerre mondiale, où le pouvoir de la science a été démontré sous la forme de la bombe nucléaire. 


\section{Processus d'Innovation de première génération}

(Poussée de la recherche)

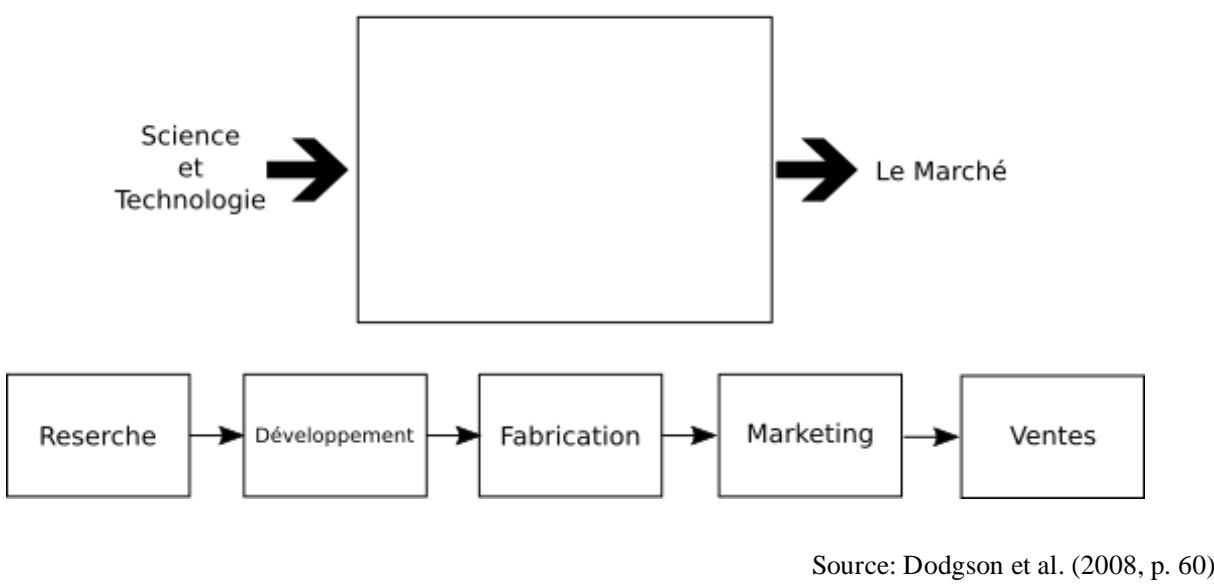

Figure 2.1 La première génération «Research Push»

\subsubsection{La deuxième génération ou à demande «Demand pull»}

Du début au milieu des années 1960, le deuxième modèle linéaire d'innovation est adopté par les décideurs publics et les gestionnaires industriels dans les économies capitalistes avancées. Dans ce modèle, les innovations découlent d'une demande perçue, qui influence la direction et le rythme du développement technologique. Car Le marché est la source d'idées pour diriger la $R \& D$ et par la suite la $R \& D$ a un rôle réactif (figure ci-desous). Kamien et Schwartz (1975) expliquent que les innovations sont induites par les départements qui traitent directement avec les clients, lesquels signalent les problèmes avec une conception ou suggèrent de nouveaux domaines possibles pour l'investigation. Car les solutions aux problèmes soulevés sont fournies par le personnel de recherche. Dans une certaine mesure, cette approche reflète les pratiques des entreprises qui mettent l'accent sur la planification et la prévision de la création de grands services de planification centralisés, susceptibles de prédire les besoins futurs. 


\section{Processus d'Innovation de deuxième génération (Demande de traction)}

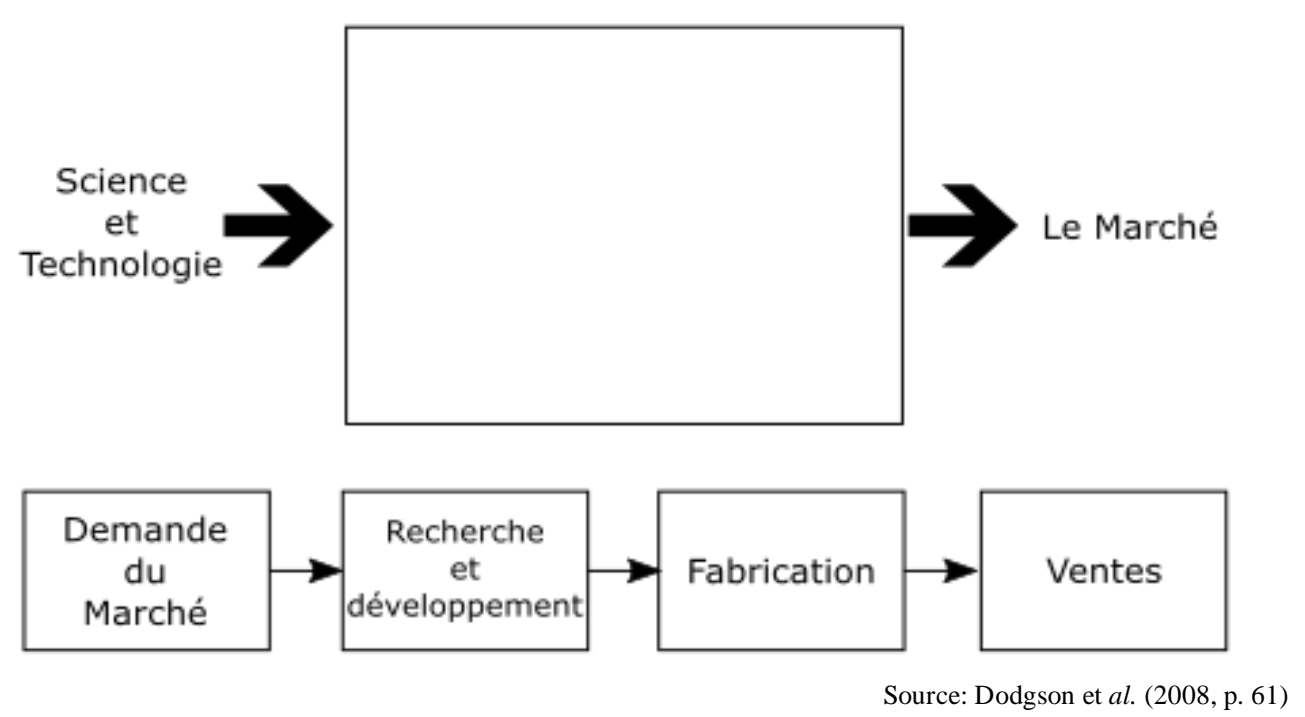

Figure 2.2 La deuxième génération ou”Demand Pull"

\subsubsection{La troisième génération ou du couplage «Coupling»}

Le troisième modèle, le couplage ou le modèle de troisième génération, intégrant à la fois la recherche-poussée et la «Demand pull». Il est axé sur un processus interactif où l'innovation est considérée comme un processus logiquement séquentiel, mais pas nécessairement continu (R Rothwell \& Zegveld, 1985, p. 50). A cet effet, l'accent est mis sur les effets de rétroaction entre les phases aval et amont des modèles linéaires antérieurs. Car les étapes du processus sont perçues comme séparées mais interactives. Le défi de gestion de ce processus implique des investissements importants dans la communication et l'intégration inter-organisationnelle (figure ci-desous). 


\section{Processus d'innovation de troisième génération ...}

\section{(Couplage)}

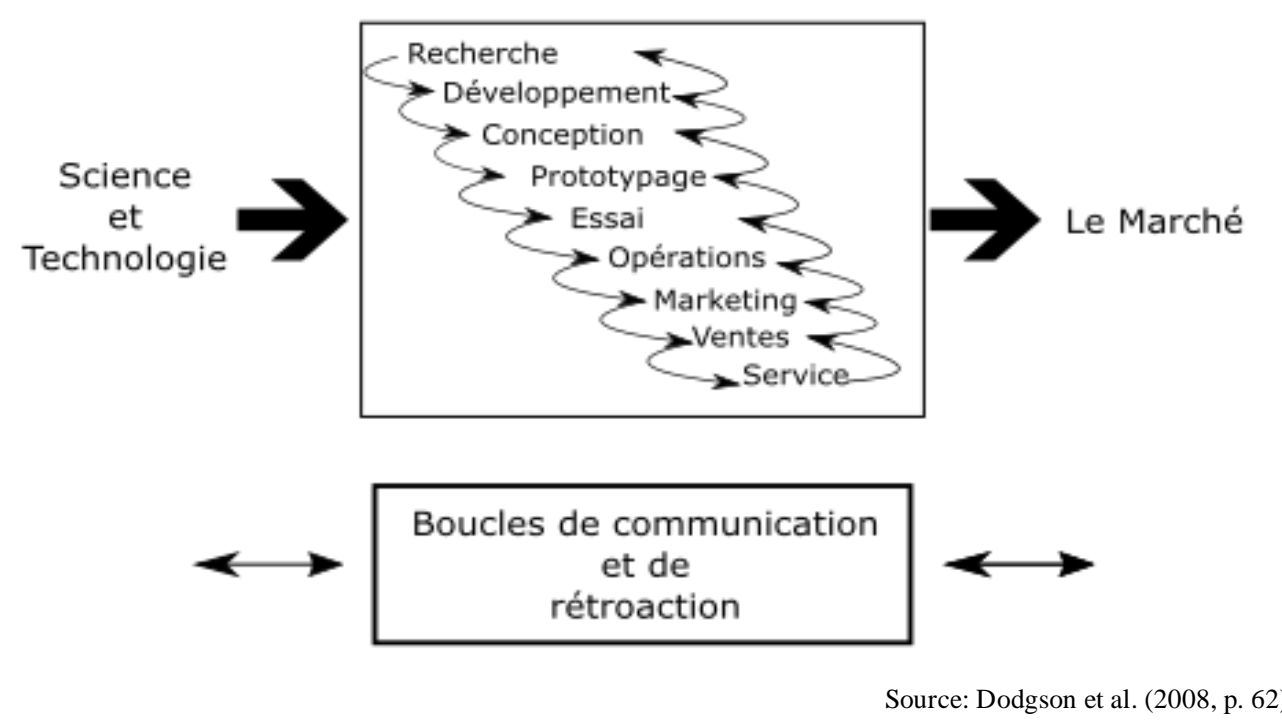

Figure 2.3 La troisièmes génération”Coupling"

\subsubsection{Le modèle de la quatrième génération ou collaboratif «Collaborative»}

Le niveau élevé d'intégration entre les divers éléments de l'entreprise dans l'innovation est repris dans le modèle de quatrième génération, collaboratif ou enchaîné de Kline et Rosenberg (1986, p. 290). Ils montrent les itérations complexes, les boucles de rétroaction et les interrelations entre Marketing, $\mathrm{R} \& \mathrm{D}$, fabrication et distribution dans le processus d'innovation. Ce processus reflète la façon dont l'innovation est impliquée dans la base scientifique et du marché. En revanche elle est accompagnée avec des relations étroites entre les principaux clients et les fournisseurs. En l'occurrence, le modèle est caractérisé par le développement en parallèle avec les équipes de développement et des liens solides avec les fournisseurs (figure ci-desous). 


\section{Processus d'innovation de quatrième génération ... (Collaboratif)}

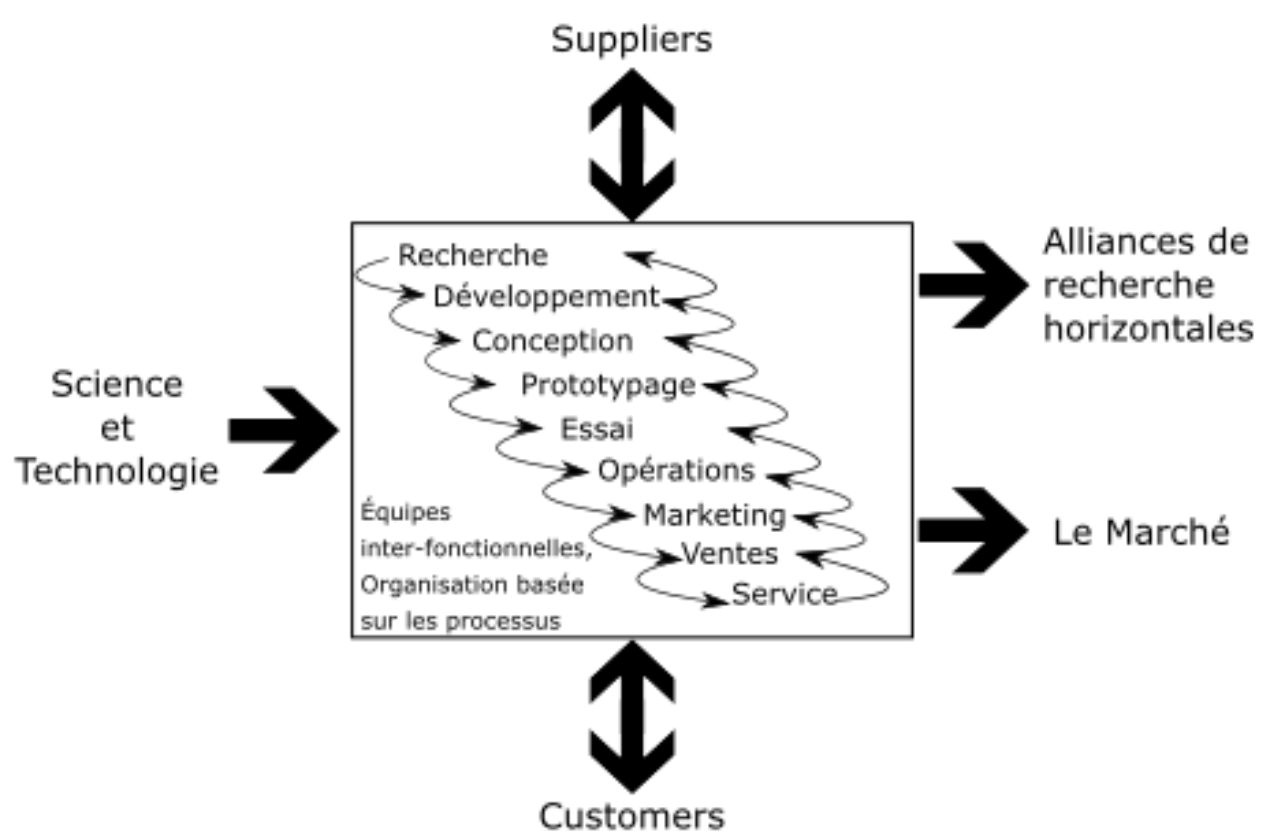

Source: Dodgson et al. (2008, p. 63)

Figure 2.4 La quatrième génération «Collaborative"

2.1.3.5 La cinquième génération ou stratégique et intégrée «Strategic and integrated» Le processus d'innovation de la cinquième génération comprend l'intégration stratégique et technologique croissante entre les différentes organisations à l'intérieur et à l'extérieur de l'entreprise, telles que l'automatisation du processus d'innovation et l'utilisation de nouvelles techniques organisationnelles (Dodgson et al., 2008, p. 63).

A cet effet, le modèle est préoccupé par les formes et les pratiques organisationnelles et les équilibres de compétences qui permettent une flexibilité et une réactivité maximales pour faire face à des marchés imprévisibles et turbulents (figure ci-desous). Rothwell (1992) caractérise cette dernière génération par:

- Intégration des systèmes et mise en réseau du modèle 
- Développement en parallèle entièrement intégré.

- Utilisation de systèmes experts et modélisation de simulation dans R\&D.

- Des liens solides avec les clients de pointe

- Intégration stratégique avec les fournisseurs principaux, y compris le codéveloppement de nouveaux produits

- Liens horizontaux: coentreprises; groupements de recherche collaboratifs; accords de marketing collaboratifs.

- Accent mis sur la souplesse des entreprises et la rapidité du développement (stratégie basée sur le temps).

- Accent accru sur la qualité et d'autres facteurs non tarifaires (Roy Rothwell, 1992).

\section{Processus d'innovation de cinquième génération ...}

(Stratégique et intégré)

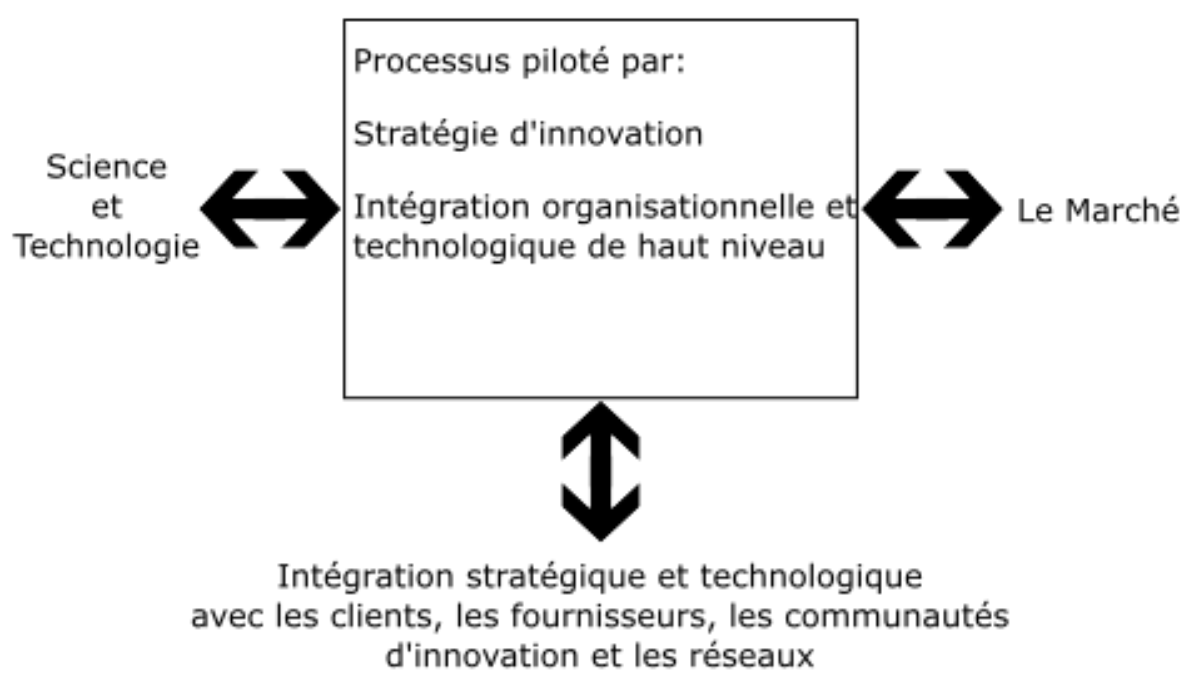

Source: Dodgson et al. (2008, p. 63)

Figure 2.5 La cinquièmes génération"Strategic and Integrated" 
A travers la définition des générations de l'innovation nous pouvons comprendre sur quélle base et conçue l'innovation. Car dans la première génération, l'innovation est conçue du modèle simple basé sur la recheche scientifique sans prendre en compte des exigences et des influences externes ou internes. Dans la deuxiéme génération les décideurs ont introduit l'influence du marché lors de la fabrication. Le troisiémes génération implique tous les départements au sein de l'entreprises afin d'innover. La quatrième génération est une amélioration de la troisiéme car dans celle-ci l'innovation est l'implication de tous les départements au sein de l'entreprises et avec aussi les collaborateurs externes comme les fournisseurs et les clients. Finalement, la cinquiémes génération contient un processus plus performant en matiére de stratégie d'innovation qui implique une haute performance organisationelle afin de faire face á des marchés imprevisibles. A cet effet, notre travail a consisté de comprendre les différents éléments qui participent ou interagisent entre eux afin de déterminer d'abord les caractéristiques du processus d'innovation au sein des entreprises des pays en développment.

A partir de là, la détermination des éléments qui participent à l'innovation nous permetra de nous projeter sur les différentes possibilités de la promotion de l'innovation au sein des ces entreprises. Et ainsi prévoir, les dispositions à prendre afin de booster l'innovation au sein de l'entreprise.

A travers la définition des différentes générations nous considérons que l'innovation se crée non seulement avec les éléments internes de l'entreprise, mais elle nécessite aussi son interconnexion avec le monde extérieur. Dans cette partie nous avons sélectionné quelques modéles d'innovation dans le but de comprendre le processus qui génére l'innovation au sein d'une entreprise.

\subsubsection{Les modèles de gestion d'innovation}

Nous avons séleccioné trois modéles dont l'innovation est générée grâce à l'interaction et la participation des resources et connaissances externes à l'entreprise. Ces modéles sont : premiérement le modèle de gestion totale qui est composé de l'innovation organisationnelle où on exige une coordination totale des composantes internes de l'entreprise avec les changements. Deuxièmement nous proposons le modèle innovatiuon risque qui est la réeéaluation des relations qui existent entre les acteurs externes et internes. Finalement, le modèle innovation ouverte est l'adaptation et l'allocation des sources de connaissance exterieure à l'entreprise et l'interaction avec un plus grand nombre de collaborateurs. L'intérêt de cette sélection est de comprendre l'intérêt et l'influence des collaborateurs externes sur l'innovation.

\subsubsection{Le modèle de gestion totale de l'innovation «totale innovation management» (TIM)}

C'est un modèle qui utilise tous les acteurs qui composent le système innovateur au sein d'une entreprise. Car l'origine de cette théorie est inspirée de la théorie de la synergie de Haken (1984). Elle correspond aux opérations associées de nombreux sous- 
systèmes. Xu et al. (2006) introduit le modèle comme un nouveau paradigme afin d'assurer une totale innovation au sein de l'entreprise en créant de la valeur entre l'innovation technologique et non technologique.

Le modèle de Xu et al. (2006) est composé de l'innovation organisationnelle, innovation institutionnelle et innovation marketing. Chaque composante est reliée avec les autres à travers l'innovation technologique. Ainsi elle est valide à travers le temps et l'espace. l'innovation technologique est considérée comme une source clef pour que les entreprises acquièrent des compétences adaptées aux changements (J. Chen, 2002). Par contre l'innovation marketing signifie l'innovation dans les canaux marketing, les tactiques et les méthodes d'exploitation. En l'occurrence, la structure organisationnelle doit être ajustée en temps utile pour répondre aux besoins d'innovation. Par contre l'innovation institutionnelle est aperçue comme une composante externe parce qu'elle joue un rôle innovateur en matière de règlementation qui affecte la routine organisationnelle, l'évaluation des performances, la rémunération et la sanction du personnel, la formation et la promotion. La figure ci-desous montre cette relation entre les composants du modèle. 


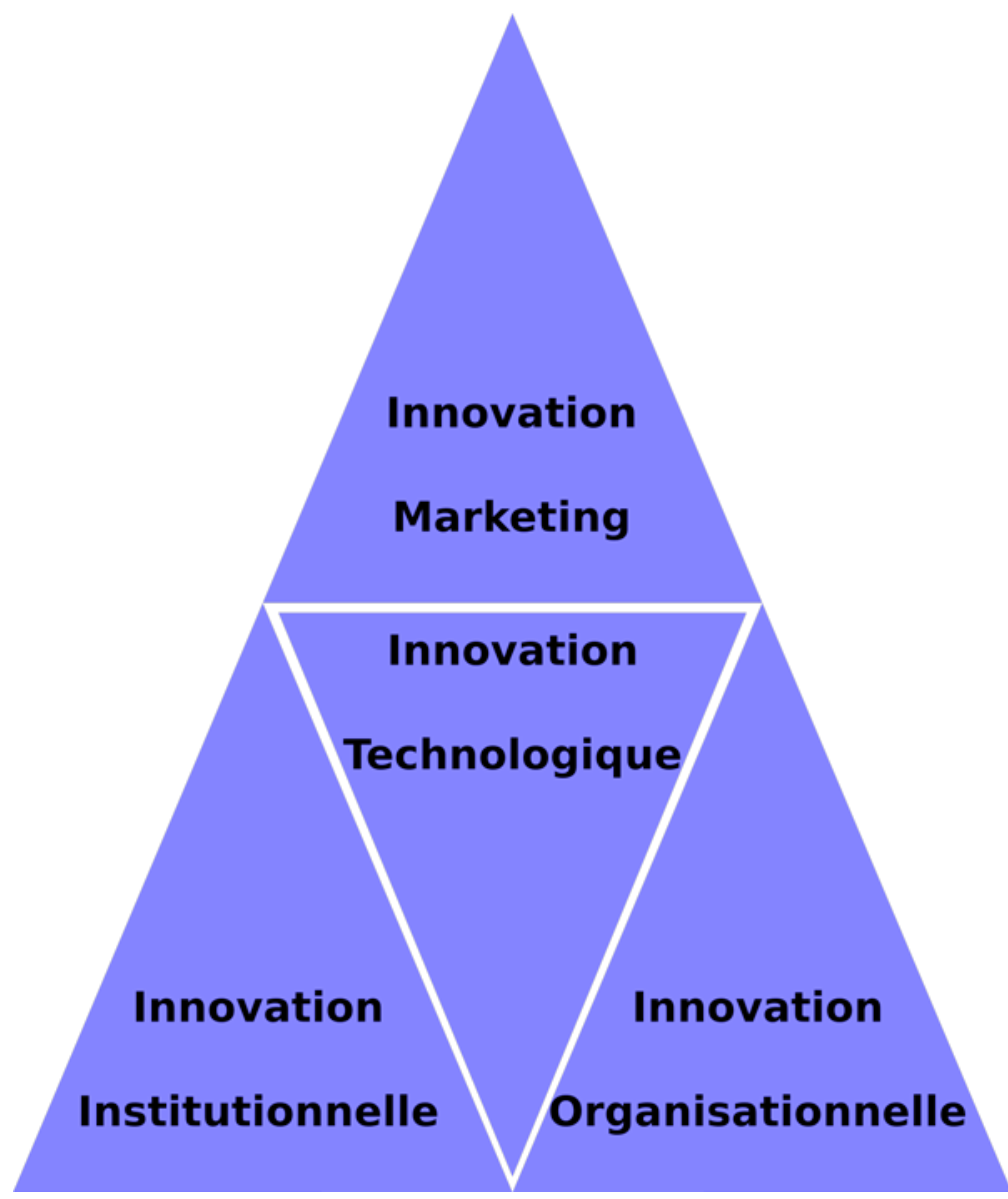

Source: Xu et al. (2006)

Figure 2.6 Les éléments de la gestion totale d'innovation

\subsubsection{Innovation risque}

La théorie de l'innovation risque est inspiré des lacunes de la théorie de l’innovation écosystème.Car l'écosystème est un concept inspiré de l'écosystème biologique auquel on doit penser à la survie des espèces en disparition en conservant un écosystème adéquat (Mirva Peltoniemi, 2006). Cette dernière théorie initie l'innovation de l’écosystème. La problématique de la survie de certaines entreprises ou spécialités au niveau économique demande une réévaluation des relations qui existent entre les acteurs au niveau interne et externe (M Peltoniemi, 2005; Smorodinskaya, Russell, Katukov, \& Still, 2017; Suominen, Seppänen, \& Dedehayir, 2016). En ce qui concerne la théorie innovation risque, Adner (2006) explique cette théorie en considérant que 
l'on ne peut pas innover dans l'écosystème tant qu'on n'a pas une idée sur les risques qui peuvent affecter la performance de l'entreprise et le succès du projet.

A cet effet Adner (2006) propose trois types d'évaluation du risque (figure ci-desous): risques liés à l'interdépendance, risques liés à l'initiative et risques d'intégration. Le risque d'interdépendance est l'évaluation des risques d'interdépendance de la coordination avec les innovateurs complémentaires. En ce qui concerne le risque lié à l'initiative, il consiste à évaluer les risques d'initiatives liés à la gestion du projet central. Finalement, le risque d'intégration évalue les risques d'intégration de la solution adoptée dans toute la chaîne de valeur. Ce processus d'évaluation des risques oblige les gestionnaires à revoir leurs attentes en matière de performance et à repenser par apport à leur plan initial.

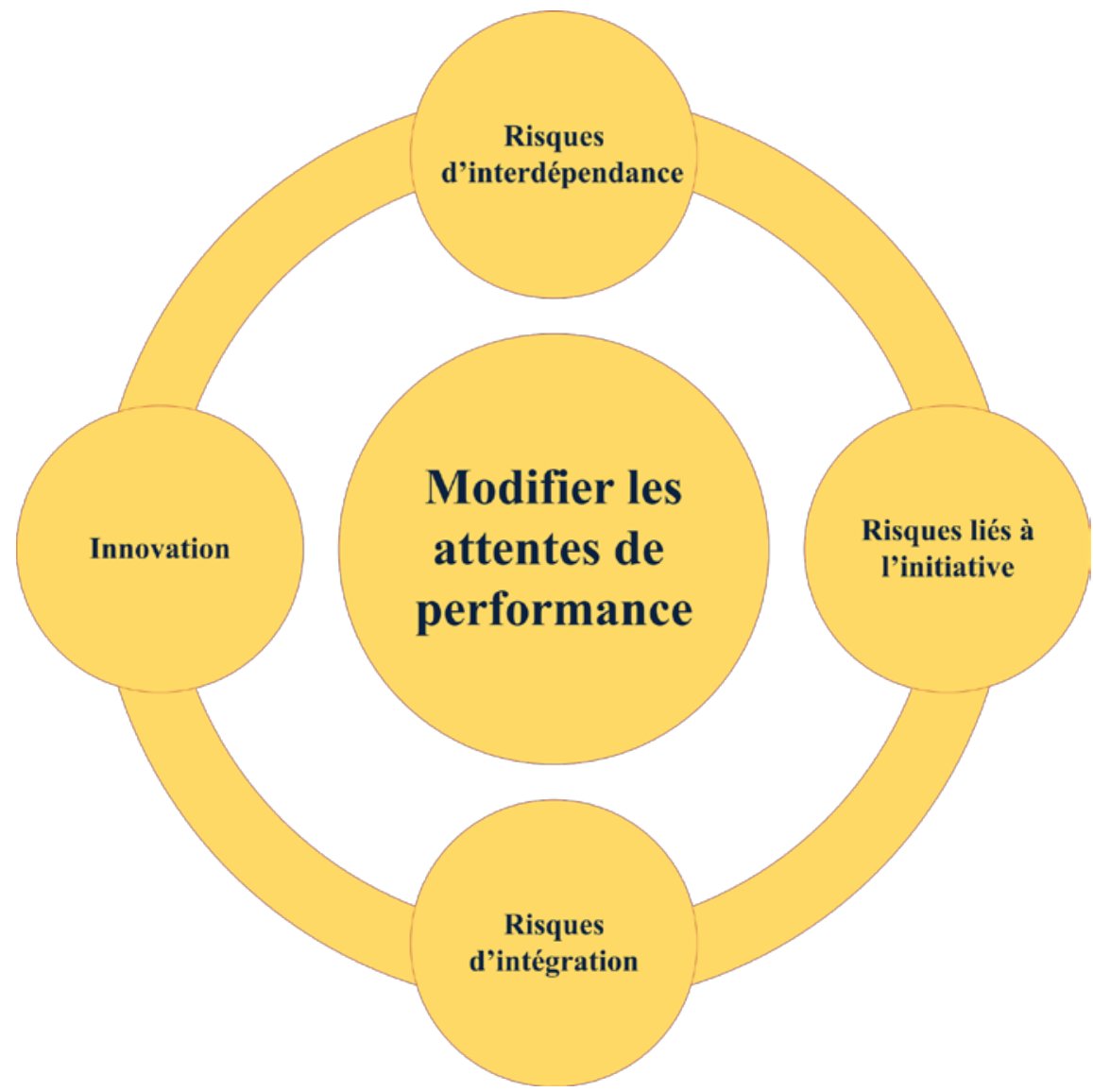

Source: Adner (2006)

Figure 2.7: Le modèle de l'innovation risque 


\subsubsection{Innovation ouverte «open»}

Chesbrough (2003, p. xxv) définit l'innovation ouverte à travers la comparaison entre le système fermé et le système ouvert. Il considère l'innovation fermée comme le passage à l'innovation ouverte (figure 2.8 et 2.9). Car les entreprises ont besoin d'adopter et d'exploiter des sources de connaissances extérieures et de s'engager avec un plus grand nombre et un plus large éventail de collaborateurs. Cependant, l'innovation ouverte se caractérise par des interactions plus fluides entre les activités d'innovation internes et externes, dans lesquelles les idées, les personnes et les ressources circulent dans, autour et hors des organisations.

A cet effet, les frontières entre les activités internes et externes et l'environnement général d'exploitation de l'entreprise sont sensibles; donc il est important d'extraire autant de connaissances de l'environnement extérieur que possible $(\mathrm{H}$. W. Chesbrough \& Crowther, 2006).

Cependant, L'entreprise n'a pas besoin d'exploiter des idées de sa propre recherche pour générer de nouvelles idées, si elle fait les bons raccordements aux réseaux d'innovateurs. Car, construire un meilleur modèle d'affaires pour exploiter de nouvelles idées donnera un meilleur rendement que de se concentrer purement sur l'avantage du premier-moteur. (Cohen \& Levinthal, 1990)

En l'occurrence, Le concept d'innovation ouverte est favorisé dans les entreprises vu le bénéfice qu'il procure à travers des collaborations externes, comme la relation entre l'entreprise et l'université (Bessant \& Venables, 2008).

Chesbrough étale les principes de l'innovation ouverte (2003, p. xxvi) comme suit:

- Les gens intelligents ne travaillent pas pour nous. Nous devons travailler avec des gens intelligents à l'intérieur et à l'extérieur de notre entreprise.

- $\quad$ R\&D externe peut créer une valeur significative, la R\&D interne est nécessaire pour revendiquer une partie de cette valeur.

- On n'a pas besoin d'obtenir l'origine de la recherche pour en tirer profit.

- La construction d'un meilleur modèle d'affaires est meilleure que d'arriver au marché d'abord.

- Si nous faisons le meilleur usage des idées internes et externes, nous allons gagner.

Nous devrions profiter de l'utilisation des autres de notre propriété intellectuelle, et nous devrions acheter d'autres propriétés intellectuelles chaque fois que cela avantage notre propre modèle d'affaires (H. W. Chesbrough, 2003, p. xxvi). 
Chapitre 2 L'état de l'art sur l'innovation, système d'innovation et politique d'innovation

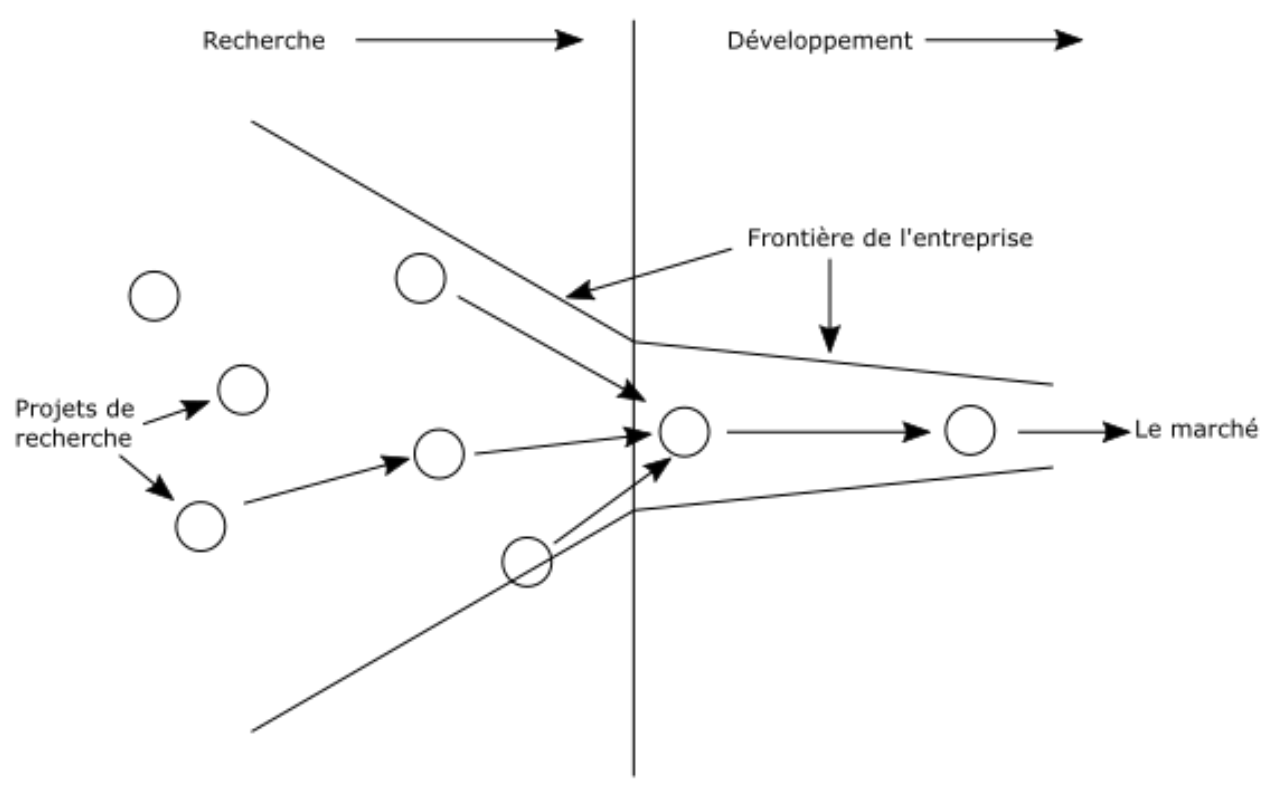

Source: Henry William. Chesbrough (2003, p. xxii)

Figure 2.8 Innovation fermée 


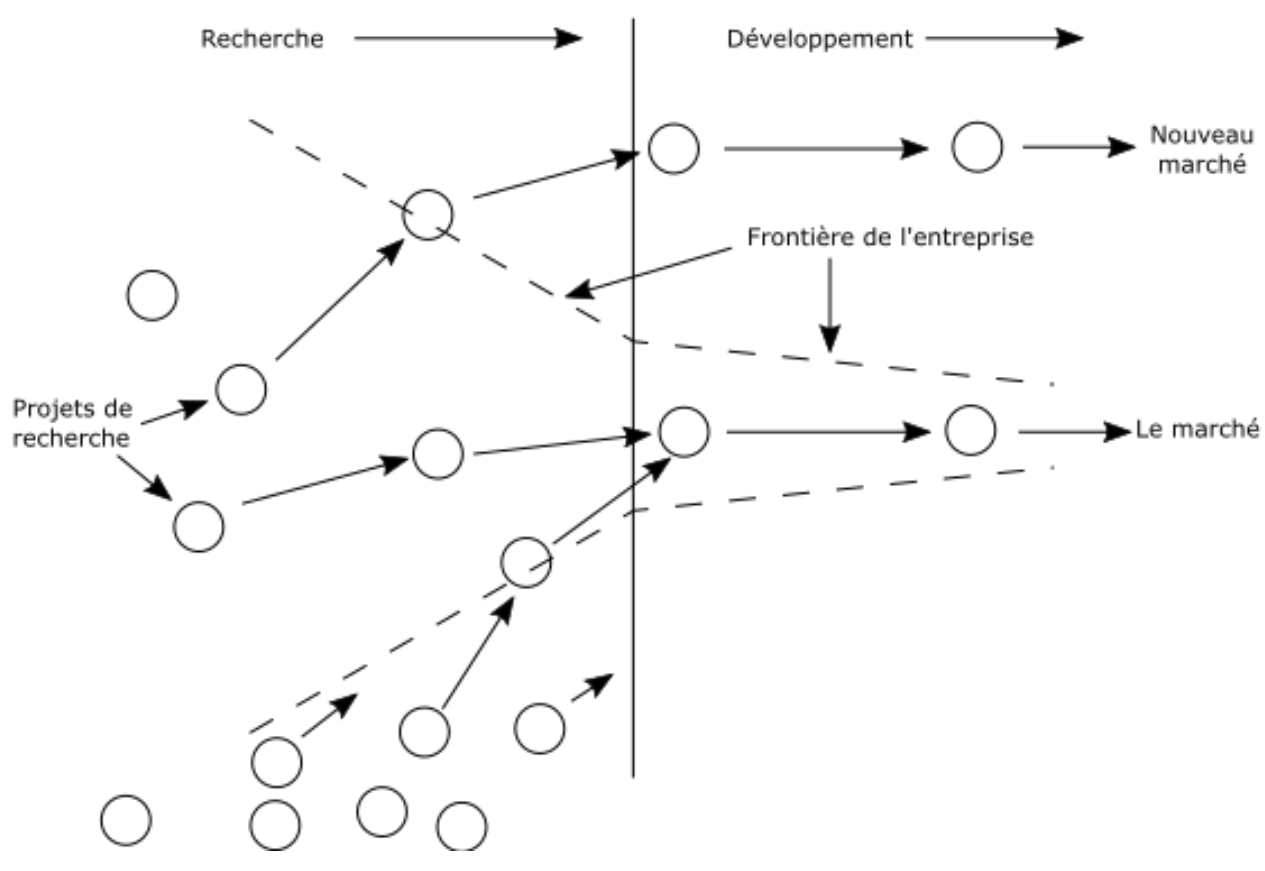

Source: Henry William. Chesbrough (2003, p. xxv)

Figure 2.9 Innovation ouverte

\subsubsection{Conclusion}

La vision de l'innovation au sein de l'entreprise a changé au fil du temps. Avant, le concept d'innovation était fondé sur une théorie basique en relation avec la recherche et développement. En effet le terme d'innovation prend plusieurs postures, de la plus simple à la plus complexe et dans tous les domaines. Dès lors, les résultats innovateurs dépendent des interactions internes et externes de l'entreprise. L'innovation a par ailleurs des caractéristiques atomiques, car les propriétés qui la caractérisent au sein de l'entreprise changent de forme et de couleur ; tout dépend : de l'activité, des interactions au sein de l'entreprise, de l'environnement externe et des caractéristiques géographiques et politiques où elle s'exerce. En conclusion l'innovation ne s'exerce pas toute seule. Elle est connectée et affectée par le système ; ce système est nommé le système d'innovation.

Notre objectif genéral est d'evaluer la politique d'innovation dans les pays en développement. La problématique nous fait penser à l'image macroéconomique de l'état d'innovation au sein d'un pays en développment. Vu la pauvreté des recherches antérieures sur l'état d'innovation au sein des pays en développement, nous pensons que la 
politique d'innovation n'est pas seulement une question d'injecter des instruments ou de promotionner l'innovation mais savoir aussi l'évaluer, comprendre : premiément quelles sont les caractéristiques des résultats d'innovation au sein des entreprises du méme secteur par exemple, deuxèmement, comment on génére l'innovation, savoir si le processus de génération de l'innovation doit être basique ou complexe, finalement savoir, et c'est important, si les résultats d'innovation sont le fruit de collaboration avec des acteurs exterieurs.

En conséquense, l'entreprise générant l'innovation est en collaboration avec les acteurs exterieurs. Cette interaction est chapotée par le système d'innovation.

\section{2. Ètat de l'art sur le Système d'innovation}

Apré avoir défini les types d'innovation et la maniére avec laquelle on peut générer l'innovation au sein de l'entreprise, nous avons conclu que les acteurs externes participent dans la génération de l'innovation au sein de l'entreprise. L'interaction entre les acteurs a un impact positif sur les résultats d'innovation au sein de l'entreprise, car ces acteurs font partie du système d'innovation.

Dans cette partie nous avons essayé de rassembler les définitions et les caractéristiques du système d'innovation. Nous avons consacré une partie sur l'origine et la définition du système d'innovation. Ensuite nous avons introduit le concept 'institutions' afin de mieux comprendre la relation entre le système d'innovation et les différents acteurs et comment ils peuvent influencer le système. Finalement nous avons introduit le concept 'infrastructures économiques' dans le but de mettre la lumière sur les facilités que peuvent offrir les infrastructures au sein du système.

L'objectif est de comprendre dans cette partie la relation des entreprises avec les acteurs qui contribuent à l'innovation et le rôle de chaque composante. Notre travail consiste à prendre en considération tous les éléments qui peuvent afecter les résultats d'innovation au sein de l'entreprise et par la suite, déterminer la position des pouvoirs publics et leur rôle sur la génération des connaissances au sein des entreprises. .

\subsubsection{Le concept du système national d'innovation : origine, dimension et carac- téristiques}

L'objectif de cette partie est premiérement de savoir quels sont les éléments ou les acteurs qui contribuent à l'innovation et cela à travers la définition du système d'innovation. Deuxiémement, nous allons montrer les liens et les impacts institutionnels sur l'entreprise et finalement nous allons déterminer les caractéristiques du système d'innovation. 


\subsubsection{Origine de l'approche système national d'innovation}

Le système national d'innovation est une approche qui a été développée au fil du temps pour mettre en exergue les différences entre les nations. Le concept est apparu d'abord dans la pensée des chercheurs, ensuite il a pris sa place comme une approche que les chercheurs, politiciens et les responsables d'entreprises conscients de la nécessité d'investir pour le développement, ont adoptée. L'approche du système national d'innovation est apparue dans les travaux de Lundvall (1985). Selon Casper et Van (2005) l'approche est apparue dans les années 1980 dans le livre de Lundvall (1985) «Product Innovation and User-Producer Interaction». Lundvall dans son livre explique le système d'innovation à travers la relation entre l'utilisateur et le producteur. L'interaction entre l'utilisateur et le producteur est traduite par la compréhension des connaissances entre l'utilisateur et le producteur. Cette orientation est la manière dont les institutions relient les utilisateurs et les producteurs des connaissances dans le système (B.-Å. Lundvall, 2016, 81).

Ensuite Freeman en 1987 dans son livre «Lesson from Japan» (1987) a comparé l'économie du Japon avec l'économie les autres pays développés comme les États Unis. Freeman a expliqué comment est le système au Japon.

Par conséquent, les travaux sur le système d'innovation ont été entrepris par une collaboration entre Freeman, Nelson et Lundvall (1988; B.-å Lundvall, 1998; 1988) dans le projet de la Fédération internationale des instituts d'études avancées (IFIAS) ${ }^{2}$ sur la technologie et la théorie économique.

Le concept a été introduit par la suite dans le livre de Lundvall (1992) intitulé «National Systems of Innovation: Towards a Theory of Innovation and Interactive Learning». Il a approché le concept apprentissage institutionnel avec le système national d'innovation et la relation entre utilisateur-producteur, dans le but de clarifier la notion du concept système d'innovation, des systèmes nationaux d'innovation et de l'internationalisation. Par ailleurs, le chercheur Nelson introduit le concept dans le livre «National innovation systems: a comparative analysis» (1993). Il analyse le système d'innovation dans les pays à fort, moyen et faible revenu. Finalement le système d'innovation est apparu dans les travaux de Edquist (1997). Son livre «Systems of innovation: technologies, institutions, and organizations» définit le concept et les caractéristiques du système d'innovation.

\footnotetext{
2 L'idée de la Fédération internationale des instituts d'études avancées a été conçue lors d'un symposium Nobel 14 à Stockholm en 1969, intitulé"The Place of Value in a World of Facts". Dans le contexte des travaux sur les systèmes nationaux d'innovation, le programme STI offre l'occasion de collaborer à l'analyse et à la comparaison des structures institutionnelles pour la science et la technologie, ainsi qu'à l'évaluation des interventions politiques appropriées pour accroître le rendement des investissements nationaux. http://ifias.ca/
} 
En l'occurrence, Sharif (2006) d'après son étude qualitative faite à travers les interviews réalisées auprès des fondateurs du concept du système national d'innovation, a démontré que le concept est différent d'un chercheur à un autre. Car, certains d'entre eux cherchent une signification technique, une solution au problème en développant davantage le fondement théorique. Tandis que d'autres ont tendance à redéfinir le concept dont le tracé des frontières reste la préoccupation primordiale

\subsubsection{Définition du système national d'innovation}

Le système national d'innovation est conçu comme une approche. Beaucoup de chercheurs dans le domaine se sont penchés sur la question de la théorisation du système national d'innovation qui reste une problématique, jusqu'à maintenant non résolue. (Casper \& van Waarden, 2005:50).

Le concept introduit initialement par Freeman et Lundvall dans les années 1990 peut être défini comme... «Le réseau d'institutions des secteurs public et privé dont les activités et les interactions initient, importent, modifient et diffusent les nouvelles technologies»..(traduit de l'anglais au français) (Freeman, 1995). Ou bien «Les éléments et les relations qui interagissent dans la production, la diffusion et l'utilisation des connaissances nouvelles, utiles sur le plan économique... et qui sont situés à l'intérieur des frontières d'un état nation».(B.-A. Lundvall, 1992)

Lundvall (1992, p. 2) relie dans sa définition du SNI avec l'approche théorique de l'innovation, dont l'un des points de départ est que l'innovation est un phénomène omniprésent dans l'économie moderne. Il explique que le système national d'innovation comprend toutes les parties et tous les aspects de la structure économique et de l'établissement institutionnel affectant l'apprentissage, ainsi que la recherche et l'exploration y compris le système de production, le système de commercialisation et le système de financement dont il se présentent comme des sous-systèmes dans lesquels l'apprentissage se déroule (B.-Å. Lundvall, 1992, p. 12).

Nelson et Winter (1977, pp. 50-52) suggèrent que les conditions légales, les règles et les normes influeront également de manière significative sur l'inclinaison et la possibilité d'innover d'une organisation. Ils expliquent la complexité des interactions entre les entreprises et les organisations dont le but est de poursuivre l'apprentissage et l'innovation (R. R. Nelson \& Winter, 1977, pp. 50-52)

Edquist (C Edquist, 1997) évoque la question des systèmes d'innovation à travers une combinaison de la théorie de la croissance économique, la théorie du commerce international, la théorie de l'évolution, l'économie de l'organisation, la sociologie organisationnelle, les sciences régionales et les approches institutionnelles émergentes.

En l'occurrence Freeman (1987) définit un système national d'innovation comme «le réseau d'institutions dans les secteurs public et privé dont les activités et les interactions initient, importent, modifient et diffusent les nouvelles technologies» (Freeman, 1987). Car, en décrivant le système national d'innovation japonais, il se concentre sur 
quatre éléments: le rôle du ministère du commerce international et de l'industrie; le rôle de la société $R \& D$; le rôle de l'éducation et de la formation et des innovations sociales connexes et finalement la structure de l'industrie (Freeman, 1987).

McKelvey (1991, p. 136) déduit de son examen des approches NSI qu'il n'y a pas une théorie adéquate pour NSI. Le défi théorique consiste à relier des entités à différents niveaux et à des processus différents. C Freeman \& Soete, précisent (1997) que les interactions systémiques nationales, nécessitent un pont théorique essentiel permettant l'analyse des aspects macro et micro de l'innovation et l'analyse des processus et les changements technologiques.

Le système national d'innovation selon Nelson (1998) est basé sur les réformes institutionnelles et son développement. Il suggère une structure de soutien dans le système afin de comprendre le fonctionnement et le comportement des institutions qui forment le système national d'innovation.

Finalement le système national d'innovation, c'est de comprendre le rôle de la connaissance dans l'économie. Dès lors, le système d'innovation est le fait de comprendre, créer et utiliser la connaissance. A cet effet, la dimension spatiale de l'apprentissage est fondamentale.

Sur le plan pratique le système national d'innovation est l'interaction entre les différentes institutions et organisations. L'interaction inclut le gouvernement, les entreprises et l'université. A cet effet, Le système nous permet de comprendre le comportement innovateur des entreprises..

\subsubsection{Le réseau du système national d'innovation}

Le système national d'innovation est la compréhension des comportements des agents économiques dument les entreprises, les institutions et les organisations (Scott, 1991). Le système national d'innovation nous permet de comprendre les liens et les impacts institutionnels sur le comportement de l'entreprise. Scott (1991) distingue plusieurs types de liens entre les milieux institutionnels et les structures organisationnelles:

Premièrement, L'imposition d'une structure organisationnelle, cela signifie qu'il y a des agents environnementaux suffisamment puissants pour imposer des formes structurelles à des unités organisationnelles subordonnées (Crosby, Bitner, \& Gill, 1990). Deuxièmement, L'autorisation de la structure organisationnelle (Symes, McIntyre, \& Barnett, 2002, p. 105) signifie que l'unité subordonnée n'est pas obligée de se conformer, mais cherche volontairement les attentions et l'approbation de l'agent d'autorisation ou bien peut demander des accréditations par des organismes publics. En particulier dans le domaine de l'essai et l'étalonnage de l'équipement. Troisièmement, l'incitation à la structure organisationnelle. Cela lorsque Les stratégies d'incitation sont appliquées dans les politiques industrielles et technologiques, pour créer des changements structurels dans le comportement innovateur en fournissant des incitations financières aux organisations qui sont prêtes à se conformer aux conditions d'admissibilité 
pour recevoir des fonds sous forme de subventions, de contrats, d'avantages fiscaux (Gompers \& Lerner, 2003). Finalement, l'acquisition de la structure organisationnelle, cela implique le choix délibéré des modèles structurels par les acteurs organisationnels (Weerawardena, O’Cass, \& Julian, 2006).

\subsubsection{Caractéristiques du système national d'innovation}

Le système nationale d'innovation ce caractérise par neuf déterminants qu'on peut les énumérer comme suit (Edquist, 1997: 15-29):

a) Innovation et apprentissage au centre: c'est de transformer des connaissances existantes ou des connaissances nouvelles $(R \& D)$ en produits et processus économiques significatifs. L'innovation est la conséquence de divers types de processus d'apprentissage intégrés dans diverses activités économiques ordinaires (Mothe \& Paquet, 1998, p. 5).

b) Holistique et interdisciplinaire: le système est qualifié de «holistique» car il englobe tous les déterminants de l'innovation dans le système que ce soit dans un contexte national, régional ou sectoriel (Charles Edquist \& Hommen, 1999).

c) Une perspective historique et naturelle: il est expliqué dans la durée qui prend une invention depuis son décalage à son réalisation et puis sa diffusion (Charles Edquist \& Hommen, 1999).

d) Des différences entre les systèmes et la non-optimalité: le SNI est différé par rapport à la structure d'innovation, qui diffère par pays, par région et par secteur. Il est non optimal parce qu'il est en évolution (Charles Edquist \& Hommen, 1999).

e) L'interdépendance et la non-linéarité: Les entreprises interagissent avec d'autres organisations pour innover. Les relations entre les éléments sont extrêmement complexes et souvent caractérisées par la réciprocité, l'interactivité et les mécanismes de rétroaction dans plusieurs boucles, car ils ne sont pas caractérisés par la causalité unilatérale et linéaire (Charles Edquist \& Hommen, 1999).

f) Englobe les innovations de produits et les innovations organisationnelles: Les changements organisationnels sont considérés comme un facteur de développement et de croissance de la productivité dans l'entreprise (Charles Edquist \& Hommen, 1999)

g) Les institutions sont centrales c'est-à-dire les institutions jouent un rôle important dans le système d'innovation à travers les liaisons entre les institutions (Freeman, 1987, p. 1). Lundvall évoque l'importance des institutions dans l'explication de la mise en place institutionnelle (1992, p. 10). Nelson et Rosenberg soulignent les institutions et les mécanismes qui sou- 
tiennent l'innovation technique (1993, p. 1). En ce qui concerne l'institution dans la diffusion de l'innovation, l'infrastructure institutionnelle joue un rôle important. En effet les institutions des structures, favorisent les interactions sociales et l'infrastructure institutionnelle d'un système technologique, soutiennent, stimulent et réglementent le processus d'innovation et de diffusion de la technologie (Carlsson \& Stankiewicz, 1995, p. 49).

h) La diffusion conceptuelle : les systèmes d'innovation sont associés à divers types d'ambiguïtés «ambiguïté conceptuelle», ou dans un langage plus positif «pluralisme conceptuel». L'ambiguïté est positive du moment où le processus de sélection entre les alternatives est naturel pour une nouvelle approche en formation (Charles Edquist \& Hommen, 1999).

i) Un cadre conceptuel plutôt qu'une théorie formelle dans le sens que le system d'innovation est considéré comme approche (Charles Edquist \& Hommen, 1999)

A travers la définition de l'approche du systeme national d'innovation, on comprend que le système national d'innovation n'est pas une théorie et chaque chercheur le définit selon les éléments qui interagissent pour générer l'innovation au sein de l'entreprises. Le systmes d'innovation est considéré comme tout comportement qui affect l'innovation. On peut dire que le systme d'innovation est délimité geografiquement et cela afin de définir le système d'innovation au sein d'une nation. Dont l'approche est spécifique a chaque pays, chaque cas, et situation. En conséquence, l'approche du système national d'innovation est la comprehension du comportement des entreprises, les institutions et les organisations. En déduit à travers les caractéristiques et la liaison entre les composantes que l'instition joue un role dominant dans le système d'innovation. A cet effet, la partie suivantes est consacré à la compréhension du role des institutions dans le system d'innovation.

\subsubsection{Institution et système d'innovation}

Dans cette partie nous allons aborder premiérement le concept 'institution'. Nous évoquerons deuxièmement le rôle des institutions, troisiémement les types d'institutions et finalement la fonction des institutions. A travers ces définitions nous pouvons comprendre les caractéristiques des liens qui existent entre les acteurs et l'impact de chaque composante au moment de la génération de l'innovation au sein de l'entreprise.

\subsubsection{Le concept d'institution}

Dans les définitions du système d'innovation, le concept d'institution joue un rôle dominant dans le système national d'innovation (Freeman, 1987, p. 1). A cet effet, il est défini en termes institutionnels. Il est considéré que les diverses institutions écono- 
miques, sociales et politiques qui influent sur l'innovation sont des éléments centraux du système d'innovation.

L'établissement institutionnel a une incidence sur les processus d'innovation car les institutions affectent les interactions entre les personnes et les habitudes lesquelles constituent l'accumulation des processus d'apprentissage (Charles Edquist, 1997, p. 42).

L'interaction entre les différents individus ou départements se produit au sein des entreprises, entre les entreprises et les consommateurs, entre les différentes entreprises, ou entre les entreprises et d'autres organisations comme les organismes publics (Charles Edquist, 1997, p. 42).

Edquist (1997, p. 42) définit les institutions comme un ensemble d'habitudes, de routines, de pratiques établies, de règles ou de lois qui réglementent les relations et les interactions entre les individus et les groupes. Les interactions entre les individus sont à l'origine du concept d'apprentissage interactif et qui constituent le lien entre les institutions et les innovations.

North (1990) fait la distinction entre les institutions et les organisations. Les deux sont des composantes du cadre structurel qui soutient l'interaction humaine. Les institutions définissent les règles. Les organisations sont des groupes de personnes qui travaillent pour un but ou un objectif commun et ont des intérêts communs. les organisations comprennent de nombreux types d'entités: les organes politiques (partis politiques, conseils municipaux, organismes de réglementation), les organismes économiques (entreprises, syndicats, fermes familiales, coopératives), les organismes sociaux (églises, clubs), et organismes éducatifs (écoles, universités, centres de formation professionnelle) (D. C. North, 1990, p. 5). Les organisations sont influencées et façonnées par les institutions et elles sont intégrées dans un environnement institutionnel ou un ensemble de règles. (Edquist, 1997:59)

Le marché selon North et al. (1990) est une Institution importante du capitalisme moderne car les marchés ne sont pas «purs» mais sont institutionnellement soutenus. Le caractère de ces arrangements institutionnels affecte l'apprentissage interactif et l'innovation. Les institutions peuvent également être empruntées ou importées de l'étranger par les décideurs privés et gouvernementaux. On peut parler «d'imitation institutionnelle» lorsque les institutions sont transférées plus ou moins telles quelles et «l'adaptation institutionnelle» (1990) lorsque des changements sont apportés afin d'améliorer les institutions empruntées ou de les intégrer dans le cadre institutionnel existant.

\subsubsection{Le rôle des institutions}

Chaque chercheur dans son analyse du système national d'innovation a expliqué le rôle des institutions. Casper et van Waarden (2005, pp. 53-54) a résumé la vision des fondateurs de la notion du system national d'innovation à partir des travaux de Mckelvey (1991) et Edquist (C Edquist, 1997). 
Porter (1985) souligne le rôle du gouvernement et les réseaux en comparant entre les entreprises. Il perçoit que la concurrence sur le marché intérieur soutient la structure industrielle.

Freeman (1987, p. 1) se base sur les réseaux d'institutions et compare les nations sur la base de leurs innovations et ajustements dans les institutions sociales. Freeman définit le réseau d'institutions, dans les secteurs public et privé, dont les activités et les interactions initient, modifient et diffusent de nouvelles technologies.

Lundvall (1992) insiste sur l'importance des liens et sur l'interaction au sein des blocs de développement. Les institutions concernées et la structure industrielle forment le système national d'innovation. A partir d'une mise au point empirique il a analysé le développement historique des institutions et des structures de production dans différents pays où il aperçoit que l'établissement institutionnel en tandem avec la structure économique détermine l'apprentissage.

Nelson (1993) suggère que le rôle des institutions est essentiel. Il distingue la différence dans la structure industrielle et la différence dans l'organisation des institutions à travers une étude sur les différences institutionnelles.

Florida (1995) Implique les éléments qui nourrissent l'innovation et leurs relations. Les éléments de base selon Florida (1995) sont: 1) une Infrastructure fabrication, 2) une infrastructure humaine, 3) une infrastructure matérielle et de communication, 4) un système d'allocation de capitaux et un marché financier. Les relations entre les éléments d'un système d'innovation sont les liens qui peuvent être spécifiés en termes de flux de connaissances et d'information, les flux de financement de l'investissement, les flux d'autorité et d'autres arrangements tels que les réseaux, les clubs, et les partenariats. Il conclut d'après une analyse régionale que le rôle de l'institution semble ambigu (Florida, 1995)

Finalement Freeman et Soete (1997) suggèrent que l'institution joue un rôle très important en différences entre les systèmes nationaux d'innovation.

\subsubsection{Type d'institutions}

Stiglitz (2000) distingue institution formelle et institution informelle. Les Institutions formelles sont des institutions caractérisées par la règlementation comme les lois sur la propriété et les brevets, les réglementations gouvernementales de la conduite bancaire, les instructions formelles pour les fonctionnaires du système de service technologique, les règlements et les instructions pour l'installation des équipements électriques par exemple. Par contre l'institution informelle n'est pas visible; elle est constituée par les droits communs, coutumes, traditions, normes de travail, normes de coopération, conventions et pratiques. Les institutions formelles sont visibles et codifiées et les institutions informelles doivent être indirectement observées par le comportement des personnes et des organisations (D. C. North et al., 1991). 
On peut aussi distinguer les institutions de base et les institutions de soutien (Charles Edquist \& Johnson, 1997, p. 56). Les institutions de base sont les règles institutionnelles fondamentales, par exemple, les droits de propriété, les règles de coopération et de résolution des conflits sur le marché du travail et dans les entreprises. Les règles de soutien sont la précision sur certains aspects des règles de base, par exemple les restrictions à l'utilisation de la propriété privée dans des situations spécifiques et les règles régissant le travail en heures supplémentaires dans des industries spécifiques.

Les institutions peuvent être des institutions «soft» et institutions «hard» (D. C. North et al., 1991). Les institutions «dures» sont perçues comme contraignantes et d'une certaine manière policière, et les institutions «douces» sont perçues plus comme des règles de base et des suggestions que comme des commandes qui doivent être obéies. Si une institution est molle ou dure, elle peut dépendre du contexte. Les institutions «Soft» sont plus facilement adaptables et ouvertes aux changements progressifs que les institutions «hard» et, en moyenne, les institutions dures peuvent être plus durables que les institutions douces. L'institution hard est représentée par les institutions de R\&D (Charles Edquist \& Johnson, 1997, p. 56).

Il peut également être fructueux d'identifier les niveaux dans l'établissement institutionnel au sens où il $\mathrm{y}$ a des niveaux dans le système juridique d'un pays, par exemple, les lois constitutionnelles, les statuts et les ordonnances

\subsubsection{La fonction des institutions}

Les fonctions des institutions sont définies selon Edquist et Johnson (1997, pp. 51-55) comme suit:

- Les institutions fournissent des informations et réduisent l'incertitude : l'institution réduit l'incertitude sur le comportement des autres personnes en fournissant des informations ou en réduisant le montant de l’information nécessaire. L'institution agit comme indicateur et régulateur.

- Les institutions gèrent les conflits et la coopération : sans institutions, la régulation des conflits a un impact sur la survie de la société car la coopération serait compliquée et difficile à établir. Le conflit a le potentiel d'être un problème très grave dans le cadre des activités d'innovation.

- Les institutions offrent des incitatifs : il existe des incitations en relation avec l'apprentissage et qui participent aux processus d'innovation. Certains sont de nature pécuniaire comme les régimes salariaux, les impôts sur le revenu, les déductions fiscales et les règles successorales affectent les efforts innovateurs. Aussi Les droits de propriété sur les connaissances et les idées (lois et règles concernant les brevets, les droits d'auteur et les marques) qui affectent la diffusion des connaissances. 
- Les institutions canalisent les ressources vers les activités d'innovation : les institutions influent sur le montant des ressources consacrées à l'innovation. Aussi elles aident à canaliser des ressources vers des domaines spécifiques et à les orienter vers des activités en difficulté

- Les institutions comme obstacle à l'innovation: La capacité de l'économie à générer de la croissance dépend de sa capacité à générer des changements techniques et, en même temps, de sa capacité à adapter et renouveler ses institutions pour soutenir la croissance et l'innovation (Charles Edquist \& Johnson, 1997, pp. 51-55).

Cette partie nous a permis de comprendre: l'importance des institutions dans le système d'innovation, le role qu'elles jouent dans le système d'innovation, les types d'institutions qui peuvent avoir une influence sur l'innovation, et finalement la fonction d'institution.

Nous allons maintenant nous projeter sur la position de ces institutions dans le système d'innovation et cela comme acteur contribuant à l'innovation.

\subsubsection{La fonction et organisation du système national d'innovation}

Dans cette partie nous allons découvrir comment sont organisées les institutions au sein du système. Chaque institution ou organisation a une fonction. Les fonctions difèrent d'une organisation ou institution à une autre. Nous parerons à la fin d les caractéristiques des liens entre les éléments du système.

\subsubsection{Organisations, institution et système national d'innovation}

En liaison avec le changement économique North (1994) détermine, lors de son exposé sur l'évolution économique au fil des temps, les organisations qui peuvent comprendre le système national d'innovation. Il explique la différence entre les composants organisationnels et institutionnels (D. C. North, 1994).

Les organes politiques, tels que les ministères ou les conseils nationaux pour les S\&T; les organes bureaucratiques, par exemple les organismes publics et les bureaux qui mettent en œuvre la politique d'innovation; organismes de réglementation (D. C. North, 1994).

Les normes et la certification qui comprend les organismes sociaux, comme les académies et les associations professionnelles: les organismes éducatifs, comme les universités et les écoles; organismes axés sur le savoir sans but lucratif (D. C. North, 1994).

Les laboratoires gouvernementaux dans le domaine de la défense ou de la santé, ou organismes sans but lucratif ayant des objectifs économiques: entreprises axées sur le profit, y compris les entreprises R\&D, les coentreprises, les consortiums; et des organismes de transition reliant de S\&T aux besoins des entreprises commerciales, telles 
que les centres d'innovation, associés aux chambres de commerce ou aux associations industrielles, ou aux unités de liaison industrielle des universités (D. C. North, 1994).

En l'occurrence, les institutions sont constituées de contraintes formelles, telles que les lois sur les brevets, des critères formels pour l'allocation des ressources à la science, les normes techniques. Ainsi ils sont constitués des contraintes informelles, telles que les normes de comportement, les conventions, les codes de conduite (D. C. North, 1994).

\subsubsection{La fonction du système national d'innovation}

Le système national d'innovation est composé d'un ensemble éléments constitutifs et qui comprennent des groupes d'organisations qui interagissent entre eux. Ces groupes d'organisation partagent certaines caractéristiques (Galli \& Teubal, 1997, p. 346). Après la seconde guerre mondiale, les institutions se sont développées dans une configuration sectorielle ou sous-système relativement bien définie, schématiquement basée sur trois secteurs d'activité R\&D : secteur des entreprises, secteur public et universités, avec des liens relativement faibles entre eux, et un quatrième sous-système d'infrastructure de base comme le bureau de normalisation et Office des brevets.

Chaque organisation dans un bloc de construction exécute un rôle ou une fonction spécifique. Pour les universités, c'est l'enseignement supérieur et la recherche fondamentale; pour les laboratoires gouvernementaux, la recherche axée sur la mission; pour les entreprises, la recherche appliquée et le développement technologique (D. C. North, 1991, p. 346).

On distingue la fonction dure ou hard de la fonction soft (Galli \& Teubal, 1997, p. 346). La fonction dure exigeant des organisations dures qui sont équipées de laboratoires de R\&D. Et les fonctions souples ou soft, qui peuvent être exploitées au sein d'organisations souples qui n’ont pas de laboratoires et ne réalisent donc pas de R\&D. l'implication des fonctions organisationnelles soft est due au rôle crucial que joue ces fonctions (D. C. North, 1991).

- Les fonctions dures et les organisations apparentées comprennent :

o R\&D, impliquant des universités et des organismes publics (gouvernementaux, locaux, mixtes) et sans but lucratif ;

o Des services scientifiques et techniques à des tiers (secteur d'activité et administration publique) par des entreprises industrielles, des centres technologiques, des entreprises de services techniques, des universités, des laboratoires gouvernementaux et des organisations ad hoc (Galli \& Teubal, 1997, p. 347).

- Les fonctions soft et les organisations apparentées sont les suivantes:

o La diffusion de l'information, des connaissances et de la technologie à l'égard des opérateurs économiques et publics agissant à l'interface entre les four- 
nisseurs de connaissances et les utilisateurs ; ces organismes de transition comprennent diverses formes de centres d'innovation et d'unités de liaison dans les universités et les laboratoires publics.

o L'élaboration de politiques par les bureaux du gouvernement, les bureaux d'évaluation de la technologie, les académies, les universités, ad hoc pour a, les comités et conseils nationaux.

o La conception et mise en œuvre d'institutions concernant les brevets, les lois, les normes, les certifications, les réglementations. Ces fonctions sont habituellement exécutées par des organismes publics ou intermédiaires.

o La diffusion/divulgation de la culture scientifique par le biais de musés scientifiques, de centres scientifiques.

o La coordination professionnelle par le biais d'académies, d'associations professionnelles (Galli \& Teubal, 1997, p. 347).

\subsubsection{Caractéristique des liens entre les différents acteurs}

Les organisations sont liées en raison de l'intensité accrue entre les blocs de construction. Trois types de liens sont identifiés (Galli \& Teubal, 1997, pp. 347-348):

- Les opérations de marché, telles que les liaisons en amont et en aval de Hirschmann (1958).

- Les flux unilatéraux de fonds, de compétences et de connaissances (incarnés et désincarnés) au sein d'un institut ainsi qu'à l'extérieur, entre organisations ou entre pays.

- Les interactions, telles que les réseaux utilisateur-fournisseur (Galli \& Teubal, 1997, pp. 347-348).

Les flux et les interactions unilatéraux ont lieu lorsque les marchés n'existent pas ou sont sous-développés ou lorsque le mécanisme du marché n'est pas approprié pour la médiation entre l'offre et la demande, par exemple, les flux de compétences des universités vers le secteur des entreprises pourraient impliquer une externalité. Dans certains cas, les liens institutionnels peuvent évoluer et éventuellement céder la place aux mécanismes du marché ou à une plus grande dose de mécanismes du marché (Galli \& Teubal, 1997, p. 347).

Les liens peuvent être facilités, activés ou incorporés dans une grande variété d'arrangements institutionnels, par exemple, lois, normes et traditions ; règlement d'exécution ; induits par la politique de mesures incitatives et dissuasives ; allocation spécifique et mécanismes de décision au sein des institutions officielles; accords; alliances; accords de coopération et échanges. Interaction entre les opérateurs science et technologie (S\&T) peut avoir lieu dans une variété d'espaces et de dimensions. Leur contexte peut être lié à la géographie, la technologie ou l'industrie. La politique du 
gouvernement est un facteur favorable dans la génération de mécanismes de liaison et d’incitations marché (Galli \& Teubal, 1997).

En résumé, les institutions sont organisées dans le système d'innovation selon la fonction qu'elles occupent et l'effet qu'elles générent sur l'innovation. Car les institutions sont constituées de contraintes formelles, où les organisations sont considérées comme faciliteur ou objecteur à l'activité d'innovation. Ces groupes d'organisation sont classifiés basiquement en entreprises, université et le secteur public. Car chaque composante de ce groupe représente une fonction. Les liens entre ces composantes se caracterisent par des opérations de marché, des fonds, des compétences et des connaissances et les interactions telles que les réseaux utilisateur-fournisseur. A cet effet dans la partie suivavte nous allons entamer en detaille le rôle de chaque composante.

\subsubsection{Les blocs de construction et infrastructure économique du système natio- nal d'innovation}

Le bloc de construction est constitué en fait de trois blocs de base essentiels à la construction du système national d'innovation et un quatrième bloc représentant le nouveau bloc d'infrastructure qui est généralement observé dans les systèmes d'innovation en transition (Galli \& Teubal, 1997).

\subsubsection{Les Universités}

Les universités représentent la composante fondamentale du système d'innovation, avec la responsabilité de fournir l'enseignement supérieur et de réaliser des recherches de base (Meyer-Krahmer, 1997).

Le rôle majeur des universités est de produire:

- La croissance de la recherche multidisciplinaire

- La diffusion de programmes de recherche génériques ou axés sur les missions.

- Une interaction avec le secteur des entreprises comme moyen de surveiller et d'évaluer l'exécution de la recherche fondamentale ainsi que d'orienter le rendement des activités scientifiques.

- La mise en place d'unités d'interface, offrant au secteur des entreprises de nouveaux mécanismes organisationnels pour accéder à la capacité interne, aux compétences et au savoir-faire des laboratoires universitaires, réduisant ainsi les coûts de transaction du secteur des entreprises

- La création d'organismes conjoints de recherche et de développement technologique (Galli \& Teubal, 1997, p. 348). 


\subsubsection{Le secteur public}

Il existe trois principaux types d'organismes publics de R\&D (Galli \& Teubal, 1997, p. 349):

- Les organismes axés sur la mission orientée et les organismes fournissant le soutien scientifique et technique nécessaire aux ministères et autres autorités nationales ou régionales. Par exemples, les instituts nationaux de santé, les agences spatiales, les agences de l'énergie nucléaire, les laboratoires militaires.

- Les organes fondamentaux ou généraux, souvent articulés dans plusieurs centres

- La propriété publique des entreprises (qui fonctionnent généralement dans des secteurs comme les chemins de fer, les télécommunications, le pétrole et gaz, les minéraux) souvent jouent un rôle critique dans les instituts nationaux de statistique, en raison de la part relativement élevée des pays R\&D effectués dans les laboratoires de ces entreprises ainsi que leur rôle dans la définition des normes techniques à un grand nombre d'entreprises (Tassey, 1991).

\subsubsection{Le secteur d'activité}

Le secteur privé ou commercial repose sur les entreprises et leurs laboratoires de recherche et développement, qui jouent un rôle clé dans la recherche et le développement technologique (Galli \& Teubal, 1997, p. 349).

Le processus d'innovation au sein des entreprises subit des changements (Teubal, 1998): le passage d'un processus linéaire basé sur la R \& D à un modèle mettant l'accent sur la pleine intégration des rôles entre les fonctions techniques et le marché et les dispositions externes de l'information et de la technologie (Teubal, 2002).

Ces changements incluent Premièrement, Augmentation de la fréquence et de la variété des liens inter-firme et non médiés par le marché. Deuxièmement, Une intensification des liens entre clients et fournisseurs, généralement aux extrémités de la chaîne de production. Troisièmement, L'extension des entreprises de R \& D, c'est-à-dire des entreprises indépendantes générant des connaissances techniques à des tiers. Finalement, l'extension des sociétés d'ingénierie, de conseil et de services d'information (Galli \& Teubal, 1997, p. 349).

\subsubsection{Les nouveaux composants d'infrastructures}

On peut distinguer deux types d'infrastructure ; une supporte la technologie et l'autre l'innovation:

Les infrastructures de technologie dans les institutions se comportent trois composantes principales : 
- Une infrastructure de base traditionnelle, qui comprend des organisations engagées dans les fonctions souples (tels que les offices de brevets) et les fonctions dures (comme l'infrastructure technologique).

- Une infrastructure technologique (TI), y compris les composants de base et avancés (Justman \& Teubal, 1995), dont les éléments Hard et soft. Les éléments soft comme les unités d'interface et hard pour la réactivité du système en transition.

- Un bloc de développement politique.

En ce qui concerne l'infrastructure d'innovation, elle est caractérisée par la transformation dans la forme dans laquelle les infrastructures hard et soft jouent un rôle de pont entre l'infrastructure de l'innovation catalytique d'interface entre les secteurs NSI et les sous-systèmes.

\subsubsection{Le bloc de développement politique}

La politique joue un rôle critique dans la transition du système national d'innovation en raison des limitations de mécanismes du marché dans l'établissement des priorités et la nécessité d'une coordination planifiée (Radosevic, 1994). En outre, la politique se compose d'un ensemble d'outils déterminés d'une manière exogène associés aux incitations monétaires. Ces outils sont plutôt le résultat d'un processus politique complexe impliquant les priorités pour la conception coordonnée et mise en œuvre des politiques dans les différents domaines et de l'évaluation de la politique (Radosevic, 1999). Les principaux éléments du développement du sous-système de la politique sont (Galli \& Teubal, 1997, p. 352):

- Une vue d'ensemble du système d'innovation, son développement à travers le temps et ses connexions avec la performance économique du pays.

- La génération de la vision à travers des mécanismes et institutions à travers l'étude systématique et l'interaction entre les secteurs, peut conduire à la réalisation des objectifs économiques dans l'ensemble du pays. Cela doit également être basé sur une évaluation systématique des incidences des progrès scientifiques et technologiques.

- Détermination des caractéristiques marquantes des industries en matière de technologie et les politiques scientifiques nécessaires, y compris leurs relations avec les politiques macroéconomiques.

- Une vision coordonnée de l'ensemble de la science, la technologie, la politique industrielle et de leurs interconnexions.

- Une définition des priorités et la reformulation de la politique dans les différentes zones et pour la mise en œuvre des mécanismes de fixation qui sont nécessaires au développement. 
- Le choix de l'approche politique dans chaque domaine.

- La génération explicite des capacités de la politique et des exigences institutionnelles et organisationnelles de ce qui précède.

- Une évaluation systémique des incidences des autres politiques sectorielles par exemple, fiscale, défense, santé et environnement sur les résultats des institutions (Galli \& Teubal, 1997, p. 352)

En résumé, le système d'innovation est composé de trois grandes composantes majeures qui sont : l'université, les entités publiques et le secteur d'activité. Mais il s'est avéré que le système nécessite au fait d'autres entitités qui constituront comme un pont entre les institutions de base.

Le rôle de l'université est omipresent dans le système d'innovation car l'université fornit la technologie, les compétences et évalue le niveau de compétence technologique au niveau des entreprises. Le deuxiément, le secteur public, oriente la recherche et la soutient. Troisémement le secteur d'activité, de méme est une composante qui collabore entre toutes les composantes ainsi qu'entre les entreprises elle-même, et cela dépend de la demande d'innovation dans l'entreprise. L'entreprise générant des connaissances techniques à des tiers. Quatrimement, il existe des infrastructures qui supporte l'innovation et la technologie dont les moyens politiques participent dans l'elaboration et l'orientation de ces infrastructures. Finalement, le secteur politique et l'engin principal du système d'innovation car il a une main sur toutes les composantes du système d'innovation. Ce bloc est consacré principalement à la coordination, la planification, l'evaluation et la mise en œuvre des politiques qui s'adapte aux exigences des composantes.

Nous considérons que chaque composante du système d'innovation est reliée avec les autres, et l'interaction entre elles fait fonctionner la génération car il existe l'infrastructure. Dans la partie suivante nous déterminons le role des infrastructures dans le système d'innovation.

\subsubsection{Infrastructure économique et systéme national d'innovation}

Dans cette partie nous allons définir et caractériser l'infrastructure économique et son impact sur le système d'innovation. De la, le but de cette partie est de mettre en lumiére les différents aspects qui impliquent à la production de l'innovation au sein de l'entreprise scar l'infrastructure économique a des fins communes.

\subsubsection{Le concept infrastructure économique}

L'infrastructure se réfère aux ressources non naturelles et qui sont utilisées collectivement par l'industrie dans la production et la distribution des produits. Cela comprend les systèmes d'approvisionnement en énergie, l'approvisionnement en eau, les systèmes 
de transport (routes, aéroports, ports, etc.) et les systèmes de télécommunications (K. Smith, 1997, p. 90). L'infrastructure peut également impliquer des composantes intangibles sous forme de services du secteur public, telles que les normes techniques, la prestation éducative et les systèmes juridiques (le cadre du droit des contrats). La différence entre ces types d'institutions d'infrastructure réside dans la manière dont elles existent: d'une part, sur la base des décisions d'investissements majeurs, et d'autre par l'évolution des institutions coopératives (K. Smith, 1997, p. 90).

L’infrastructure est considérée comme un ensemble de capacités pertinentes et spécifiques de l'industrie, fournies collectivement, destinées à plusieurs applications dans deux ou plusieurs entreprises ou organisations utilisatrices (Justman \& Teubal, 1995).

\subsubsection{Caractéristiques de l'infrastructure économique}

Il existe des caractéristiques économiques qui distinguent les infrastructures ainsi que les autres catégories de biens d'équipement (K. Smith, 1997):

- Le service d'approvisionnement en électricité doit être construit comme un système complet ou un ensemble de systèmes.

- L'infrastructure est multi-utilisateur dans le sens où il y a beaucoup d'utilisateurs du même système d'approvisionnement, de nombreux utilisateurs de la même route par exemple.

- L'infrastructure est générique et qui constitue une exigence essentielle pour toutes les activités. Elle consiste dans la fourniture de ressources qui est essentielle pour toute activité économique (K. Smith, 1997)

\subsubsection{L'infrastructure de la connaissance}

La production industrielle repose sur les connaissances. La technologie industrielle repose aussi sur des connaissances liées à la transformation des matériels. Ces connaissances peuvent être soit formelles sous forme de connaissances scientifiques ou techniques codifiées, soit tacites incorporées dans le personnel qualifié et les routines techniques. La distinction entre les connaissances formelles et tacites correspond à une distinction entre les connaissances génériques ou accessibles et les connaissances privées (Freidson, 1986, p. 9).

Cette distinction entre la connaissance générique (formelle) et privée (tacite) est essentielle à la théorie de l'innovation, parce qu'elle fait référence aux caractéristiques économiques des connaissances qui sont importantes pour la performance R\&D. Si les connaissances génériques ne sont pas appropriables, les entreprises n'ont aucune incitation à les produire (Tassey, 1991).

La combinaison des connaissances et de la base organisationnelle comme l'infrastructure technologique qui consiste à mettre la science, l'ingénierie et les connaissances technologiques à la disposition de l'industrie privée. Ces connaissances peuvent être 
incorporées dans des formes humaines, institutionnelles ou d'établissement. (Tassey, 1991). Finalement on peut dire que l'infrastructure du savoir public est la combinaison des institutions et le flux des ressources.

\subsubsection{Le rôle de l'infrastructure de la connaissance}

La fonction principale de l'infrastructure économique est la production des connaissances, la production des compétences, les fonctions de régulation et de protection, la création d'entreprises et les fonctions d'accès et de diffusion (Gibbons \& Johnston, 1974). La production et la diffusion des connaissances scientifiques et technologiques: en termes de production des connaissances, le financement de la R\&D public joue un rôle économique essentiel (K. Smith, 1997, p. 95). Les facteurs de base qui affectent les possibilités technologiques comprennent (Gibbons \& Johnston, 1974; RC \& WM, 1987):

- La proximité de la science (avec des questions portant sur la pertinence des sciences fondamentales et appliquées à la technologie de l'industrie).

- Les sources externes de connaissances technologiques que représentent les fournisseurs des équipements de production et les utilisateurs de la production dans les industries, ou les organismes gouvernementaux et les laboratoires.

- La maturité de l'industrie, telle est procurée par la structure d'âge du capitalactions (K. Smith, 1997, p. 98).

Education, formation et compétences : l'éducation est clairement une condition importante pour la production industrielle avancée. Seulement, l'éducation est l'un des principaux éléments des dépenses publiques dans la plupart des économies et cela se traduit par des résultats substantiels, quoique difficiles à mesurer l'impact économique(K. Smith, 1997, p. 99).

Les normes, réglementation et protection des activités techniques: Les activités importantes sont des normes pour les caractéristiques ou la performance des produits dont la protection des connaissances technologique réduit les risques(K. Smith, 1997, pp. 99100).

La création d'entreprises: le rôle économique de l'infrastructure du savoir est l'utilisation des connaissances par les entreprises industrielles dont le rôle est le transfert de technologie entre les infrastructures de base en R\&D et le développement de l'ingénierie dans les entreprises (K. Smith, 1997, p. 101).

Finalement l'accès à la connaissance et la diffusion au point de vue stock des connaissances. De Gibbons et Johnston (1974) discutent sur les mécanismes de l'apprentissage par lesquels la recherche scientifique et l'éducation contribuent à l'innovation industrielle. 
En résumons cette partie, nous apercevons que l'infrastructure économique a des propriétés communes dont est publique . L’infrastructure a deux roles un qui est tangiles. Il correspons au moyen commun qui offre l'etats afin d'offrir des facilités au entreprises de formes générale et sa defaillence peut incuber sur le bon fonctionnement de l'entreprise. Deuxièmement, l'infrastruure au fin intangible qui consiste aus flux des connaissances dont les pouvoir public ont une main deçus en matiére des aquisitions des connaissances et la promotion des compétences. En conséquence, on peut dire que l'innovation existe à plusieurs degrés et niveaux. Cette perception nous permet de délimiter le système d'innovation selon chaque cas et situation.

\subsubsection{Conclusion}

Tout compte fait, le système d'innovation est l'interaction entre les acteurs qui constituent le système d'innovation. Le système national d'innovation est considéré comme la compréhension des comportements des agents d'une part et d'autre part nous permet de comprendre le lien et l'impact institutionnel sur les entreprises. Cepandant les institutions économiques ont une influence sur le système puisque les institutions sont caractérisées par les règlementations et les droits communs. En dernier lieu, l'infrastructure joue un rôle de facilitateur ou de barrières vis-à-vis les entreprises car sont considéré comme des capacités communes.

\subsection{Les types du système d'innovation}

Dans cette section nous allons définir les différents types du système d'innovation qui ont émergé du système national d'innovation. Premièrement, le système sectoriel d'innovation, sa typologie et le régime technologique. Deuxièmement, le système technologique d'innovation, ses éléments et la discontinuité technologique. Troisièmement, le système régional d'innovation, ces formes institutionnelles. Finalement la politique d'innovation.

\subsubsection{Le système sectoriel d'innovation}

Pavitt (1984) a soutenu la répartition sectorielle. Il suggère qu'entre les secteurs il existe des sous ensembles qui participent au développement de l'innovation à travers l'interaction entre elles. Les secteurs sont repartis selon l'activité technologique en trois grandes familles: mécanique, électrique et chimique (Patel \& Pavitt, 1991).

En l'occurrence Guerrieri et Tylecote (1997) ont analysé le niveau de la technologie dans les secteurs en s'appuyant sur la catégorisation de Pavitt (1984). Ils ont distribué les secteurs selon cinq catégories:

- Les sources sectorielles de technologie utilisées dans un secteur concernant les conditions de production dans l'entreprise ou bien celles qui proviennent de l'extérieur comme achat d'équipements et de matériaux. 
- Les sources institutionnelles et la nature de la technologie produite dans un secteur. L'importance des connaissances comme source en intra et extra de l'entreprise ainsi que l'importance des innovations en matière de produits et de procédés.

- Les caractéristiques des entreprises innovantes, en particulier leur taille et leur activité principale.

- Les secteurs de production et d'utilisation de l'innovation selon l'activité principale de l'entreprise. Développement de processus d'innovation dans les textiles par exemple.

- Les secteurs de production de l'innovation, par leur utilisation et par l'activité principale de l'entreprise, sont tous différents. Par exemple, une entreprise principalement en biens d'équipement électroniques développe et produit une innovation en instrumentation pour une utilisation dans la fabrication de véhicules automobiles (Pavitt, 1984).

Mansfield, lui (1988), catégorise l'innovation dans le secteur en matière de cout. Sa catégorie première c'est : la recherche appliquée; préparation des spécifications du produit, prototype, équipements et installations de fabrication et d'outillage, démarrage de la fabrication, Start-up marketing. Cette catégorie est identifiée par le cout de la recherche appliquée et les catégories qui suivent. Elle est assimilée à des placements à faible visibilité.

Dans un sens, on peut caractériser chaque fonction par une variable qui joue un rôle dans l'interactivité du système d'innovation sectoriel (Guerreri \& Tylecote, 1997):

- Interaction fonctionnelle axée sur la production.

- Interaction fonctionnelle axée sur les ventes et le marketing.

- Interaction verticale interclasse, c'est-à-dire la relation entre les directions et le nombre d'employés par entreprise.

- Interaction verticale entre la gestión.

- Interaction externe avec d'autres industries, se caractérise par une concentration sur les biens d'équipement appartenant à des familles électriques, électronique et mécanique.

- Science à la base, se concentre sur le pourcentage de la recherche appliquée.

- La main-d'œuvre techniquement formée, qui constitue le pourcentage de scientifiques et d'ingénieurs dans l'emploi total. Perspicacité spécifique à l'entreprise.

- Le système financier (Guerreri \& Tylecote, 1997). 
On peut dire que le système sectoriel d'innovation est un système d'un groupe d'entreprises activant dans le développement et la fabrication des produits d'un secteur et dans la production et l'utilisation des technologies. Ce système d'entreprises est lié par deux processus: un processus d'interaction et de coopération dans le développement de la technologie des artefacts et un processus de concurrence et de sélection dans des activités innovantes et de marché (Breschi \& Malerba, 1997, p. 131).

Donc les acteurs centraux du système sectoriel d'innovation sont des entreprises privées. La perspective du système sectoriel d'innovation se concentre sur les sources de connaissances et sur le rôle joué par l'espace géographique dans les processus de transmission des connaissances (Breschi \& Malerba, 1997, p. 131). Le système sectoriel n'est pas seulement expliqué par les processus. Car Breschi (1999) évoque la notion du régime technologique pour expliquer le système d'innovation sectoriel.

En conséquence, le système sectoriel d'innovation et le régime technologique Coévoluent entre eux (Breschi \& Malerba, 1997, p. 132). Car, le système d'innovation sectoriel (SIS) est non seulement affecté par des facteurs spécifiques à la technologie, mais aussi par la façon dont les activités innovatrices sont organisées dans un secteur; il est également susceptible de modifier certains paramètres définissant le régime technologique en vigueur. Cependant le cumul des connaissances technologiques est également susceptible d'augmenter avec le temps, car les secteurs deviennent de plus en plus concentrés et les entreprises établies accumulent des compétences et des ressources

\subsubsection{La typologie du système sectoriel d'innovation}

La relation entre le régime technologique et le système sectoriel d'innovation est déterminé selon trois dimensions (Breschi \& Malerba, 1997, pp. 136-141):

- Schumpétérienne dynamique des innovateurs. Cette dimension fait référence aux processus de concurrence et de sélection entre les entreprises. Au niveau empirique, cette dimension se rapporte à diverses mesures de la dynamique industrielle au sein d'un pays, telles que le nombre, la taille et la concentration des innovateurs, leur évolution au fil du temps et le degré de turbulence (natalité et mortalité innovatrices).

- Répartition géographique des innovateurs. Cette dimension fait référence à la localisation géographique des innovateurs et aux activités innovantes au sein d'un pays. Les activités innovatrices peuvent être dispersées au hasard dans un grand nombre de régions ou très concentrées dans quelques régions et régions métropolitaines.

- La connaissance des limites spatiales du processus innovateur des entreprises. Cette dernière dimension fait référence à la localisation géographique des connaissances technologiques et scientifiques ; lesquelles entreprises innovantes sont en mesure de rechercher de nouvelles connaissances. Les connaissances 
pertinentes peuvent résider dans une zone locale ou à l'intérieur des frontières nationales, où elles peuvent circuler à travers les pays ou être disponibles uniquement dans des centres d'excellence spécifiques dispersés dans le monde (Breschi \& Malerba, 1997, pp. 136-141).

\subsubsection{Le concept du régime technologique}

Le concept du régime technologique est expliqué par la force qui participe au façonnement du système sectoriel d'innovation. Le régime est défini par des caractéristiques qui déterminent la distribution géographique des activités innovatrices. Cette notion est développée par Malerba et Orsenigo (1995; 1990). Ils ont élargi le concept du régime technologique par la combinaison de quatre facteurs fondamentaux. Les facteurs du régime technologique (les conditions d'opportunité, les conditions d'appropriation, l'accumulation des connaissances technologiques et la nature de la base des connaissances pertinentes) sont résumés dans la Figure ci desous.

a) Les conditions d'opportunité : ils reflètent la probabilité d'innover pour un montant donné d'argent investi dans la recherche. Plus précisément, quatre dimensions de base de l'opportunité peuvent être identifiées: niveau, omniprésence, sources et variété (Breschi \& Malerba, 1997, p. 134). .

o Le Niveau: Les conditions d'opportunité peuvent être élevées ou basses. Les possibilités élevées offrent de puissants incitatifs pour entreprendre des activités innovantes et dénotent un environnement économique dans lequel les innovateurs potentiels avec une quantité donnée de ressources consacrées à la recherche peuvent trouver des innovations technologiques de manière plus facile.

o La Variété: Un niveau élevé de conditions d'opportunité est associé à une variété potentiellement riche de solutions, d'approches et d'activités technologiques. C'est particulièrement le cas dans les premiers stades d'un cycle de vie de l'industrie.

o Omniprésence: Une nouvelle connaissance peut être appliquées à plusieurs produits et marchés, tandis que dans le cas d'une faible omniprésence des connaissances nouvelles ne s'appliquent qu'à quelques produits et marchés.

o Sources: Les sources d'opportunités technologiques diffèrent. Les conditions d'opportunité sont liées à des percées scientifiques majeures dans les universités ou bien, les possibilités d'innover peuvent souvent provenir de l'avancement des R\&D. Dans d'autres secteurs les sources externes de connaissances en termes de fournisseurs ou d'utilisateurs peuvent jouer un rôle crucial (Breschi \& Malerba, 1997, p. 134).

b) Les conditions appropriation : c'est la possibilité de protéger les innovations de l'imitation et de tirer profit des activités innovantes. Deux dimensions de 
base caractérisent un régime de protection innovante: le niveau et les moyens d'appropriation (Breschi \& Malerba, 1997, p. 134).

o Niveaux d'appropriation : les secteurs industriels peuvent être classés selon des conditions d'appropriation élevées ou basses. Les conditions d'appropriation élevées signifient que les voies existent avec succès pour protéger l'innovation de l'imitation. Et Les conditions d'appropriation faibles dénotent un environnement économique caractérisé par des externalités de connaissances généralisées (retombées)

o Moyens d'appropriation: Les entreprises utilisent divers moyens pour protéger leurs innovations, allant des brevets au secret, aux innovations continues et au contrôle des actifs complémentaires (Breschi \& Malerba, 1997, pp. 134-135).

c) L'accumulation des connaissances technologiques : L'accumulation peut être définie formellement comme le degré de corrélation sérielle entre les innovations et les activités innovatrices. Elle représente la probabilité d'innover au temps $t+$ /conditionnel sur les innovations au temps $t$ ou sur les innovations dans les périodes précédentes. L'accumulation désigne un environnement économique caractérisé par des continuités pertinentes dans les activités innovatrices. À partir de ces définitions, on peut identifier quatre niveaux: cumul au niveau technologique, au niveau de l'entreprise, au niveau sectoriel et finalement au niveau local (Breschi \& Malerba, 1997, p. 135)

o Le niveau technologique: se réfère aux caractéristiques spécifiques des technologies et à la nature cognitive des processus d'apprentissage .

o Le niveau de l'entreprise : L'accumulation au niveau de l'entreprise se produit lorsque la continuité des activités innovantes dépend fortement des compétences de certaines entreprises. Ce type d'accumulation est lié à la dimension organisationnelle ou à la taille de l'entreprise.

o Le niveau sectoriel: L'accumulation au niveau de l'industrie est perçue dans le cas où l'appropriation de l'innovation est faible la diffusion des connaissances à travers les entreprises du secteur est large.

o Niveau local : Ce type d'accumulation peut être lié aux compétences technologiques et aux capacités innovatrices des entreprises situées dans une zone géographique spécifique. Une forte accumulation dans des endroits spécifiques est plus susceptible d'être associée à des conditions d'appropriation faibles et à des débordements de connaissances localisés dans l'espace (Breschi \& Malerba, 1997, p. 135).

d) La base des connaissances pertinentes : les secteurs diffèrent en matière de propriété de la connaissance tout dépend de l'activité de l'entreprise. Deux ca- 
ractéristiques de la base des connaissances: nature de la connaissance et moyens de transmission et de communication des connaissances (Breschi \& Malerba, 1997, p. 136).

o La nature de la connaissance: Les connaissances technologiques impliquent divers degrés de spécificité, de la connaissance tacite, de complexité et d'indépendance comme a souligné Winter (1998) dans ces travaux.

- Générique ou spécifique: dans un secteur, la base des connaissances peut être de nature générique ou spécifique aux domaines d'application bien définis .

- Le degré de tactilité: dans un secteur, la base des connaissances qui soustend les activités innovatrices peut être essentiellement tacite et locale ou codifiée et facilement transférable entre les agents..

- Le degré de complexité: Le degré de complexité est relativement élevé ou faible en ce qui concerne l'intégration de différentes disciplines et technologies scientifiques et techniques nécessaires à des activités novatrices, variété de compétences (R\&D, équipement de fabrication, ingénierie, production et commercialisation)

- Le degré d'indépendance: les connaissances pertinentes aux activités novatrices peuvent être facilement identifiables et isolées ou plutôt faire partie d'un système plus vaste (Breschi \& Malerba, 1997, p. 136).

e) Moyens de transmission des connaissances: caractérisés par les moyens de transmission du savoir en direct ou face à face, l'enseignement et la formation personnels et la mobilité des personnels (Breschi \& Malerba, 1997, p. 137). 
Chapitre 2 L'état de l'art sur l'innovation, système d'innovation et politique d'innovation
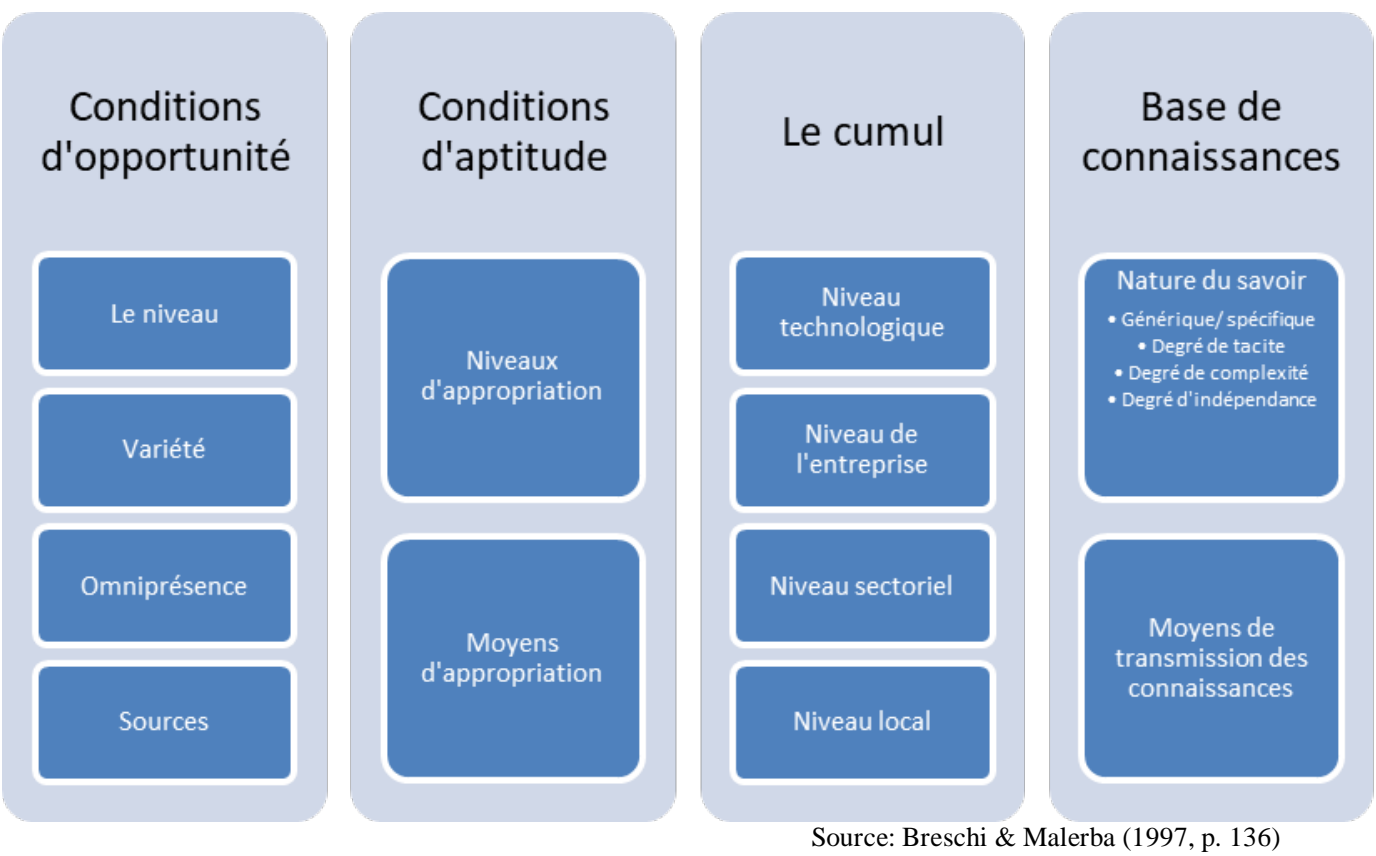

Figure 2.10 Les facteurs du régime sectoriel

f) La capacité d'absorption technologique: Le concept de la capacité d'absorption technologique est défini comme «la capacité d'une entreprise á reconnaitre la valeur de nouvelles informations externes, á les assimiler, et de les appliquer á des fins commerciaux, est essentielle aux capacité d'innovations» (Cohen \& Levinthal, 1990). Il a été suggéré que celà est en grande partie une fonction du niveau des connaissances préalables de l'entreprise.

Zahra et George (2002) suggèrent qu'il est crucial de créer un avantage concurrentiel. Ils concluent que l'importance des capacités technologiques est fondamentales surtout dans le domaine de l'agglomérations industrielles (Hervas-Oliver, Albors-Garrigos, deMiguel, \& Hidalgo, 2012).

\subsubsection{Le système technologique d'innovation}

\subsubsection{La définition du système technologique}

Le concept a été défini initialement par Freeman et Lundval dans les années 1990: il pourrait être défini comme «le réseau d'institutions des secteurs public et privé dont les activités et les interactions initient, importent, modifient et diffusent les nouvelles technologies» (Freeman, 1995) ou bien «Les éléments et les relations qui interagissent dans la production, la diffusion et l'utilisation des connaissances nouvelles, utiles sur le plan 
économique.. et qui sont situés á l'intérieur des frontières d'un état nation» (B.-A. Lundvall, 1992)

En outre, Un système technologique est défini comme un réseau d'agents interagissant dans un domaine technologique spécifique dans le cadre d'une infrastructure institutionnelle particulière et dans le but de générer, diffuser et utiliser la technologie (Garlsson \& Jacobsson, 1991).

Cela implique que les entreprises doivent mettre l'accent sur des stratégies fermes comme être à jour avec les frontières technologiques, intégration au niveau international avec les entreprises à haut niveau technologique, et réorientation vers une production volumineuse

\subsubsection{Les éléments du système technologique}

Le système technologique est constitué de trois composantes qui jouent un rôle dans le processus de spécification de la technologie (Ellinor Ehrnberg \& Edquist, 1998): la compétence économique, les réseaux et les institutions.

Premièrement, La compétence économique est la capacité d'identifier et d'exploiter commercialement les nouvelles technologies. Elle est concernée par la capacité des entreprises à modifier les routines organisationnelles et la base technologique. Cette capacité peut résider dans une ou plusieurs parties du système technologique. Deuxièmement, Le caractère des réseaux auxquels appartient l'entreprise. en ce qui concerne le type et la quantité d'informations et des connaissances auxquelles l'entreprise peut accéder à partir du système. Ce réseau est concrétisé par les relations entre l'acheteur et le vendeur, l'industrie et le milieu universitaire, ainsi divers types d'établissements de transition. Finalement, Les institutions se réfèrent à des processus qui modèlent le comportement humain et peuvent être tangibles, tels que les systèmes éducatifs, les associations industrielles; ainsi que les éléments intangibles comme dans les pratiques de gestion (Ellinor Ehrnberg \& Edquist, 1998)

\subsubsection{La discontinuité technologique et la dynamique industrielle}

Schumpeter (1942) a identifié les changements technologiques discontinus et les innovations connexes comme les sources de la «destruction créatrice» dans les industries. Une discontinuité technologique est une innovation révolutionnaire. Elle est basée sur les nouvelles technologies, dont les limites techniques sont plus grandes que celles de la technologie dominante précédente. Par contre, les méthodes existantes sont modifiées en faveur des nouveaux et des meilleures méthodes selon Schumpeter (1942).

A travers les caractéristiques utilisateurs fournisseur, Ehrnberg et Jacobsson (1997, p. 337) élaborent un schéma (más adelante) afin de démontrer la relation entre la discontinuité technologique et la diffusion de la technologie. À travers le schéma ils expliquent l'importance de l'emplacement de la compétence économique la plus avancée, le réseau de l'entreprise individuelle entre acteurs, qu'il s'agisse d'un client de pointe ou 
d'une institution. Ils suggèrent de comprendre le type et la caractéristique des ressources qu'une entreprise individuelle peut accéder à partir de son réseau. La dimension spatiale est importante au système technologique selon Ehrnberg et Jacobsson (1997, p. 337). Car La connaissance collective de la région peut également avoir une incidence sur la connectivité.

Par la suite, la rapidité de diffusion entre les utilisateurs et la diversité des réponses des concurrents à la nouvelle technologie est très importante. Si la vitesse de diffusion aux utilisateurs est rapide, les premiers déménageurs peuvent gagner des avantages liés au volume, ce qui rend difficile la récupération de retardataires. Cependant, le nouveau produit se diffuse lentement. Par la suite, une plus grande part des entreprises aura le temps de réaliser la nécessité de changer la technologie et de mettre en œuvre les changements (voir figure ci dessous ).

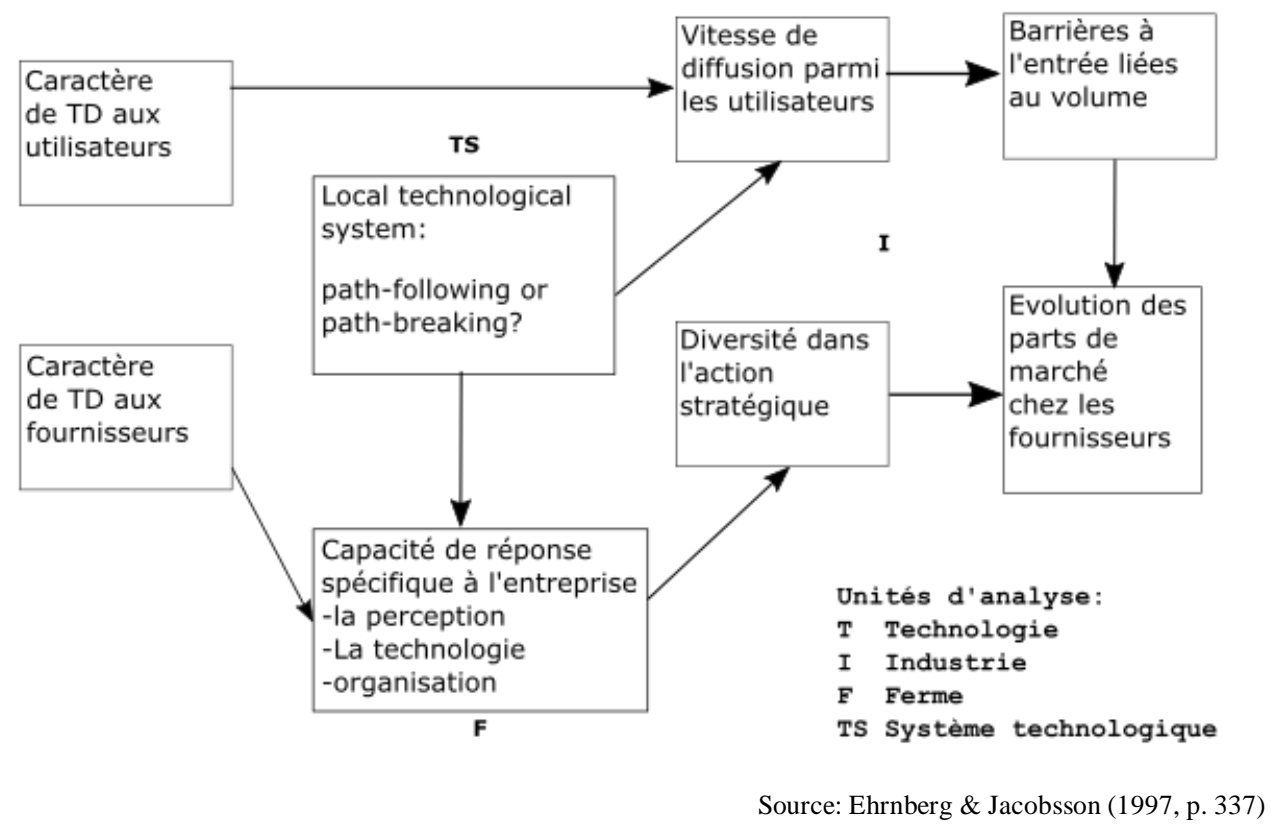

Figure 3.11 La dynamique industrielle et la discontinuité technologique

\subsubsection{Le system régional d'innovation}

\subsubsection{Définition du system régional d'innovation}

Dans le cadre de l'étude des systèmes d'innovation, le système régional d'innovation (RIS) favorise la diffusion rapide des connaissances, des compétences et des meilleures pratiques dans une zone géographique. Le système régional d'innovation est conçu autour des relations économiques, sociales, politiques et institutionnelles qui créent un 
processus d'apprentissage collectif au sein d'un groupe dans une zone précise (Philip Cooke, Gomez Uranga, \& Etxebarria, 1997).

L'origine du concept de système régional d'innovation est dérivée de l'approche du système national d'innovation, Car les chercheurs (Braczyk, Cooke, \& Heidenreich, 1998; P Cooke, 2004; Philip Cooke \& Uranga, 1997; Saxenian, 1994) ont démontré que le système d'innovation au niveau politique et le développement technologique des régions diffèrent. Donc l'importance d'une appropriation d'une vision régionale est primordiale au développement technologique et idem aux décideurs politiques.

Braczyk et al. (Braczyk et al., 1998), dans leur ouvrage «Regional innovation systems: the role of governances in a globalized world», relient en étudiant la stratégie de 14 régions l'approche du système d'innovation régional avec la politique d'innovation de la région. Ils suggèrent que l'innovation dans les régions est vue dans un contexte global et avec une conception diffèrente du développement technologique. Cette controverse doit être ajustée avec les politiques régionales de développement et sociales comme la création d'emploi, les revenus, l'imposition et le bien être social (Braczyk et al., 1998, p. 415).

Le système régional est catégorisé selon Cooke et al. (1997) par des régions caractérisées par un niveau élevé de technologie, par la performance dans les services, la haute performance de la part d'ingénierie, l'ancienneté de l'entreprise avec un potentielle de production et par des régions rurales ou agricoles dans les périphéries. En l'occurrence Tödtling et Trippl (2005a) démontrent que les défaillances régionales en général sont au niveau périphérique dont la connexion est faible entre l'administration métropolitaine et aussi dans les anciennes entreprises.

\subsubsection{Les formes institutionnelles du système régional d'innovation}

Il existe trois formes institutionnelles essentielles au système régional d'innovation afin de faciliter la génération de l'innovation; les cultures financières, éducatives et productives qui peuvent exister pour faciliter l'innovation systémique au niveau régional (Philip Cooke \& Uranga, 1997).

Premièrement, le système financier de chaque région est différent. Car, dans certaines régions, le financement est basé sur des ressources étrangères, dans d'autres sur l'auto financement. Zysman (1983, p. 69) a fait une distinction entre les modes de financement par l'appropriation du concept du système financier: Système orienté vers le marché dont les fonds sont à l'origine du développement du marché du capital, Système à base de crédit dont le gouvernement est régulateur vis à vis des prix. Finalement le modèle des institutions financières qui sont assistées par l'état.

On peut dire que la culture financière est interprétée suivant deux axes selon Cooke et al. (1997). Premièrement, au niveau budgétaire, car la capacité de mobilisation des ressources innovatrice est liée aux disponibilités budgétaires. Deuxièmement, autono- 
mie dans les dépenses dans le cadre de décentralisation politique et finalement l’indépendance fiscale du gouvernement régional.

Et d'autre part du point de vue financement de l'infrastructure dont l'existence de deux types est primordiale au développement de la région (Philip Cooke, Gomez Uranga, et al., 1997). Les deux types d'infrastructure sont: l'infrastructure des télécommunications et les structures qui participent à la création et la diffusion de l'innovation, ainsi que les structures de liaison qui facilitent la communication entre les régions et les pays, comme l'établissement des aéroports.

Deuxièmement, la culture d'apprentissage laquelle est distinguée d'une région à une autre tout dépend de la politique inventive et culturelle d'apprentissage qui règne dans la région. Elle est distinguée par trois formes d'apprentissage: l'apprentissage par l'interaction «the doing by usig innovation (DUI) mode» (B.-A. Lundvall, 2016; B. Å. Lundvall, 2004), l'apprentissage peut être requis à travers le changement institutionnel dans les changements des règles habituelles, et finalement dans les moyens incitatifs à l'apprentissage et la capacité d'absorption.

Lundvall et Johnson (1994) expliquent l'apprentissage à travers du rôle du gouvernement dans l'économie et dans le soutien au processus d'apprentissage. Ils prennent en considération les sujets suivants : les moyens d'apprendre, la motivation à apprendre, la capacité d'apprendre, l'accès à des connaissances pertinentes et finalement apprendre à oublier.

La dernière forme est la relation avec la culture productive. Car une région productive est basée sur les relations dans le système dont la relation entre le system productives des entreprises et le système social au sein de la région (Philip Cooke, Gomez U, et al., 1997). Ce type de relation détermine le degré de développement de chaque région

\subsubsection{Conclusion}

Tout compte fait, le système national d'innovation est á l'origine du concept système d'innovation. Il est délimité par les frontiéres de la nation. Le concept a en réalité évolué à travers le temps, et chaque approche délimite la situation où elle existe. Premiérement, le système d'innovation est sectoriel. Cette approche consiste à étudier les intéractions dans le secteur méme et cela en identifiant les différentes relations qui existent entre les entreprises du méme secteur afin de générer l'innovation. Deuxiémement, le système d'innovation technologique est caractérisé par la diffusion et l'utilisation de la technologie à plusieurs niveaux. Finalement, le système réginal d'innovation est plus proche du concept polititique car la division géographique en régions permet de concentrer les efforts dans une méme région et généralement avec les mémes caractéristiques. En conséquence, chaque type du système a une fin précise et spécifique car les pouvoirs publics et les décideurs politiques ont une main pour facilitier ou compliquer la difusion des connaisssances. Dans le prochain chapitre nous 
expliquons comment les pouvoirs publics peuvent influencer le destin de la génération des connaissances.

\section{4. État de l'art sur la politique d'innovation}

Dans cette partie nous expliquons la polititique d'innovation et les différentes approches ainsi qu'instruments de l'évaluation de la politique d'innovation. Ensuite, nous avons selectionné quelques modèles sur la politique d'innovation et le système d'innovation.

L'objectif dans cette section est de mettre en lumière la relation qui existe entre le système d'innovation au moment de la prise ou de l'élaboration d'une politique. A partir des revues de la literature sur l'innovation et le système d'innovation, nous considérons que pour évaluer la politique il faut comprendre premièrement le processus de l'innovation au sein des entreprises, deuxièmement, déterminer les acteurs et les relations qui existent afin de générer l'innovation, enfin comprendre et détecter le rôle et l'effet de la politique d'innovation. Les recheches antérieures ont été l'objet principal de notre étude.

\subsection{1. État des connaissances sur la politique d'innovation}

\subsubsection{Définition de la politique}

La politique est un concept qui est utilisé depuis longtemps comme un pouvoir imposé seulement par l'état. Car la politique est relative à l'organisation des institutions, à une inclusion d'une opinion, relative à une carrière, l'obtention du pouvoir enfin par rapport aux caractéristiques personnelles comme être habile, judicieux, diplomate et calculateur au profit du bien général (McCool, 1995).

Cependant, la politique joue un rôle important dans la société car elle participe au développement de la nation à partir d'une élaboration et planification d'une politique économique. Par conséquent, l'économie politique est une expression qui inclut la science de la production, de la répartition et de la consommation des richesses (McCool, 1995).

Par ailleurs, l'État Coopère avec les acteurs qui constituent le système à travers une politique publique. Car le rôle des pouvoirs publics est de mettre en place des conditions qui incitent les entreprises à engager les investissements et les efforts d'innovation indispensables à la poursuite du progrès technique (Ocde, 2005, p. 17) afin d'assurer le développement économique et par la suite la création d'emplois.

Dès lors, les pouvoirs publics élaborent une politique à travers un processus qui débute par une planification quelle soit au plan international à travers des coopérations, ou bien à travers des expériences acquises. Ensuite, la conceptualisation de la politique en collaboration avec des politiciens et des experts et consultants, puis la phase de la modélisation de la politique en s'adaptant aux exigences du terrain au plan national et 
finalement sa diffusion au plan régional et territorial ou sectoriel où la politique prend sa forme finale (Peck \& Theodore, 2010).

En effet, la conception finale de la politique est exprimée à travers les instruments politiques qui représentent les facteurs d'appui au développement et les incitations à l'innovation. Ces instruments politiques s'expriment: à travers des lois, des instruments incitatifs au transfert des connaissances et des instruments coopératifs qui sont des instruments soft (M. Bemelmans-Videc, Rist, \& Vedung, 2011; Borrás \& Edquist, 2013; Charles Edquist, 2011).

Finalement, la vision économique politique a émergé à travers les sciences politiques puis par le biais de la politique technologique. Enfin elle s'est globalisée à travers le temps à une vision systémique qui est la politique d'innovation (B.-Å. Lundvall \& Borrás, 2006).

\subsubsection{Politique d'innovation}

L'origine de la politique d'innovation vient de la politique scientifique et technologique. L'intérêt de la politique d'innovation est d'encourager et promovoir l'innovation. Générer de nouvelles connaissances est l'objectif de la politique scientifique ; car les connaissances constituent le moteur de l'innovation et le développement économique (Ocde, OCDE et Eurostat, \& Ocde, 2005). En raison de la complexité et la caractéristique systémique de l'innovation, la politique d'innovation met l'accent sur l'interactivité des acteurs qui représentent le système d'innovation et la diffusion des connaissances au sein du système (Ocde et al., 2005). En l'occurrence, Lundvall et Boras (2006, p. 615) font la distinction entre la science politique, la science technologique et la politique d'innovation en matière d'intérêt et d'occupation.

Brightman (1939) dans son livre met l'accent sur la relation entre la science et l'économie. Il suggère que la recherche scientifique résout des problèmes économiques. En effet, la politique scientifique est associée à la production scientifique dont les instruments (B.-Å. Lundvall \& Borrás, 2006, p. 615) sont:

- Les fonds publics attribués à la recherche et la compétitivité scientifique.

- Instauration des instituts de recherche publics et semi publics lesquels sont les laboratoires, les universités et les centres de recherche.

- Taxe incitative à l'activité de production scientifique au sein des entreprises.

- Promouvoir l'enseignement supérieur.

- La propriété intellectuelle à travers l'élaboration des lois qui incitent à l'innovation ou bien qui protège les connaissances (B.-Å. Lundvall \& Borrás, 2006, p. 615). 
Deuxièmement, la politique technologique est rapportée à la commercialisation de la connaissance spécifique aux secteurs d'activités. Les instruments de la politique (B.-Å. Lundvall \& Borrás, 2006, p. 615) technologique sont:

- Le marché public.

- La contribution des pouvoirs publics à la stratégie du secteur.

- Les instituts : pont qui relie la recherche et le secteur industriel.

- La formation et l'efficacité des compétences.

- La standardisation de la technologie.

- Les prévisions technologiques.

- Benchmarking du secteur technologique politique (B.-Å. Lundvall \& Borrás, 2006, p. 615)

Finalement, la politique d'innovation, son intérêt est plus large. Les instruments politiques (B.-Å. Lundvall \& Borrás, 2006, p. 616) sont:

- Améliorer les compétences individuelles et les capacités d'apprentissage (par le biais du système d'enseignement général et de la formation professionnelle).

- Amélioration de la performance organisationnelle à partir de normalisation et qualité.

- Amélioration de l'accès à l'information.

- Régulation environnementalle et bioéthique.

- Droit des sociétés.

- La régulation de la compétitivité.

- La protection du consommateur.

- Amélioration du capital social dans la région à partir des clusters et districts.

- Intelligence du benchmarking.

- Réflexion intelligente et prévision démocratique (B.-Å. Lundvall \& Borrás, 2006, p. 616).

Selon Edquist (2011), la politique d'innovation rencontre des problèmes quand à la dégradation de la performance du système national d'innovation ou de sa faiblesse. Il attribue le problème politique à:

- L'intensité de l'innovation, c'est à dire comparer les performances dépendant des objectifs structurés. 
Chapitre 2 L'état de l'art sur l'innovation, système d'innovation et politique d'innovation

- La recherche de la cause du problème à travers une analyse détaillée; de nouvelles organisations et institutions pourraient être créées pour développer cette capacité (Charles Edquist, 2011).

\subsubsection{Les différents types de la politique d'innovation}

Edler et Faberge (2017) distinguent trois types de politique d'innovation: premièrement une poltique d'innovation axée sur la mission, laquelle est reliée à l'innovation technologique, deuxièmement une politique orientée vers l'innovation radicale issue de la recherche et developement et troisièmement, une politique axée sur le système d'innovation.

\subsubsection{Les politiques axées sur la mission}

Les politiques axées sur la mission sont définies par Ergas (Ergas, 1986) car le but de cette politique est d'encourager le dévelpppment de la capacité technologique dans le domaine technique, en particulier le domaine de l'armé dans le but de développer certains produits spécifiques. Cette politique prend en considération les differentes étapes du processus d'innovation, de la recherche jusqu'à la commercialisation.

Ergas suggère en comparant les pays de grande puissance technologique que la diffusion de l'innovation joue un role important car la mobilité des employés et des compétences encourage la rapidité de la diffusion de l'innovation. Donc la mission orientée encourage la recheche et développment mais pas la diffusion de la technologie.

En l'occurrence Edquist et Zabala-Iturriagagoitia (2012) définit la mission orientée du point de vue de la politique du marché publique pour l'innovation. Car ils suggérent que la mission orientée est basée sur la satisfaction des besoins humains permettant ainsi la résolution des problèmes de la société. Edler et al. (Edler et al., 2005) classifient le marché public comme : identification des besoins et s'assurer que les utilisateurs sont prêts à y répondre, collecte d'informations sur le marché, spécification de l'appel d'offres, évaluation des offres, attribution des contrats et gestion de l'exécution des contrats.

A l'origine, la mission orientée est militaire ; pour Mowery (1997), c'est de developper les capacités technologiques militaires. Puis, après, la mission orientée a été appliquée dans le domaine de la science d'où l'innovation lineaire. L'innovation lineaire commence par la recherche basic, l'application de la recherche, le developpment et finalement la production et la diffusion, (Godin, 2006, p. 4). A cet effet, Sampat (2012) a appliqué cette vision au domaine de la santé. Le concept de la mission orientée s'est élargi à plusieurs domaines comme la finance et le développment durable (Mazzucato \& Penna, 2015). 


\subsubsection{Les politiques axées sur l'invention}

Selon Edler et Faberge (2017), les politiques axées sur l'invention sont une orientation vers la recherche en focalisant sur la recherche scientifique et en négligeant les résultats. Cette orientation a eu des bénifices par exemple la création des laboratoires de recherche. Cette politique, de Bush d'origine (1945), était devenue populaire après la deuxième guère mondiale. A partir de 1960 on a favorisé la création de nouveaux organismes publics, tels que les conseils de recherche technique; cette démarche a fait la promotion de l'innovation au sein des entreprises (1945)..

\subsubsection{Les politiques axées sur le système}

Les politiques axées sur le système sont orientées vers la compréhension de la relation entre les différents acteurs qui contribuent à l'innovation (B.-Å. Lundvall, 1992). La politique était au départ axée principalement sur la recherche scientifique et puis son concept s'est élargi au niveau des acteurs (B.-Å. Lundvall \& Borrás, 2006). Pour les uns, la politique axée sur le système a une caractéristique d'intéraction (E. S. Andersen \& Lundvall, 1997) et pour d'autres, la génération des connaissances (B.-A. Lundvall, 1992).

Le terme système d'innovation est défini dans un sens global national, sectoriel, régional ou bien tecnologique. Ce type de politiques analyse le système et le degré de participation de chaque acteur afin délaborer les instruments politiques adéquats au développment (Boekholt, 2010).

\subsubsection{Les approches de la politique d'innovation}

Edler et Faberge (2017) distinguent trois approches de la politique d'innovation. Premièrement ils considérent que la politique d'innovation est fondée sur l'échec du marché ; deuxièmement, la politique d'innovation est basée sur le système d'innovation ; troisièmement, la dépendance de l'innovation. Selon Edler et Faberge (2017) ces approches nous permettent de comprendre le comment d'une politique d'innovation.

\subsubsection{L'approche de la politique d'innovation fondée sur l'échec du marché}

L'approche de la politique d'innovation fondée sur l'échec du marché est fondée sur les échecs du marché de 1945. Les décideurs poliriques de cette époque ne considéraient pas les conséquences du non contrôle du marché (Vannevar Bush \& Bush, 1945), car après la deuxième guerre mondiale la majorité des pays puissants ont beaucoup investi sur la guerre et non plus sur la politique. A cet effet, les entrepises se distinguent et se différencient vis-à-vis de la capacité d'innovation et de leur relation avec le marché (Tirole, 2015). En l'occurrence, Jaffe et al. (2005) ont étudié la defaillance du marché à travers les problémes écologiques et proposent une politique orientée vers cette defaillance.ils proposent trois modes de régulation qui sont: les infrastructures, régulateurs sectoriels, ont un impact sur le marché et les concurents; ensuite, les autorités anti- 
confiance qui jouent le role de controleur et enfin, les organisations qui contribuent à l'innovation (Tirole, 2015).

Kleiman et Teles (2006) sugérent trois ètapes pour la défaillance et la non défaillance du marché: la reconnaissance de la portée des écarts par rapport à l'option optimale dans le choix privé, la déférence à l'action volontaire et la reconnaissance de l'omniprésence de l'échec gouvernemental. A cet effet, Edler et Faberge (2017) proposent trois instruments: l'État doit premiérement investir dans la production publique des connaissances, deuxiémement, subventionner la Rcherche et Developpement dans les entreprises privées enfin, établir les régles de la propriété intelectuelle

\subsubsection{L'approche de la politique d'innovation fondée sur le système d'innovation}

L'approche du système d'innovation est apparue dans les années 1980 à travers les recherches de Lundvall (1985) où il explique la relation entre le producteur et l'utilisateur. Cette approche permet aux décideurs de comprendre la caractérisque des relations à travers les connaissances. Puis, de ce concept a émergé le système national d'innovation afin de diférencier entre les pays (E. S. Andersen \& Lundvall, 1997; Freeman, 1995; McKelvey, 1991).

Ensuite, les chercheurs ont approfondi le sujet sur les interactions en détectant que dans le système d'innovation les institutions et les organisations ont un role majeur sur l'innovation (Freeman \& Soete, 1997; McKelvey, 1991; R. Nelson, 1993; Porter, 1985). En outre l'infrastructure a un impact direct sur le système d'innovation (Gibbons \& Johnston, 1974; K. Smith, 1997). En effet, le système national d'innovation a des fonctions qui favorisent la génération des connaissances (Galli \& Teubal, 1997, p. 347).

La politique d'innovation diffère d'une nation à une autre (Charles Edquist, 2006; B.A. Lundvall, 1992), d'un territoire à un autre (PN Cooke, Heidenreich, \& Braczyk, 2004), d'un secteur à un autre (Guerrieri, Innovation, \& 1997, n.d.; Patel \& Pavitt, 1991) et d'un niveau technologique (Garlsson et al., 1991) à un autre, car chaque cas étant spécifique, nécessite à chaque fois une une mise à jours des mèthodes et des outils d'intervention à l'innovation (Kuhlmann, Shapira, \& Smits, 2010).

\subsubsection{Innovation, dépendance à l'égard du cheminement et politiques}

La dépendance de trajectoire est conçue sur la manière du design, la production ou le marketing. Ce concept a été évoqué dans les études sur l'economie évolutioniste. Il existe trois types de domaines d'indépendance de trajectoire : premiérement, la technologie de laguere dure qui comprend la technologie des objets comme les produits et les équipements. Deuxiémement, la base des connaissances, domaine considéré comme l'outil qui relie les individus á la technologie. Finalement, la routine du travail, laquelle est reliée à l'innovation (Coombs \& Hull, 1998). En l'occurrence, Moulaert et al. 
(Moulaert, Martinelli, González, \& Swyngedouw, 2007) expliquent la dépendence de la trajectoire du point de vue de l'innovation sociale.

La dépendance de la trajectoire permet de comprendre les mécanismes des relations au sein des institutions et entre les acteurs (Fuchs \& Shapira, 2005). En outre Strambach la définit comme la relation élastique entre les institutions et les arrangement entre les institutions, car cette flexibilité rend l’innovation dépendante (Strambach, 2008).

\subsubsection{Instrument politique}

Les instruments politiques sont définis comme des techniques de gouvernance qui impliquent les autorités publiques. Ils impliquent, en outre, le comportement des individus dans la société (Howlett, 2005). Les instruments politiques sont considérés comme des moyens de réglementation et de promotion au développement qui permettent au gouvernement de se positionner économiquement (Barber, 1979).

L'implémentation de la politique d'innovation est basée sur les objectifs de la politique. Il doit y avoir une cohérence entre les prises de décision et les buts des politiques. Les étapes du choix des instruments sont basées sur: premièrement, une cohérance entre les objectifs et la cible. Deuxièmement, les outils politiques et les régulations doivent étre consistants et finalemnt doivent être convergents. Donc le choix politique est la décision qu'il faut prendre entre les objectifs à atteindre et les outils appropriés à cet instrument.(Howlett, 2009). Stavins (1996) suggére que les instruments politiques ont un prix; ils sont quantifiables et les deux paramétres doivent être corrélés.

\subsubsection{Evaluation de la politique d'innovation}

La politique d'innovation est définie dans les trois différents axes innovatifs, dans la diffusion et finalement dans l'évaluation (Hildén, Jordan, \& Rayner, 2014). (Magro \& Wilson considèrent que la politique est mixte, car non seulement les décideurs politiques décident sur les instruments de la politique, mais dans le disign de la politique d'innovation, contribuent tous les acteurs (Magro \& Wilson, 2013).

Magro et Wilson (2013) proposent quatre étapes pour l'évaluation de la politique d'innovation. Premièrement, le disign de la politique et l'établisssement des différentes limites des instruments politiques. Deuxièmement, la sélection de la poltique. Troisièmement, l'analyse de l'interaction des acteurs de l'innovation à différentes structures et domaines. Quatrièmement, identification de la politique actuelle et comparaison entre les répercusions politiques et les instruments. Cinquièmement, le design et l'évaluation en intégrant tous les acteurs. Finalement, la considération de l'évaluation à l'échelle holistique où participent tous les acteurs de l'innovation, car la politique d'innovation est efficace lors de l'intégration et la participation des acteurs (Sanderson, 2002). En effet l'évaluation de la politique d'innovation est considérée par rapport à la rationalité, les domaines et les instruments (2013). 
Le système national d'innovation est délimité géographiquement et les types d'innovation sont les dérivés du système national d'innovation. Premièrement, le système sectoriel est l'interaction dans le même secteur. Deuxièmement, le système technologique est l'interaction des agents dans le même domaine technologique. Troisièmement, le système régional est l'interaction entre les membres d'un groupe qui partagent la même culture ou zone.

En consequences, la politique d'innovation est appliquée selon la situation et les defaillences. On peut dire que la politique d'innovation est reajustée et révaluée à chaque fois afin de s'adapter aux exigences de chaque région ou situation. Avant d'entamer le cas de l'évaluation dans les pays en dèveloppment selon la literature nous préférons évoquer diffèrents modèles qui sont reliés au système d'innovation et à la politique d'innovation.

\subsubsection{Les modes de la politique d'innovation et système d'innovation}

Dans cette section nous avons sélectionné les modèles qui expliquent la relation entre les différents acteurs selon plusieurs axes. Premièrement, des modèles qui schématisent le système d'innovation avec les différentes interactions. Deuxièmement, le modèle qui est plus spécifique aux secteurs et qui met en lumière les interactions au sein du secteur. Troisièmement, nous montrons les différentes défaillances du point de vue du système régional. Quatrièmement, le modèle des générations dans le but de comprendre l'évolution de l'innovation à travers les connaissances. Cinquièmement, le modèle nous permettant de comprendre les différents acteurs et l'interaction entre eux à travers l'apprentissage. Sixièmement, nous mettons en exergue la relation entre les instruments politiques et le système d'innovation. Et finalement, nous expliquons l'intervention des décideurs publics dans le système.

\subsubsection{Le modèle du système transitionnel de Galli et Teubal}

Dans leur modèle, Galli et Teubal (1997) proposent une explication de la transition d'une phase simple à une phase complexe du système d'innovation en envisageant les interactions entre les acteurs.

Le système selon Galli et Teubal (1997, p. 353) est construit à partir de «building blocks»; le système est composé des systèmes et de sous-systèmes. En effet, les acteurs sont le secteur commercial, l'université et le gouvernement. La première phase est caractérisée par l'interaction basique entre l'université le gouvernement et le secteur commerciale. La transaction entre le secteur commercial et le marché extérieur est interprété à travers les produits. En ce qui concerne la relation entre les trois acteurs, elle est interprétée à travers des flux unilatéraux, fonds et financements provenants du gouvernement, et des flux de compétences issues de l'université vis-à-vis du secteur commercial. En conséquence le secteur commercial est composé de sous-systèmes qui représentent les grandes et les petites entreprises (Galli \& Teubal, 1997, p. 354) (figure ci-desous) 


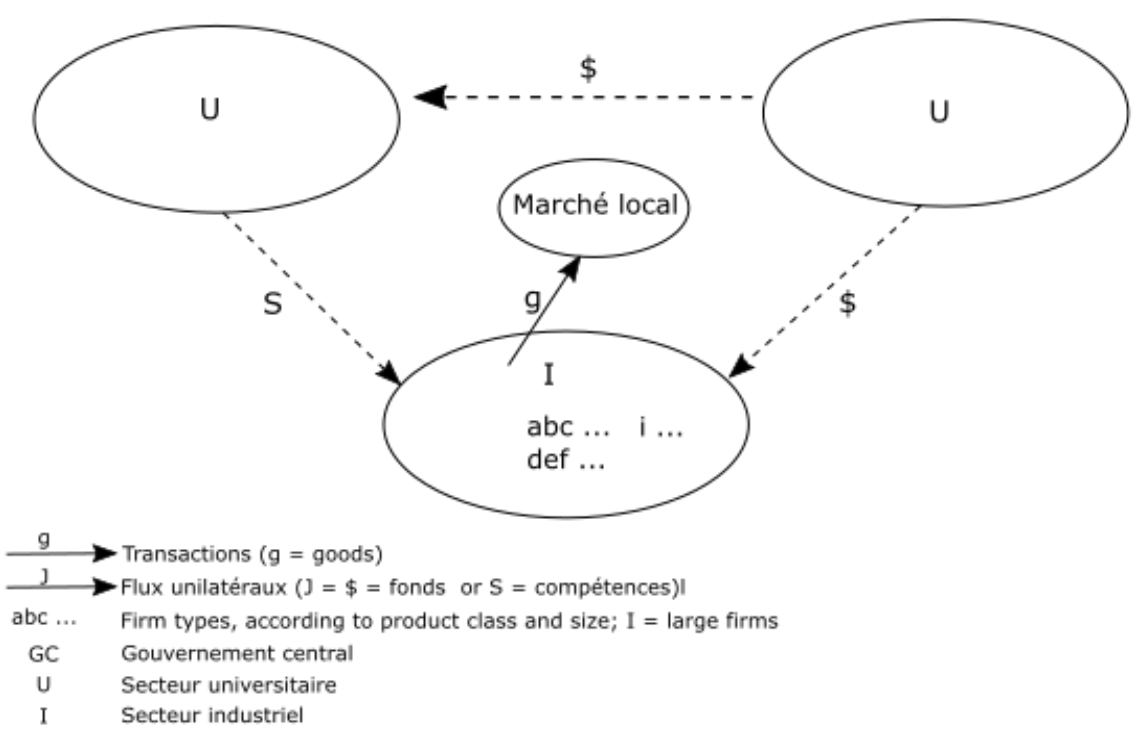

Source: Galli \& Teubal (1997, p. 354)

Figure 2.12 La première phase du système d'innovation

En l'occurrence dans la deuxième phase, le degré d'interaction dans les sous systèmes est beaucoup plus important. En ce qui concerne l'université, une unité d'interface est instaurée afin d'assurer la transaction de la recherche et des flux en matière de technologie et de compétence. Vis-à-vis du secteur commercial une interaction dans les sous systèmes, entre les fournisseurs des produits et les utilisateurs et ainsi qu'une transaction des compétences des grandes firmes vers les petites entreprises. Le système est caractérisé par une intégration des centres technologiques avec les chambres de commerces et les associations des entreprises, car elles sont connectées au secteur commercial, afin d'assurer l'interaction entre les entreprises en matière de compétences et la connectivité entre les entreprises. Les centres technologiques sont aussi subventionnés par l'état (Galli \& Teubal, 1997, p. 354) (figure ci-desous). 


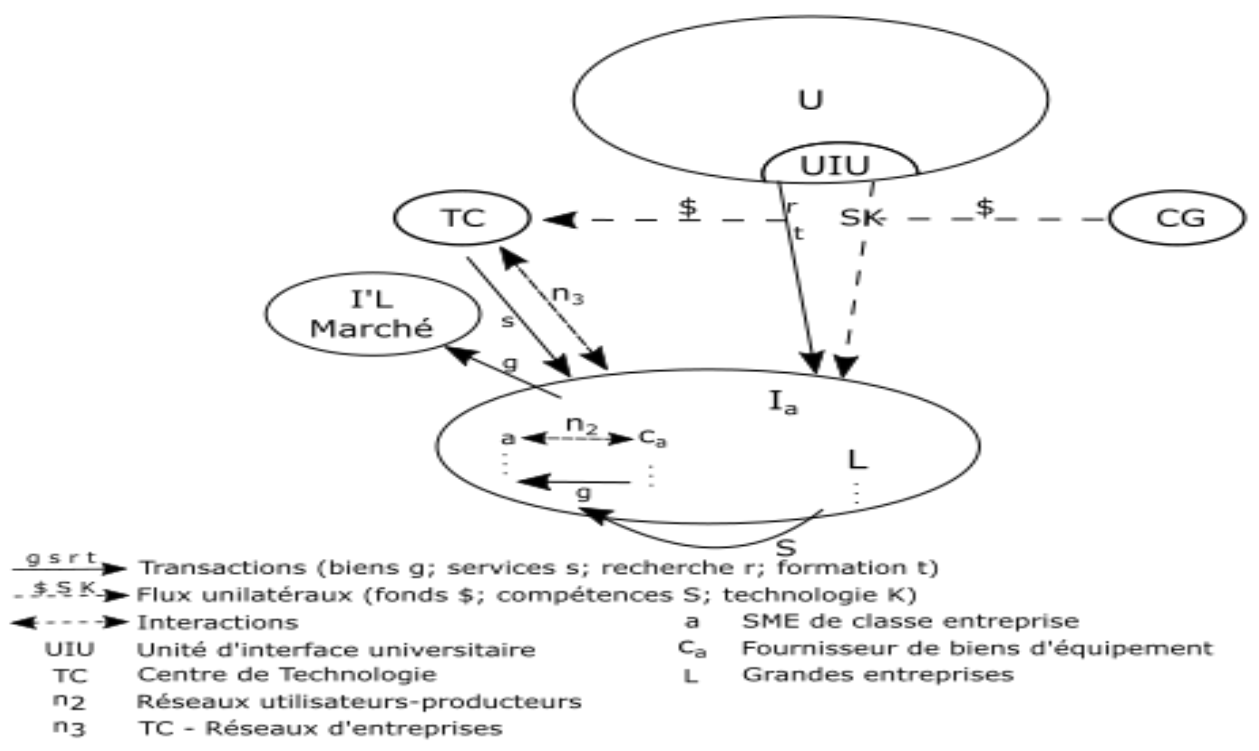

Source: Galli \& Teubal (1997, p. 355)

Figure 4 La deuxième phase du système d'innovation

Finalement la dernière phase et la plus complexe. L'interaction entre l'université et le secteur commercial est directe en ce qui concerne les recherches appliquées en collaboration avec les entreprises et la demande des compétences spécifiques vis-à-vis de l'université. Et d'autre part une demande de prestation vis-à-vis des entreprises au centre technologiques (Galli \& Teubal, 1997, p. 363) (figure ci-desous). 


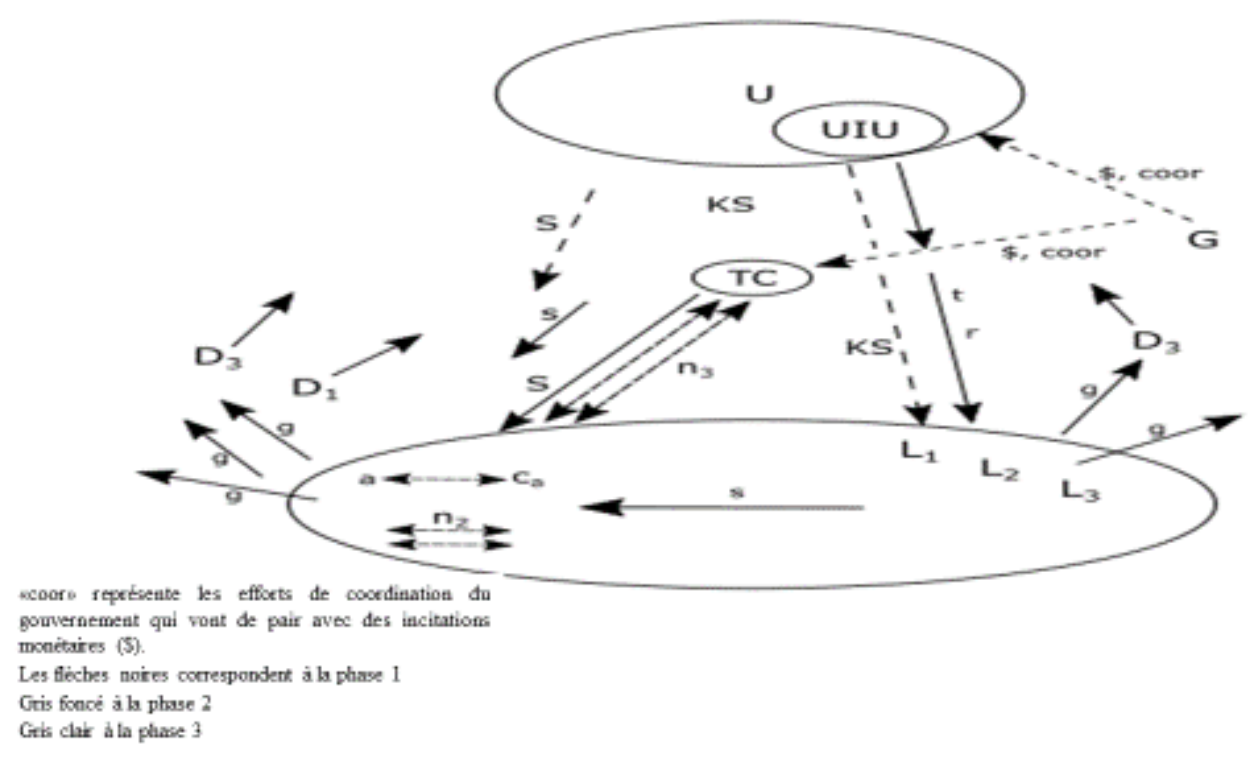

Source: Galli \& Teubal (1997, p. 363)

Figure 54 La troisième phase du système

Finalement, chaque phase en transition est caractérisée par un sous-système. La première phase est l'interaction dans le secteur commercial. La deuxième est la création des centres technologiques et l'interaction entre les entreprises et au même temps une restructuration au sein des secteurs, et finalement la création des interfaces universitaires afin d'assurer l'interaction entre l'université et les entreprises (Galli \& Teubal, 1997, p. 361) (tableau ci-deous). 
Chapitre 2 L'état de l'art sur l'innovation, système d'innovation et politique d'innovation

Tableau 2.1 L'activité et caractéristique des phases de transitions

\begin{tabular}{|c|c|c|}
\hline Les phases & Activité et restructuration du SNI & Sous-système \\
\hline 1 & Spécialisation et la mise en réseaux des entreprises & $A=I^{*}$ \\
\hline 2 & $\begin{array}{c}\text { Création des centres technologiques (TC) et N3 mise } \\
\text { en réseaux (la restructuration du secteur) }\end{array}$ & $\mathrm{B}=1+\mathrm{TC}^{*}$ (+liens) \\
\hline 3 & $\begin{array}{l}\text { Créations des interfaces université intermédiaires } \\
\text { (UIU) et l'émergence des relations (U-I) (La suite des } \\
\text { restructurations du N3 mise en réseaux) }\end{array}$ & $\mathrm{C}=1+\mathrm{TC}+\mathrm{U}^{*}$ (+Lien) \\
\hline
\end{tabular}

\subsubsection{Le modèle vertical d'innovation de Andersen et Lundvall}

Andersen et Lundvall (1997) proposent un modèle vertical où il inclut les relations vendeur et acheteur, les relations entre le marché et les intermédiaires qui soutiennent l'exploitation des innovations et des processus d'innovation. L'intérêt du modèle est de démontrer l'importance des relations novatrices verticales entre les acteurs.

Le modèle est inspiré premièrement de l'approche de la spécialisation du travail de Adam Smith (1976), puis par les travaux de Nelson et Winter (1982) qui expliquent les caractéristiques évolutives de production au sein d'une entreprise à travers un modèle horizontal. En l'occurrence le modèle d'Anderson et Lundvall se focalise sur la dynamique verticale de la division du travail au sein d'un secteur car le processus de production est devisé en nombre de taches spécifiques dans lesquelles les entreprises interagissent.

Le modèle de la division de travail démontre la spécialisation des taches dans chaque étape ainsi que les liens innovateurs qui contribuent à la production et le développement du produit (E. S. Andersen \& Lundvall, 1997) (figure ci-desous) 
Le secteur producteur

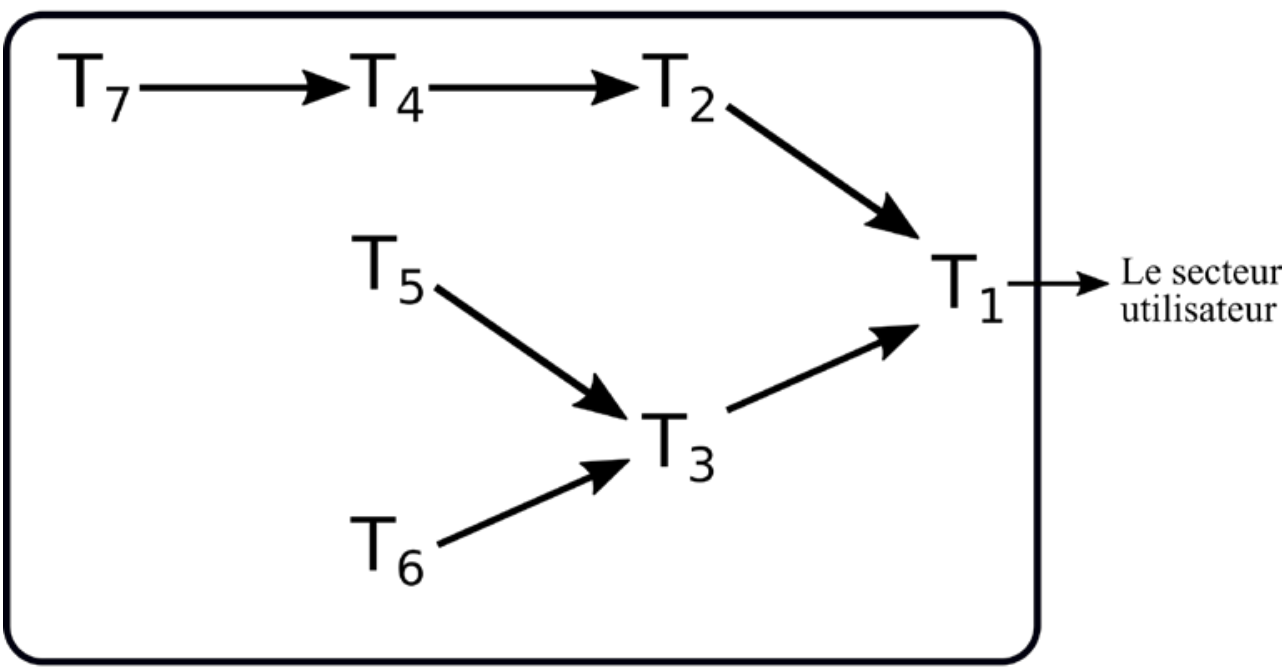

T Les taches

Source: Andersen \& Lundvall (1997, p. 246)

Figure 6 Le system de production simple en relation avec les taches

\subsubsection{Le modèle Franz Tödtling et Michaela Trippl de la politique d'innovation régionale}

Le modèle de Tödtling et Trippl (2005b) est basé sur des études régionales au niveau des industries où ils suggèrent que les niveaux de développement dans les régions diffèrent. En effet, ils expliquent les défaillances du système d'innovation (Kaufmann \& Todtling, 2000). Ils caractérisent les types de contrainte à la génération de l'innovation au sein des entreprises par:

- Un faible niveau de regroupement et une faible dotation en institutions pertinentes" Organizational thinness".

- Un manque d'interaction et un faible réseau d'interaction entre les secteurs"fragmentation".

- Les situations de verrouillage”lock in" (Tödtling \& Trippl, 2005b).

En conséquence ils délimitent chaque contrainte à une région spécifique. Ils relient les contraintes rencontrées au faible regroupement institutionnel aux régions en périphérie, ainsi que le problème de verrouillage de la part des anciennes entreprises par rapport à des entreprises naissantes et finalement le problème d'interaction entre les acteurs est rencontré beaucoup plus dans les régions métropolitaines (Tödtling \& Trippl, 2005b) (figure ci-desous). 
Finalement, à travers le modèle, Tödtling et Trippl (2005b) attribuent la cause de la défaillance du système régional d'innovation à deux phénomènes: le sousdéveloppement organisationnel et institutionnel et aussi l'appropriation relationnelle et le manque d'interaction entre les acteurs.

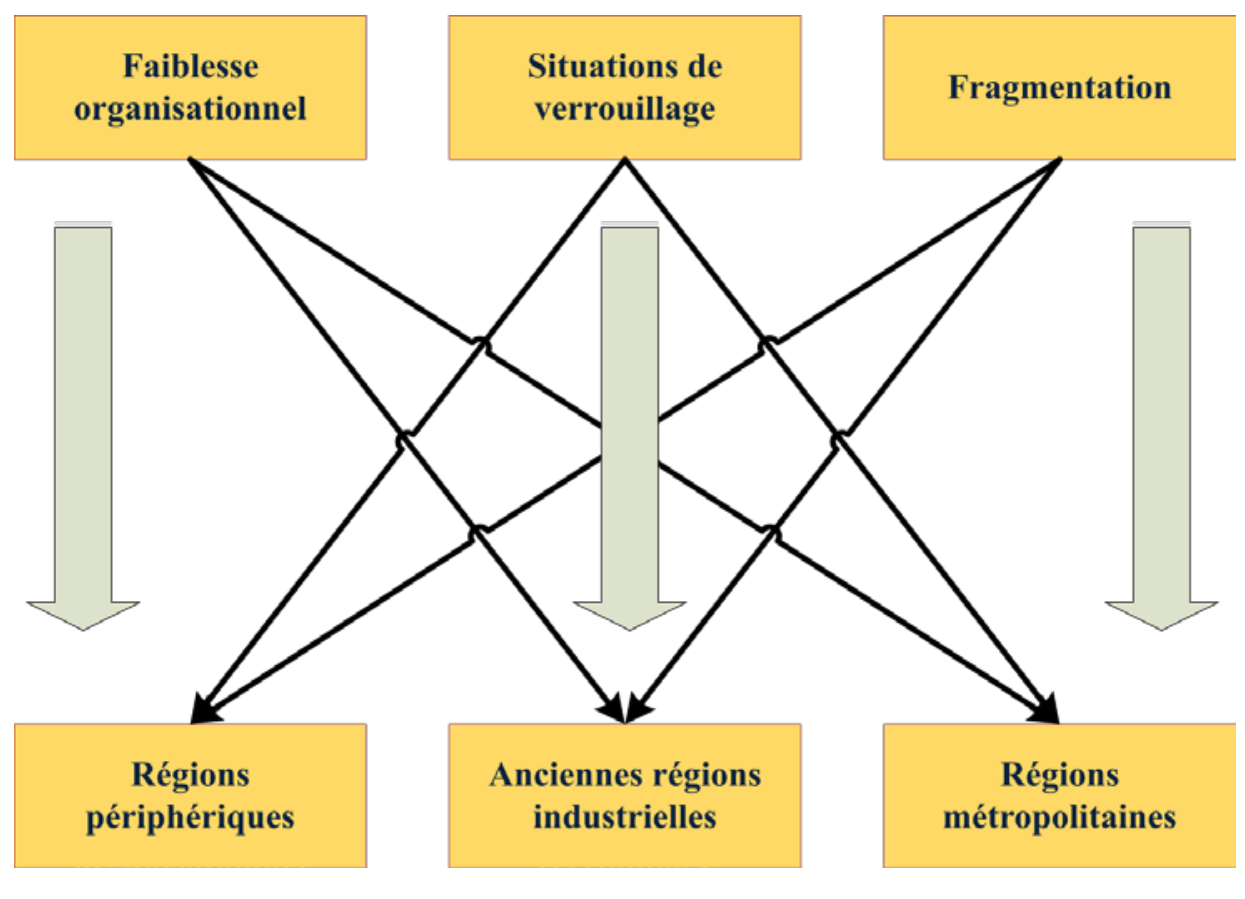

Source: Tödtling \& Trippl (2005b)

Figure 2.16 Les contraintes à l'innovation par région

2.4.6.4 Le modèle de De Lucio, Mas-Verdu et Tortosa des générations de l'innovation La politique du système d'innovation ce base sur trois générations que De Lucio et al. (2010) expliquent à travers une analyse des projets régionaux en Espagne (figure cidesous):

La première génération est basée sur la création des connaissances nouvelles à partir d'un processus d'innovation linéaire de la $R \& D$ à l'expérimentation où se concentre l'offre; à ce niveau la politique de la promotion de la recherche scientifique et du R\&D prime (de Lucio et al., 2010).

La deuxième génération met l'accent sur les instruments de régulation organisationnelle et institutionnelle comme la fiscalité, la propriété intellectuelle (de Lucio et al., 2010). 
Finalement, la troisième génération se focalise sur un système plus complexe où la création des connaissances et la promotion de la génération des connaissances est importante à cette étape (de Lucio et al., 2010).

En effet ils proposent trois politiques à la génération des connaissances. Premièrement identifier les acteurs clés et les impliquer dans le processus d'innovation. Puis développer des interfaces et des organisations pour faciliter la diffusion des connaissances et l'accumulation de l'apprentissage par l'expérience et finalement renforcer les actions de coordination.

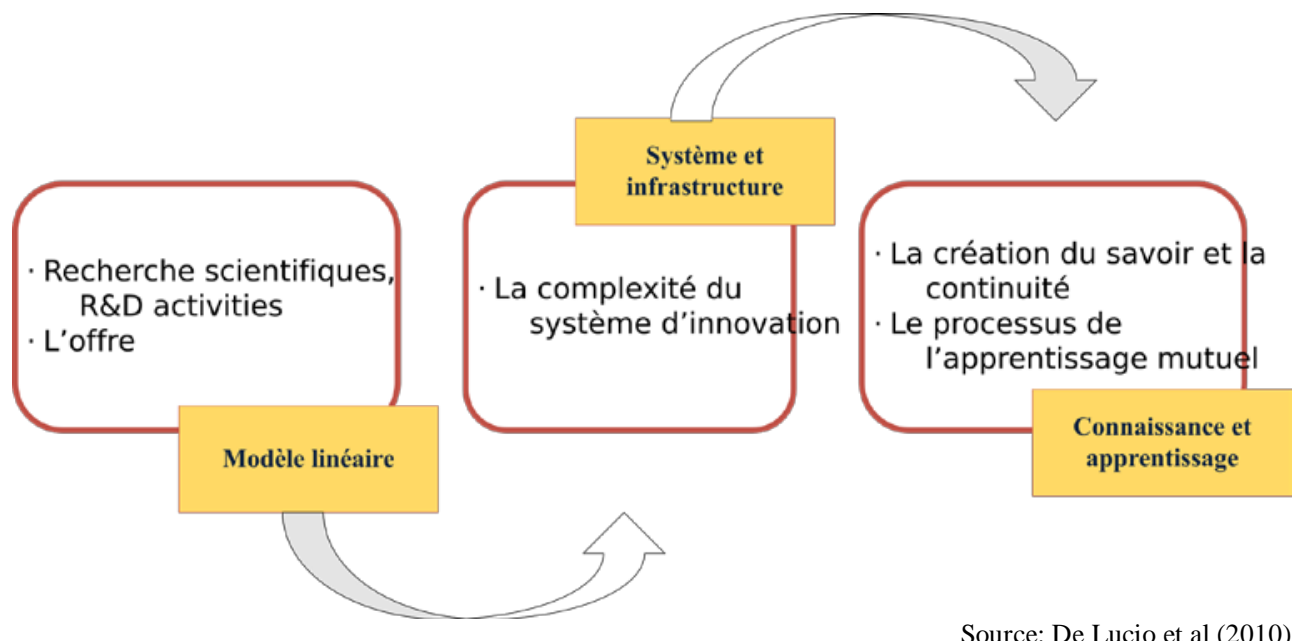

Figure 2.17 Le modèle de génération du système d'innovation

\subsubsection{Le modèle de la danse de la politique d'innovation de Kuhlmann, Shapira, et Smits (IPT)}

Kuhlmann et al. (2010) expliquent le modèle à travers la matrice de la dance de la politique d'innovation que constituent : la théorie, la politique et la pratique de l'innovation. La théorie se focalise sur l'apprentissage entre les différents acteurs qui constituent le système.

Le modèle est illustré à travers la matrice Innovation, Politique et Théorie (IPT). Car les chercheurs apprennent de la réalité des entreprises et proposent de nouvelles théories. Aussi, ils apprennent de la politique afin de participer dans le consulting. Idem pour les entrepreneurs, ils apprennent des politiques imposées et s'adaptent. En outre, les politiciens apprennent de leurs collaborations avec les chercheurs et des débats entre les acteurs afin de réajuster leur politique en faveur du développement (tableau ci-deous). 
Chapitre 2 L'état de l'art sur l'innovation, système d'innovation et politique d'innovation

Tableau 1.1 La matrice IPT d'apprentissage par interaction

\begin{tabular}{|c|c|c|c|}
\hline & Pratique d'innovation & Théorie & Politique \\
\hline $\begin{array}{l}\text { Pratique d'innovation } \\
\text { (p. Ex. Logement } \\
\text { durable) }\end{array}$ & & $\begin{array}{l}\mathrm{I} \rightarrow \mathrm{T} \\
\text { Apprendre en cherchant (par } \\
\text { exemple, les chercheurs décou- } \\
\text { vrent les relations entre utilisa- } \\
\text { teurs et producteurs à partir } \\
\text { d'expériences réelles en matière } \\
\text { de logement durable). } \\
\text { Apprendre en interagissant } \\
\text { (par exemple, les chercheurs } \\
\text { utilisent les expériences d'ac- } \\
\text { teurs comme données empi- } \\
\text { riques pour la recherche pro- } \\
\text { duite par les utilisateurs) }\end{array}$ & $\begin{array}{l}\text { I } \rightarrow \text { P } \\
\text { Apprendre en utilisant (par } \\
\text { exemple, les décideurs tirent } \\
\text { des évaluations de l'impact de } \\
\text { leurs politiques) } \\
\text { Apprendre en interagissant } \\
\text { (les décideurs, par exemple, } \\
\text { tirent des leçons de l'impact de } \\
\text { leurs politiques en s'adressant } \\
\text { aux acteurs sur le terrain) }\end{array}$ \\
\hline $\begin{array}{l}\text { Politique (par exemple, } \\
\text { politiques d'innovation } \\
\text { environnementale) }\end{array}$ & $\begin{array}{l}\mathrm{P} \rightarrow \mathrm{I} \\
\text { Apprendre en utilisant (par exemple, } \\
\text { les entrepreneurs apprennent en } \\
\text { utilisant des mesures politiques) }\end{array}$ & $\begin{array}{l}\mathrm{P} \rightarrow \mathrm{T} \\
\text { Apprendre en cherchant (par } \\
\text { exemple, les chercheurs ap- } \\
\text { prennent les relations entre } \\
\text { l'usage et le producteur grâce } \\
\text { aux politiques (axées sur les } \\
\text { politiques en matière de loge- } \\
\text { ment durable)). } \\
\text { Apprendre en interagissant } \\
\text { (par exemple, les chercheurs } \\
\text { utilisent les expériences des } \\
\text { décideurs politiques comme } \\
\text { données empiriques pour la } \\
\text { recherche } \\
\text { producteurs) utilisateurs- }\end{array}$ & \\
\hline $\begin{array}{l}\text { Théorie (par exemple, } \\
\text { interaction utilisateur- } \\
\text { producteur) }\end{array}$ & $\begin{array}{l}\mathrm{T} \rightarrow \mathrm{I} \\
\text { Apprentissage formel (par exemple, } \\
\text { les entrepreneurs tirent des théories } \\
\text { sur l'innovation utilisateur-producteur } \\
\text { et modifient leur cadre mental, leur } \\
\text { utilisation conceptuelle). } \\
\text { Apprendre en interagissant (par } \\
\text { exemple, les chercheurs agissent en } \\
\text { tant que consultants pour les entrepre- } \\
\text { neurs) }\end{array}$ & & $\begin{array}{l}\mathrm{T} \rightarrow \mathrm{P} \\
\text { Apprentissage formel (Les } \\
\text { décideurs, par exemple, tirent } \\
\text { des enseignements des théories } \\
\text { sur l'innovation utilisateur- } \\
\text { producteur et modifient leur } \\
\text { cadre mental, leur utilisation } \\
\text { conceptuelle) } \\
\text { Apprendre en interagissant } \\
\text { (par exemple, les chercheurs } \\
\text { agissent en tant que consultants } \\
\text { pour les décideurs }\end{array}$ \\
\hline
\end{tabular}

Source: Kuhlmann et al (2010, p. 8)

\subsubsection{Les modèles des instruments dans la politique d'innovation de Borrás et Edquist}

Dans leur modèle Borrás et Edquist (2013) expliquent la relation entre les instruments politiques et l'activité du système d'innovation. Ils supposent qu'il est important de 
bien choisir les instruments politiques afin d'assurer le bon fonctionnement du système d'innovation, car cette approche permet de faire resortir les défaillances du système.

En conséquence, Ils se basent sur les travaux de Bemelmans-Videc et al. (2011) pour identifier les types d'instruments politiques. Ils distinguent trois catégories d'instrument : règlement sous le nom bâton «The stick», les subventions sous le nom de la carotte «The carrot» et finalement les compagnes d'information sous le nom de «The sermon» (figure ci-desous). En l'occurrence ils démontrent que dans chaque catégorie existent des politiques qui peuvent être avantageuses et d'autres pouvant se présenter comme des barrières au développement au sein des entreprises.

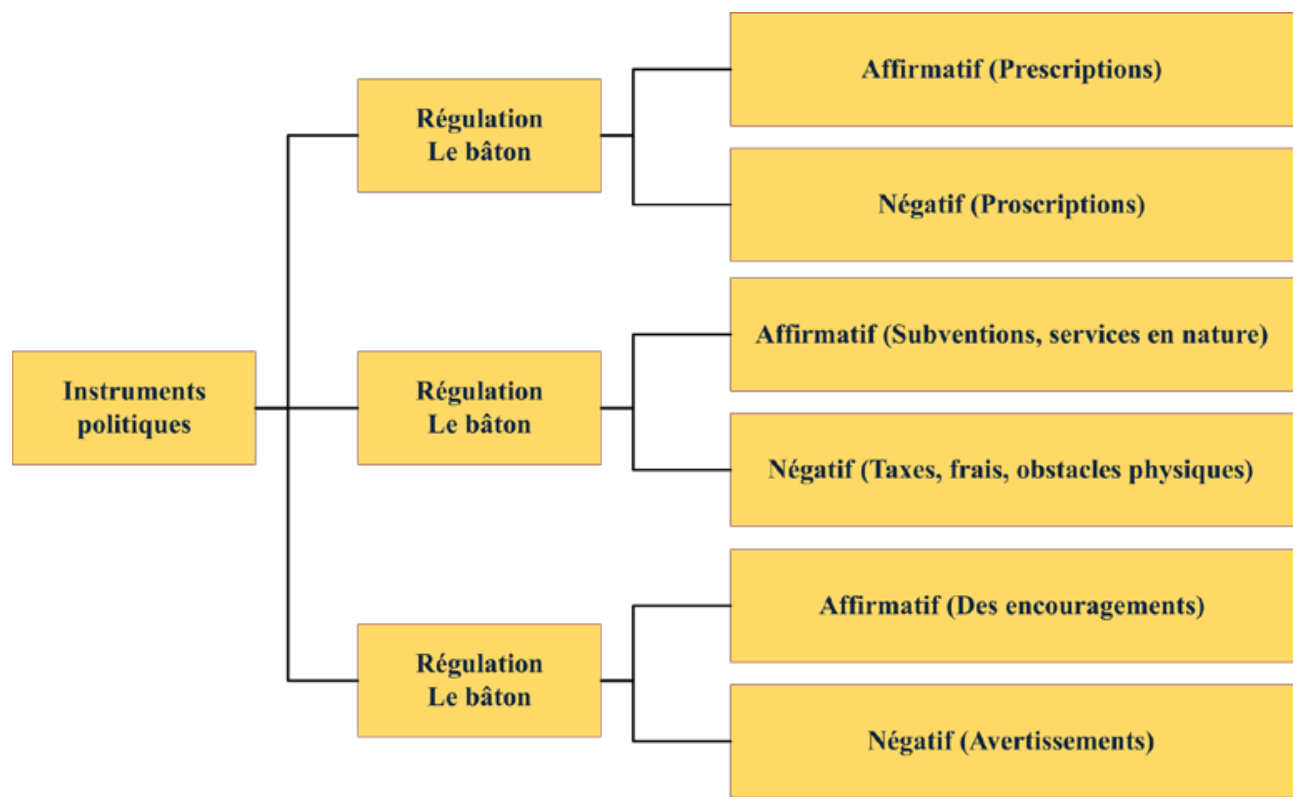

Source: Bemelmans-Videc et al. (2010, p. 250)

Figure 2.18 Type d'instruments politiques

En admettant que les instruments politiques sont spécifiques et qu'ils s'adaptent aux exigences et à l'environnement où elles se trouvent, Borrás et Edquist (2013) proposent un exemple d'instruments politiques qu'ils catégorisent aussi en trois parties (Figure ci desous)::

- Régulation, tout ce qui concerne les lois comme la propriété intellectuelle et les règlementations.

- Le transfère économique qui concerne les subventions permettant le transfert des connaissances comme les universités, le support à la recherche scientifique. 
Chapitre 2 L'état de l'art sur l'innovation, système d'innovation et politique d'innovation

- Finalement, les instruments soft représentant tout ce qui concerne la volonté de collaboration et de support administratif politique vis-à-vis de l'entreprise (Borrás \& Edquist, 2013).

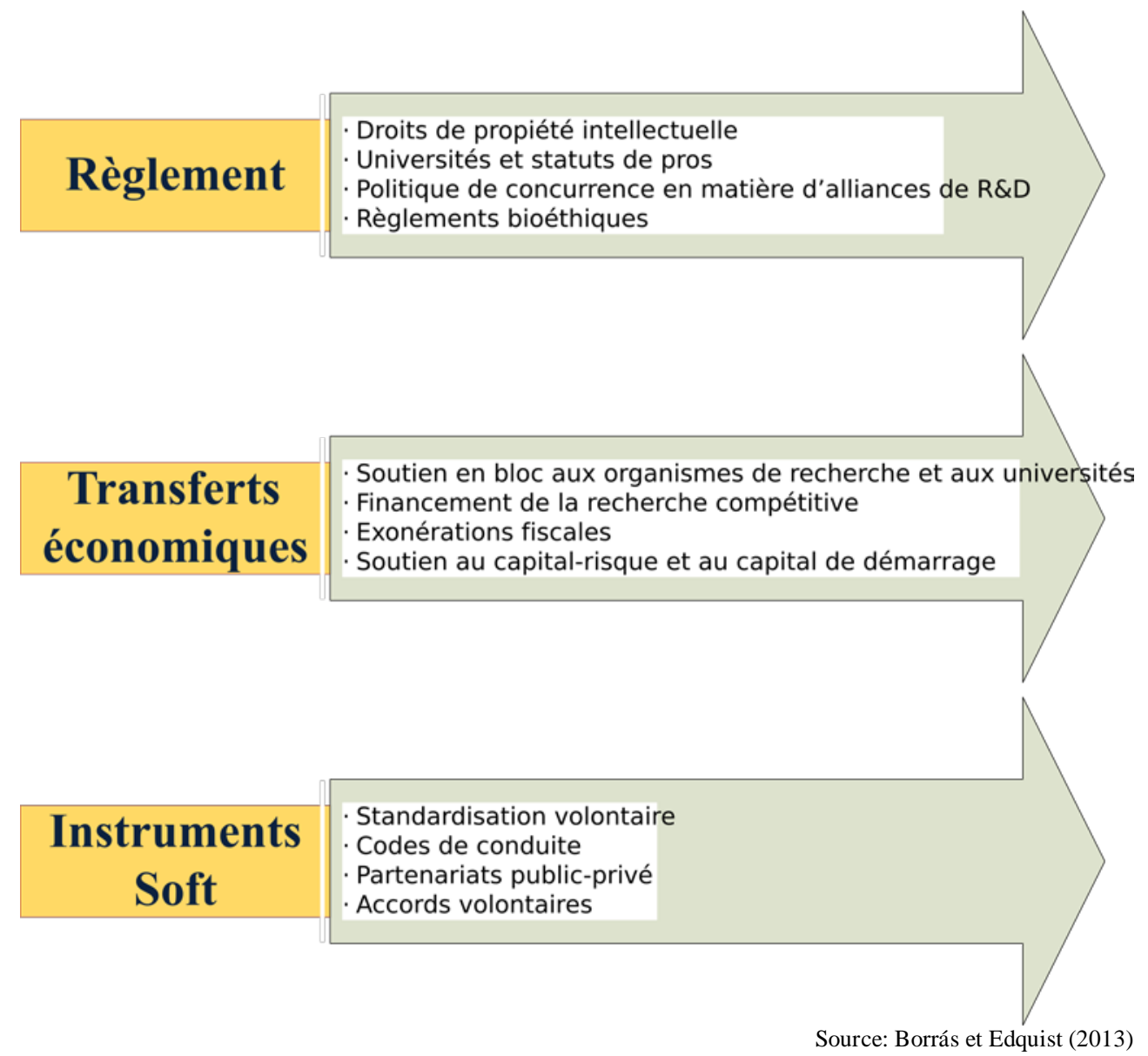

Figure 7 Principaux types d'instruments politiques

Cependant, ils se basent sur les types d'activité du système d'innovation à partir des travaux de Edquist (2011) afin de démontrer l'interaction entre le choix des instruments politiques et son impact sur la promotion de l'innovation au sein du système. A travers l'identification des activités du système d'innovation par Edquist (2011), une liste est constituée à partir de la vision linéaire du développement de l'innovation «knowledge input» au processus d'innovation. Cette liste est interprétée du coté de la demande et de l'offre aussi (Charles Edquist, 2006) : 
- L'apport des connaissances au processus d'innovation (Charles Edquist, 2006)

o Les résultats de la $\mathrm{R} \& \mathrm{D}$ (la création de nouvelles connaissances)

o Le renforcement des compétences à travers l'apprentissage individuel en ce qui concerne la formation et l'apprentissage organisationnel, l'apprentissage à travers l'expérience.

- Activités du côté de la demande (Charles Edquist, 2006)

o L'introduction de nouveaux produits au marché.

o Amélioration de la qualité des produits à l'exigence de la demande.

- Fourniture des composants (Charles Edquist, 2006)

o Créer et modifier les organisations nécessaires au développement de nouveaux domaines d'innovation. Comme l'appui aux activités d'entreprenariat et la création d'entreprises et organismes de recherche.

o La mise en réseau avec différentes organisations afin de promouvoir l'interactivité et l'apprentissage.

o La création et le changement organisationnel en fournissant des incitations à l'innovation, comme le changement dans les lois des brevets, les lois fiscales, et les normes culturelles.

- Services de soutien aux entreprises innovantes (Charles Edquist, 2006)

o Activités d'incubation telles que l'accès aux installations et le soutien administratif pour les efforts d'innovation.

o Financement de processus d'innovation et d'autres activités susceptibles de faciliter la commercialisation des connaissances et leur adoption.

o Fourniture de services de conseil pertinents pour les processus d'innovation, par exemple le transfert de technologie, l'information commerciale et les conseils juridiques.

\subsubsection{Le modèle de la mobilisation de la politique de Peck et Theodore}

Peck et Theodore (2010) suggèrent que la politique est en mutation. Elle est originale et spécifique aux espaces où elle est exploitée. La politique suit une ligne de transformation depuis son adaptation à l'exploitation. L'intérêt de la mobilisation de la politique est de comprendre comment la politique prend sa forme exploitable de l'adaptation à la modélisation de la politique. Dans ce sens Peck et Theodore (2010) explore cette vision à partir des caractéristiques de la politique d'innovation. 
Chapitre 2 L'état de l'art sur l'innovation, système d'innovation et politique d'innovation

\subsubsection{Conclusion}

En fin de compte, la politique participe au développement de la nation. Les pouvoirs publics élaborent une politique á travers un processus qui est la planification, la conceptualisation, la modèlisation et la diffusion à travers des instruments. Ces instruments sont des outils de support, d'aide au dèveloppment. En consèquences, L'intèret de la politique d'innovation est d'encourager et promouvoir l'innovation. Selon Edquist (2011), la politique d'innovation rencontre des problèmes quant à la dégradation de la performance du système national d'innovation ou de sa faiblesse. Le système d'innovation peut être simple ou complexe, tout d'epend des flux et des composantes qui le constituent. La relation entre les acteurs peut ètre verticale ou horizontale. Le système d'innovation n'est pas homogène car il peut y exister des défaillances part rapport aux contraintes de distance par exemple. La simplicité et la complexité du système d'innovation se rapportent au flux et gènèration des connaissances (figure ci desous).

\section{Interaction entre les acteurs}

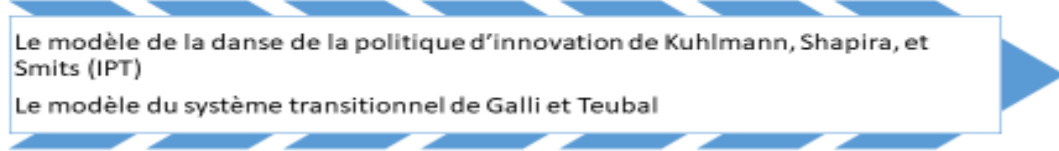

\section{La generation d'innovation au sein des enterprises}
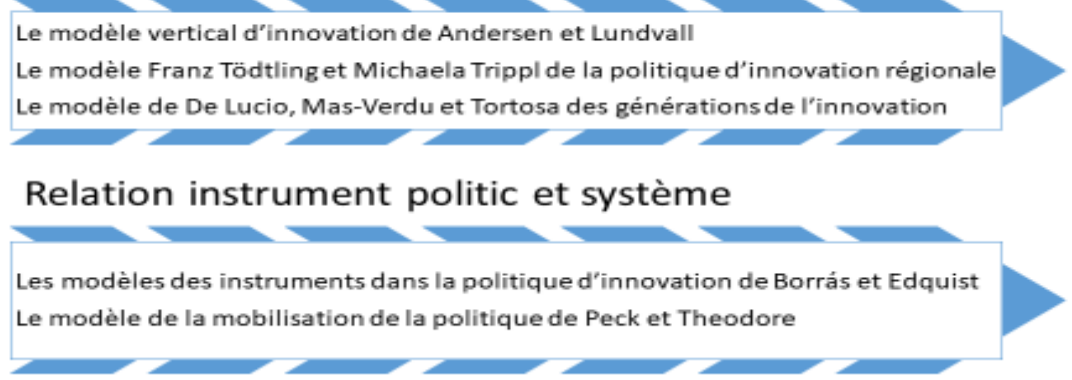

Figure 2.20 Les modes de la politique d'innovation et système d'innovation

Car chaque acteur apprend de l'interaction. Son implication à la prise de décision permet le réajustement et la correction des politiques déja entreprises. La bonne prise de dècision conduit à l'élaboration des instruments au dèveloppment des entreprises avec une grande efficacitè. Finalement, la politique peut avoir comme origine les expèriences des pays du nord mais son application aux pays du sud doit correspondre á la situation et aux besoins de chacun. A cet effet, nous consacrons la section suivante à la politique et au contexte d'innovation dans l'industrie pharmaceutique algérienne. 
Nous metons dans cette section en exergue les caractèristiques de l'industrie pharmaceutique en Algerie, puis nous montrons la répartition des entreprises pharmaceutiques en Algérie. Nous schématisons enfin la trajectoire de la politique pharmaceutique industrielle en Algerie en sélectionant tous les documents officiels qui sont aparus jusqu’à présent. Ce travail met en lumière les dispositifs mis en place afin de promouvoir l'innovation dans ce secteur.

\subsubsection{L'industrie pharmaceutique en Algérie et politique}

\subsubsection{L'industrie pharmaceutique en Algérie}

Le secteur pharmaceutique a connu une évolution importante depuis l'indépendance, vu le développement du niveau de vie, les investissementss et les améliorations dans le domaine de la santé.

L'Algérie, pays de l'Afrique du nord situé sur la mer méditerranée, a une superficie totale de $2381740 \mathrm{~km}^{2}$ avec un littoral de $998 \mathrm{~km}$ de long et où le climat est agréable toute l'année. Le produit intérieur brut (PIB) ${ }^{3}$ par an est de 150.81 billions $€$ pour l'année 2017. Les exportations s'élèvent à 29,344.1 M € par an par contre les importations sont 44,250.7 M €. En l'occurrence le taux d'inflation déclaré est d'environ $6.4 \%$ par an et le taux de chômage estimé à 10.0 \% (Source; 20184). En effet la situation économique en Algérie a beaucoup progressé depuis l'indépendance conduisant à l'amélioration appréciable du niveau de vie des individus. Les figures 21 et 22 montrent cette évolution en matière de revenu et d'échanges. Cependant, ces dernières années, l'Algérie soufre de la dévaluation de la monnaie (le Dinard Algérien) par rapport aux devises étrangères. Cela est dû à la baisse du prix du baril du pétrole et du gaz depuis 2005. En effet l'exportation des hydrocarbures représente la resource essentielle et la plus importante dans l'économie algérienne.

\footnotetext{
${ }^{3}$ Le produit intérieur brut (PIB): Est une mesure monétaire de la valeur marchande de tous les biens et services finaux, produits sur une période donnée, souvent annuelle ou trimestrielle. Les estimations du PIB nominal sont utilisées pour déterminer la performance économique d'un pays ou d'une région et pour effectuer des comparaisons internationales.

${ }^{4}$ Source: https://www.djazairess.com/
} 
Chapitre 2 L'état de l'art sur l'innovation, système d'innovation et politique d'innovation

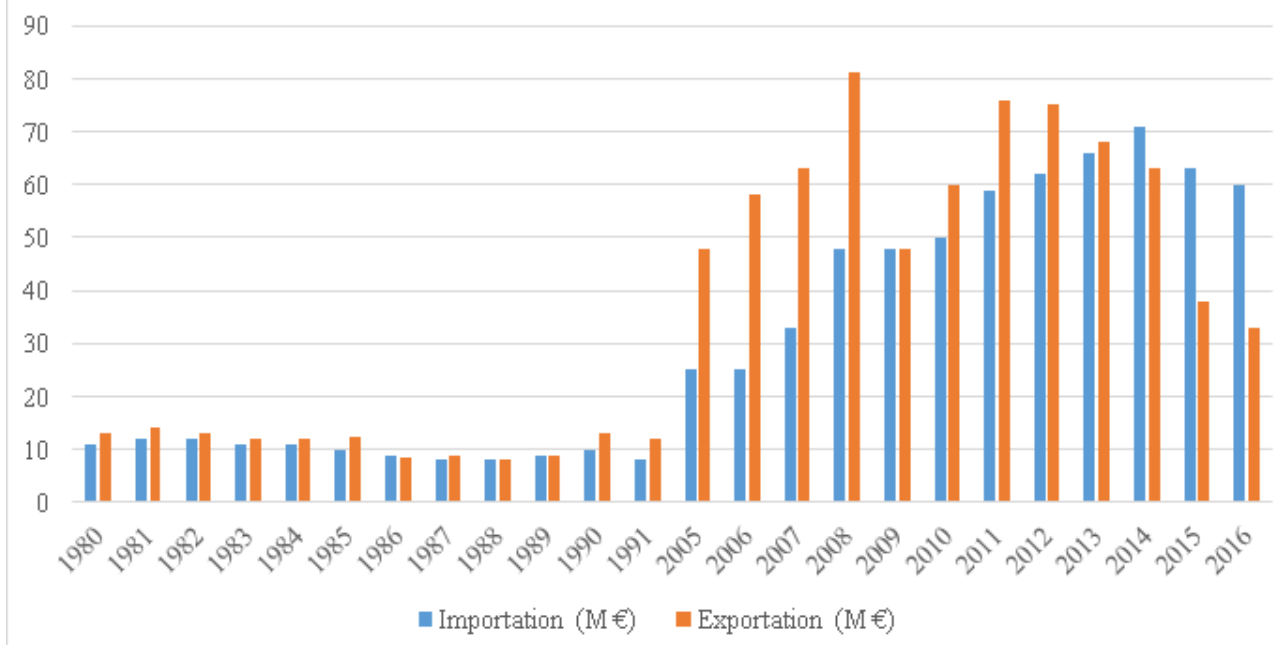

Source: https://www.worlddata.info

Figure 8 Evolution des importations et des exportations en Algérie par année

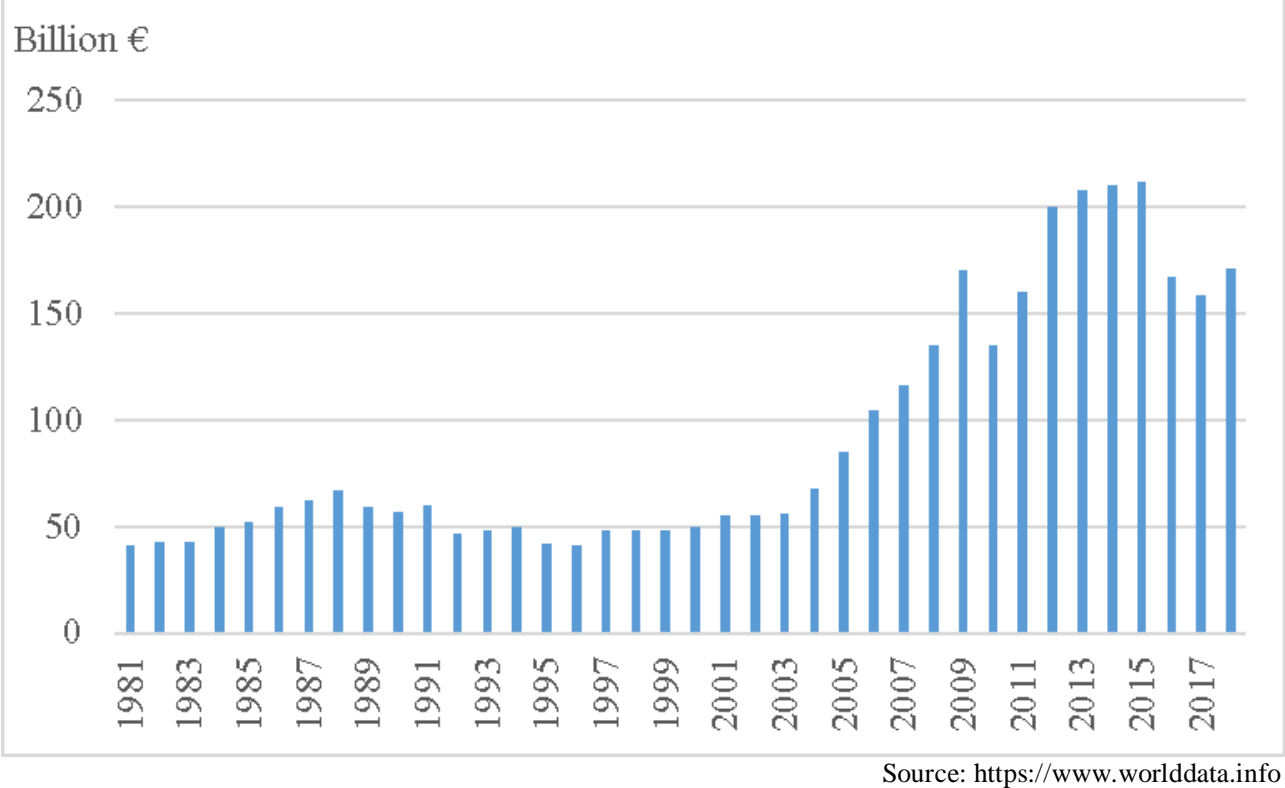

Figure 9 L'évolution du produit intérieur brut (PIB) en Algérie 
En ce qui concerne le domaine de la santé en Algérie. La forte croissance démographique observée depuis l'indépendance (les figures ci desous) a conduit en fil des ans à l'augmentation de la demande sur les médicaments et a poussé à des investissements dans l'infrastructure et dans le domaine de santé. En $2013{ }^{5}$ les statistiques sur l'infrastructure dans le service sanitaire était d'un lit pour 419 habitants soit 184 hôpitaux représentant 31000 lits, 13 CHU (Centres Hospitalo-Universitaires) pour une capacité de 16500 lits, 21 EHS (Etablissements Hospitaliers Spécialisés) d'une capacité de 5469 lits, 455 polycliniques publiques avec 3078 lits, 1123 centres de santé, 3876 salles de soins et 706 centres médico-sociaux. Contre 1.76 lit pour 100 habitants, soit 77245 lits en 2015. Le nombre de médecins par habitants est passé de 1060 en 1996 à 1118 en $2015^{6}$. Ces chiffres montrent les efforts sanitaires en matière d'investissement dans les infrastructures, la formation et les recrutements.

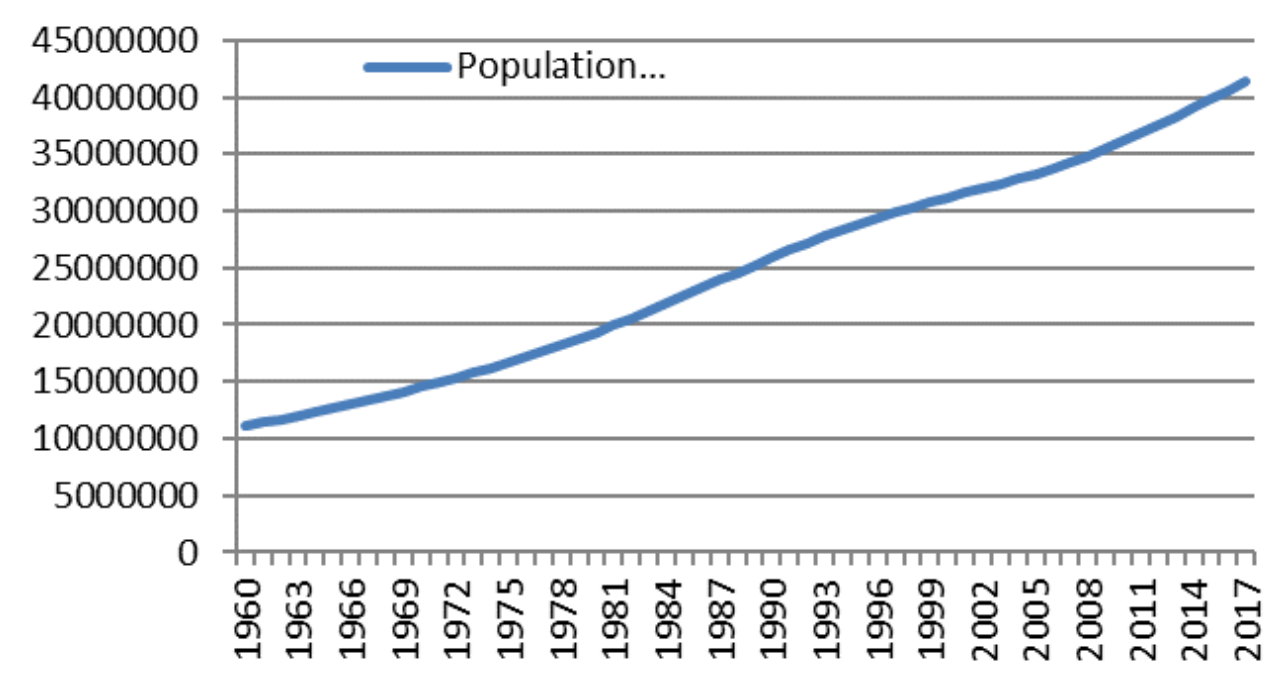

Source: https://data.worldbank.org

Figure 2.23 L'évolution de la population Algérienne par année

\footnotetext{
${ }^{5}$ Source: http://apps.who.int/medicinedocs/fr/

${ }^{6}$ Source: http://www.sante.gov.dz/
} 
Chapitre 2 L'état de l'art sur l'innovation, système d'innovation et politique d'innovation

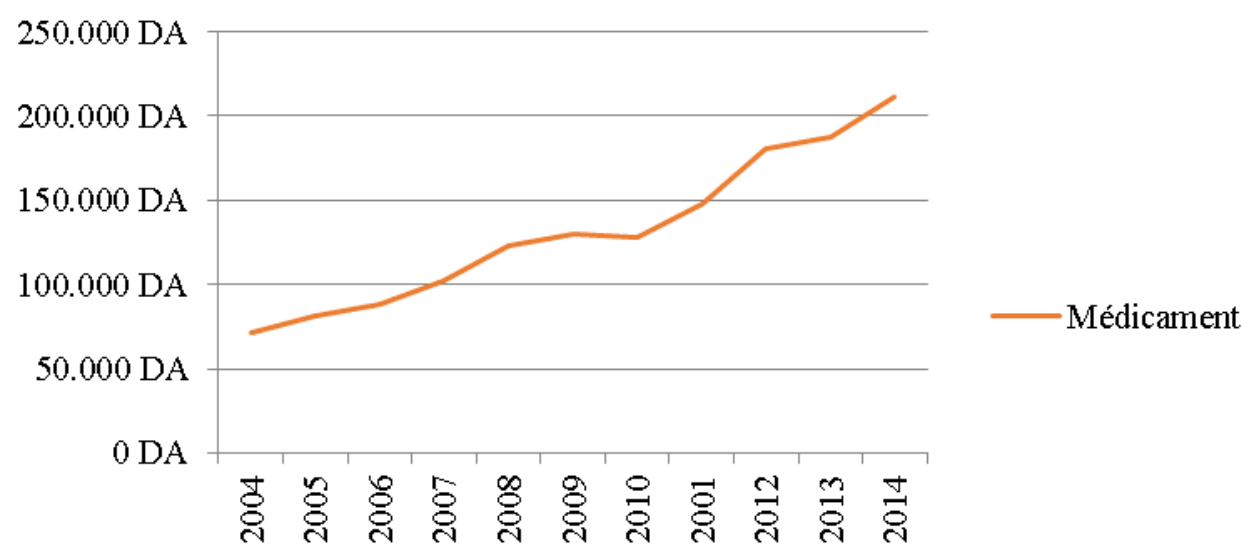

Source: http://www.ons.dz

Figure 2.24 L'évolution des importations des médicaments par année par unité en Millions de

DA

La figure 2.24 montre l'évolution des importations des médicaments par unité : En Millions de DA. On voit d'après les figures 2.23 et 2.24 que la forte demande des médicaments est reliée à l'augmentation massive de la population. Les pays qui participent à la fourniture des médicaments (Figure 2.25) sont : la France avec le plus grand pourcentage de l'importation totale soit 52\%, vient ensuite l'Allemagne avec $18 \%$. L’importation de médicaments est devenue indispensable vu les faibles capacités de productions du pays et l'extension du marché pharmaceutique. Aussi le choix politique du pays dépend des relations bilatérales avec ce dernier et ainsi de l'appartenance des médicaments aux grands laboratoires qui se trouvent dans ce pays. 


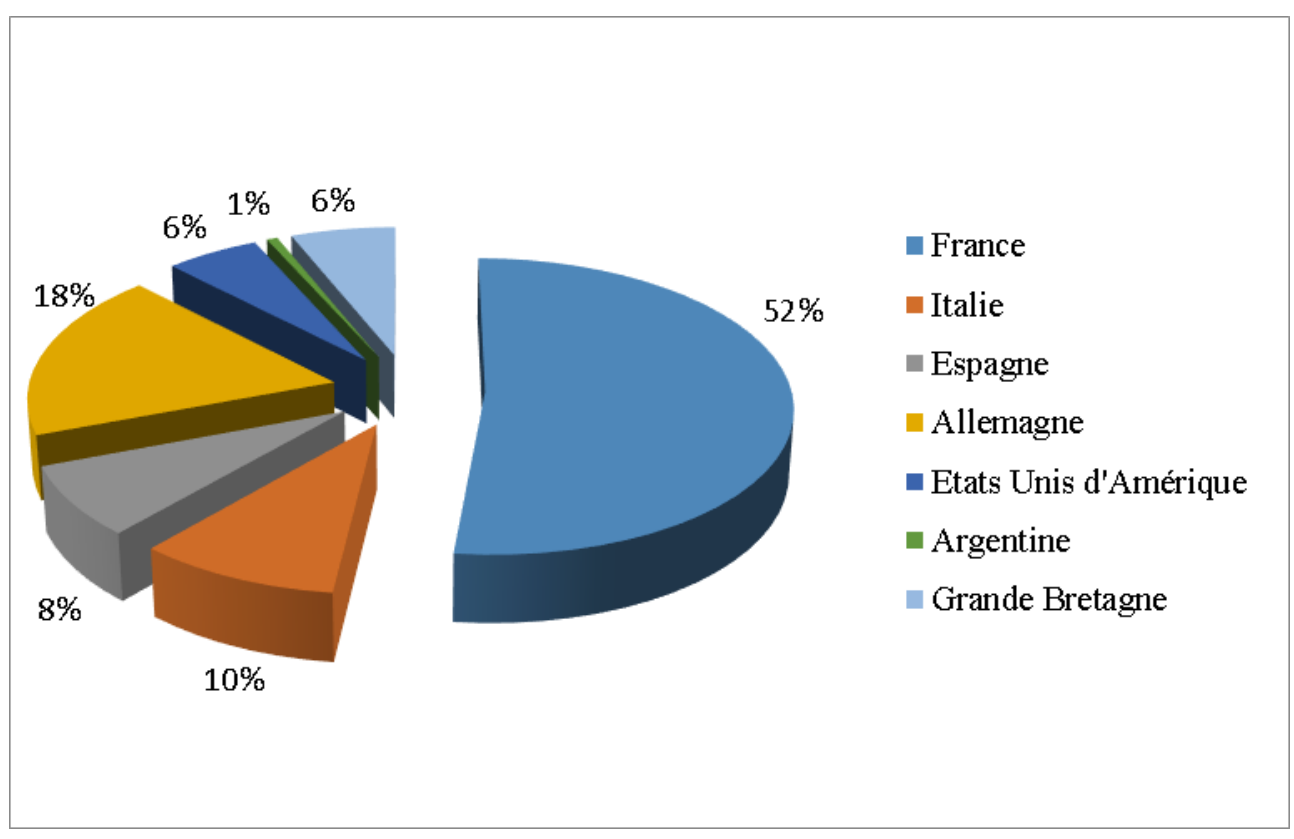

Source: http://www.ons.dz

Figure 10 La part des pays étrangers des importations des médicaments en Algérie par unité

\subsubsection{La politique pharmaceutique en Algérie}

Le secteur pharmaceutique en Algérie est en effet en extension car une politique de promotion de la production locale ainsi qu'une politique de substitution à l'exportation des hydrocarbures a été instaurée suite à la déficience du budget national à cause des chutes du prix du baril de pétrole depuis 2004. Les entreprises pharmaceutiques et parapharmaceutiques sont incluses dans la catégorie des entreprises en relation avec la chimie. Cette catégorie représente $4 \%$ du nombre total d'entreprises du territoire national, soit 35\% représente la part des entreprises pharmaceutiques et parapharmaceutiques (source La chambre de commerce Algérienne ${ }^{7}$ ). Il existe 140 entreprises parapharmaceutiques dont 71 sont des entreprises publiques et privée de production des médicaments (figure ci desous). Plus de $57 \%$ de ces entreprises sont des petites et moyennes entreprises (CHOUAL, 2016). Les entreprises sont reparties dans le terri-

\footnotetext{
${ }^{7}$ Source: https://www.commerce.gov.dz/
} 
toire national dans 25 wilayas $^{8}$ sur un total de 48 afin d'assurer la bonne distribution des médicaments. La plus grande concentration des entreprises pharmaceutiques se trouve au centre du pays, à Alger, à l'Est Constantine et Annaba et à l'ouest Oran. La part du marché local de la production des médicaments est de 131376982 046, 21 DA soit 1255994092 en €, celle des dispositifs médicaux est de 5939683 838,87 da soit $52048463 €$. La fabrication locale est estimée à $45 \%$ de la demande contre $55 \%$ pour l'importation. Le groupe SAIDAL ${ }^{9}$ couvre $10 \%$ de la production nationale (source ministère de la sante Algérie du $2015^{10}$ ), (Figure ci desous).

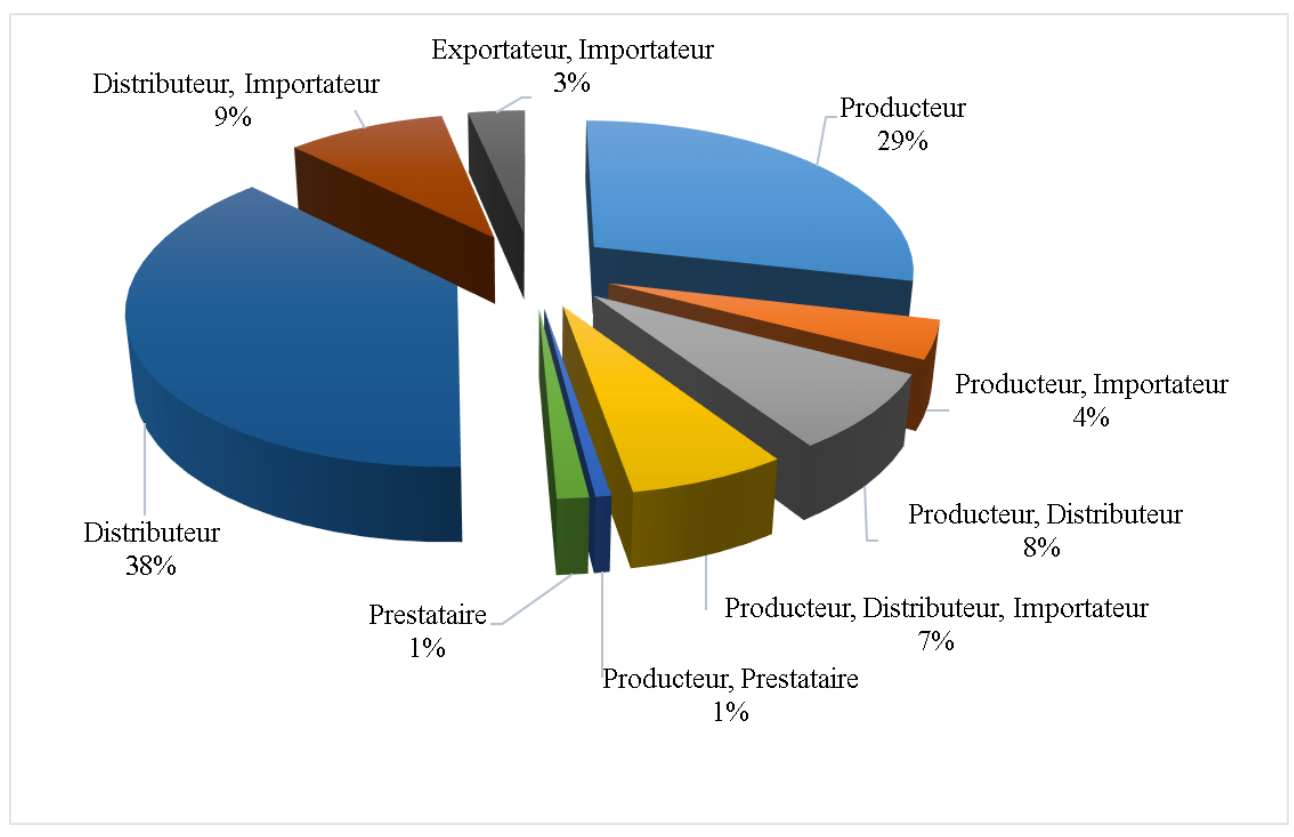

Source: https://www.commerce.gov.dz

Figure 11 Le nombre d'entreprises pharmaceutiques par activité

\footnotetext{
${ }^{8}$ Equivalant selon les États au département, à la région, au canton ou à la province

${ }^{9}$ Un groupe dont $80 \%$ capital de l'Etas et 20 sont des actionnaires est considérer parmi les leaders de la production pharmaceutique algérienne en Algérie.

10 Source: http://www.sante.gov.dz/
} 


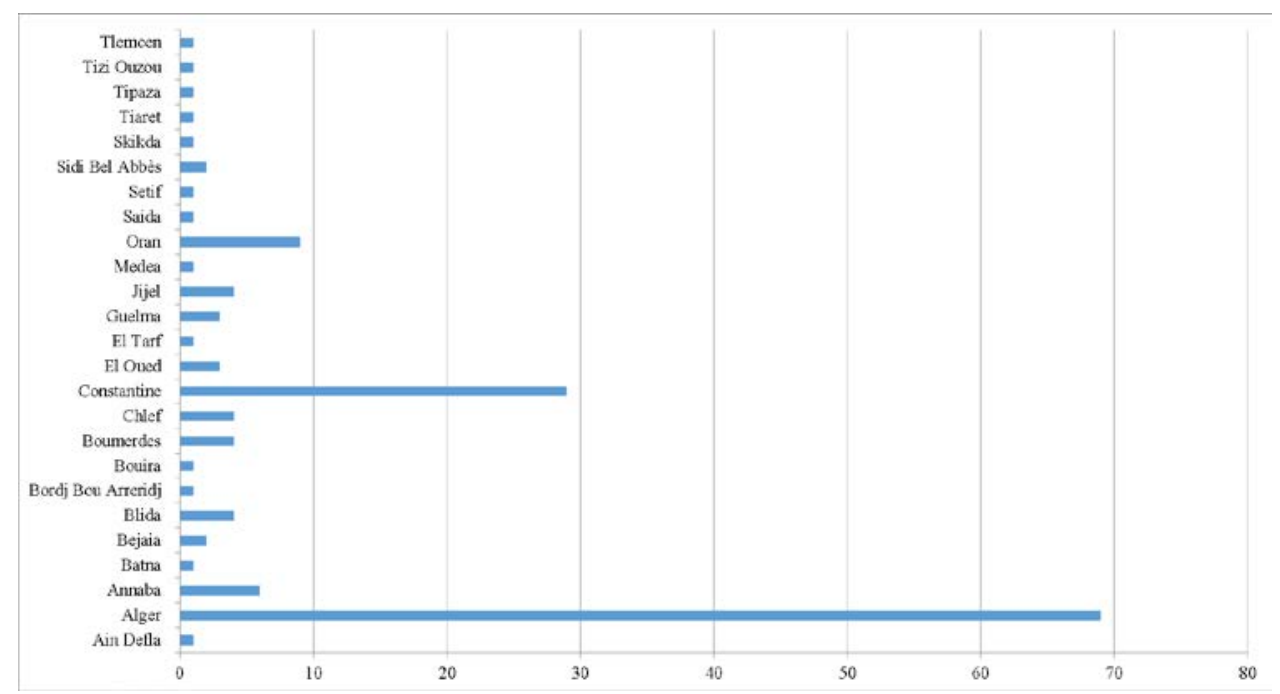

Source: https://www.commerce.gov.dz

Figure 12 La répartition des entreprises par wilaya

2.4.7.3 L'évolution de la politique d'innovation du secteur pharmaceutique en Algérie

Le secteur pharmaceutique a connu plusieurs politiques de structuration depuis l'indépendance jusqu'à la libération totale. La politique était caractérisée par instauration et réajustement depuis 1969 (Figure 2.28).

La naissance de la pharmacie en Algérie est apparue avec la colonisation française de 1830 à 1960 (Bonnemain, 2009). De nombreux chercheurs et pharmaciens français se sont installés en Algérie pour le développement et la recherche dans le domaine minéral vu la richesse naturelle du sol algérien. Cette recherche a permis aussi de transférer les connaissances du nord vers le sud. Il existait à l'époque une concentration importante de militaires ; un besoin donc de médecins et de pharmaciens s'est présenté. Ce qui a conduit à une politique de formation dans le domaine de la santé ; c'est ainsi que s'est créée la pharmacie centrale Algérienne. L’industrie pharmaceutique était basée sur une seule entreprise BIOTIC qui a été créée en 1952.

De 1962 à 1969: le secteur pharmaceutique dépendait du ministère de la santé; il a été géré par l'administration du secteur de la santé. Car cette période a connu un rétrécissement (Santé, 1997) dans le domaine pharmaceutique par la fermeture des laboratoires de recherche et des entreprises après le depart des coopérants français qui détenaient le savoir. Après l'indépendance les entreprises pharmaceutiques étaient à l'abandon (Djeflat \& Hamadi, 2016). 
La période de 1969 à 1976 a connu une structuration de l'institution pharmaceutique ; en 1969 le gouvernement a proclamé le monopole de l'importation des produits pharmaceutiques sous l'ordonnance 69-14 du $1969^{11}$. Cette mesure a été suivie par une préparation à l'indépendance de la production par un décret qui donne une certaine marge d'autonomie à l'institution pharmaceutique en Algérie (qui dépendait du ministère de la santé), comme la commercialisation, la règlementation des produits pharmaceutiques et l'exercice des laboratoires ${ }^{12}$. Finalement en 1976 on est passé vers une réorganisation de l'institution de la pharmacie ${ }^{13}$.

De 1977 jusqu'à 1983, la pharmacie a obtenu une indépendance vis à vis de l'administration du ministère de la santé car en 1977 a lieu l'approbation du statut de l'entreprise de la pharmacie centrale Algérienne (PCA) ${ }^{14}$. Cette approbation est suivie par la création des entreprises d'approvisionnement en produits pharmaceutiques à l'Ouest du pays à Oran ${ }^{15}$, à l'Est à Constantine ${ }^{16}$ sous le nom de «ENCOPHARM» et au centre à Alger $^{17}$ «ENAPHARM» et l'unité économique «ENAPHAR» . Le développement du secteur pharmaceutique privé en Algérie a connu un grand essort. Cette politique est basée sur la substitution des entreprises privées à caractère publique à l'administration pharmaceutique algérienne en maitre de la distribution et la diffusion.

En 1985 le gouvernement a émis une loi (85-05) concernant la protection et la promotion de la santé. Car la période de 1985 jusqu’à 1990 a connu une ouverture du marché vers l'extérieur ${ }^{18}$; la pharmacie centrale algérienne a réparti la fonction d'importation entre les institutions d'approvisionnement. Aussi durant cette période, a eu lieu la création d'unités de recherches ${ }^{19}$ par l'entreprise publique SAIDAL dans le domaine des médicaments et des techniques pharmaceutiques. Cette dernière a influencé le prix et la

\footnotetext{
11 Source: JO N² 29 du 04 Avril 1969, Page 226

12 Source: JO N 61 du 31 Juillet 1973, Page 671/ JO N 33 du 23 Avril 1974, Page 357/ JO Nº 49 du 20 Juin 1975, Page 552/ JO $\mathrm{N}^{\circ} 1$ du 02 Janvier 1977, Page 8/ JO $\mathrm{N}^{\circ} 1$ du 02 Janvier 1977, Page 5

13 JO N 1 du 02 Janvier 1977, Page 2

14 JO Nº 9 du 30 Janvier 1977, Page 143/ JO Nº 17 du 27 Avril 1982, Page 561

15 JO N 17 du 27 Avril 1982, Page 568

16 JO N 17 du 27 Avril 1982, Page 566

${ }^{17}$ Source: JO N 17 du 27 Avril 1982, Page 564/ JO Nº 24 du 07 Juin 1983, Page 1091

18 JO Nº 32 du 31 Juillet 1985, Page 733/ JO Nº 9 du 02 Mars 1988, Page 277

19 JO N 52 du 23 Décembre 1987, Page 1354
} 
régulation du marché ${ }^{20}$ des produits pharmaceutiques donc les prix à l'achat et la production ont vu une réévaluation.

De 1991 à 1992 le gouvernement a soutenu une politique de réajustement de la chaine de production; des réévaluations des conditions et les modalités à la production et à l'importation ${ }^{21}$ ont été enregistrées, car cette période était affectée par une crise économique causée par la chute du prix des hydrocarbures. En l'occurrence en $1993^{22}$ la politique était appliquée aux conditions de fonctionnement des laboratoires de production. Puis en 1994 une nécessité vers la régulation du marché s'est imposée vu l'extension de la production et le volume d'importation des médicaments.

De 1994 à 1995 une politique de réajustement du système pharmaceutique ${ }^{23}$ Algérienne a été menée. Elle portait sur l'instauration des organisations de contrôle et des systèmes de vérification de la qualité des produits pharmaceutiques, en créant un processus qui exige du produit importé ou fabriqué localement de respecter des conditions de norme de qualité et de réajustement des prix. À cet effet un arrêté est apparu (133) ${ }^{24}$ exigeant une fixation du prix de référence de remboursement de la caisse nationale de sécurité sociale. En 1996 la politique focalisait sur la proposition de techniques de production ${ }^{25}$ d'enregistrement des médicaments. La politique de la promotion du produit local était à ce moment là très forte. L'arrêté 29 du $1996{ }^{26}$ fixe une liste des médicaments importés de l'étranger ${ }^{27}$ car la demande sur certains médicaments était satisfaite par la production locale. Dans cette année la politique était focalisée sur la

$20 \mathrm{JO} \mathrm{N}^{\circ} 11$ du 14 Mars 1990, Page 342/ JO N 11 du 14 Mars 1990, Page 341/ JO Nº 11 du 14 Mars 1990, Page 338/ JO N 11 du 14 Mars 1990, Page 337/ JO Nº 33 du 08 Août 1990, Page 930

21 JO Nº 25 du 29 Mai 1991, Page 749/ JO Nº 25 du 29 Mai 1991, Page 748/ JO Nº 25 du 29 Mai 1991, Page 746/ JO N 53 du 30 Octobre 1991, Page 1719/ JO Nº 9 du 05 Février 1992, Page 210

22 JO Nº 32 du 16 Mai 1993, Page 7/ JO N 32 du 16 Mai 1993, Page 6/ JO N 47 du 18 Juillet 1993, Page 28/ JO N 44 du 07 Juillet 1993, Page 8/ JO N 44 du 07 Juillet 1993, Page 7/ JO N 41 du 20 Juin 1993, Page 6/ JO N 59 du 22 Septembre 1993, Page 8/ JO N 59 du 22 Septembre 1993, Page 6/ JO Nº 15 du 20 Mars 1994, Page 8

23 JO Nº 9 du 16 Février 1994, Page 5/ JO Nº 63 du 05 Octobre 1994, Page 8/

${ }^{24}$ Arrêté Interministériel N133 du 21/12/1995

25 JO Nº 84 du 29 Décembre 1996, Page 18/

${ }^{26}$ Arrêté n 6 du 06/02/1996/ Arrêt N 8 du 08/02/1996 / Arrêté N 7.9.14.13.30 du 8/02/1996. 09/02/1996 /21/02/1996. 21/02/1996. 06/05/1996.

${ }^{27}$ Arrêté n 29 du 06/05/1996 
création des réseaux entre les organisations et les institutions ${ }^{28}$ qui constituent le secteur pharmaceutique.

En 1997, une politique plus dédiée à certains produits spécifiques comme la classification et la diffusion de l'information médicale ${ }^{29}$ et les modalités de contrôle. En l’occurrence l'année 1998 suite au décret 98-68 est créée l'agence INAPI de la propriété intellectuelle Algérienne qui a permis à l'entreprise SAIDAL de recherche en médicaments de poser 15 brevets dont 12 sont développés avec des partenaires étrangers, soit 3 brevets en interne, entre 2005 et $2010^{30}$. Cette dernière a boosté l'accroissement et l'accumulation des connaissances en matière de biochimie et de pharmacie industrielle.

En 1999, le gouvernement à réajusté la politique de fonctionnement de la commission centrale d'agrément à la production des médicaments en vu de la promotion de la production locale. A cet effet la politique du gouvernement n'a pas fait de différences entre les entreprises étrangères et nationales car la première entreprise SAIDAL a commencé déjà par collaborer avec les entreprises leaders de la pharmacie afin de transférer le savoir vers l'industrialisation pharmaceutique. Les principaux investisseurs étrangers en Algérie sont: Le français Sanofi Aventis avec la part de marché de 320 millions \$ en 2009, Hikma pharma avec 164863013 dollars, GSK avec une part de marche de 141958937 dollars, Novartis avec 129138999 dollars, Pfizer avec 111 323448 dollars). Le danois Novo Nordisk avec 85264536 dollars, le français Roche diagnostics Astrazeneca et finalement l'américain MSD avec une part du marché de 85 264536 dollars $^{31}$.

En 2001, la loi portant sur l'exonération des droits et taxes des produits chimiques et organiques ${ }^{32}$ destinés à la production pharmaceutique a été appliquée. Cette dernière a été modifiée en $2006^{33}$ dans le cadre de la promotion de la production locale, les couvertures des besoins du marché local et la substitution à l'importation. En l'occurrence,

\footnotetext{
28 Décret 96-68 du 27/01/1996 / Décret exécutif 96-67 27/01/1996 / Décret exécutif 96-355du 19/10/1996 / JO N 51 du 06 Août 1997, Page 14

29 Arrêté N6 du 20/01/1997 / Arrêté N18 du 13/02/1997/ ArrêtéN38 du 06/04/1997 / Arrêté N37 Du 08/04/1997 / Arrêté N40 du 21/04/1997 / JO Nº 70 du 26 Octobre 1997, Page 12

${ }^{30}$ Rapport sectorielle du ministère de l'industrie et des mines 2011

31 Rapport sectorielle du ministère de l’industrie et des mines 2011

32 JO N 60 du 17 Octobre 2001, Page 13

33 JO N 32 du 17 Mai 2006, Page 4
} 
la tombée de plusieurs brevets de la propriété privée dans le domaine publique après 20 ans du dépôt, a ouvert une grande marge au marché Algérien pour acquérir les génériques en investissant dans la production de nouveaux produits pharmaceutiques. Cette démarche créa une rude concurrence entre les entreprises locales de productions et de commercialisation puisque le choix d'investir dépend du cout de la technologie et des conditions de productions ainsi que de la rentabilité. La politique Algérienne est basée sur la disponibilité des médicaments auprès des citoyens ; la sécurité sociale Algérienne joue un rôle important dans l'équilibre du niveau de vie et le bien-être du citoyen algérien. Donc la mise à jour et le réajustement dans les modalités et les conditions de remboursement ${ }^{34}$ des médicaments (figure 2.29) est un enjeu pour les entreprises pharmaceutiques en Algérie.

34 JO N$^{\circ} 2$ du 15 Janvier 2006, Page 19 / JO N 42 du 15 Juin 2005, Page 29/ JO Nº 74 du 21 Novembre 2004, Page 16 


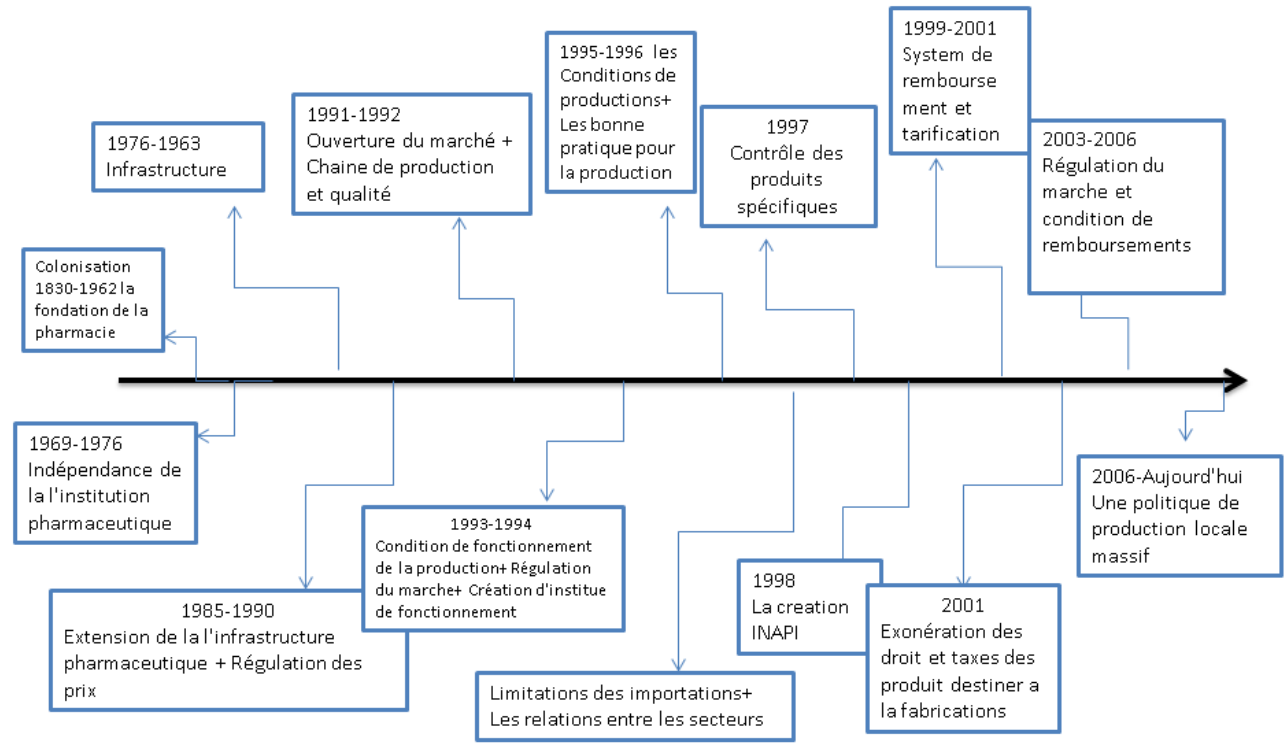

Figure 2.28 L’évolution de la politique de l'industrie pharmaceutique en Algérie

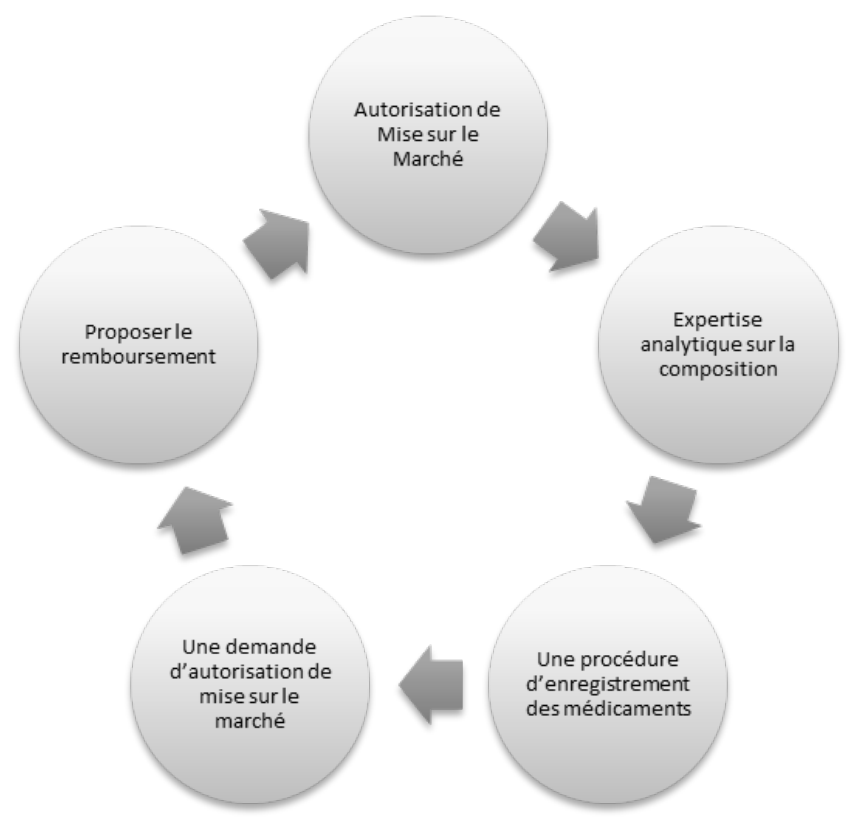

Figure 2.29 La mise sur le marché et l'enregistrement des médicaments 


\subsubsection{Conclusion}

L'objectif de cette section était de comprendre le comportement du système politique d'innovation, l'interaction entre les acteurs, le rôle des institutions, l'impact des instruments politiques et les orientations politiques industrielles au sein des entreprises. Plus précisément, l'étude a été caractérisée par une description du secteur pharmaceutique Algérien, par une analyse de l'industrie pharmaceutique et finalement par une analyse du système et la politique entreprise depuis l'indépendance à ce jour. Nos résultats confirment la relation entre institutions et politiques industrielles. En revanche l'étude ne soutient pas la relation entre système d'innovation et instruments politiques.

En effet la politique dans le secteur pharmaceutique Algérien est basée sur l'infrastructure, l'extension, les conditions de fonctionnement de la production, la création d'instituts, les incitatifs destinés à la fabrication, la régulation du marché, une politique de production locale et finalement la centralisation des décisions politiques et administratives. Des lors, l'institution algérienne joue un rôle premièrement coopératif en matière de régularisation des prix et remboursement sur les médicaments. Deuxièmement, elles jouent un rôle incitatif à travers les exonérations des droits et taxes des produits destinés à la fabrication. Tandis que, la concentration des entreprises est affectée par la concentration des administrations supposées collaborer. Par ailleurs, la politique industrielle Algérienne à aucun moment n’a traité la question de la promotion de l'innovation ou soutenu le développement des produits pharmaceutiques spécifiques. Deuxièmement, le ministère de la santé exige un protocole de qualité des produits . Troisièmement, la politique de la production locale est bénéfique dans le sens ou les entreprises Algériennes investissent plus sur de nouveaux produits. C'est pourquoi, l'entreprise Algérienne a toujours besoin du transfert technologique étranger et de matière première. Dans la mesure où l'industrie pharmaceutique algérienne est en extension, elle a plus besoins de subventions et de collaborations dans le domaine du développement et l'adaptation de la technologie. Plus le gouvernement investit sur les laboratoires de recherche et la qualification des employés plus cela aidera les entreprises à se développer rapidement et à acquérir le savoir et le savoir-faire.

Après l'analyse des caractéristiques de l'industrie pharmaceutique et les orientations politiques vis-à-vis du secteur, notre préoccupation principale est de vérifier les points suivants : le bon fonctionnement de l'entreprise par rapport aux délais de traitement des dossiers et réclamations, si les administrations publiques manquent d'outils et de qualifications pour faire face à l'extension du marché des médicaments en Algérie et finalement si ces entreprises n'ont pas les compétences pour se lancer dans la production à haute technologie ni pour développer des produits à des prix concurrentiels. Nous allons découvrir l'état des entreprises dans la prochaine section qui est consacrée à l'étude descriptive des réponses des responsables des entreprises pharmaceutiques. 


\section{Chapitre 3 Cadre conceptuel de la recherche}


Evaluation de la politique d'innovation sur un échantillon d'entreprises algériennes 
Ce chapitre est le rèsultat de rèflection après lecture des revues de l'iteratures sur l'etat conceptuel de l'innovation, sur le système d'innovation ainsi que sur la politique d'innovation. Car, avant d'entamer une politique d'innovation nous devons nous assurer si toutes les composantes du système d'innovation sont connectées. Du fait l'évaluation de la politique est systématique. D'après la section précédente, la politique d'innovation dans les pays en dèveloppment diffère d'un pays à l'autre. A cet effet, nous allons proposer un modèle thèorique qui permet de systématiser la prise de décision ainsi que l'evaluation de la politique dans ces pays là. Il est basé sur des ètudes antérieures sur la politique d'ìnnovation dans les pays en dèveloppment, et a pour objectif d'extraire la politique appropriée au développement des entreprises.

\subsection{Proposition du modèle et élaboration d'hypothèses}

Le modèle théorique proposé comporte cinq dimensions : les agents d'innovation, les instruments politiques publics, les activités d'innovation des entreprises, les barrières que les entreprises trouvent dans leur intention et leur activité d'innovation, et enfin les résultats de l'innovation. Toutes ces dimensions sont interconnectées.

Tout au long de cette section, la relation de chacune de ces dimensions avec les facteurs prédominants est présentée. Ces facteurs sont tirés de diverses sources. D'une part, la littérature existante sur les résultats du travail de politique d'innovation dans les pays en développement sont analysés (A. D. Andersen, 2012b, 2012a; Capriati, 2017; Dantas \& Bell, 2011; Dutrénit \& Sutz, 2014, p. 388; Iizuka \& Katz, 2012; Katz, 2015; Morris et al., 2012; Sesay et al., 2018). Et, d'autre part, l'enquête sur l'innovation de l'Union européenne (ECI) de 2014 est prise en compte. Ainsi, grâce à l'étude approfondie de chacune de ces dimensions, il est désormais possible de comprendre l'impact de chaque composante sur la prise de décision politique et les résultats de l'innovation. $\mathrm{La}$ figure ci deous montre le modèle théorique proposé. 


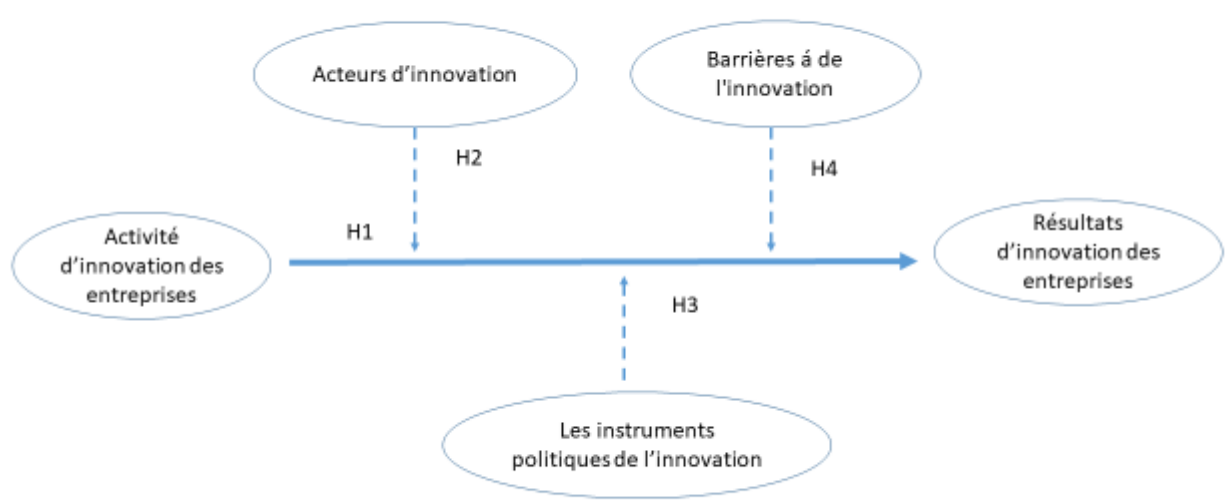

Figure 3.1 Modèle théorique (basé sur divers auteurs cités)

Selon ce modèle, les résultats des entreprises en matière d'innovation sont la conséquence de leurs activités d'innovation. Cette relation sera modérée par les politiques d'innovation du pays, ainsi que par les acteurs ou agents impliqués dans le système d'innovation.

Les quatre facteurs ou éléments qui interviennent ont été analysés par la littérature académique comme nous le commentons ci-dessous : les activités d'innovation, les agents d'innovation, les instruments politiques publics d'innovation et les résultats de l'innovation.

\subsection{Développement d'hypothèses}

La politique d'innovation est engendrée par l'instauration des instruments politiques. Une politique basée sur l'instauration d'instruments politiques dans le cadre de la promotion de l'innovation, est-elle considérée comme un moteur de changement et de décollage pour les pays en développement ? Dutrenit et Puchet, dans «l'analyse de l'évolution institutionnelle et la politique d'innovation au Mexique» (Dutrénit \& Puchet, 2017) proposent trois instruments: les incitations à la recherche et développement (R \& D) pour les entreprises, la création d'un fond pour le renforcement des liens entre université et l'entreprise et ainsi instaurer une vision large de la politique aux décideurs politiques pendant la préparation des budgets. Est-ce que cette politique est applicable dans d'autres pays ? Est-ce que ces instruments garantissent le succès du bon fonctionnement du système d'innovation? 
En l'occurrence, le système d'innovation, tel qu'il est dans les pays en développement, s'inspire des pays avancés. Ce phénomène est surnommé la réification du système national d'innovation (Delvenne \& Thoreau, 2017, p. 41). La réification est due à la simplification du système de son origine de conception. Quant à la définition du SNI (Système National d'Innovation), elle est supposée inclure : la compréhension de la nature, du rythme et de la direction du processus du changement technologique, de ses économies d'une part, et également inclure l'identification des acteurs déterminants du système, en admettant que le système national d'innovation conduit aux prescriptions politiques appropriées.

A cet effet, des critiques ont été appréhendées. Les enseignements tirés des politiques des pays industrialisés ne fournissent pas nécessairement des modèles de politiques d'innovation utiles pour les pays en développement (Djeflat, 2010). Rennkamp (2017, p. 87) affirme que le cadre des systèmes nationaux d'innovation ne permet pas d'analyser les politiques d'innovation dans des sociétés inégales, puisque il n'explique pas les processus de négociation entre les acteurs impliqués dans le processus politique. En outre, Pierre Delvenne et Thoreau (2017, p. 51) rajoutent l'aspect des approches des SNI qui ont tendance à ne pas tenir suffisamment compte du contexte sociopolitique et du contexte mondial. Marcelle (2017, p. 72) implémente la problématique plus en profondeur. Le système d'innovation dans les pays en développement doit prêter attention aux micro-fondements de la dynamique de l'innovation ou à la dynamique de variation contextuelle et à la spécificité de l'innovation.

Tandis que certains chercheurs proposent une autre conception de la politique d'innovation par l'innovation inclusive dite aussi croissance inclusive (George et al., 2012; Adrian Smith et al., 2014). La croissance est inclusive lorsqu'elle permet à tous les membres d'une société de participer et de contribuer au processus de croissance sur un même pied d'égalité quelles que soient leurs circonstances individuelles. Ainsi, en utilisant le cadre chaînes de valeur mondiales, l'interaction entre les acheteurs mondiaux et les producteurs locaux dans les pays moins développés peut générer des activités d'apprentissage et d'innovation (Orozco, 2017, p. 298). Cette nouvelle approche de la politique d'innovation remet en question l'adéquation de la politique d'innovation ainsi que le système national d'innovation. Il s'est avéré que la politique d'innovation dans les pays en développement est construite sur la base d'apprentissage. Mieux on arrive à comprendre le flux et la circulation du savoir et du savoir-faire, mieux on arrive à comprendre les défaillances du système d'innovation.

Notre modèle est basé sur la systématisation de la prise de dècision, sur l'évaluation de la politique et la compréhension du système politique d'innovation. Le processus se compose de quatre dimensions qui sont connectées entre elles avec trois inputs et un output (Figure ci desous).

Cette étude s'inspire des travaux faits dans le cadre de la compréhension de l'efficacité de la politique d'innovation: Premièrement, l'action publique doit se concentrer sur 
l'adaptabilité du système d'innovation avec l'objectif global (Charles Edquist, 2011) de créer un cadre national ou régional propice à l'adaptabilité des entreprises et à l'exploitation efficace des opportunités offertes par le monde extérieur. Cependant, la politique se transforme au cours de son adaptation, d'une vision globale à la vision territoriale ou régionale (Peck \& Theodore, 2010; Tödtling \& Trippl, 2005a). Deuxièmement, l'Instrument Politique. Il est le résultat de l'action publique, dans la mesure ou les décideurs politiques, après l'élaboration d'une politique ils finiront par l'élaboration d'instruments qui permettent d'intervenir dans le processus de développement que ce soit en matière de loi comme la propriété intellectuelle qui est un support à l'innovation et aussi un support administratif collaboratif avec les acteurs du système d'innovation (Borrás \& Edquist, 2013). A cette raison, le choix des instruments, varie selon la politique d'innovation instaurée (de Lucio et al., 2010). Troisièmement, l'activité innovatrice (Borrás \& Edquist, 2013; Charles Edquist, 2006) au sein des institutions. Cette dernière est traduite par la coïncidence des objectifs de l'innovation au sein des entreprises avec les instruments politiques, car les instruments politiques doivent être adaptés aux exigences des institutions en matière d'innovation. Finalement, l'interaction des acteurs est interprétée par l'apprentissage (B.-A. Lundvall, 1985) à travers l'interaction entre les acteurs. Sous prétexte que, les acteurs apprennent à travers leur expérience au moment de leur collaboration ou leur interaction avec d'autres acteurs (Kuhlmann et al., 2010). En conséquence les chercheurs apprennent à travers leurs recherches et leurs expérimentations, les entrepreneurs et les instituts apprennent de la politique instaurée et des résultats de recherche et les décideurs politiques apprennent aussi à travers des collaborations et évaluation de la politique publique. Notre modèle se compose de quatre dimensions : trois inputs les acteurs de l'innovation, les instruments politiques, les activités de l'innovation qui sont connectés entre eux ; et un output les résultats de l'innovation. 


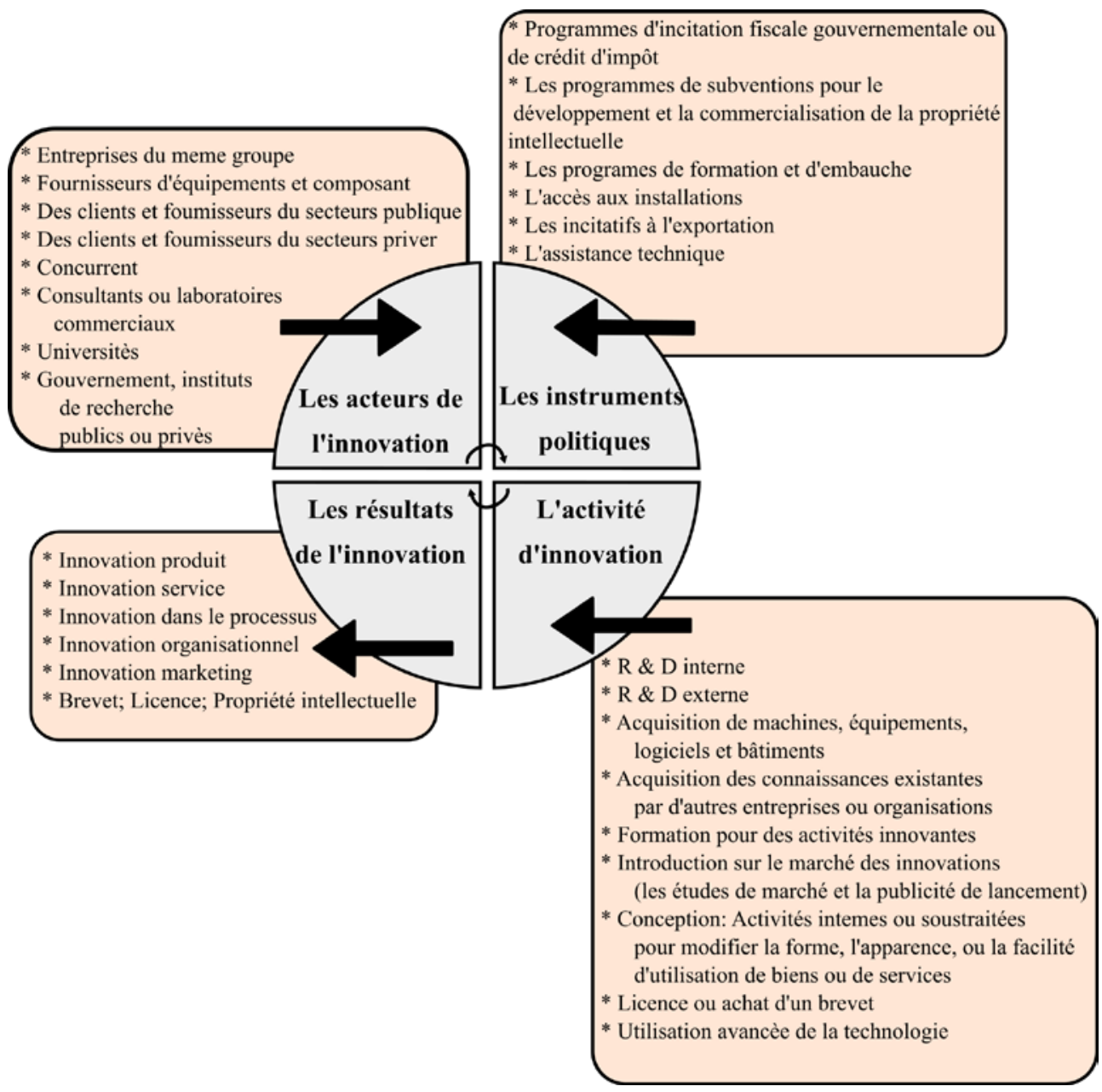

Figure 3.2 Le modèl théorique proposé

\subsubsection{Activités d'innovation}

Les activités d'innovation impliquent un engagement de l'entreprise à promouvoir l'innovation et la performance. En fait, les entreprises investissent dans l'activité d'innovation dans le développement de produits et aussi dans l'acquisition de connaissances internes ou externes (Becker \& Dietz, 2004; C. Criscuolo, Haskel, \& Slaughter, 2010; Parida, Westerberg, \& Frishammar, 2012) .

À cette fin, les activités des entreprises doivent être orientées. Galende et de la Fuente (2003) ont déterminé dans quelle mesure les ressources et les facteurs internes d'une 
entreprise expliquent le processus d'innovation et les activités développées pour atteindre l'objectif de l'innovation.

L'activité de l'innovation est liée avec les résultats d'innovations. En effet, Frank et al., (2016) montrent comment les entreprises brésiliennes dans les secteurs industriels contribuent à l'innovation. Leurs résultats montrent que l'industrie brésilienne a tendance à adopter deux stratégies d'innovation ; l'innovation orientée vers le marché et l'acquisition de technologie. A cet effet l'activité de l'entreprise doit être orientée. Galende et de la Fuente (2003) ont déterminé dans quelle mesure les ressources internes et les facteurs d'une entreprise expliquent le processus d'innovation et les activités développées pour atteindre l'objectif d'innovation.

Les institutions affectent l'innovation dans les entreprises. Pezeshkan et al., (2016) ont fait une étude d'après 47 économies de pays en développement. Ils s'aperçoivent que les institutions complémentaires et substituables facilitent l'innovation dans les entreprises des pays en développement. En conséquence le gouvernement doit penser à l'instauration d'institutions qui réduisent les barrières à l'innovation. Les institutions nationales encouragent l'innovation dans les entreprises.

La performance de l'entreprise est en relation avec le niveau du transfert des connaissances; en effet la problématique de l'innovation à une relation avec la présence des connaissances. Tey et Idris (2012) examinent l'effet médiateur du transfert de connaissances sur la relation entre l'adéquation culturelle et les performances d'innovation. Les résultats de leur étude, affirment que l'adéquation culturelle organisationnelle et l'adéquation nationale sont des déterminants positifs du transfert de connaissances. Ces résultats suggèrent que l'effet du transfert de connaissances sur l'innovation comportementale est atténué par le facteur des valeurs de gestion interne. En outre, Da Silveira (2001) suggère que la diffusion de l'innovation dépend de trois aspects qui sont l'économie et le gouvernement, les stratégies technologiques des entreprises et la gestion de l'innovation.

L'activité d'innovation dans les pays en développement diffère de l'activité d'innovation dans les pays avancés. Effectivement, Deniozos (1994) explique que la demande des entreprises vers la technologie dans les pays en développement est élevée par rapport à celle des pays développés. Cette demande est accompagnée par la capacité d'absorption de cette technologie.

L'activité d'innovation exige une coordination entre les acteurs. D'après Gabriela Dutrénit et Puchet (2017) les coalitions entre les acteurs du système sont déterminées par leurs croyances et leurs ressources. En conséquence, la politique d'innovation est affectée par la rareté, donc une vue alternative est propagée entre les acteurs du systèmes (Srinivas \& Sutz, 2008b). A cet effet, s'il existe une faible demande vers les connaissances, cela affecte l'effet de la politique axée sur la promotion de la diffusion des connaissances dans les entreprises (Arocena \& Sutz, 2017, p. 403). Donc l'activité 
d'innovation est accompagnée par une politique de la promotion des connaissances dans le cas où il existe une volonté pour innover.

Selon le CIS, les activités d'innovation comprennent l'acquisition de machines, d'équipements, de bâtiments, de logiciels et de licences, des travaux d'ingénierie et de développement, des études de faisabilité, la conception, la formation, la R\& $D$ et le marketing, lorsqu'ils sont spécifiquement destinés à développer et / ou mettre en œuvre un produit ou un procédé innovant. Cela inclut également tous les types de R \& D consistant en des activités de recherche et développement visant à créer de nouvelles connaissances ou à résoudre des problèmes scientifiques ou techniques. Cette dimension est inspirée du questionnaire «The Community Innovation Survey» (CIS) du 2014 et les travaux qui ont été faits sur la relation entre l'activité d'innovation et les résultats d'innovations. Les activités d'innovations dans notre étude incluent les activités suivantes :

a) Le besoin d'investir sur la $\mathbf{R} \& \mathbf{D}$ interne est caractérisé par une capacité et des compétences internes pour mener la recherche avec les moyens de l'entreprise. Baumol, d'après son étude (2004) sur les entreprises asiatiques, explique que les entreprises continuent d'innover pour survivre là où il existe une forte concurrence. En outre, Intarakumnerd et al., (2012) montrent dans une études menée sur le secteur de l'automobile en Asie que l'acquisition des compétences adéquate pour le développement dans la R\&D est constituée des facteurs internes et des facteurs externes. Les facteurs internes s'identifient à l'intention stratégique, le processus d'apprentissage et la capacité d'absorption des entreprises. Tandis que les facteurs externes sont le cycle de vie de la demande et des produits, l'environnement réglementaire et politique. A cet effet, l'interaction entre les ressources externes et internes conduit à la création et au renforcement des capacités d'innovation des entreprises. Cette capacité est assugétie au rôle des universités et des instituts de recherche publics pour aider les entreprises locales à développer des capacités innovantes.

b) Le besoin des entreprises vers une $\mathbf{R} \& \mathbf{D}$ externe, Cette demande est caractérisée par l'incapacité de l'entreprise à investir dans la recherche et développement avec les compétences existantes en son sein. Comme, Cho et al.,(2015) expliquent que l'efficacité de la R\&D dépend du type d'industrie et de sa taille. En outre les interactions internationales en matière de recherche, constituées de la collaboration interpersonnelle, la collaboration institutionnelle et la collaboration au sein des multinationales, se sont multipliées en raison de la mondialisation et de la complexité technologique croissante (Stek \& van Geenhuizen, 2016). Cependant, l'impact des interactions internationales de recherche $(R \& D)$ sur la performance de l'innovation nationale dépend du secteur et du type de technologie. 
c) La nécessite de formation pour des activités innovantes est la clef du succès dans les entreprises, mais elle est couteuse. La formation doit être qualifiée et orientée vers les besoins technologiques de l'entreprises (Clemens, Graham, \& Howes, 2015). Hansen et Ockwell (2014) induisent que les techniques et les mécanismes d'apprentissage différents dans l'accumulation de capacités technologiques. Les résultats de leur recherche montrent que les entreprises qui s'appuient à la fois sur l'apprentissage par l'intermédiaire de partenaires technologiques étrangers et sur l'apprentissage interne par des expérimentations planifiées progressent mieux en termes de capacités technologiques (McCullagh \& Meyer, 1997). A cet effet, l'investissement sur la formation doit être évalué sur la base d'accumulations des capacités technologiques (B.-Å. Lundvall, 2006).

d) L'activité d'introduction sur le marché des innovations (les études de marché et la publicité de lancement), le CIS, la définit comme une activité interne ou sous-traitée pour l'introduction sur le marché des biens ou services nouveaux ou sensiblement améliorés, y compris l'étude de marché et la publicité de lancement. En l'occurrence, le IBSP du 2017 la considère comme un nouveau produit sur un de ses marchés, par exemple a introduit un nouveau, ou sensiblement amélioré, bien ou service sur l'un des marchés de cette entreprise avant ses concurrents (il peut avoir déjà été disponible sur d'autres marchés).

e) L'acquisition de machines, équipements, logiciels et bâtiments est la stratégie la plus connue dans les entreprises des pays en développement. Cette activité est menée par une adaptation de la technologie laquelle nécessite des compétences pour s'adapter. A cet effet, les politiques doivent s'impliquer pour promouvoir l'adoption d'une nouvelle technologie (Abate, Rashid, Borzaga, \& Getnet, 2016). En effet, Paunov et Rollo (2016) montrent que l'effet de l'utilisation d'internet par les industries affecte positivement la productivité moyenne de l'entreprise et ses investissements en équipements et augmente la probabilité pour que les entreprises obtiennent des certificats et des brevets de qualité. Donc l'entreprise doit être en mesure à adopter la technologie à travers l'acquisition de machines nouvelles et équipements.

f) Le besoin d'acquisition des connaissances existantes par d'autres entreprises ou organisations est rattaché à la possibilité de transfert technologique. L'augmentation du produit intérieur brut par habitant dans les pays en développement est imputable au transfert international de technologie, via des investissements directs étrangers (Di Vita, 2013). En outre, Franco et al., (2011) ont comparé les pratiques d'innovation utilisées par les filiales des multinationales dans deux grandes économies émergentes, l'Inde et le Brésil, d'intrants technologiques différentes pour l'innovation. Le système national 
d'innovation est un facteur d'appui ou de défaillance en ce qui concerne les liens de connaissances entre les filiales des multinationales et les systèmes nationaux d'innovation. En l'occurrence une recherche vers l'acquisition des connaissances doit être menée avec une stratégie de sélection et de l'efficacité du transfert des connaissances au sein de l'entreprise (Guan \& Yan, 2016). A cet effet Arocena et Sutz (2010) expliquent qu'il existe une faible demande du marché pour le savoir. Les politiques scientifiques et technologiques et d'innovation du côté de l'offre et du côté de la demande sont en dessous des attentes. Alors, les politiques doivent être complétées de manière rentable par un ensemble de politiques d'innovation conçues comme des politiques sociales, capables de répondre à une forte demande sociale et de développer des capacités innovantes endogènes.

g) Le besoin d'une sous-traitance pour la facilité d'utilisation de biens ou de services est associé avec la génération des connaissances au sein de l'entreprise. Car, Egbetokun (2015) analyse la relation entre l'apprentissage interactif et les capacités dans un échantillon d'entreprises manufacturières nigérianes. Les résultats montrent que les entreprises peuvent devenir plus capables d'innover si elles communiquent avec des connaissances générées de l'extérieur. Cependant, le choix de la manière et du moment de créer des liens ainsi l'importance des liens formels et informels affectent l'interactivité de l'apprentissage.

h) Utilisation avancée de la technologie est une activité qui demande un savoirfaire vis-à-vis de l'entreprise. A cet effet, J. J. Lee et Yoon (2015) identifient différents modèles d'apprentissage technologique des retardataires, d'après, les expériences de la Corée du Sud, de la Chine et du Brésil en matière de développement d'avions militaires. Les résultats montrent que la différence existe dans le processus d'apprentissage de la capacité technologique locale. L'adoption d'une technologie influence le processus d'apprentissage par la pratique.

i) Certaines entreprises des pays en développement optent pour l'achat d'une License ou brevet afin d'assurer le transfert technologique. Reste que le prix d'investissement varie selon la technologie du brevet ou la License en question. Etant donné que, Danzon et towse (Danzon \& Towse, 2003) a étudié dans le domaine pharmaceutique, le rapport avec le cas économique des brevets et la possibilité d'une tarification différenciée pour accroître l'accessibilité des médicaments brevetés dans les pays en développement tout en préservant les incitations à l'innovation. Cette initiative permet l'acquisition du savoir-faire et le transfert technologique dans les pays en développement.

La troisième dimension nous permet maintenant de formuler la troisième hypothèse . 
Hypothèse 1: L'activité d'innovation des entreprises influence positivement les performances d'innovation.

\subsubsection{Les acteurs d'innovation}

Les acteurs de la politique d'innovation dans notre modèle sont les agents externes. A travers ces agents, l'entreprise acquiert des connaissances, et se met en relation avec d'autres dans le but de satisfaire un besoin. En effet, Etzkowitz et Brisolla (1999, p. 298) l'ont expliqué par le renforcement institutionnel auquel les conditions suivantes s'imposent: consolidation d'un système financier favorable, vision intégrale de l'entreprise, génération d'une culture d'évaluation de la performance en examinant tous les facteurs critiques qui la déterminent tout le long de la chaîne de valeur, coordination des efforts entre les différents acteurs institutionnels, analyse de nouvelles opportunités commerciales, création de mécanismes de renforcement des capacités de la population afin de promouvoir les nouvelles entreprises, renforcement des réseaux de collaboration entre les différents acteurs pour traiter adéquatement les aspects critiques de la performance dans chaque nouvelle entreprise et finalement améliorer la capacité d'une vision stratégique.

Dans notre modèle nous nous basons sur l'enquête canadienne de 2017 «Integrated Business Statistics Program (IBSP) Survey of Innovation and Business Strategy» et du questionnaire «The Community Innovation Survey» (CIS) du 2014. Selon l'enquête du CIS, La coopération peut inclure les R\&D et les projets qui ne comprennent pas les R\&D. En ce qui concerne l'enquête canadienne de 2017 (IBSP) les collaborations sont considérées comme la coopération active. Car, la coopération active est la participation d'autres entreprises ou organisations à des activités d'innovation. Les deux partenaires n'ont pas besoin de bénéficier commercialement. Donc le choix des acteurs dans notre modèle est conçu à partir du questionnaire CIS 2014 et de l'enquête canadienne IBSP de 2017 revue par la littérature pour s’adapter aux pays en développement.

a) Une entreprise qui préfère collaborer horizontalement ou verticalement. Sous prétexte que, la politique du gouvernement peut se focaliser sur l'incitation sur la communication dans une grappe ou cluster dans un même secteur d'activité par exemple. Cette politique permet aux entreprises d'être à jour sur les techniques et d'acquérir plus de connaissances (Nishimura \& Okamuro, 2011); cette vision est verticale. La vision horizontale est en l'occurrence, le réseautage entre les entreprises du même groupe ou entre la hiérarchie dans le cadre multinational. Cette vision se trouve plus dans les grandes entreprises où les instituts de recherche sont actifs et les multinationales. On peut appliquer cette perception sur les entreprises pharmaceutiques dont l'entreprise mère détient la molécule mère du produit. En d'autres termes (Jin, Wang, \& Vanhaverbeke, 2014), le cas de la Chine appuie l'hypothèse selon laquelle les multinationales déposent le plus souvent des brevets par 
l'intermédiaire de leur siège (P. Criscuolo, 2006). Encourager cette politique assure une absorbion des capacités technologiques au sein des entreprises ?

b) L'acquisition d'un nouvel équipement, de matériaux, de composants ou de logiciels à travers des fournisseurs, est considéré enrichissante. Du fait que, l'échange avec d'autres entreprises permet d'acquérir des compétences dans l'amélioration du processus de fabrication et d'être compétitif. A cet effet, les pays en développement ne sont pas les fournisseurs de la technologie en général mais sont des adaptateurs. L'intervention du gouvernement visant à encourager une stratégie axée sur l'investissement, en mettant le gros des efforts d'investissement sur l'adoption de technologies étrangères, pourrait constituer une stratégie de développement appropriée (Herrerias \& Orts, 2013). Certaines entreprises investissent dans les actifs incorporels puisqu'ils représentent l'aspect le plus impactant sur l'innovation (Montresor \& Vezzani, 2016). Mais est-ce que toutes les entreprises en voie de développement ont la capacité d'adaptation au changement?

c) L'offre au secteur public : Les entreprises correspondent aux insuffisances que les politiques ne traitent pas suffisamment. Cela découle du manque de couverture, du manque d'appropriation par les acheteurs, de l'absence de prise en compte de l'ensemble du cycle d'acquisition et de la maîtrise de l'aversion pour le risque (Georghiou, Edler, Uyarra, \& Yeow, 2014). Est-ce l'aspect distance et écart spatiale a un impact sur la connexion entre ces entreprises?

d) La collaboration entre les concurrents : Il existe des facteurs qui favorisent la coopération interentreprises dans les pays en développement, des facteurs liés aux entrants nécessaires à la coopération, notamment «la confiance, la communication et la réciprocité», «l'engagement des principaux dirigeants», «des accords bien documentés», «des ressources coopératives suffisantes». Ces facteurs influents sur la coopération interentreprises dans le contexte chinois (D. Chen, Karami, Chen, \& Karami, 2010). D’autre part, les facteurs spécifiques à la R \& D influencent la probabilité que les entreprises soient des partenaires d'alliance attrayants. En particulier, les entreprises affichant un historique d'innovation axé sur un ou plusieurs produits sont plus susceptibles d'être des partenaires d'alliance avec des entreprises des grandes économies émergentes plus attrayants que celles qui ne le sont pas. Dans les PME biotechnologiques britanniques et allemandes (De Mattos, Burgess, \& Shaw, 2013). A cet effet, les entreprises disposant de plus de ressources, d'un meilleur partage des connaissances et d'un meilleur réseau accélèrent plus efficacement leur processus d'innovation (Xie, Wu, \& Ma, 2016)

e) La culture de collaboration vis-à-vis des consultants ou laboratoires : Dans leurs recherches, Intarakumnerd et Chaoroenporn (2013) dans le secteur de l'automobile, ont évoqué le rôle des intermédiaires dans le secteur automobile 
en Thaïlande et comment le cadre institutionnel affecte la performance des intermédiaires de l'innovation. A cet effet, les intermédiaires publics et privés devraient collaborer et avoir une compréhension mutuelle des types d'organisations afin de permettre le transfert du savoir. Ainsi, les associations industrielles en tant qu'acteurs intermédiaires clés facilitent l'échange de connaissances et le renforcement des capacités institutionnelles, en particulier dans les pays en développement où les capacités institutionnelles sont limitées (Papaioannou, Watkins, Mugwagwa, \& Kale, 2016). Comment le gouvernement peut-il promouvoir les intermédiaires dans le cas d'insuffisances institutionnelles?

f) L'université est représentée comme un moteur générateur des connaissances dans les pays en développement : Fikirkoca et Saritas (2012) décrivent le processus de prospective et stratégie politique en proposant le parc scientifique de l'Université d'Ankara. Ils discutent les facteurs de succès pour les parcs scientifiques dans trois dimensions comprenant la « complémentarité », le « réseautage» et le «positionnement stratégique». Cette dernière explore l'impact de l'activité entrepreneuriale de l'université sur la compétitivité régionale. En l'occurrence, Les résultats ont montré que les facteurs informels (par exemple, les attitudes, les modèles de rôles) ont une influence plus importante sur l'activité entrepreneuriale universitaire que les facteurs formels (par exemple, mesures de soutien, éducation et formation) (Guerrero, Urbano, \& Fayolle, 2016). A cet effet, la relation entre la recherche universitaire dans les universités et le transfert de connaissances et la relation entre le système national d'innovation et le système d'enseignement supérieur se base sur la manière avec laquelle se comporte la recherche et son influence sur le secteur économique (Kwon, 2011). La production scientifique doit être bien diffusée et orientée.

g) Les entreprises éprouvent une demande pour le développement par apport de la $R \& D$ vis-à-vis des organisations publiques et instituts de recherche publics ou privés : Tseng et Chen (2014) illustrent par une étude de cas portant sur la manière dont l'ITRI et le HSIP du parc industriel scientifique de Hsinchu (ITRI) ont aidé des PME de Taiwan à surmonter les défis de la technologie, des ressources humaines et des secteurs. La problématique de l'offre ne s'arrête pas là. La transition des flux de connaissances vers la compétence des organismes de recherche dans les pays en développement nécessite la volonté du gouvernement et l'internalisation des connaissances (Park, Jeong, Yoon, \& Lee, 2015). En général une politique de promotion de la R\&D dans les pays en développement est présente mais reste difficile à concrétiser. Est-ce-que le choix de cette politique est propice aux opportunités de développements de certaines entreprises ou certains secteurs ? la sélection des entreprises par taille est par niveaux technologiques a joué un rôle lors des travaux de Chen Wu et Lin (2006) 
Chapitre 3 Cadre conceptuel de la recherche

en Taiwan. D’autre part la collaboration université-entreprise et institut de recherche ne peut pas garantir le succès d'une entreprise en matière d'innovation technologique (Eom \& Lee, 2010). Au contraire, cela peut influer sur la sélection ou la direction des projets de recherche d'une entreprise.

A cet effet, la première dimension du processus nous permet d'établir la première hypothèse de recherche .

Hypothese 2: L'existence active d'agents externes et leur collaboration avec d'autres entreprises ou organisations dans le cadre d'activités d'innovation influencent les performances d'innovation positives des entreprises..

\subsubsection{Les instruments politiques publics de l'innovation}

Le but des décideurs politiques est de concevoir des instruments d'innovation qui produisent avec un succés des résultats sur l'innovation et de la performance au sein des entreprises (Edler \& Fagerberg, 2017). En effet la politique d'innovation fonctionne par objectif, car le design de la politique d'innovation est appliqué par le minitère ou des agences spécifiques publiques à l'échèle régionale ou nationale (Edler, Gök, Cunningham, \& Shapira, 2016, p. 3). Premiérement la politique d'innovation est implementé du point de vue de l'échec du marché (Laranja, Uyarra, \& Flanagan, 2008); cette approche prend en considération les output, input avec l'activité d'innovation. Deuxiémemement du point de vu systemique (Kuhlmann et al., 2010; Smits \& Kuhlmann, 2004) où la génération des connaissances est conçue à partir des interactions entre les acteurs (B.- $\AA$. Lundvall, 2016). Cette politique d'innovation est liée au fonctionement du système d'innovation (Bergek, Hekkert, \& Jacobsson, 2008) et le rôle des décideurs politiques est d'intervenir à travers les instruments politiques (Borrás \& Edquist, 2013). En effet, le système et ces acteurs doivent avoir la réglementation et l'appui financier approprié et bénéficier des subventions et support afin de surmenter les échecs en matière de capacité et de coopération (Edler et al., 2016, p. 5)

Edler et al., (2016, p. 11) expliquent les instruments politiques d'innovation vis-à-vis de l'offre et de la demande. Ils déterminent quinze instruments répartis en cinq objectifs politiques: Premiérement, la création de nouvelles connaissances et d'innovation du point de vue financier (P Cunningham, Gok, \& Laredo, 2012; Paul Cunningham, Gök, \& Larédo, 2016; Larédo, Köhler, \& Rammer, 2016). Deuxiémement, des supports aux capacités et compétences afin de garantir la génération et la commercialisation de l'innovation ; cela est implementé selon les besoins d'apprentissage aux sein du système d'innovation (Jones \& Grimshaw, 2016; Ramlogan \& Rigby, 2014; Shapira \& Youtie, 2016). Troisiémement, des instruments qui supportent tout type d'interaction et d'apprentissage à l'échèle nationale ou regionale (P. Cunningham \& Gök, 2016; Paul Cunningham \& Ramlogan, 2016; Diez, 2001; Uyarra \& Ramlogan, 2012, 2016). Quatrièmement, la normalisation et la régulation ont un impact sur l'offre ou la demande et sur les initiatives à prendre (Blind, 2016; Edler, 2013; Rigby, 2016; Roy Rothwell, 1980; Uyarra, 2016). En fin, la prévision technologique est une approche que les déci- 
deurs politiques utilisent afin de prévoir les tendences et les répercusions technologiques (Georghiou, 1998).

Est-ce que les instruments politiques sont influencés par l'activité d'innovation ? Comment doit-on construire ces instruments dans les pays en développement ? PadillaPerez et Gaudin (2014) montrent que les gouvernements d'Amérique centrale ont créé des organisations et des institutions publiques pour soutenir la science, la technologie et l'innovation et dont la fonction principale est d'implémenter des lois, des plans nationaux et l'élaboration d'instruments politiques. Par contre, les études des routines de gouvernance orientées vers le soutien aux activités économiques dont la majorité est appliquée à l'exploitation des ressources naturelles avec un faible intérêt pour les produits à forte valeur ajoutée ont montré une faible capacité institutionnelle. En l'occurrence, selon Su et Wu (2015) l'implémentation d'un instrument politique doit être conçue comme: un programme visant spécifiquement à promouvoir l'utilisation, l'adaptation, la production, la diffusion et l'innovation, ou en général à diriger les changements institutionnels et les développement culturels. A cet effet, la conception d'une politique locale du gouvernement est nécessaire. Ainsi la mise en œuvre, l'évaluation des instruments politiques et les outils d'implémentations sont une démarche primordiale à suivre.

Quelle sont les outils efficaces à une bonne conception d'instruments politiques ? Parmi les divers instruments de politique, deux ont le plus contribué à encourager les entreprises et à consacrer plus de ressources. Premièrement, ceux qui encouragent les entreprises à consacrer plus de ressources à la $R \& D$ et deuxièmement, l'incitation fiscale et la subvention à la $\mathrm{R} \& \mathrm{D}$ en réduisant le passif fiscal de l'entreprise (Etzkowitz \& Brisolla, 1999, p. 341). La politique consacrée à la promotion de la R\&D n'est pas adaptée aux pays en développement du fait qu'ils ont une faible capacité à assimiler la technologie et le coup de la R\&D est très élevé généralement par rapport au faible revenu de ces pays.

Comment peut-on instaurer une politique de promotion de la R\&D dans un pays faible en matière de compétences scientifiques et en ressources financières ? Salazar (2017, p. 252) évoque la problématique du choix et l'optimisation des instruments politiques en introduisant la conception de l'ouverture et le feedback, dont les éléments sont: $\mathrm{La}$ capacité de penser, de concevoir et de mettre en œuvre des politiques, une coopération internationale, un apprentissage, l'innovation et l'ouverture et la transparence et l'efficacité dans l'allocation des ressources et finalement la diffusion de l'information. Ces éléments constituent la gouvernance qui représente la clef de l'instauration d'une bonne politique.

Donc, la politique d'innovation n'est pas seulement injectée les instruments. La politique d'innovation est un état d'esprit de collaboration et d'implication. Gabriela Dutrénit et Puchet (2017) suggèrent que la politique d'innovation doit être basée sur une stratégie. La politique d'innovation est : d'instaurer les règles du jeu et les conditions 
initiales qui s'adaptent au système, prendre en considération les capacités nationales et l'état du tissu institutionnel, trouver un accord à partir des différentes conceptions, et finalement, concentrer les efforts à long terme vers le développement économique et l'inclusion sociale. Les instruments politiques sont le reflet d'une étude stratégique des moyens et des capacités. Ainsi l'implémentation des instruments suit une perception à long terme.

La politique d'innovation doit mettre l'accent sur la dimension sociétale. Srinivas et Sutz (2008b) expliquent que l'innovation technologique est mesurée par les conditions socio-économiques du pays. En effet, le manque des ressources financières dans les pays en développement, et l'état critique du niveau de vie ne permet pas aux entreprises de produire dans les mêmes conditions. Dès lors, une adaptation de la rareté des ressources doit être suivie par l'apport de plus d'attention à la nature du processus d'innovation, à l'effet de l'apprentissage, par le renforcement des capacités et les flux des connaissances entre les acteurs de l'écosystème de l'innovation, à augmenter les niveaux d'engagement avec des acteurs extérieurs au monde académique et à changer les méthodes utilisées dans la recherche. Dès lors, la politique d'innovation est construite sur la base de la génération des connaissances et la compréhension des liens qui existent entre les acteurs ; à partir de là les instruments son établis.

La politique d'innovation est menée par une stabilité des décisions et une bonne gestion des conflits sociaux. Kuhlmann et Ordóñez-Matamoros (2017, p. 15) constatent certains obstacles qui affectent le système d'innovation et diminuent le degrés de confiance entre les acteurs. En effet, Le manque de ressources économiques, techniques, juridiques et d'infrastructures et les organisations et procédures politiques peu fiables à cause de la corruption, amènent au manque de confiance dans les politiques publiques. En conséquence, une évaluation gouvernementale des connaissances, de la science, de la technologie et de l'innovation, est exigée pour le développement des entreprises, les institutions et les organisations qui participent dans l'innovation.

Les instruments politiques dans notre modèle sont inspirés de l'enquête Canadienne de 2017 «Integrated Business Statistics Program (IBSP) Survey of Innovation and Business Strategy» sur la stratégie et l'innovation dans les entreprises. Les instruments politiques dans notre modèle incluent les programmes gouvernementaux suivants :

a) Programmes d'incitation fiscale gouvernementale ou de crédit d'impôt. Ce programme inclut des programmes destinés à soutenir des activités d'innovation telles que la recherche et le développement ou les dépenses en immobilisations. L'application de cet instrument diffère selon l'activité et le type d'innovation que procure l'entreprise. A cet effet Govindaraju et al.,(2013) examinent l'importance du rôle du gouvernement sur l'innovation organisationnelle et de l'incidence des exportations sur l'innovation en matière de produits et de procédés. Ils aperçoivent que certaines incitations fiscales technologiques et non technologiques diffèrent selon l'activité de l'entreprise. En l'occurrence, Radas 
et al., (2015) indiquent l'existence de limitations dans l'utilisation des incitations fiscales par les PME. A cet effet les incitations fiscales doivent être orientées selon l'activité de l'entreprise et les besoins.

b) Les programmes de subvention pour le développement et la commercialisation de la propriété intellectuelle sont des instruments dont l'utilité s'est avérée intéressante pour les pays en développements ou pour les secteurs tardifs. A cet effet, Kim (2015) suggère que la politique gouvernementale doit encourager et faciliter le développement, la diffusion et la commercialisation des innovations de consommation. En outre, Meesapawong et al., (2014) révèlent que l'orientation commerciale réalise le score d'impact le plus élevé sur les facteurs d'innovation en Thaïlande. En conséquence, l'amélioration de la recherche et l'optimisation du potentiel de la propriété intellectuelle doivent être garanties par le gouvernement (Payumo et al., 2012). A cet effet, la promotion du développement et la commercialisation de la propriété intellectuelle doit être soutenue de la création jusqu’à la commercialisation.

c) Les programmes de formation et d'embauche doivent être conçus sur une étude de la demande et des besoins vers les connaissances. C'est pourquoi, Arocena et Sutz (2010) suggèrent que la politique est rentable lorsque l'ensemble des politiques d'innovation sont conçues comme des politiques sociales, capables de répondre à une forte demande sociale de connaissances et de développer des capacités d'innovation dans les pays en développement. En outre, Khayyat et Lee (2015) ont développé un outil de mesure qui caractérise l'innovation dans les pays en développement à partir de la promotion de l'éducation, l'enseignement, et la recherche scientifique. Ils recommandent au gouvernement d'allouer les capacités technologiques à partir des dépenses, de promouvoir des politiques de récompenses nationales pour les scientifiques et les chercheurs, de modifier les programmes scolaires, d'alléger les impôts sur les sociétés afin d'encourager les organisations locales à mener des programmes de formation spécialisés pour promouvoir les activités d'innovation. Alors, la politique d'innovation doit être implémentée dans deux sens, développement des capacités technologiques et implémentation d'instruments adaptés et orientés.

d) Les marchés publics sont gérés par le gouvernement. Bartelset al., (2012) insistent sur la mise en place par le gouvernement d'un environnement institutionnel qui soutient les marchés et les transactions commerciales, et par la suite, soutient le système national d'innovation et la croissance économique

e) L'accès aux installations est un instrument de soutien à l'innovation dans les entreprises. De là, Avilaet al., (2014) suggèrent que le programme d'appui aux technologies de communication et d'information du gouvernement procure un effet positif sur l'innovation en Andalousie en Espagne. Egalement, Jun et al., 
(2013) déduisent que le programme de soutien à la cartographie routière des PME en Corée a un effet positif sur l'innovation. A cet effet, l'implication de l'état dans le développement de l'infrastructure au service des entreprises est considérée comme une contribution au renforcement des capacités de l'entreprise.

f) Les incitatifs à l'exportation sont un facteur de croissance économique à travers l'exportation. Les chercheurs Rasiahet al., (2013) aperçoivent que l'incitation à l'exportation est accompagnée par la modernisation technologique des moyens de production. Cette relation a un effet direct sur l'augmentation des emplois.

g) L'assistance technique est représentée comme un outil d'aide au lancement d'une entreprise. A cet effet, Butleret al., (2016) ont étudié l'impact de l'assistance technique sur la promotion des sturt-ups innovantes, à Buenos Aires en Argentine. En l'occurrence, Archibugi et Pietrobelli (2003) ont étudié l'impact des différentes formes de mondialisation de la technologie sur les pays en développement. En conséquence, les pouvoirs publics peuvent aider les entrepreneurs à surmonter un grand nombre d'obstacles dans leurs entreprises et à améliorer la répartition de leurs talents entrepreneuriaux.

Cela conduit à la deuxième hypothèse.

Hypothèse 3: La politique publique développe des instruments politiques pour promouvoir l'innovation dans les institutions et les organisations et assure le bon fonctionnement du système d'innovation. Par conséquent, les instruments de la politique d'innovation contribuent positivement aux performances d'innovation des entreprises.

\subsubsection{Les résultats d'innovation}

La dernière dimension, «les résultats d'innovation» est interprétée par l'apprentissage (B.-A. Lundvall, 1985) à travers l'interaction entre les acteurs. Les acteurs apprennent à travers leur expérience au moment de collaboration ou d'interaction avec d'autres acteurs (Kuhlmann et al., 2010). En conséquence les chercheurs apprennent à travers leurs recherches et expérimentations, les entrepreneurs et les instituts apprennent de la politique instaurée et des résultats de recherche aussi les décideurs politiques apprennent à travers des collaborations et d'évaluations de la politique publique.

Les interactions entre les acteurs contribuent aux résultats et aux performances des entreprises. Choi et Williams (2013) montrent que la relation entre l'innovation et la performance de l'entreprise se caractérise par l'intensité de l'innovation, l'ampleur de l'innovation et ses répercussions sur la performance des entreprises. A cet effet, l'approche de mesure de l'innovation dans les entreprises se base sur l'élaboration d'une stratégie politique basée sur les données que diffusent ces entreprises. Dans ce cadre, A. Nelson et al., (2014) déclarent, à travers des recherches sur la chimie verte, 
l'existence d'une surestimation et une sous-déclaration des mesures de l'innovation populaire et des modèles d'innovation réels. Et par la suite ce phénomène a conduit à une sous-évaluation ou une surestimation de la diffusion.

L'adaptation de la recherche scientifique aux besoins des entreprises. Les politiques gouvernementales encourageant les capacités d'innovation nationales fondées sur la recherche. En outre, la recherche scientifique joue un rôle important pour le développement économique et le développement des systèmes d'innovation (Toivanen, 2014). L'aspect de la recherche scientifique accentue les capacités des entreprises dans le développement.

Pourquoi certaines entreprises sont plus innovantes et développent plus de projets que d'autres ? Turró et al., (2014) expliquent que ce sont les factures environnementaux qui conditionnent l'innovation au sein des entreprises. L'étude détermine l'effet modérateur des valeurs culturelles sur l'entreprenariat des entreprises. L'orientation des entreprises vers un type d'innovation suit les facteurs d'environnement et les valeurs culturelles.

L'entreprise innovante en matière d'innovation dans le produit selon le secteur ou l'activité de production. L'orientation de l'entreprises dépend du niveau de compétence en matière de compétitivité, de productivité et d'innovation (Carayannis \& Grigoroudis, 2014).

D'autre part, l'innovation dans les services est menée dans quatre dimensions: premièrement, l'intégration des services dans les systèmes nationaux d'innovation; deuxièmement, les compétences et les préférences; troisièmement, la mise en réseau et la coopération; et quatrièmement, les résultats en termes d'impact socioéconomique (Rubalcaba, Aboal, \& Garda, 2016). L'importance des spécificités dans l'innovation des services suggèrent des implications politiques et managériales.

A cet effet, l'efficacité des politiques est étudiée à travers le comportement dynamique de l'innovation du produit et de l'innovation des processus (Samara, Georgiadis, \& Bakouros, 2012). Une politique d'innovation alternative est suggérée dans les pays en développement. Alors, que l'innovation dans l'organisation et le marketing amène à l'efficacité de l'entreprise en matière de production et de commercialisation. Caniëls et Romijn (2008) évoquent l'exemple de la gestion stratégique des niches dans le cadre du développement durable. Cette gestion stratégique est conçue pour faciliter l'introduction et la diffusion de nouvelles technologies via des expériences sociétales protégées dans des domaines tels que l'énergie éolienne, le biogaz, les systèmes de transport public, le transport de véhicules électriques et la production alimentaire respectueuse de l'environnement. Cette nouvelle perception de l'innovation permet aux entreprises de se développer.

En ce qui concerne, la production en matière de brevet, licence et propriété intellectuelle, elle est une stratégie qui est mondialisée. L’internationalisation de la R\&D dans les entreprises conduit à l'émergences de nouvelles stratégies politiques (Guimon, 
2011). À travers l'analyse des brevets délivrés par l'organisation mondiale de la propriété intellectuelle (OMPI), on arrive à cartographier les systèmes d'innovation. Ainsi, l'analyse du réseau social indique quelle activité d'innovation se produit dans un pays et quels domaines technologiques sont susceptibles de donner naissance à des produits innovants dans un avenir proche (Dolfsma \& Leydesdorff, 2011). A cet effet, la politique d'innovation a pour objectif de développer des capacités industrielles dans des secteurs essentiels (Guennif \& Ramani, 2012). En outre, la production en matière de brevet, licence et propriété intellectuelle diffère entre les pays développés et les pays en voie de développement et par la suite les conditions de la protection de la propriété intellectuelle aussi (Spielman \& Ma, 2016). Donc on peut dire que le choix de l'innovation est affecté par l'environnement et l'intérêt politique ; les effets varient d'une culture à l'autre.

Selon le Canadien de 2017 «Integrated Business Statistics Program (IBSP) Survey of Innovation and Business Strategy» et du questionnaire «The Community Innovation Survey» (CIS) du 2014, l'innovation est considérée comme: innovation en matière de biens (produits), innovation de service, innovation dans le processus, innovation organisationnelle, innovation marketing et finalement, brevet, licence, propriété intellectuelle selon le CIS 2014.

\subsubsection{Obstacles et freins à l'innovation}

Enfin, il convient de noter brièvement que les entreprises perçoivent certaines barrières et obstacles à l'innovation. On peut les comprendre comme une perception "ex ante" ou aussi comme une expérience "ex post". La première est vécue par les entreprises qui n'innovent pas et la seconde par les entreprises qui ont cette expérience (D Este et al., 2012). Selon l'enquête de l'ECI (Commission européenne, 2014), celles-ci, entre autres, sont notées. Le premier groupe comprend les éléments suivants : Manque de financement interne, manque de crédit public ou privé, incertitude et risque, et politique d'innovation inadéquate. Dans le second groupe : manque de partenaires de collaboration, manque de personnel qualifié, demande et taille du marché, concurrence excessive sur le marché, compétences des entreprises et impossibilité de trouver des collaborateurs externes appropriés.

Cela nous amènerait à formuler le dernier scénario

Hypothèse 4: Les obstacles à l'innovation influenceront les performances de l'entreprise en matière d'innovation.

\subsection{Conclusion.}

En cet effet, l'apprentissage des acteurs à travers l'interaction permet au pouvoir public de bénéficier des connaissances acquises des acteurs pour réajuster la politique ou développer de nouveaux instruments qui sont mieux adaptés et favorables au fonctionnement du système d'innovation. 
Evaluation de la politique d'innovation sur un échantillon d'entreprises algériennes 


\section{Chapitre 4 Méthodologie de Recherche}


Evaluation de la politique d'innovation sur un échantillon d'entreprises algériennes 
L'objectif de ce chapitre est de décrire la méthodologie adoptée pour tester le cadre conceptuel choisi pour notre recherche. Nous abordons, dans quatre paragraphes principaux, respectivement le design de la recherche, la démarche de la collecte des données, la difficulté du terrain, la source, l'élaboration et le choix du questionnaire et les techniques statistiques que nous avons employées pour vérifier nos propositions et hypothèses de recherche.

\subsection{Le design de la Recherche}

Michael Crotty (1998) distingue quatre approches pour établir le design de la recherche: l'épistémologie, la théorie, la méthodologie et la technique choisie.

En outre, Creswell (Creswell, 2009) distingue trois types de recherche: recherche quantitative, recherche qualitative et méthode mixte ( tableau ci dessous). En effet, le choix de la méthodologie est influencé par les problèmes de la recherche, l'expérience personnelle du chercheur et le public pour lequel le rapport sera rédigé (Creswell, 2009).

Tableau 4.1 Les critères de validation du modèle PLS

\begin{tabular}{|c|c|c|c|}
\hline Ont tendance à ou généralement & Approaches qualitative & Approaches quantitative & Approaches mixtes \\
\hline $\begin{array}{l}\text { Utiliser ces hypothèses } \\
\text { philosophiques } \\
\text { employer ces stratégies d'enquête }\end{array}$ & $\begin{array}{l}\text { Revendications des connaissances } \\
\text { constructivistes/de plaidover / } \\
\text { participatives } \\
\text { phenoménologie, theorie enracinée, } \\
\text { ethnographie, étude de cas et } \\
\text { narration }\end{array}$ & $\begin{array}{l}\text { Connaissances post positivistes } \\
\text { enquêtes et expériences }\end{array}$ & $\begin{array}{l}\text { Revendications de connaissances } \\
\text { pragmatiques } \\
\text { séquentiel simultané et } \\
\text { transformatif }\end{array}$ \\
\hline Employer ces méthodes & $\begin{array}{l}\text { Questions ouvertes } \\
\text { approches emergentes texte ou } \\
\text { image }\end{array}$ & $\begin{array}{l}\text { Questions fermées approches } \\
\text { prédéterminées données } \\
\text { numériques }\end{array}$ & $\begin{array}{l}\text { Questions ouvertes et fermées, } \\
\text { approches nouvelles et } \\
\text { prédéterminées, données et } \\
\text { analyses quantitatives et } \\
\text { qualitatives. }\end{array}$ \\
\hline $\begin{array}{l}\text { Utiliser ces pratiques de recherche } \\
\text { en tant que chercheur }\end{array}$ & $\begin{array}{l}\text { Se positionne } \\
\text { Recueille les significations des } \\
\text { participants Se concentre sur un seul } \\
\text { concept ou phénomenene } \\
\text { Apporte des valeurs personnelles à } \\
\text { l'étude } \\
\text { Étudie le contexte ou le cadre des } \\
\text { participants } \\
\text { Valide lexactitude de la constatation } \\
\text { Fait des interprétations des données } \\
\text { Crée un agenda pour le changernent } \\
\text { ou la réforme } \\
\text { Collabore avec les participants }\end{array}$ & $\begin{array}{l}\text { teste ou vérifie des théries ou des } \\
\text { explications } \\
\text { identifier les variables à étudier } \\
\text { relie des variables dans des } \\
\text { questions ou des hypothéses } \\
\text { utilise des normes de validitité et de } \\
\text { fiabilité } \\
\text { observe et mesure numériquement } \\
\text { les informations } \\
\text { utilise des approches impartiales } \\
\text { emploie des procédures statistiques }\end{array}$ & $\begin{array}{l}\text { Recueille des données quantitatives } \\
\text { et qualitatives } \\
\text { Développe une logique de mélange } \\
\text { Intègre les données à différentes } \\
\text { étapes de renquête } \\
\text { Présente des images visuelles des } \\
\text { procédures dans l'étude } \\
\text { Utilise les pratiques de la recherche } \\
\text { qualitative et quantitative }\end{array}$ \\
\hline
\end{tabular}

En l'occurrence, Saunders et al (2008, p. 108) expliquent la différence entre les différentes méthodologies en profondeurs ( figure ci dessous). 


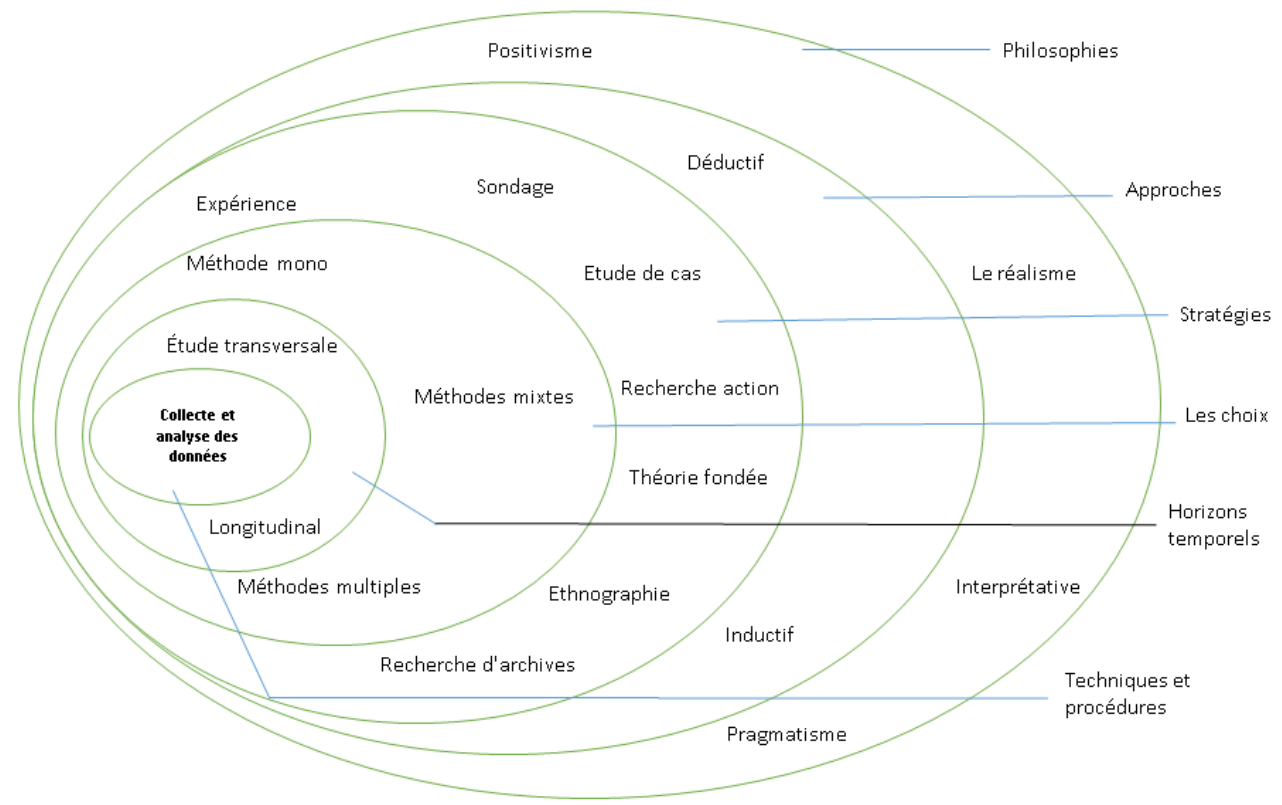

Source: Saunders et al (2008, p. 108)

Figure 13 Le processus de la recherche oignon

En conséquence, on a opté pour la méthodologie quantitative déductive, car cette méthode s'adapte premièrement à la problématique de recherche, puisqu'on sait clairement à l'avance les éléments du système ; on veut seulement confirmer et généraliser nos connaissances, car cette méthode nous permet de confirmer les hypothèses de recherche choisie dans notre étude et d'une manière scientifique dont les résultats sont pertinents et significatifs (Abdelhammid \& Samir Baha Eddine, 2019; Srholec, 2011). Deuxièmement, elle est choisie par motivation personnelle, car ce n'est pas premièrement la fois que le sujet sur l'innovation est entamé. À cet effet, dans notre démarche nous essayons de regrouper tous les éléments de l'innovation pour avoir une image globale du système d'innovation. Finalement, il y a très peu de revues dans la littérature portant sur le système d'innovation en Algérie et il y a visiblement un manque de données; c'est pour cela que nous avions choisi de collecter des données et utiliser des moyens statistiques d'analyse. 


\subsection{La démarche de la collecte des données}

Nous avons suivi une démarche d'analyse dans tout le processus de notre recherche. Les étapes que nous avons suivies nous ont motivées pour opter pour la collecte des données à travers des questionnaires (Brandt, Thun, Thunb, \& Thun, 2016; OCDE, 2016; Ramlogan \& Rigby, 2014). La démarche est la suivante:

a) Prise de rendez-vous avec le responsable de l'innovation et de la compétitivité au ministère de l'industrie et des mines en Algérie. Et cela dans le cadre du projet de jumelage sur l'innovation entre l'Algérie, la France l'Espagne et la Finlande, pour le transfert des connaissances et d'expériences des pays du nord vers les pays du sud. Ces entretiens nous a permis de connaitre les préoccupations des décideurs politiques en vu d'une implantation d'une stratégie et d'une politique optimale pour l'industrie en Algérie. D'où la motivation de la problématique de recherche.

b) Prise de contact avec le ministre de l'industrie et des mines au niveau spécialement de la région Mostaganem. L'entretien, nous a permis d'avoir une idée sur les programmes de subvention à l'innovation et sur le retour des entreprises. La collaboration entre les entreprises et le ministère est presque inexistente. De là, nous avons établi les hypothèses de nôtre recherche.

c) Prise de contact avec le directeur de la chambre de commerce de Mostaganem, qui nous a fait savoir que la connexion avec les entreprises est faible. Car ces dernières ne sont pas motivées à collaborer, surtout en ce qui concerne la transparence par exemple.

d) Entretien avec les responsables au niveau du ministère de l'industrie et des mines. Nous avons constaté que les données sur les entreprises et surtout en ce qui concerne l'innovation sont très compliquées à avoir. Les statistiques du ministère sont basées sur des données approximatives provenant d'autres entités. Cette étape nous a motivé à choisir de collecter les données à travers le sondage.

e) Nous avons choisi le secteur pharmaceutique pour son importance au niveau politique et technologique en Algérie et nous avons adressé un questionnaire aux entreprises de celui-ci.

f) Après, nous avons essayé de contacter les experts qui ont répondu au questionnaire afin d'analyser les détails des resultats de l'etude quantitative.

\subsection{Difficulté du terrain}

Nous avons construit le questionnaire étape par étape en analysant le terrain comme suit : 
Premièrement, nous avons entamé des interviews avec les responsables des entreprises pharmaceutiques : représentants médicaux, commerciaux, responsables de production, responsables export /import et finalement les managers de la région d'Oran. Les questions ont été basées sur la revue de littérature sur l'innovation et inspirées à partir du questionnaire Canadien de 2017 «Integrated Business Statistics Program (IBSP) Survey of Innovation and Business Strategy» et du questionnaire «The Community Innovation Survey» (CIS) du 2014. Cette démarche nous a permis de comprendre la vision des entreprises vis-à-vis de l'innovation.

Deuxièmement, le questionnaire est établi online et adapté selon la vision des entreprises.

Troisièmement, nous avons essayé de contacter les entreprises pharmaceutiques à travers la base de données de la chambre de commerce Algérienne. Il y avait un manque d'informations concernant les numéros de téléphone et les emails de contact.

Quatrièmes, Nous avons complété notre recherche à travers « les pages maghreb » et ensuite adressé le questionnaire à toutes les entreprises pharmaceutiques algériennes se trouvant sur leur liste. Au final nous avons récolté seulement deux retours de la part de deux entreprises import export qui n'étaient pas de toute évidence notre cible principale.

Enfin, nous avons contacté les entreprises à travers Linkedin; et nous avons eu des retours.et ensuite ils ont accepté d'avoir des entretiens personnelles.

\subsection{Sources de données}

Les données utilisées pour l'analyse empirique ont été recueillies en Algérie. Le travail visait à comprendre l'impact des décisions politiques d'innovation sur les résultats de l'innovation dans les entreprises. L'adaptabilité des instruments politiques aux exigences des entreprises à travers les acteurs d'innovation et les activités d'innovation implique un succès de la politique publique d'innovation. L'analyse a été menée auprès de 67 entreprises pharmaceutiques.

Selon les statistiques de la chambre de commerce algérienne (2018), le secteur pharmaceutique Algérien est constitué de 140 entreprises dont 71 sont des entreprises pharmaceutiques de production. Plus de $57 \%$ de ces entreprises sont des petites et moyennes entreprises (CHOUAL, 2016).

L'élaboration des questions a été basée sur la revue de littérature sur l'innovation et inspirées à partir du questionnaire Canadien de 2017 «Integrated Business Statistics Program (IBSP) Survey of Innovation and Business Strategy» et du questionnaire «The Community Innovation Survey» (CIS) du 2014. La collecte des données quantitatives a été réalisée online sur la page web (Dillman, 2007). Le Survey nous permet d'étudier les facteurs principaux conduisant à la prise de décision politique sur l'innovation. Les données quantitatives permettent la généralisation via l'application d'un test permettant 
de vérifier les hypothèses de façon rigoureuse (Kotrlik \& Higgins, 2001). Nous avons reçu 114 réponses mais seulement 67 d'entre elles sont valides. L'étude consistait à l'analyse des réponses des 67 entreprises qui ont répondu au questionnaire. On s'est basé sur ce nombre-là parce que certains répondants non pas remplis certaines cases donc il y a que 67 responsables qui ont pué répondre à toutes les questions poser. L'enquête a été faite entre les mois de juin et novembre 2018. La majorité des répondants est localisée dans la capitale.

En plus de ça, nous avons pue faire 25 interviews avec les répndant. Cela dans le but de confirmé nos résultats auprès les analyses quatitatives à travers des réponses

\subsection{Elaboration et choix du questionnaire}

Le questionnaire comprend six composantes principales avec des possibilités de réponses dichotomiques avec plusieurs choix (Annexe 1):

a) L'information générale sur l'entreprise (l'adresse, la position des répondants, l'âge de l'entreprise, effectifs de l'entreprise)

b) Les acteurs qui correspondent aux relations qui existent entre l'entreprise et l'écosystème (collaboration entre entreprises du même groupe, fournisseurs d'équipements, de matériaux, de composants ou de logiciels, des clients ou fournisseurs du secteur public, des clients ou fournisseurs du secteur privé, concurrents ou autres entreprises de votre secteur, consultants ou laboratoires commerciaux, universités, gouvernement, instituts de recherche publics ou privés).

c) L'instrument politique incluant les subventions de l'état dans le cadre de la promotion de l'innovation au sein des entreprises pharmaceutiques en Algérie (Les programmes d'encouragements fiscaux, Les programmes de subvention pour le développement et la commercialisation de la propriété intellectuelle, Les programmes de formation et d'embauche, Les marchés publics, L'accès aux installations, Les incitatifs à l'exportation, L'assistance technique).

d) L'activité d'innovation que l'entreprise entreprend pour innover ou améliorer le produit : R\&D interne, R\&D externe, Acquisition de machines, d'équipements, de logiciels et de bâtiments, Acquisition des connaissances détenues par d'autres entreprises ou organisations, Formation pour des activités innovantes, Introduction sur le marché des innovations (les études de marché et la publicité de lancement), Activités internes ou sous-traitées pour modifier la forme, l'apparence ou la facilité d'utilisation de biens ou de services, Licence ou achat d'un brevet, Utilisation avancée de la technologie.

e) Les résultats de l'innovation (Innovations en matière de biens) : produits, Innovation de service, Innovation dans le processus, Innovation organisationnelle, Innovation marketing, Brevet, licence, propriété intellectuelle. 
f) Les barrières à l'innovations: Manque de financement interne pour l'innovation, Manque de crédit ou de capital-investissement, Manque d'employés qualifiés dans l'entreprise, Difficulté à obtenir des subventions gouvernementales ou des subventions pour l'innovation, Manque de partenaires de collaboration, Demande du marché incertaine pour vos idées d'innovations, Trop de concurrence dans le marché, Taille du marché, Manque de compétences, Trouver et conclure des accords avec des collaborateurs externes, Incertitude et risque, Politique de concurrence réglementaire ou gouvernementale, Protection de la propriété intellectuelle. En ce qui concerne les propositions à la politique adaptée à l'innovation au sein de l'entreprise, les facteurs sont inspirés des instruments politiques (Manque de financement interne pour l'innovation, Manque de crédit ou de capital-investissement, Manque d'employés qualifiés dans votre entreprise, Difficulté à obtenir des subventions gouvernementales ou des subventions pour l'innovation, Manque de partenaires de collaboration, Demande du marché incertaine pour vos idées d'innovations, Trop de concurrence dans votre marché, Taille du marché, Manque de compétences, Trouver et conclure des accords avec des collaborateurs externes, Incertitude et risque, Politique de concurrence réglementaire ou gouvernementale, Protection de la propriété intellectuelle).

g) Les barrières à l'adoption de la technologie : Manque de conviction, difficulté à obtenir un financement, coût, investissement non nécessaire, manque de compétences techniques, absence de culture organisationnelle, décisions prises par des sociétés mères, manque de support technique, manque d'information, difficulté à l'intégration.

\subsection{Les techniques Statistiques pour l'Analyse des Données}

\subsubsection{La Modélisation par Équation Structurelle :’' L’Approche PLS’' (Partial Least Squares)}

À cette fin, nous avons appliqué l'approche PLS qui est une estimation appropriée, étant donné son avantage sur la méthode quantitative, de permettre un test statistique formel de nos hypothèses théoriques.

L'approche Partial Least Square (PLS) nous permet de construire des variables latentes proxy qui sont mesurées par un ou plusieurs indicateurs. Premièrement, on peut utiliser le modèle dans différentes situations et sans avoir des problèmes de tailles d'échantillon. Deuxièmement, il peut comporter plusieurs variables car il peut estimer des modèles complexes. Finalement, le modèle se base sur des hypothèses moins strictes concernant la distribution des variables et les termes d'erreur. (Jörg Henseler, Ringle, \& Sinkovics, 2009, p. 283)

La PLS a été développée principalement par Wold (H Wold, 1974, 1985; Herman Wold, 1982). C'est une famille d'algorithmes ou de prescriptions par moindres carrés 
alternés. Le modèle se base sur l'analyse en composantes principales et l'analyse de la corrélation canonique.

Les modèles de chemin PLS sont définis formellement par deux équations linéaires soft: le modèle interne et le modèle externe. Le modèle interne spécifie les relations entre les variables non observées ou latentes, tandis que le modèle externe spécifie les relations entre une variable latente et les variables observées ou manifestes. (Jörg Henseler et al., 2009, p. 285).

La taille de l'échantillon dans le modèle PLS est soit égale au plus grande à dix fois le nombre d'indicateurs de l'échelle avec le plus grand nombre d'indicateurs formatifs, ou à dix fois le plus grand nombre de trajets de structure de données directes dans le cas du modèle de chemin d'accès interne (Lohmöller, 2013).

\subsubsection{Les critères de validation du modèle}

Il existe des critères pour la fiabilité et la viabilité du modèle. Premièrement, la fiabilité de la cohérence interne. Ce critère de la cohérence interne est aussi celui de Cronbach (Cronbach, 1951). Ce coefficient fournit une estimation de la fiabilité basée sur les intercorrélations des indicateurs. Cronbach $\alpha$ a tendance à sous-estimer sévèrement la fiabilité de la cohérence interne des variables latentes.

En l'occurrence, la fiabilité composite $p_{c}$ prend en compte le fait que les indicateurs ont des chargements différents et peut être interprétée de la même manière que celle de Cronbach (Werts, Linn, \& Jöreskog, 1974). Une valeur de fiabilité de cohérence interne, supérieure à 0,7 en début de recherche et supérieure à 0,8 ou 0,9 à un stade de recherche plus avancé, est considérée comme satisfaisante (J. C. Nunnally \& Bernstein, 1994); alors qu'une valeur inférieure 0,6 indique un manque de fiabilité.

La variable latente devrait expliquer une partie substantielle de la variance de chaque indicateur (généralement au moins 50\%). Donc, les corrélations absolues entre une construction et chacune de ses variables manifestes doivent être supérieures à 0,7. En l'occurrence, la psychométrie suggère d'éliminer les indicateurs de réflexion si la valeur est inferieure à 0.4 (Churchill Jr, 1979). 
Evaluation de la politique d'innovation sur un échantillon d'entreprises algériennes

Tableau 4.2 Les critères de validation du modèle PLS

\begin{tabular}{|c|c|}
\hline Critère & Caractéristique \\
\hline Fiabilité composé $\mathbf{p}_{\mathrm{e}}$ & $\begin{array}{l}\rho_{c}=\left(\Sigma \lambda_{i}\right)^{2} /\left[\left(\Sigma \lambda_{i}\right)^{2}+\Sigma \operatorname{Var}\left(\varepsilon_{i}\right)\right] \text { Quand } \lambda_{\text {i est outer (composant) le chargement vers un indicateur, et (B) }} \text { en cas d'indicateurs standardisés. La fiabilité composite est une mesure de la consistance interne et ne } \\
\text { doit pas être inférieure à } 0,6 \\
\operatorname{Var}\left(\varepsilon_{i}\right)=1-\lambda_{i}^{2}\end{array}$ \\
\hline Fiabilité de l'indicateur & Les charges extérieures (composants) normalisées absolues doivent être supérieures à 0,7 . \\
\hline \multirow{3}{*}{$\begin{array}{l}\text { Variance moyenne extraite } \\
\text { (AVE) }\end{array}$} & $\mathrm{AVE}=\left(\sum \lambda_{i}^{2}\right) /\left[\sum \lambda_{i}^{2}+\sum \operatorname{Var}\left(\varepsilon_{i}\right)\right]$ \\
\hline & Quand $\lambda \mathrm{i}$ est le composant le chargement vers un indit $\operatorname{Var}\left(\varepsilon_{i}\right)=1-\lambda_{i}^{2}$ \\
\hline & Dans le cas d'indicateurs standardisés. La variabilité moyenne extraite devrait être supérieure à 0,5 . \\
\hline Critère Fornell - Larcker & $\begin{array}{l}\text { Afin d'assurer la validité discriminante, l'AVE de chaque latente variable doit être plus élevée que les } \\
\text { corrélations au carré avec tous autres variables latentes. Ainsi, chaque variable latente est plus variante } \\
\text { avec son propre bloc d'indicateurs qu'avec un autre variable latent dont il représente un bloc } \\
\text { d'indicateurs différent. }\end{array}$ \\
\hline Charges croisées & $\begin{array}{l}\text { Les chargements croisés offrent un autre contrôle de validité discriminant. Si un indicateur a une } \\
\text { corrélation plus forte avec une autre variable latente qu'avec sa variable latente respective, il convient de } \\
\text { réexaminer la pertinence du modèle. }\end{array}$ \\
\hline
\end{tabular}

\subsubsection{Evaluation du modèle structurel}

Selon Chin (1998, p. 323) les valeurs $\mathrm{R}^{2}$ de $0.67,0.33$ ou 0.19 pour la variable latente endogène du modèle de chemin interne sont décrites comme substantielle, modérée ou faible.

Estimations des coefficients de chemin, Les valeurs estimées pour les relations de chemin dans le modèle structurel doivent être évaluées en termes de signe, de magnitude et de signification (cette dernière via bootstrapping).(Jörg Henseler et al., 2009, p. 303)

L'effet taille $f^{2} ; f^{2}=\left(R^{2}{ }_{\text {inclut }}-R^{2}{ }_{\text {exclut }}\right) /\left(1-R^{2}\right.$ inclut $)$; la valeur de 0.02 , 2.15 et 0.35 peut être considérée comme un indicateur permettant de déterminer si une variable prédite ou latente a un effet faible, moyen ou important au niveau structurel. (Jörg Henseler et al., 2009, p. 303).

La pertinence de la prévision $\left(Q^{2}\right.$ et $\left.q^{2}\right)$ est calculée sur la base de la procédure à l'aveugle:

$\mathrm{Q}^{2}=1-\left(\sum_{\mathrm{D}} \mathrm{SSE}_{\mathrm{D}}\right) /\left(\sum_{\mathrm{D}} \mathrm{SSO}_{\mathrm{D}}\right)$. D est la distance d'omission, SSE est la somme des carrés des erreurs de prédiction, SSO est la somme des carrés des observations. Les valeurs $\mathrm{Q}^{2}$ supérieures à zéro indiquent que les valeurs observées sont bien reconstruites et que le modèle a une pertinence prédictive (les valeurs Q2 inférieures à zéro indiquent un manque de pertinence prédictive). En correspondance avec $f^{2}$, l'impact 
relatif du modèle structurel sur les mesures observées pour les variables dépendantes latentes peut être évalué (Jörg Henseler et al., 2009, p. 303):

$\mathrm{q}^{2}=\left(\mathrm{Q}_{\text {inclut }}^{2}-\mathrm{Q}_{\text {exclut }}^{2}\right) /\left(1-\mathrm{Q}_{\text {inclut }}^{2}\right)$

La mesure de la pertinence prédictive est du Stone-Geisser (Geisser, 1974; Stone, 1974). Elle peut être mesurée à l'aide de la méthode du bandage aveugle (Tenenhaus, Vinzi, Chatelin, \& Lauro, 2005). Le critère Stone - Geisser suggére que le modèle doit pouvoir fournir une prévision des indicateurs de la variable latente endogène. La technique représente une synthèse d'ajustement de fonction et de validation croisée. Si cette valeur pour une certaine variable latente endogène est supérieure à zéro, ses variables explicatives fournissent une pertinence prédictive. Par analogie avec l'évaluation taille-effet $f^{2}$, l'impact relatif de la pertinence prédictive peut être évalué à l'aide de la mesure $q^{2}$ : valeurs de $0.02,0.15$ et 0.35 pertinence prédictive, moyenne ou grande d'une certaine variable latente, expliquant ainsi la variable latente endogène en cours d'évaluation (Jörg Henseler et al., 2009, p. 305).

\subsubsection{Le software du PLS}

PLS-Graph nous permet d'effectuer la modélisation de chemin de variable latente à l'aide de l'approche PLS (Partial Least Squares). PLS impose beaucoup moins de restrictions sur des questions telles que la taille de l'échantillon et la distribution des données (Lohmöller, 1989). PLS-Graph a été développé en tant que logiciel autonome basé sur une interface graphique permettant de créer des platines d'entrées compatibles avec le programme PLSX existant développé par Lohmoller (1989). PLS-Graph est une interface utilisateur graphique basée sur Windows qui aide à effectuer des analyses PLS. Le programme aide à générer des fichiers de commandes compatibles avec PLSX (version 1.8). PLS-Graph peut ensuite être utilisé pour traiter le fichier de commande afin de créer un fichier de sortie contenant les résultats de l'analyse spécifiée par le fichier de commande. PLS-Graph permet d'afficher le fichier de résultats dans une fenêtre déroulante ou bien d'extraire une partie des résultats dans le même graphique que celui utilisé pour générer le fichier de commande PLSX. L’utilisateur peut représenter un modèle théorique sous forme de représentation graphique conforme à la méthode de modélisation des équations structurelles par les moindres carrés partiels avec une variable latente (Chin, 2001, p. 1). 
Evaluation de la politique d'innovation sur un échantillon d'entreprises algériennes 


\section{Chapitre 5 Étude empirique sur le terrain}


Evaluation de la politique d'innovation sur un échantillon d'entreprises algériennes 


\subsection{Analyse descriptive}

\subsubsection{Information générale}

L'échantillon se compose en majeur partie des entreprises de production dont leur nombre représente $67 \%$ de l'effectif total. La cause de cette majorité est due au nombre important d'entreprises multinationales et privées qui se sont installées suite aux incitations des politiques à la production locale. En l'occurrence, l'importation et la distribution représente $16 \%$ de notre échantillon puisque la loi sur la réduction des importations en faveur de la production locale a d'une part, démotivé les importateurs et d'autre part, incité les multinationales à investir dans le territoire Algérien. Les consultants et les entreprises prestataires représentent les organisations qui participent au développement et à l'amélioration du processus de production (Figure Répartition des répondants par activité). Aussi, la majeure partie de l'échantillon est installée dans la région centre de Algérie (Figure 5.2) à cause de la centralisation des décisions politiques; les entreprises multinationales préfèrent donc investir dans la capitale ou à proximité pour les facilités administratives.

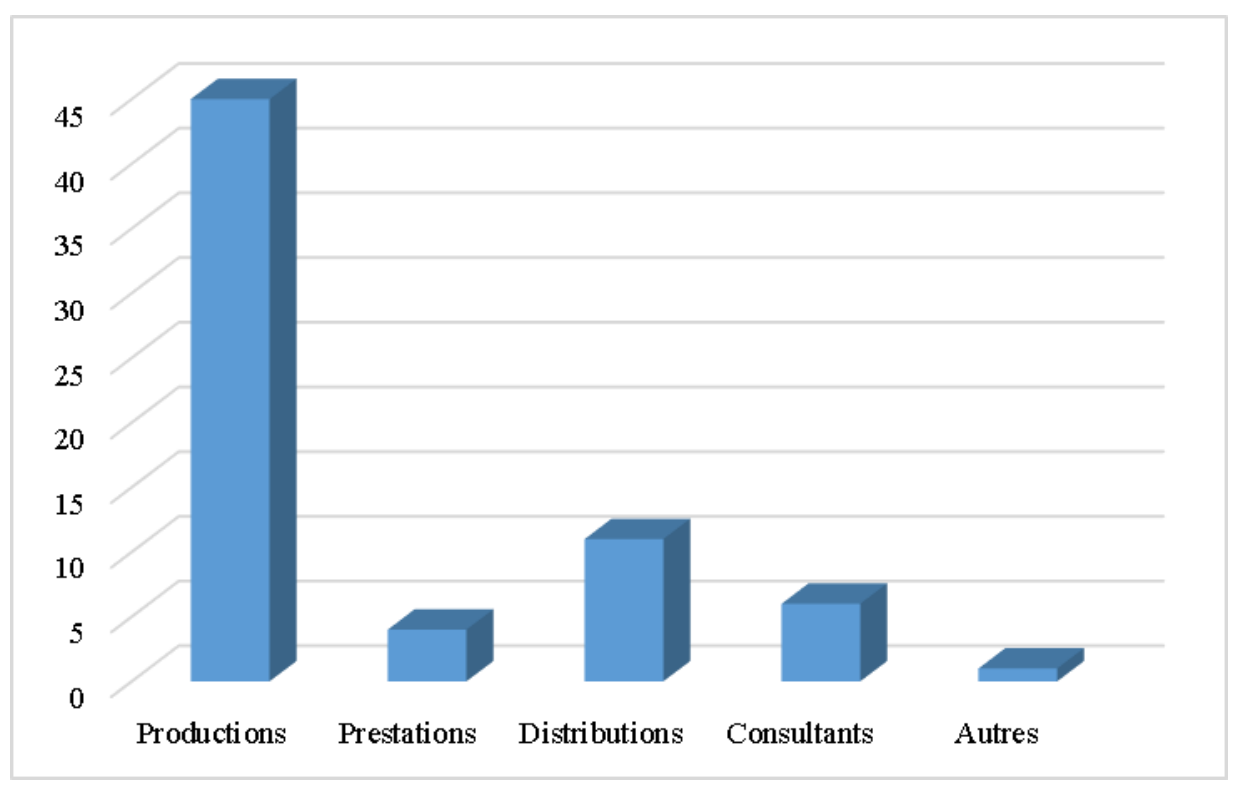

Figure 5.1 Répartition des répondants par activité 


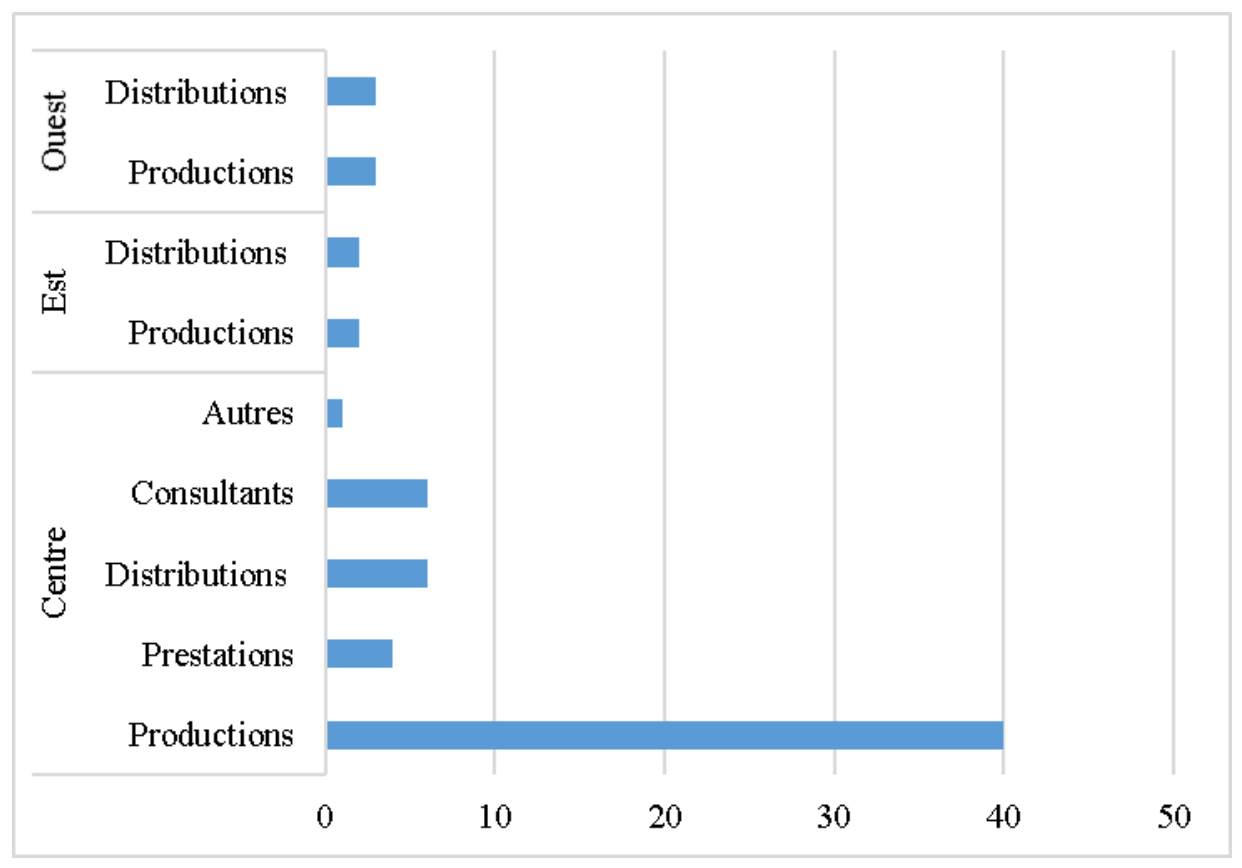

Figure 14 Répartition des répondants par région

L'échantillon analysé, comporte l'avis de l'ensemble des responsables (Figure 5.3) et décideurs du secteur (directeurs généraux et employés des différentes directions) dont la volonté de création et d'innovation est présente et concrète. En ce qui concerne l'âge de l'entreprise nous avons constaté que 31\% des répondants n'avaient pas une idée de l'âge de l'entreprise. En l'occurrence 40\% des entreprises de notre échantillon sont des entreprises pharmaceutiques qui ont plus de dix ans dans le domaine (Figure 5.4). Les entreprises du secteur pharmaceutique ayant pris naissance avec l'entreprise Saidal ont évolué à partir des expériences des anciens employés ainsi que du transfert technologique obtenu lors des collaborations avec les multinationales dans le développement des médicaments. A cet effet, le secteur pharmaceutique bénéficie de l'expérience des employés de Saidal pour consulter ou pour lancer des projets. En ce qui concerne les effectifs, les 20 entreprises pharmaceutiques nationales hormis Saidal ou Biopharm qui sont parmi les grandes entreprises en Algérie, sont en phases de développement et d'extensions. Les multinationales, par contre, sont caractérisées par un nombre important d'effectifs. La figure 5.5 montre que pour plus de $50 \%$ des entreprises, l'effectif ne dépasse pas 100 personnes. 


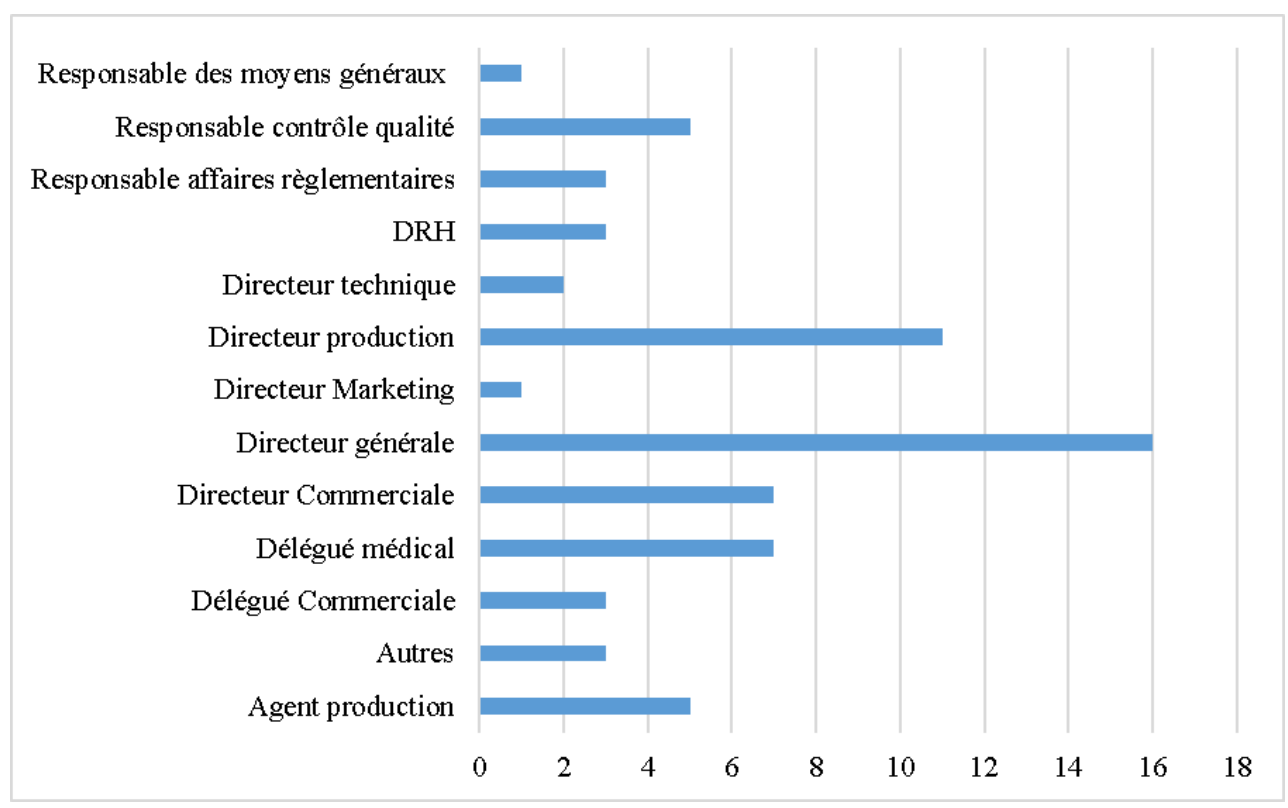

Figure 5.3 La position des répondants

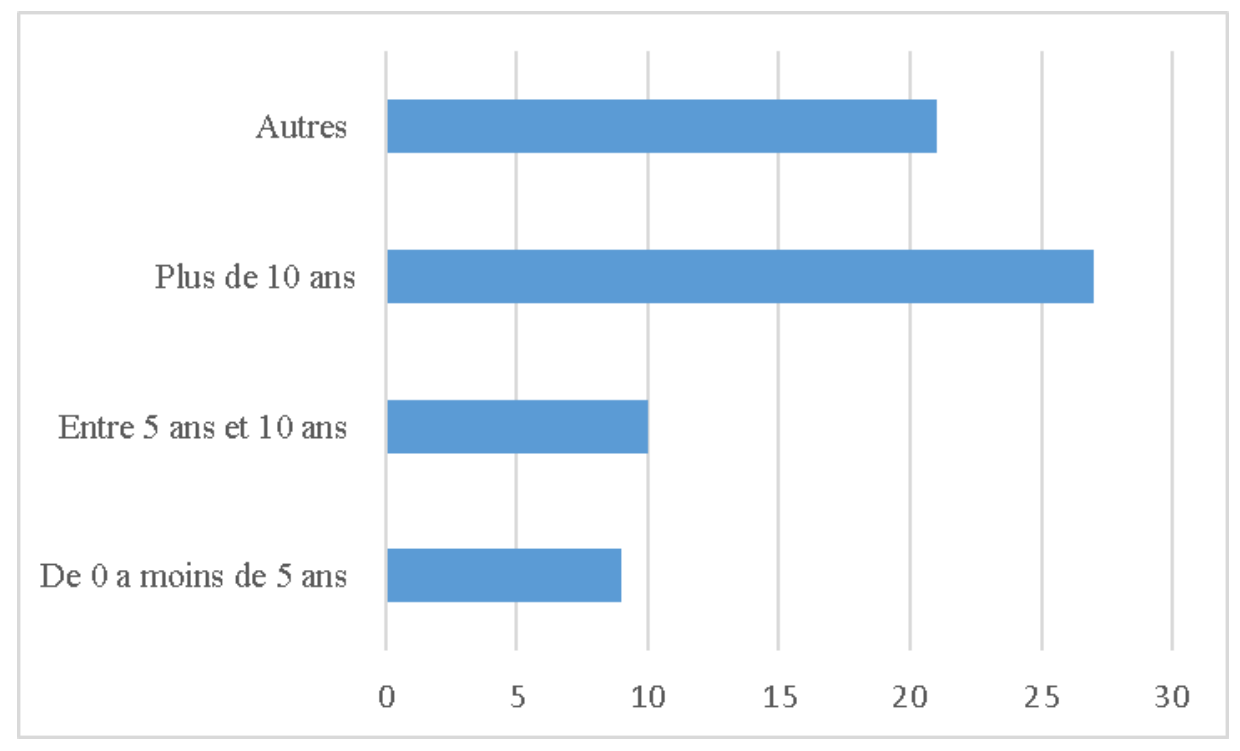

Figure 5.4 L'Age de l'entreprise 


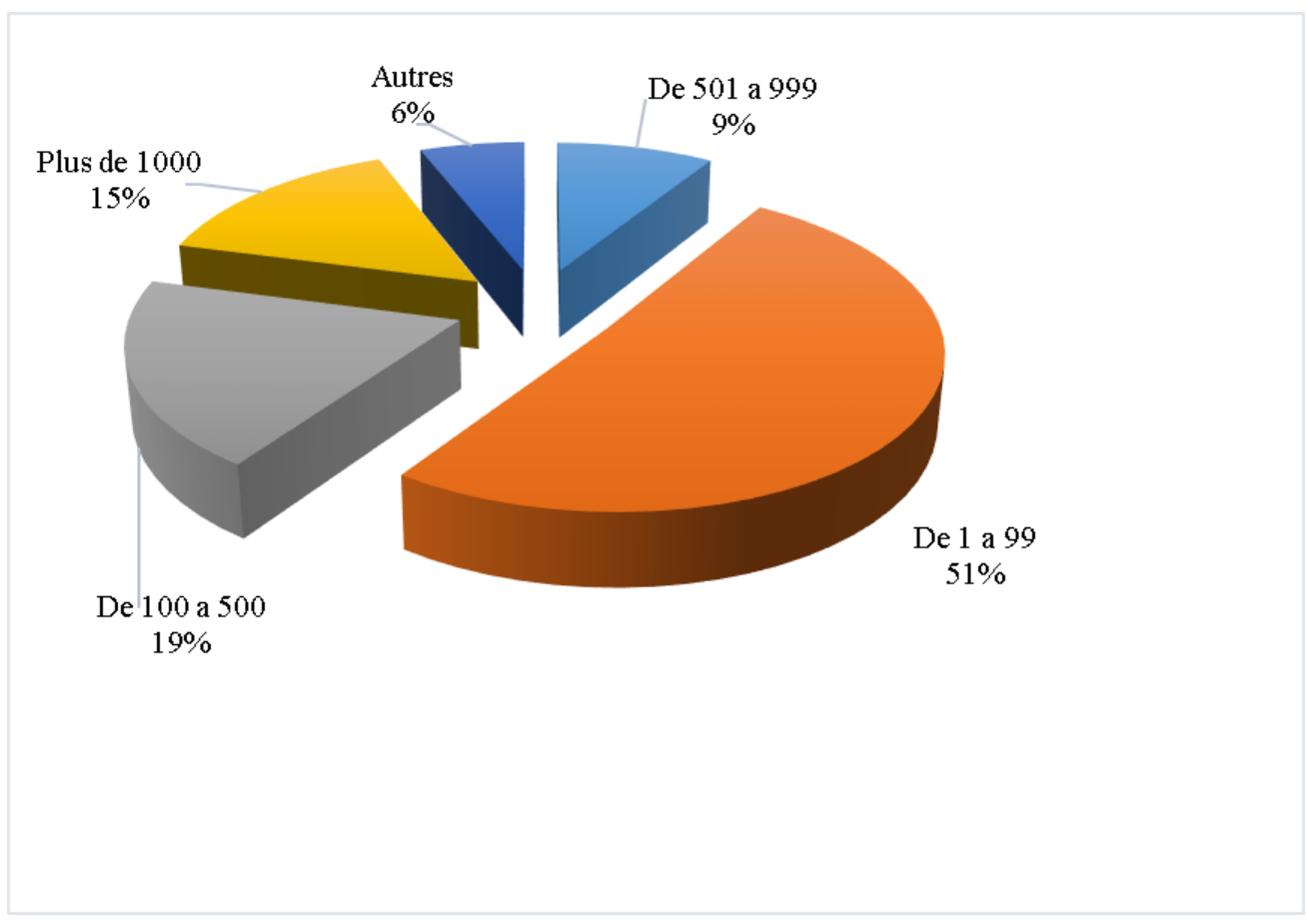

Figure 15 Effectifs de l'entreprise

\subsubsection{Les résultats (outputs) de l'innovation}

Vu l'ouverture du marché aux investissements étrangers et la promotion de la production locale par les gouvernements successifs et le développement du secteur pharmaceutique, les différents types d'innovation sont présents de façon équitable. L'innovation radicale est inexistante car la plupart des entreprises opte pour les médicaments génériques pour lesquels un grand nombre de brevets a expiré et leur appropriation est désormais accessible. Dans l'échantillon analysé, les répondants réfèrent aux Brevets, licences et propriétés intellectuelles, le développement de médicaments aux seins de leurs laboratoires avec la finalité d'atteindre et pouvoir imiter la molécule mère avec les moyens internes. En ce qui concerne l'innovation en matière de biens, les répondants optent pour le lancement de produits nouveaux qui exigent un matériel spécifique et des infrastructures adaptées. L'innovation dans le processus selon les répondants est liée aux ajustements que le directeur technique doit effectuer au sein du laboratoire pour adapter les machines et le matériel à la production quel que soit le cout de la fabrication. Dans le domaine du service organisation et du marketing, les entreprises sont très avancées en matière d'innovation car la concurrence rude dans 
l'élaboration de certains produits oblige les entreprises à être plus créatives et attentives au marché (Figure ci desous)

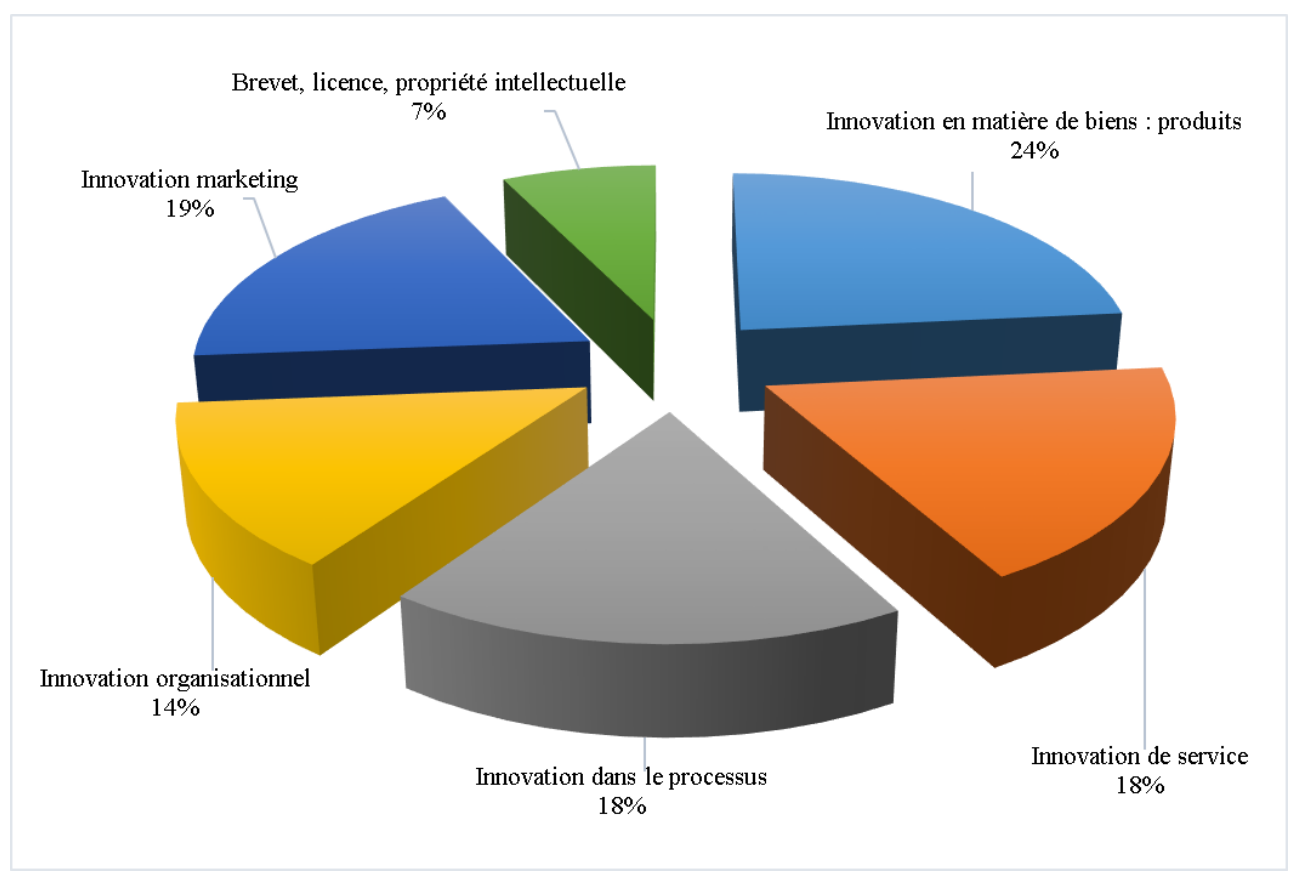

Figure 16 Les outputs de l'innovation

\subsubsection{L'activité de l'innovation des entreprises}

Les entreprises pharmaceutiques sont caractérisées par le développement interne de leur projet. 73\% des entreprises de notre échantillon optent pour la R\&D (Recherche et Développement) interne ; ni les universités ni les instituts privés ou publiques ne sont sollicités dans leur recherche et développement. Cela est dû à leur manque de connexion avec l'environnement scientifique externe. $39 \%$ des entreprises optent pour l'acquisition des connaissances externes ; souvent elles ont tendance à consulter des experts indiens qui ont prouvé leur savoir-faire dans le générique. En l'occurrence 57 $\%$ des entreprises, profitant du développement du secteur pharmaceutique et des incitations publiques, optent pour l'acquisition de matériels et l'investissement dans l'infrastructure. Cependant, vu la complexité de fabrication pour certains produits qui nécesitent des techniques avancées et un besoin de mesures d'accompagnement, $48 \%$ des entreprises préfèrent la R\&D externe. La formation représente $40 \%$ de l'activité des entreprises car les jeunes diplômés arrivant sur le marché du travail, ont une formation inadaptée et des compétences insuffisantes. La concurrence étant rude, 
l'introduction sur le marché de nouveaux produits est liée surtout à l'innovation organisationnelle et au marketing. La figure ci desous donne un aperçu de tous ces pourcentages. En ce qui concerne la conception, l'activité interne ou la sous-traitance est chargé pour modifier la forme, l'apparence ou la facilité d'utilisation d'un bien ou service et elle est sollicitée à $24 \%$. Finalement, seul $15 \%$ des entreprises sollicite l'achat de brevets car l'incitation politique des entreprises nationales ou multinationales à produire localement est forte. La loi du 51 /49 autorise la collaboration entre les entreprises multinationales et nationales avec la possibilité de transfert technologique dont les bénéfices profitent aux deux parties.

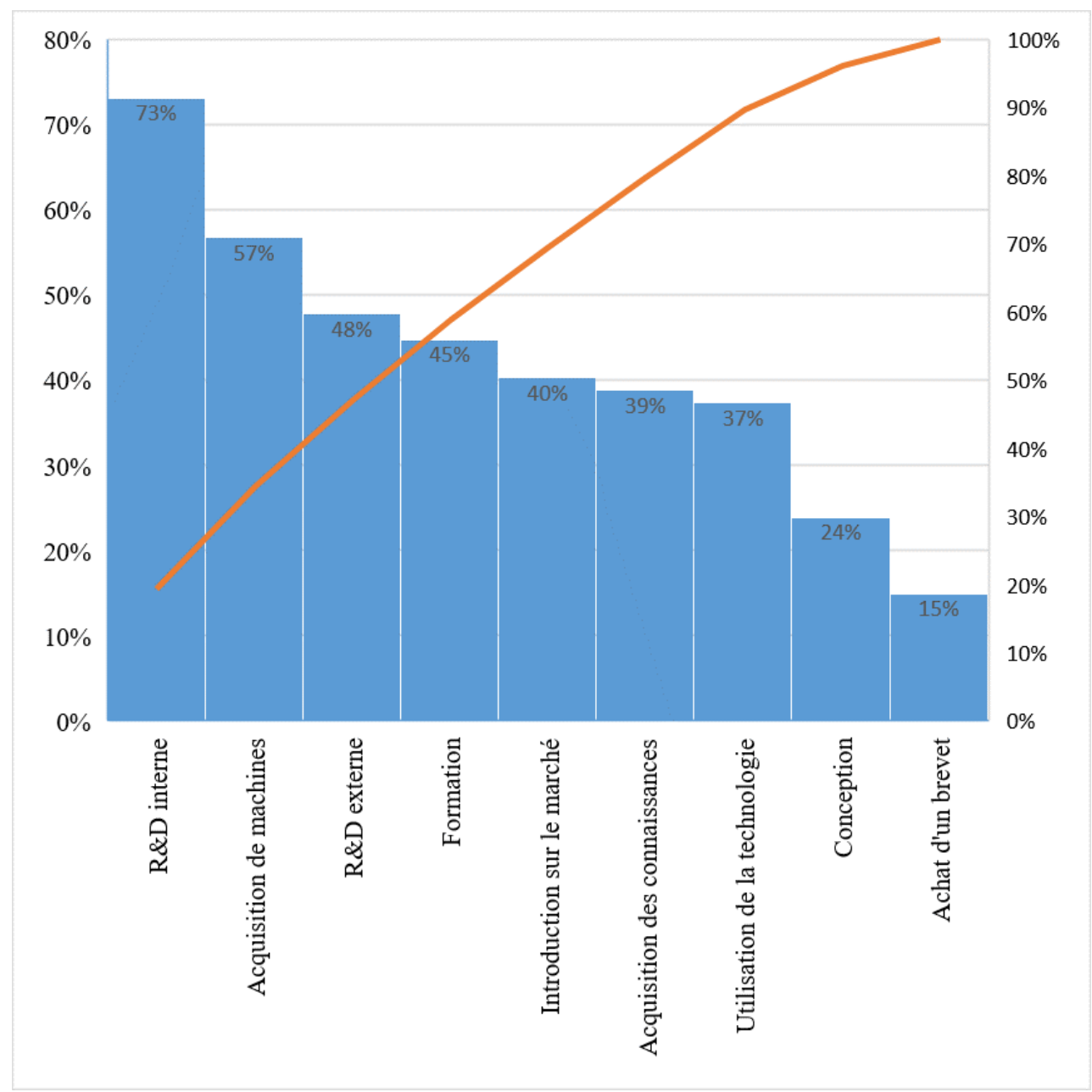

Figure 17 L'activité d'innovation des entreprises pharmaceutiques 


\subsubsection{Les obstacles à l'innovation}

Les résultats montrent (figure ci desous) le défaut de qualification des employés. $43 \%$ du personnel des entreprises est non qualifié. En conséquence, l'université doit adapter la formation aux exigences locales et à la demande des entreprises. A l'entrave à l'innovation, vient ensuite, la politique mise en place par le gouvernement pour la concurrence réglementaire avec 39\% du taux de réponses ; car le gouvernement doit assurer la régulation du marché. Le manque de financement interne pour l'innovation intervient avec $36 \%$ et également pour l'incertitude du marché vis-à-vis des idées innovantes. Les résultats de l'enquête montrent également un manque d'experts locaux sur le terrain estimé à 33\%. La production au moindre cout à cause de la concurrence dans le marché et la rentabilité du projet amènent les entreprises à produire le même produit générique compte tenu de leur manque d’expérience.

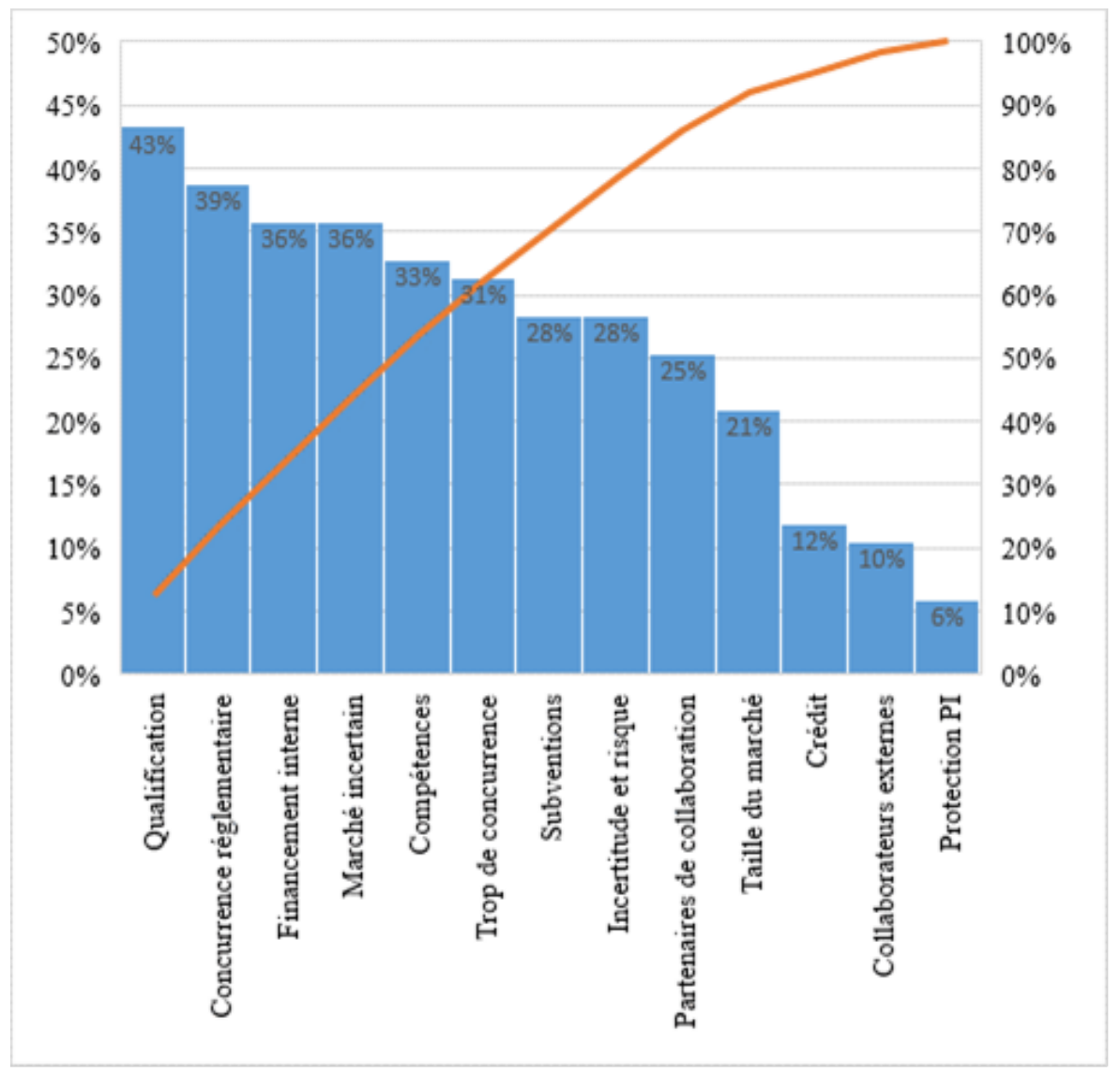

Figure 18 Les obstacles à l'innovation 


\subsubsection{Les obstacles à l'adoption de la technologie}

La démotivation des entreprises à opter pour l'acquisition d'une technologie avancée est due en premier lieu au cout élevé de la technologie avec $66 \%$ des répondants ; deuxièmement au manque de financements interne et externe et au manque d'informations sur la technologie de pointe (30\%). Ensuite c'est le manque de compétences techniques pour mener à bien la réalisation et soutenir ce type d'investissement (48\%) qui fait obstacle. En ce qui concerne la difficulté à intégrer de nouvelles technologies de pointe avec les systèmes, les normes et les processus existants, le taux est de $45 \%$. Ainsi l'entreprise n'est pas convaincue de l'avantage économique apporté par la technologie, soit $16 \%$ des répondants; $10 \%$ répondent que l'investissement n'est pas nécessaire pour la poursuite des opérations. Mis à part le financement et les compétences techniques, le fait que la culture organisationnelle est trop rigide représente $36 \%$ des répondants et le fait que l'apport de la technologie est lié avec la prise de décision par des sociétés mères, affiliées ou filiales représentent $33 \%$ (Figure ci desous)..

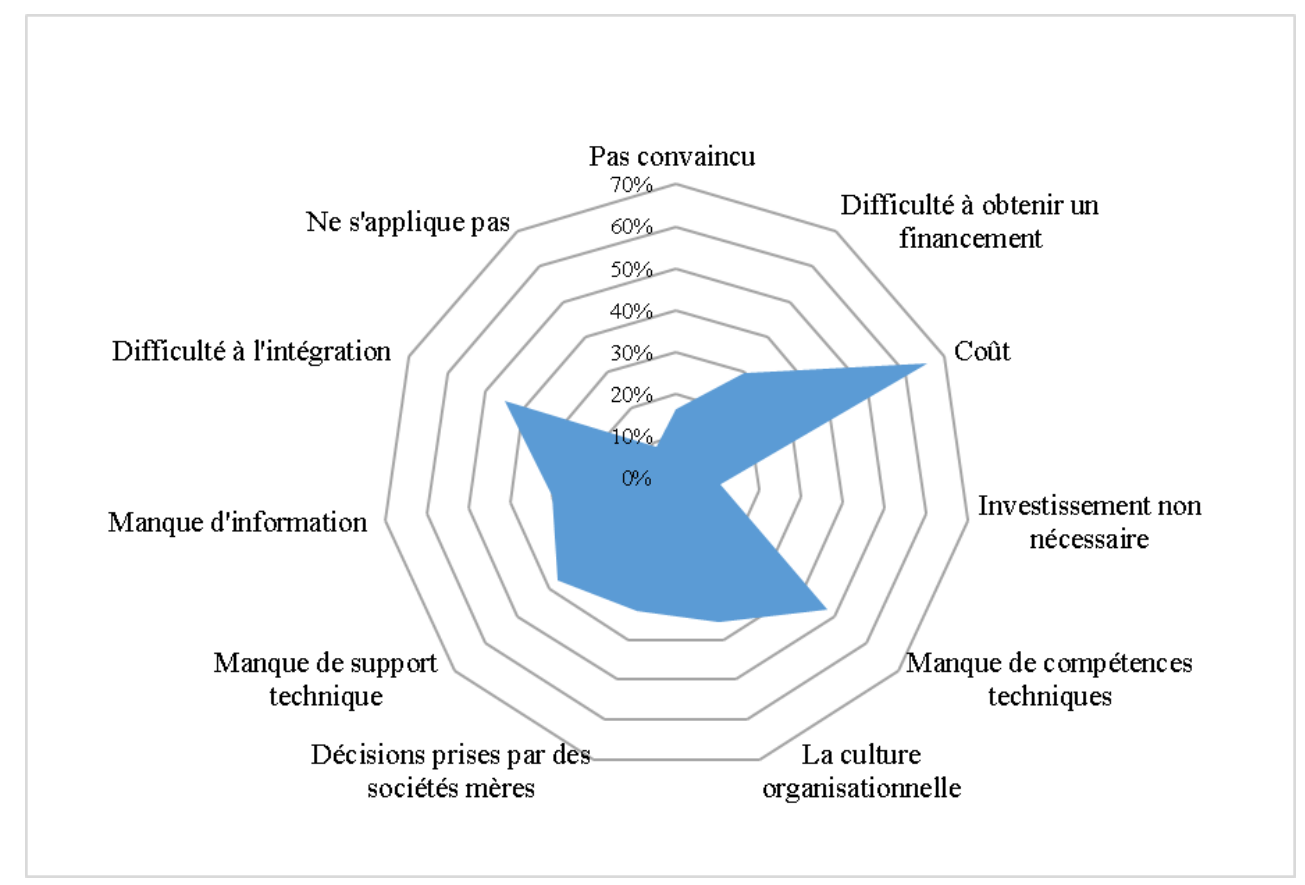

Figure 5.9 Les obstacles à l'adoption de l'innovation

\subsubsection{Conclusion}

Les perturbations économiques dues à l'instabilité et à la chute du prix du baril de pétrole ont amené les décideurs politiques algériens à revoir la politique des importations 
afin de diminuer le déficit budgétaire. L’Algérie a pris conscience de la nécessité d'instaurer une politique alternative permettant premièrement de subvenir aux besoins locaux avec la promotion de la production locale et deuxièmement à promouvoir l'innovation qui est un facteur de succès du développement des entreprises locales.

L'amélioration du niveau de vie du citoyen Algérien et les programmes d'investissement dans les infrastructures dans le secteur de la santé a accentué la demande vers les médicaments. Des instruments incitatifs ont été mis en place pour soutenir l'industrie pharmaceutique locale.

Les résultats montrent que les entreprises optent pour l'adoption des technologies à travers l'achat de matériels. Les collaborations internationales existent dans le but du transfert technologique. Un des obstacles majeurs à l'innovation et à l'intégration des technologies est sans aucun doute la qualification obsolette du personnel. Les jeunes diplômés arrivant sur le marché du travail, ont une formation inadaptée et des compétences insuffisantes ; cela constitue un obstacle non négligeable à l'absorption des technologies en entreprise. Une mise à jour de la formation dans les universités est recommandée.

A cet effet, les décisions politiques doivent être orientées vers la génération des connaissances à travers un programme de formation performant permettant à préparer les étudiants aux exigences des entreprises et à l'adoption de la technologie. Cette politique peut être traduite à travers les collaborations entre l'université et les entreprises afin d'initier les compétences et orienter les projets de recherche.

\subsection{Analyse multivariance}

Dans cette section nous avons analysé les données à l'aide du software partial least square (PLS). Dans le but d'arriver au modèle structurel, une serie de tests de validité du modèle est effectuée. Les mesures sont validées puis les hypothèses sont testées. Finalement, nous proposons le modèle structurel à l'aide de la technique Partial Least Square. Le logiciel utilisé est le programme Smart PLS3 (v.3.2.9).

\subsubsection{Fiabilité et validité des mesures}

Comme suggéré par Chin (2001), nous avons utilisé des changements d'éléments distincts pour évaluer la fiabilité d'éléments individuels. Les valeurs avec des charges supérieures à 0,7 (J. Nunnally, 1967) sont considérées comme acceptables, ce qui signifie que le sujet a expliqué environ $50 \%$ de la variance dans un élément particulier. Cette précaution garantit que les éléments de la structure de mesure mesurent le même modèle. La fiabilité composite a évalué la cohérence interne de chaque construction. Le niveau de fiabilité composite minimum acceptable est de 0,7 pour chaque chargement d'éléments (J. C. Nunnally \& Bernstein, 1994). Cette première série de résultats indique un degré élevé de fiabilité des éléments individuels (Fornell \& Larcker, 1981; Hulland, 1999) 
La méthode des moindres carrés partiels (PLS) a été utilisée pour cette analyse. Le logiciel utilisé est le programme Smart PLS3 (v.3.2.9). Les données ne doivent pas nécessairement répondre à l'exigence de normalité. C'est d'ailleurs l'une des motivations de l'utilisation du modèle PLS (J Henseler, Ringle, \& Sinkovics, 2009, pp. 277-319). Le PLS peut évaluer la cohérence et la robustesse de l'instrument. Selon Li et al. (2013), la taille de l'échantillon devrait être dix fois plus importante que le nombre le plus significatif d'indicateurs utilisés pour mesurer une seule construction (5) ou dix fois plus importante que la partie la plus substantielle d'un cheminement structurel dirigé vers une construction particulière dans le modèle. Un nombre plus important de trajectoires structurelles dirigées vers une construction particulière est de 6, et donc la taille minimale de l'échantillon serait de 60. Dans ce cas, la taille de l'échantillon utilisé dans le modèle (67) est légèrement supérieure à celle requise pour effectuer le PLS avec confiance.

Les bêtas standardisés ou coefficients de cheminement indiquent la force des associations causales entre deux variables latentes. Celles-ci sont indiquées dans la figure 5.10 .

Cohérence du modèle: Moyenne des écarts extraits (AVE). Mesure la variance saisie par une construction par rapport à la variance due à l'erreur de mesure. Il devrait être supérieur à 0,5 mais nous pouvons accepter 0,4 selon Fornell et Larcker (1981) qui proposent que si l'AVE est inférieur à 0,5 , mais que la fiabilité composite est supérieure à 0,6 , la validité convergente de la construction est toujours adéquate (voir tableau 5.1). 
Tableau 5.1 Mesures de fiabilité

\begin{tabular}{|l|l|l|l|l|}
\hline & $\alpha$ Cronbach & $\begin{array}{l}\text { Fiabilité des } \\
\text { composites }\end{array}$ & AVE & $\mathrm{R}^{2}$ \\
\hline Activités & & & & 0.397 \\
\hline Acteurs & 0.649 & 0.763 & 0.525 & \\
\hline Barrières & 0.601 & 0.770 & 0.539 & \\
\hline Propositions & 0.599 & 0.737 & 0.497 & \\
\hline Résultats & 0.603 & 0.726 & 0.480 & 0.434 \\
\hline
\end{tabular}

Tableau 5.2 Matrice de corrélation entre les variables latentes (Fornell \& Lacker, 1981)

\begin{tabular}{|c|r|r|r|r|r|}
\hline & Activités & Acteurs & Barrières & Propositions & Résultats \\
\hline Activités & & & & & \\
\hline Acteurs & 0.414 & 0.725 & & & \\
\hline Barrières & 0.346 & 0.117 & 0.734 & & \\
\hline Propositions & 0.464 & 0.322 & 0.019 & 0.690 & \\
\hline Résultats & 0.629 & 0.308 & 0.267 & 0.040 & 0.633 \\
\hline
\end{tabular}

\subsubsection{Essais des hypothèses}

Les indicateurs externes d'une variable latente réflexe sont acceptables pendant le calcul si leur coefficient est de 0,4-0,7 comme le montre la figure ci desous (Hair Jr, Hult, Ringle, \& Sarstedt, 2016). La variable latente, les activités d'innovation, est supposée être formative selon ce que Galende et de la Fuente (2003) proposent de la théorie de l'innovation, et Bollen et Lennox (1991) du point de vue statistique. Dans ce cas, l'explication maximale de la variance a été vérifiée et que les indicateurs externes sont acceptables avec le bootstrapping $(\mathrm{p}<0,05)$ et les statistiques de colinéarité (facteur d'inflation de la variance $<5$ ).

Nous avons également estimé les coefficients de régression entre les facteurs latents, leurs statistiques $t$ et les valeurs $p$, en utilisant des procédures de bootstrapping et de banderolage des yeux avec 5000 échantillons (voir tableau 5.3). Les coefficients t de 
Student se situent dans la fourchette recommandée (supérieure à 3,29 pour $\mathrm{p}<0,001$ ). Les coefficients de Stone Geiser (Q2) sont supérieurs à zéro, ce qui indique que le modèle a une pertinence prédictive positive. Le tableau 5.1 indique les mesures de fiabilité obtenues pour les variables latentes. Les valeurs de Cochran sur $\alpha$ sont au minimum acceptable de 0,6 (Jörg Henseler et al., 2014).

Tableau 5.3 Effets directs, variances expliquées et test de Stone Geiser Q2 pour des variables endogènes

\begin{tabular}{|l|l|l|l|}
\hline & $\begin{array}{l}\text { Effet direct./ indirect } \\
(\mathrm{t})\end{array}$ & $\begin{array}{l}\text { Varianza explicada } \\
(\%)\end{array}$ & $\begin{array}{l}\text { Stone Geiser } \\
\mathrm{Q}^{2}\end{array}$ \\
\hline Effets sur les résultats & $\mathrm{R}^{2}=0.434$ & & 0.161 \\
\hline Effets sur les activités & $\mathrm{R}^{2}=0.397$ & & 0.133 \\
\hline Barrières >> Activités & 3.572 & 28,37 & $* *$ \\
\hline Propositions >>> Activités & 4.933 & 44,75 & $* *$ \\
\hline Acteurs >> Activités & 3.611 & 26,88 & $* *$ \\
\hline
\end{tabular}

** $\mathrm{P}<0.001 ; * \mathrm{p}<0.05$

\subsubsection{Modèle structurel}

La figure ci desous montre les résultats du modèle structurel proposé pour l'échantillon. Les éléments observables du questionnaire sont représentés par des rectangles (uniquement ceux qui offrent des résultats significatifs) et les facteurs latents non observables par des cercles. Les coefficients de régression partielle, correspondant aux variables endogènes, sont indiqués à côté des flèches et du rapport de détermination (R2) pour les régressions correspondantes à l'intérieur des cercles représentant les valeurs latentes.

Nous pouvons observer que les résultats de l'analyse PLS indiquent ce qui suit en ce qui concerne la perception des répondants sur le système d'innovation et les politiques publiques qui le soutiennent.

L'absence d'influence des instruments politiques, ce qui implique la non-confirmation de l'hypothèse $\mathrm{H} 3$ puisque les instruments ne modèrent ni n'influencent les activités ou les résultats. De plus, les activités d'innovation influencent directement les résultats de l'innovation. En tant que modérateurs de l'influence des activités d'innovation, les obstacles à l'innovation ainsi que l'influence des agents du système d'innovation apparaissent. Les propositions d'instruments de soutien à l'innovation par les gestionnaires influencent logiquement leurs activités. Cela confirme l'hypothèse H1. Il en va de même pour le $\mathrm{H} 2$ et le $\mathrm{H} 4$ dans leur aspect d'activités modératrices. 
Les activités d'innovation qui semblent décisives sont : la R\&D interne et externe, la formation aux activités innovantes, l'introduction de produits innovants sur le marché et l'utilisation de technologies avancées.

En ce qui concerne les acteurs du système d'innovation, les universités et les centres de recherche, le gouvernement et les clients et fournisseurs se distinguent.

En tant qu'obstacles ou barrières, les résultats mettent en évidence la taille du marché, le manque de crédit, les subventions et la réglementation administrative de la concurrence sur le marché.

Enfin, parmi les propositions faites par les entreprises lors des entretiens, les suivantes sont fondamentales : Accès aux installations et infrastructures de communication, aux informations sur les marchés sectoriels et à l'assistance technique.

Les résultats les plus pertinents en matière d'innovation sont les suivants : Innovation en matière de produits et de procédés, innovation en matière d'organisation et de marketing

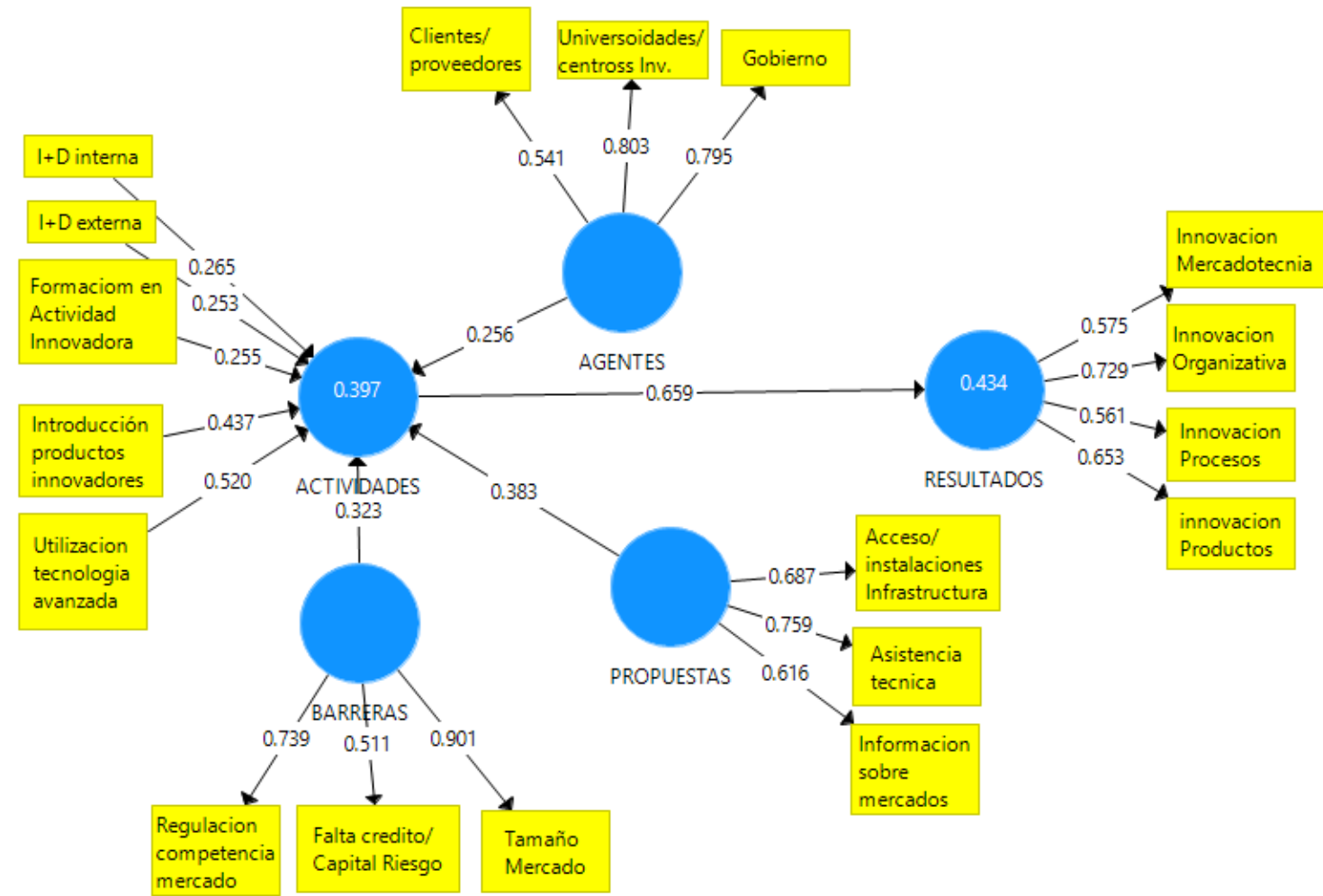

Figure 5.10 Modèle d'équations structurelles estimées à l'aide de l'échantillon 
L'objectif de cet étude est d'analyser le système des politiques d'innovation dans les pays en développement, et donc d'examiner la relation entre les acteurs de l'innovation, les instruments politiques et les activités d'innovation dans les entreprises et les performances d'innovation.

L'étude reflète la perception de certains acteurs importants, les entrepreneurs, sur le système d'innovation algérien et les politiques publiques qui le soutiennent.

Le système de politique d'innovation dans le secteur pharmaceutique algérien repose principalement sur les activités d'innovation des entreprises en termes de renforcement des capacités d'apprentissage et d'adoption des technologies, en tenant compte de la demande de R\&D interne et externe et du lancement de nouveaux produits dans ce domaine. C'est pourquoi les entreprises doivent collaborer, d'une part, avec l'université dans le cadre de la qualité de la formation. D'autre part, l'université doit adapter la formation offerte à la demande du marché.

Deuxièmement, elle doit disposer de programmes d'aménagement et d'équipement des laboratoires de recherche et des centres technologiques, indépendamment des administrations de contrôle de la qualité, des centres de recherche ou des universités. La mise à disposition de laboratoires technologiques attire l'attention des entreprises, non seulement sur les partenariats, mais aussi sur l'amélioration et le développement des produits. Sur cette base, la médiation entre les entreprises et les laboratoires de recherche publics et privés garantirait la génération de connaissances et de techniques, dans le cas du secteur pharmaceutique en Algérie.

Il est intéressant de noter que trois obstacles à l'innovation sont mis en évidence. La petite taille du marché et les difficultés de la concurrence dues aux problèmes de réglementation. Le manque de financement public et privé pour l'innovation et la formation technologique est également souligné, tout comme le besoin d'équipements et d'infrastructures de communication dans les propositions faites par les répondants.

Ces résultats ont été confirmés par les entretiens au cours desquels certains informateurs ont déclaré :

"Le système ne facilite pas la communication entre l'université et l'industrie, la première ne comprend pas nos besoins, c'est pourquoi nous ne progressons pas... nous avons développé des produits... nous avons contribué au développement de produits... et j'ai contribué à un brevet alors que je faisais partie de cette équipe de manière isolée".

"A un moment où nous avions les moyens de nous développer, l'équipe était multidisciplinaire mais difficile à maintenir".

Ils ont également exprimé leurs besoins :

"Je crois que l'innovation doit venir des entreprises car le rôle de l'État est de faciliter et de mettre en place l'environnement local pour l'innovation [...] Pour aider les 
entreprises à innover, nous devons développer les universités et les laboratoires et leur donner les moyens et, pourquoi pas, obliger les laboratoires à faire de la recherche et du développement, à créer des molécules comme ils le font à l'étranger [...] nous avons besoin d'infrastructures scientifiques et de communication [...] Nous devons former davantage de personnes dans une optique d'innovation, améliorer nos systèmes de communication [... Promouvoir les start-ups ; malheureusement, en Algérie, nous n'avons pas de culture de la start-up..."

Les résultats indiquent que la politique d'innovation s'oriente vers des actions indirectes visant à promouvoir l'innovation dans les entreprises pharmaceutiques algériennes. Il est suggéré de concentrer les efforts sur la promotion de la qualité de la formation universitaire et la réhabilitation des laboratoires de recherche (universités, institutions publiques et privées, administration publique) afin de participer au processus d'apprentissage et au soutien technique. L'éducation est un élément nécessaire dans tout système d'innovation axé sur le développement (Chaminade, Lundvall, Vang-lauridsen, \& Joseph, 2009).

L'insistance des répondants sur la taille du marché et les réglementations administratives indique un marché quelque peu autarcique et peu ouvert sur le monde extérieur. Ils sont le reflet d'un pays qui présente des traits d'isolement.

Ils améliorent également les infrastructures technologiques et de communication. À la fin de l'analyse, notre modèle permet aux décideurs politiques d'adapter les orientations de la politique d'innovation aux besoins des entreprises et des marchés en se concentrant uniquement sur les politiques en question.

Les résultats soulignent la nécessité d'adapter les politiques d'innovation au système d'innovation existant dans le pays, comme le soulignent certains auteurs de manière contingente (de Lucio et al., 2010; Charles Edquist, 2011). Les besoins du pays en matière de développement, comme l'ont souligné d'autres personnes (Chaminade et al., 2009).

\subsubsection{Conclusion}

En résumé, nous remarquons que les résultats ne montrent pas l'influence des instruments politiques ; par contre les activités et les acteurs d'innovation influencent beaucoup les résultats de l'innovation. Ces activités sont : la R\&D interne, la R\&D externe, la Formation pour des activités innovantes et l'Introduction sur le marché des innovations. Á leur tour, les activités d'innovation sont essentiellement influencées par les acteurs Universités et Gouvernement, instituts de recherche publics ou privés ainsi que par les obstacles ou barrières à l'innovation (taille du marché) et finalement, par les propositions posées par les entretiens : les programmes de formation et d'embauche, l'accès aux installations et l'assistance technique. Les résultats les plus pertinents d'innovation sont les suivants: Innovation dans le produit, Innovation dans les processus, Innovation organisationnelle et Innovation marketing. 
Evaluation de la politique d'innovation sur un échantillon d'entreprises algériennes 


\section{Chapitre 6 Conclusions générales}


Evaluation de la politique d'innovation sur un échantillon d'entreprises algériennes 


\subsection{Conclusion}

Cette thèse apporte une contribution à l'étude de l'effet de la politique d'innovation sur les résultats d'innovation dans les entreprises pharmaceutiques en Algérie. L'étude porte sur un échantillon de 67 entreprises. Elle se concentre sur la relation entre les dimensions (acteurs d'innovations, instruments politiques et activités d'innovation) et les résultats d'innovation au sein de ces entreprises. La politique d'innovation doit être adaptée et réajustée aux besoins des entreprises (Castano-Martinez, Mendez-Picazo, \& Galindo-Martin, 2015). La politique d'innovation n'est pas efficace sans l'implication pratique des acteurs. En effet l'implication pratique des acteurs contribue à l'innovation directement (S. M. Lee, Hwang, \& Choi, 2012). D'autre part l'entreprise exerce des activités qui conduisent à la génération de l'innovation. Notre étude confirme cette constatation et va plus loin en enquêtant sur la dynamique du système d'innovation en Algérie dans le secteur pharmaceutique et l'impact de la politique d'innovation sur les résultats d'innovation au sein des entreprises. Nous avons essayé de comprendre la relation entre la prise de décision politique pour l'innovation et les résultats d'innovation.

Dans ce travail nous avons essayé de répondre la question principale suivante : Quelle est la politique d'innovation appropriée pour le développement des entreprises pharmaceutiques en Algérie. Ainsi aux questions secondaires tels que : comment comprendre les aspects constructifs d'une politique d'innovation efficace ? Comment renforcer cette politique d'innovation en impliquant tous les acteurs du système d'innovation ?

Comme indiqué dans la section 2.5.4, les politiques du secteur pharmaceutique algérien ont jusqu'à présent été basées sur le soutien aux infrastructures de production, l'amélioration des conditions de fonctionnement de la production, la création d'instituts de soutien, les incitations à la fabrication, la régulation publique du marché et enfin, la centralisation des décisions politiques et administratives (qualité, prix, marques, marchés publics, etc.).

Cependant, les politiques publiques algériennes à l'égard du secteur n'ont pas abordé la question de la promotion de l'innovation ni le développement de nouveaux produits pharmaceutiques spécifiques dans le pays. En outre, le ministère de la santé exige un protocole pour la qualité des produits, mais les administrations publiques ne disposent pas des outils et des qualifications nécessaires pour faire face à l'expansion du marché des médicaments en Algérie. Troisièmement, la politique de production locale est bénéfique dans la mesure où les entreprises algériennes investissent davantage dans de nouveaux produits. Toutefois, ces entreprises n'ont pas les compétences nécessaires pour s'engager dans une production de haute technologie ou pour développer des produits à prix compétitifs ou de nouveaux produits.

Pour toutes ces raisons, les entreprises algériennes ont toujours besoin du transfert de technologies et des matières premières étrangères. À mesure que l'industrie pharmaceu- 
tique algérienne se développe, elle aura davantage besoin de subventions et de partenariats dans le domaine du développement et de l'adaptation des technologies.

Et qu'en pensent les entreprises ? Les résultats de notre étude sur le terrain indiquent ce qui suit.

(a) Tout d'abord, pour obtenir de bons résultats en matière d'innovation, il faudrait surmonter les obstacles à l'innovation. Comme obstacles à l'innovation, il faut souligner que les politiques gouvernementales en matière de concurrence et de régulation des marchés ne sont pas efficaces, que la taille du marché est très petite et qu'il faudrait donc s'attaquer aux politiques favorisant les exportations.

(b) Deuxièmement, les activités des entreprises les plus efficaces pour développer l'innovation sont le développement de la R\&D interne et externe, la formation à l'innovation et l'aide à l'introduction de nouveaux produits sur le marché.

(c) Dans le cadre de ces activités (soutien à la R\&D interne et externe), les acteurs les plus pertinents en matière d'innovation sont les universités et les instituts de recherche privés et publics.

(d) Les entreprises identifient essentiellement quatre propositions comme étant des politiques d'innovation pertinentes : les politiques fiscales pour soutenir l'innovation, les programmes de formation des employés et du personnel, les infrastructures qui facilitent l'accès aux installations et l'assistance technique (technologie, etc.).

(e) Les entreprises identifient essentiellement quatre résultats d'innovation : l'innovation dans les processus (production), les nouveaux produits, l'innovation organisationnelle et l'innovation en matière de marketing, y compris les exportations.

Que pouvons-nous en conclure ?

Tout d'abord, les travaux de terrain indiquent que, selon les entreprises pharmaceutiques algériennes, les activités des entreprises liées à l'innovation (développement de la $R \& D$ interne et externe, formation interne à l'innovation, aide à l'introduction de nouveaux produits sur le marché) ont un impact positif beaucoup plus important sur les résultats des innovations dans ce secteur que l'influence des agents/acteurs (universités et instituts de recherche publics et privés).

Par conséquent, le cas de l'Algérie ne coïncide pas avec la revue de la littérature sur l'impact direct des instruments politiques sur les résultats de l'innovation car les recommandations des chercheurs pour la politique de l'innovation dans les pays en développement sont holistiques et n'abordent pas en profondeur la question de l'efficience et de l'efficacité des politiques,(Acs \& Amorós, 2008; Dutrénit \& Puchet, 2017; Galindo \& Mendez, 2014; Navarro, Valdaliso, Aranguren, \& Magro, 2014; M. N. Sharif, 2012; Sung, 2015). 
Dans notre cas, la relation entre les acteurs et les résultats est assez faible par rapport à la relation entre les activités d'innovation et les résultats de l'innovation. Cela s'explique par l'extension du marché pharmaceutique en Algérie, suite à la baisse du prix du pétrole et du gaz, qui a entraîné une diminution des recettes de l'État. Par conséquent, la première décision politique est de réduire les coûts d'importation en proclamant une ouverture aux investissements dans la production locale et l'attraction des multinationales locales. Cela a un effet dissuasif sur la R\&D locale. En fait, la volonté d'innover et de se développer n'est présente que dans les entreprises.

Deuxièmement, les acteurs de l'innovation, notamment les universités, les instituts de recherche et le gouvernement, ont un impact sur l'activité d'innovation des entreprises. Pourquoi ? Essentiellement, parce qu'elle est la source du recrutement d'employés et de spécialistes dans le domaine de la pharmacie .

D'où la relation entre l'entreprise et le ministère de la santé dans le domaine de la réglementation du marché et des procédures d'achat ou de vente ou de contrôle des médicaments. D'autre part, le manque de partenaires en collaboration affecte les niveaux de compétence et la diffusion des connaissances pour l'innovation (Kang \& Park, 2012).

En outre, l'attrait des collaborateurs externes et la régénération de l'intérêt commun (Yun \& Lee, 2013) basée sur la triple vision de Helics (relation entre l'université, l'industrie et le gouvernement) devraient affecter les résultats de l'innovation (Etzkowitz \& Leydesdorff, 2000; Leydesdorff, 2000). Ce n'est malheureusement pas le cas des résultats que nous avons obtenus suite à l'étude sur le terrain.

Nos résultats montrent une relation directe entre les acteurs et l'activité d'innovation. Cette relation reste cependant limitée au gouvernement, aux instituts de recherche publics ou privés et aux universités sans véritable transfert de connaissances entre eux. Elle est traduite dans le cas algérien par un investissement dans les infrastructures plutôt que dans la qualité (Djeflat, 2010) afin d'augmenter le nombre de nouveaux diplômés universitaires et ainsi accroître les possibilités de absorbé la demande du marché du travail dans des domaines specefique

Troisièmement, les entreprises pharmaceutiques algériennes ont opté pour des activités d'innovation en utilisant la R\&D interne et externe, la formation aux activités d'innovation et l'introduction des innovations sur le marché. Ces activités permettent à l'entreprise de réaliser des innovations se rapportant à : l'innovation du produit, l'innovation du processus de fabrication, l'innovation organisationnelle et l'innovation marketing. En ce qui concerne l'activité de R\&D interne, les entreprises développent le produit localement et avec des moyens limités à leur portée. Certaines entreprises préfèrent investir dans la formation pour pouvoir développer le produit et d'autres préfèrent engager des spécialistes ou collaborer avec des multinationales pour le transfert de technologie. En ce sens, une analyse détaillée de la question de la politique d'innovation axée sur la demande est recommandée (D’Este et al., 2012). 
Quatrièmement, les entreprises pharmaceutiques sont confrontées à des obstacles à l'activité d'innovation en raison de la taille du marché et de la politique réglementaire ou gouvernementale en matière de concurrence mise en oeuvre. Ces deux obstacles sont gérés par le ministère de la santé ou le ministère de l'industrie et des mines dans le cas de l'Algérie.

Dans cette hypothèse, l'analyse documentaire confirme nos conclusions sur les obstacles à l'innovation, mais ne confirme pas l'impact des obstacles sur les activités d'innovation. Ainsi, Baumol (2004) explique la relation entre la croissance et les performances en matière d'innovation dans les économies de marché libre. En outre, Cozzi (2007) explique que dans les pays en développement, les marchés parfaits permettent aux monopoles de tenir le coup malgré les obstacles vis a vis la concurrence. Ainsi, Thorsteindottir (2007) a examiné le rôle des systèmes de santé dans l'innovation en matière de biotechnologie au Brésil, à Cuba et en Inde. Elle a identifié les façons avec lesquelles les systèmes de santé locaux affectent le processus d'innovation. Par la suite, Allard et al. (2012) ont examiné les interactions complexes entre l'innovation technologique, les marchés, la réglementation et la société civile. En outre, Zhou et Minshall (2014) constatent que les barrières institutionnelles sont réelles et demeurent le maillon faible de la relation entre l'industrie et l'écosystème.

Dans le cas de l'Algérie, cet obstacle administratif est dû à l'expansion massive du secteur pharmaceutique, à l'absence de culture administrative, à la faiblesse du lien entre l'administration et l'entreprise, au manque d'information et d'analyse lors de la prise de décision et enfin, au fait que les travailleurs n'ont pas de formation technologique suffisante dans le domaine de la pharmacie.

Cinquièmement, les entreprises proposent un support direct à l'activité de l'innovation qui consiste en Crédit d'impôt du gouvernement pour la recherche et le développement ou en dépenses pour l'immobilier, les programmes de formation et d'embauche, l'accès aux installations et l'assistance technique.

L'absence de crédit privé pour les activités d'innovation est expliquée) par le manque de confiance entre la demande et la banque (Sedkaoui, 2016). Par conséquent, Bae et Yoo (2015) montrent que les politiques visant à promouvoir l'innovation dans les industries créatives devraient réduire ce niveau de méfiance vis á vis des banques dans les pays en développement. Nos travaux montrent que les entreprises pharmaceutiques algériennes ont besoin d'un soutien politique direct à l'activité plutôt que d'une motivation à l'innovation.

Concernant l'appui à la formation, il est pris en compte dans nos résultats à plusieurs reprises et sous diverses formes. En effet, l'appui technique de la part des administrations publiques ainsi que l'accès aux installations, exige des compétences spécifiques et adaptées à la technologie appropriée à ces entreprises. Donc si l'administration algérienne n'arrive pas à faire face aux exigences technologiques, comme doter les laboratoires de contrôle ou d'analyse de matériels performants ainsi que préparer et former 
les gens qui travaillent dans ces laboratoires, dument la politique d'innovation publique ne pourra pas participer à la promotion de l'innovation au sein des entreprises $(\mathrm{H}$. Gupta \& Barua, 2016; Kuhlmann \& Ordóñez-Matamoros, 2017; Wong \& Goh, 2015).

Finalement, le système en Algérie ne permet pas la génération des connaissances étant donné qu'il n’y a pas une relation entre les résultats d'innovations et les acteurs d'innovations et également avec l'activité d'innovation. Selon, Fernandez et al (2010) La politique du système d'innovation est basée sur trois générations, que lui explique à travers une analyse des projets régionaux en Espagne. La première génération est basée sur la création des connaissances nouvelles à partir d'un processus d'innovation linéaire de la $R \& D$ et à l'expérimentation qui se concentre sur l'offre ; à ce niveau la politique de la promotion de la recherche scientifique et de la R\&D prime. La deuxième génération met l'accent sur les instruments de régulation, organisation et institution comme la fiscalité, la propriété intellectuelle. Finalement, la troisième génération se focalise sur un système plus complexe où la création des connaissances et la promotion de la génération des connaissances est importante à cette étape.

A cet effet, on peut dire que le cas du secteur pharmaceutique en Algérie est en première phase. Cette phase est caractérisée par l'identification des acteurs clefs et leur implication dans le processus d'innovation. Dans le cas du secteur pharmaceutique Algérien, l'implication des acteurs clefs signifie : premièrement l'université doit revoir son système de formation du point de vu qualité et création de formations adaptées aux besoins des entreprises et aux exigences du marché. Deuxièmement, revoir les conditions d'apprentissage au sein des laboratoires de recherche. Les jeunes sortants des universités vont par conséquent être absorbés par le marché du travail ; une partie d'entre eux va occuper des postes aux centres de recherche publique ou privé ou bien dans les administrations publiques. S'ils ne pas aptes ou pas suffisamment préparés aux exigences du marché, les conséquences seront lourdes pour les entreprises en ce qui concerne les dépenses pour la formation ainsi que la connexion entre l'administration et l'entreprise en matière de suivi technique, de consulting et de prise de décision.

\subsection{Implication et recommandation pour la politique d'innovation dans les pays en développement}

Nos implications futures sont : Premièrement, approfondir plus sur les motivations d'innovation et les principaux obstacles à l'activité d'innovation, en utilisant la méthodologie qualitative à partir des interviews, dans le secteur pharmaceutique en Algérie. Cette méthode va permettre de découvrir d'autres facteurs qui n'ont pas été cités dans l'enquête de Canadienne de 2017 «Integrated Business Statistics Program (IBSP) Survey of Innovation and Business Strategy » et du questionnaire «The Community Innovation Survey» (CIS) du 2014. Deuxièmement, appliquer notre modèle dans les autres secteurs d'activité en Algérie. Cette proposition va permettre d'avoir une vision large sur les politiques d'innovation globales, car nous supposons que l'interaction entre les acteurs diffère d'un secteur à un autre (Pavitt, 1984). Troisièmement, appliquer le mo- 
dèle dans les pays émergents et le comparer avec les pays en voie de développement. Notre modèle, ne se limite pas seulement à la problématique de la politique d'innovation ; on peut aussi l'utiliser dans d'autres circonstances comme à la problématique du développement durable en réajustant les instruments politiques et l'activité en question. It was found that the same factors (investing in R\&D at home, formal training) were the significant determinants of product, process, organisational, marketing or logistic innovation in the case of Tunisian SME's. Thus, the policy implications of the results recommend that firms should make significant factors as the priorities to boost innovation (Bourouaha \& Maliki, 2021).

\subsection{Etendue et limitation de l'étude}

Ce travail de recherche concerne la compréhension du système politique d'innovation et la recherche des facteurs clefs qui déterminent l'innovation au sein des entreprises en examinant la relation entre les acteurs de l'innovation, les instruments politiques et les activités d'innovation au sein des entreprises avec les résultats d'innovations. L'étude ne se limite pas seulement à l'étude des principales dimensions mais va au dela en détectant le facteur clef de chaque composante. Nous avons appliqué les techniques PLS pour tester le modèle théorique à l'aide d'un questionnaire inspiré de l'enquête du Canadienne de 2017 «Integrated Business Statistics Program (IBSP) Survey of Innovation and Business Strategy» et du questionnaire «The Community Innovation Survey» (CIS) du 2014. Nous avons analysé notre échantillon à partir des réponses de 67 entreprises pharmaceutiques Algériennes. Nos résultats confirment la relation entre activités d'innovation et acteurs d'innovation avec les résultats d'innovations. En revanche l'étude ne soutient pas la relation entre instruments politiques et résultats d'innovation ni entre les autres dimensions.

En effet le système politique d'innovation dans le secteur pharmaceutique algérien est axé sur l'activité d'innovation des entreprises en matière de renforcement des capacités d'apprentissage et l'adoption des technologies par la R\&D interne et externe et le lancement de nouveaux produits sur le terrain. Donc, l'entreprise a besoin de collaborer premièrement, avec l'université sur le sujet de l'amélioration de la qualité de la formation ainsi que sur son adaptation à la demande du marché du travail. Deuxièmement, investir sur les laboratoires de recherche et les centres technologiques que se soient les administrations de contrôle de qualité, les centres de recherche ou bien les universités. La dotation des laboratoires en moyens technologiques attire l'attention des entreprises non seulement vers des collaborations mais aussi vers l'amélioration du produit et son développement. De là, la médiation entre les entreprises, les laboratoires de recherche publics et privés assure la génération du savoir et du savoir-faire dans le cas du secteur pharmaceutique Algérien.

Cette étude a quelques limites. Premièrement, les limites sont de caractère méthodologique. L'utilisation de la méthode (PLS) dans notre étude a des limites dans la taille de 
l'échantillon et les variables de contrôle (Balambo \& Baz, 2014; Goodhue, Lewis, \& Thompson, 2006; Lindberg, Persson, \& Wold, 1983; Marcoulides \& Saunders, 2006). La technique prend en considération seulement les composants statiquement significatifs et rejette les autres composants (Hung, 2017; Li, Liao, \& Yen, 2013; Nambisan, S., 2013; Radosevic \& Yoruk, 2012; Talke, Salomo, \& Rost, 2010; Yam, Lo, Tang, \& Lau, 2011).

Deuxièmement, notre cadre théorique a des limites puisqu'il se base sur la revue de littératures portant sur la politique d'innovation appliquée au pays émergents ou pays en développement en général mais pas spécifiquement au cas de la politique d'innovation dans le secteur pharmaceutique. Par ailleurs, notre cadre théorique est constitué sur la base des facteurs qui déterminent chaque dimension en question, donc chaque cas peut surgir différemment. Troisièmement, on ne peut pas généraliser nos résultats sur la politique d'innovation en Algérie ni dans les pays en développement vu la taille de l'échantillon considéré et la spécificité du secteur.

Nos résultats impliquent une orientation politique d'innovation vers des actions indirectes dans le cadre de la promotion de l'innovation au sein des entreprises Algériennes pharmaceutiques. Nous suggérons de focaliser les efforts vers la promotion de la qualité de la formation universitaire et la réhabilitation des laboratoires de recherche (Universités, institutions publiques et privées, administration publique) afin de participer au processus d'apprentissage et d'accompagnement techniques. Au terme de l'analyse, notre modèle permet aux décideurs politiques d'adapter les orientations politiques d'innovations aux exigences des entreprises et au marché. 


\section{Bibliographie}




\subsection{Bibliographie}

Abate, G. T., Rashid, S., Borzaga, C., \& Getnet, K. (2016). Rural Finance and Agricultural Technology Adoption in Ethiopia: Does the Institutional Design of Lending Organizations Matter? World Development, 84, 235-253. https://doi.org/10.1016/j.worlddev.2016.03.003

Abdelhammid, B., \& Samir Baha Eddine, M. (2019). Roa Iktissadia Review Determinants of Firm's Innovation in the MENA Region: a descriptive Analysis Determinants of Firm's Innovation in the MENA Region: a descriptive Analysis (PP 77-89).

Acs, Z., \& Amorós, J. (2008). Entrepreneurship and competitiveness dynamics in Latin America. Small Business Economics, 31(3), 305-322. https://doi.org/10.1007/slll87-008-9133-y

Adner, R. (2006). Match Your Innovation Strategy to Your Innovation Ecosystem. Retrieved from www.hbrreprints.org

Afuah, A. N., \& Utterback, J. M. (1997). Responding to structural industry changes: A technological evolution perspective. Industrial and Corporate Change. https://doi.org/10.1093/icc/6.1.183

Allard, G., Martinez, C. A., \& Williams, C. (2012). Political instability, pro-business market reforms and their impacts on national systems of innovation. Research Policy, 41(3), 638651. https://doi.org/10.1016/j.respol.2011.12.005

Amdaoud, M. (2017). Le Système National d’Innovation en Algérie: entre inertie institutionnelle et sous-apprentissage. Innovations, (2), 69-104.

Andersen, A. D. (2012a). Innovation systems and natural resources - The case of sugarcane in Brazil. Innovation and Development, 2(1), 194-195. https://doi.org/10.1080/2157930X.2012.663589

Andersen, A. D. (2012b). Towards a new approach to natural resources and development: the role of learning, innovation and linkage dynamics. International Journal of Technological Learning, Innovation and Development, 291. https://doi.org/10.1504/IJTLID.2012.047681 
Andersen, E. S., \& Lundvall, B.-Å. Å. (1997). National Innovation Systems and the Dynamics of the Division of Labor. Systems of Innovation: Technologies, Institutions and Organizations, 242-265. Retrieved from https://www.researchgate.net/profile/Charles_Edquist/publication/228315614_Systems_of_I nnovation_Technologies_Institutions_and_Organizations/links/5580106d08aec87640df220f/ Systems-of-Innovation-Technologies-Institutions-and-Organizations.pdf\#page=256

Archibugi, D., \& Pietrobelli, C. (2003). The globalisation of technology and its implications for developing countries Windows of opportunity or further burden? Technological Forecasting and Social Change, 70(9), 861-883. https://doi.org/10.1016/S0040-1625(02)00409-2

Arocena, R., \& Sutz, J. (2010). Weak knowledge demand in the South: Learning divides and innovation policies. Science and Public Policy, 37(8), 571-582. https://doi.org/10.3152/030234210X12767691861137

Arocena, R., \& Sutz, J. (2017). Science, technology and innovation for what? Exploring the democratization of knowledge as an answer. In Research Handbook on Innovation Governance for Emerging Economies (pp. 377-404). Edward Elgar Publishing. Retrieved from https://ideas.repec.org/h/elg/eechap/15643_13.html

Avila, R. C., Bernal, E., Adoracion, J., Moral, M., \& Poyatos, R. P. (2014). Management Decision Improving e-economy by regional governments. Management Decision Management Decision Iss Management Decision, 52(3), 559-572. Retrieved from http://dx.doi.org/10.1108/MD-08-2012-0589

Bae, S. H., \& Yoo, K. (2015). Economic modeling of innovation in the creative industries and its implications. Technological Forecasting and Social Change, 96, 101-110. https://doi.org/10.1016/j.techfore.2015.02.010

Barber, J. (1979). Economic sanctions as a policy instrument. International Affairs (Royal Institute of International Affairs 1944-), 55(3), 367-384.

Barre, R. (1996). Relationships between multinational firms' technology strategies and national innovation systems: A model and an empirical analysis. Innovation, Patents and 
Evaluation de la politique d'innovation sur un échantillon d'entreprises algériennes

Technological Strategies, 201-222.

Bartels, F. L., Voss, H., Lederer, S., \& Bachtrog, C. (2012). Determinants of National Innovation Systems: Policy implications for developing countries. Innovation-Management Policy \& Practice, 14(1), 2-18.

Baumol, W. J. (2004). Entrepreneurial enterprises, large established firms and other components of the free-market growth machine. Small Business Economics. https://doi.org/10.1023/B:SBEJ.0000026057.47641.a6

Becker, W., \& Dietz, J. (2004). R\&D cooperation and innovation activities of firms-evidence for the German manufacturing industry. Research Policy, 33(2), 209-223.

Bell, M., \& Figueiredo, P. N. (2012). Innovation capability building and learning mechanisms in latecomer firms: recent empirical contributions and implications for research. Canadian Journal of Development Studies/Revue Canadienne d'études Du Développement, 33(1), 14 40. https://doi.org/10.1080/02255189.2012.677168

Bemelmans-Videc, M. L., Rist, R. C., \& Vedung, E. (2010). Carrots, Sticks and Sermons: Policy Instruments and Their Evaluation. In Comparative Policy Evaluation Series (p. 277).

Bemelmans-Videc, M., Rist, R., \& Vedung, E. (2011). Carrots, sticks, and sermons: Policy instruments and their evaluation.

Bergek, A., Hekkert, M., \& Jacobsson, S. (2008). Functions in innovation systems: A framework for analysing energy system dynamics and identifying goals for system-building activities by entrepreneurs and policy makers. Innovation for a Low Carbon Economy: Economic, Institutional and Management Approaches, 79.

Bergman, E. M., Maier, G., \& Tödtling, F. (1991). Regions reconsidered: Economic networks, innovation, and local development in industrialized countries. Mansell. Mansell.

Bessant, J. R., \& Venables, T. (2008). Creating wealth from knowledge: meeting the innovation challenge. Edward Elgar.

Bhatti, Y. A. (2012). What is Frugal, What is Innovation? Towards a Theory of Frugal Innovation. 
Bibliographie

SSRN Electronic Journal, 1-45. https://doi.org/10.2139/ssrn.2005910

Blind, K. (2016). 15. The impact of regulation on innovation. Handbook of Innovation Policy Impact, 450.

Boekholt, P. (2010). The evolution of innovation paradigms and their influence on research, technological development and innovation policy instruments. In The Theory and Practice of Innovation Policy (Edward Elg). Edward Elgar Publishing.

Bollen, K., \& Lennox, R. (1991). Conventional wisdom on measurement: A structural equation perspective. Psychological Bulletin, 110(2), 305.

Bonnemain, B. (2009). Histoire de la pharmacie française en Algérie (1830-1962). Revue d'histoire de La Pharmacie, 96(363), 303-326. https://doi.org/10.3406/pharm.2009.22077

Borrás, S., \& Edquist, C. (2013). The choice of innovation policy instruments. Technological Forecasting $\quad$ and $\quad$ Social 1513-1522. https://doi.org/10.1016/j.techfore.2013.03.002

Borrás, S., \& Laatsit, M. (2019). Towards system oriented innovation policy evaluation? Evidence from EU28 member states. Research Policy, 48(1), 312-321. https://doi.org/10.1016/J.RESPOL.2018.08.020

Bourouaha, A., \& Maliki, S.B. (2021). Determinants of Firms Innovation and the role of R\&D Investment and Training: An Empirical evidence from Tunisian SME's, International Journal of Business Innovation and Research, DOI: 10.1504/IJBIR.2020.10026171, (Forthcoming)

Braczyk, H.-J., Cooke, P., \& Heidenreich, M. (1998). Regional Innovation Systems: The Role of Governances in a Globalized World. UCL Press.

Brandt, L., Thun, E., Thunb, E., \& Thun, E. (2016). Constructing a Ladder for Growth: Policy, Markets, and Industrial Upgrading in China. World Development, 80, 78-95. https://doi.org/10.1016/j.worlddev.2015.11.001

Breschi, S. (1999). Spatial patterns of innovation: evidence from patent data. The Organization of Economic Innovation in Europe, 1999, 71-102. 
Breschi, S., \& Malerba, F. (1997). Sectoral Innovation Systems: Technological Regimes, Schumpeterian Dynamics, and Spatial Boundaries. In Systems of innovation: Technologies, institutions and organizations (pp. 130-156). https://doi.org/10.1016/S0024-6301(98)902448

Brightman, R. (1939). The Social Function of Science. Nature, 143(3616), 262-263. https://doi.org/10.1038/143262a0

Brinson, M. M., Lugo, A. E., \& Brown, S. (1981). Primary Productivity, Decomposition and Consumer Activity in Freshwater Wetlands. Annual Review of Ecology and Systematics, 12(1), 123-161. https://doi.org/10.1146/annurev.es.12.110181.001011

Bush, V. (1945). The Endless Frontier, Report to the President on a Program for Postwar Scientific Research. Retrieved from http://www.dtic.mil/get-tr-doc/pdf?AD=ADA361303

Bush, Vannevar, \& Bush, V. (1945). As we may think. Resonance, 5(11).

Butler, I., Galassi, G., \& Ruffo, H. (2016). Public funding for startups in Argentina: an impact evaluation. Small Business Economics, 46(2), 295-309. https://doi.org/10.1007/s11187-0159684-7

Caniëls, M. C. J., \& Romijn, H. A. (2008). Strategic niche management: Towards a policy tool for sustainable development. Technology Analysis and Strategic Management, 20(2), 245-266. https://doi.org/10.1080/09537320701711264

Capriati, M. (2017). Capabilities, innovation and economic growth: Policymaking for freedom and efficiency. Capabilities, Innovation and Economic Growth: Policymaking for Freedom and Efficiency. Taylor and Francis. https://doi.org/10.4324/9781315718415

Carayannis, E., \& Grigoroudis, E. (2014). Linking innovation, productivity, and competitiveness: implications for policy and practice. Journal of Technology Transfer, 39(2), 199-218. https://doi.org/10.1007/s10961-012-9295-2

Carlsson, B., \& Stankiewicz, R. (1995). On the Nature, Function and Composition of Technological Systems. In Technological Systems and Economic Performance: The Case of Factory Automation (pp. 21-56). Springer. 
Casadella, V., \& Younes Bouacida, R. (2020). The primacy of innovation capacities in the NIS of the Maghreb countries: An analysis in terms of learning capacity in Morocco, Tunisia and Algeria. African Journal of Science, Technology, Innovation and Development, 12(2), 231242. https://doi.org/10.1080/20421338.2019.1633097

Casper, S., \& van Waarden, F. (2005). Innovation and institutions: A multidisciplinary review of the study of innovation systems. Edward Elgar Publishing.

Castano-Martinez, M. S., Mendez-Picazo, M. T., \& Galindo-Martin, M. A. (2015). Policies to promote entrepreneurial activity and economic performance. Management Decision, 53(9), 2073-2087. https://doi.org/10.1108/md-06-2014-0393

Chaminade, C., Lundvall, B.-Å., Vang-lauridsen, J., \& Joseph, K. (2009). Innovation policies for development: towards a systematic experimentation-based approach. October, (October), 68.

Chen, C. J., Wu, H. L., \& Lin, B. W. (2006). Evaluating the development of high-tech industries: Taiwan's science park. Technological Forecasting and Social Change, 73(4), 452-465. https://doi.org/10.1016/j.techfore.2005.04.003

Chen, D., Karami, A., Chen, D., \& Karami, A. (2010). Critical success factors for inter-firm technological cooperation: an empirical study of high-tech SMEs in China) 'Critical success factors for inter-firm technological cooperation: an empirical study of high-tech SMEs in China. Int. J. Technology Management, 513434(2), 282-299. https://doi.org/10.1504/IJTM.2010.033806

Chen, J. (2002). The challenge of R\&D globalization and the management task. Management Review (Chinese Journal, 11(11), 50-55.

Chesbrough, H. (2003). Open Innovation: The New Imperative for Creating And Profiting from Technology. Publisher: Harvard Business School Press . Retrieved from http://ictlogy.net/bibliography/reports/projects.php?idp=2546\&lang=es

Chesbrough, H. W. (2003). Open innovation : the new imperative for creating and profiting from technology. Harvard Business School Press. 
Chesbrough, H. W., \& Crowther, A. K. (2006). Beyond high-tech: early adopters of Open Innovation in other industries. $R \& D$ Management, 36(3), 229-236. https://doi.org/10.1111/j.1467-9310.2006.00428.x

Chin, W. W. (1998). The partial least squares approach to structural equation modeling. Modern Methods for Business Research, 295(2), 295-336.

Chin, W. W. (2001). PLS-Graph User's Guide Version 3.0. Retrieved from http://www.spsspasw.ir/upload/images/ei8gx66re11tenmq0sm.pdf

Cho, K., Kim, C., \& Shin, J. (2015). Differential effects of intellectual property rights on innovation and economic performance: A cross-industry investigation. Science and Public Policy, 42(6), 827-840. https://doi.org/10.1093/scipol/scv009

Choi, S. B., \& Williams, C. (2013). Innovation and firm performance in Korea and China: a crosscontext test of mainstream theories. Technology Analysis \& Strategic Management, 25(4), 423-444. https://doi.org/10.1080/09537325.2013.774346

CHOUAL, I. E. (2016). Les PME dans le marché algérien du médicament, état des lieux et perspectives. Recherches Économiques Asjp.Cerist.Dz, 11(no 14), 34-58. Retrieved from https://www.asjp.cerist.dz/en/article/23933

Christensen, C. M. (1997). The Innovator's Dilemma. Business, 1-179. https://doi.org/10.1515/9783110215519.82

Churchill Jr, G. A. (1979). A paradigm for developing better measures of marketing constructs. Journal of Marketing Research, 16(1), 64-73.

Cohen, W. M., \& Levinthal, D. A. (1990). Absorptive Capacity: A New Perspective on Learning and Innovation. Administrative Science Quarterly, 35(1), 128. https://doi.org/10.2307/2393553

Collecting, G. F. O. R., \& Data, I. I. (2005). Third edition ORGANISATION FOR ECONOMIC CO-OPERATION. Communities (Vol. Third edit). Organisation for Economic Co-operation and Development. https://doi.org/10.1787/9789264013100-en 
Cooke, P. (2004). Introduction: Regional innovation systems - an evolutionary approach. Regional Innovation Systems: The Role of Governance in a Globalized World, 1-18.

Cooke, Philip, Gomez U, M., Etxebarria, G., \& U, M. G. (1997). Regional innovation systems: Institutional and organisational dimensions. Research Policy, 26(4-5), 475-491. https://doi.org/10.1016/S0048-7333(97)00025-5

Cooke, Philip, Gomez Uranga, M., \& Etxebarria, G. (1997). Regional innovation systems: Institutional and organisational dimensions. Research Policy, 26(4-5), 475-491. https://doi.org/10.1016/S0048-7333(97)00025-5

Cooke, Philip, \& Uranga, M. G. (1997). dimensions. Research Policy, 26(4-5), 475-491. https://doi.org/10.1016/S0048-7333(97)00025-5

Cooke, PN, Heidenreich, M., \& Braczyk, H. (2004). Regional Innovation Systems: The role of governance in a globalized world.

Coombs, R., \& Hull, R. (1998). “Knowledge management practices” and path-dependency in innovation. Research Policy, 27(3), 239-256.

Cozzi, G. (2007). Self-fulfilling prophecies in the quality ladders economy. Journal of Development Economics, 84(1), 445-464. https://doi.org/10.1016/j.jdeveco.2005.12.004

Creswell, J. W. (2009). Mapping the field of mixed methods research. Sage Publications Sage CA: Los Angeles, CA.

Criscuolo, C., Haskel, J. E., \& Slaughter, M. J. (2010). Global engagement and the innovation activities of firms. International Journal of Industrial Organization, 28(2), 191-202. https://doi.org/10.1016/j.ijindorg.2009.07.012

Criscuolo, P. (2006). The "home advantage" effect and patent families. A comparison of OECD triadic patents, the USPTO and the EPO. In Scientometrics (Vol. 66, pp. 23-41). https://doi.org/10.1007/s11192-006-0003-6

Cronbach, L. J. (1951). Coefficient alpha and the internal structure of tests. Psychometrika, 16(3), 297-334. 
Crosby, L. A., Bitner, M. J., \& Gill, J. D. (1990). Organizational structure of values. Journal of Business Research, 20(2), 123-134. https://doi.org/10.1016/0148-2963(90)90056-J

Crotty, M. (1998). The foundations of social research: meaning and perspective in the research process. Sage Publications.

Cunningham, P., \& Gök, A. (2016). The impact of innovation policy schemes for collaboration. Handbook of Innovation Policy Impact, 239.

Cunningham, P, Gok, A., \& Laredo, P. (2012). The Impact of Direct Support to R\&D and Innovation in Firms: Compendium of Evidence on the Effectiveness of Innovation Policy Intervention. Manchester Institute of Innovation Research, University of Manchester.

Cunningham, Paul, Gök, A., \& Larédo, P. (2016). The impact of direct support to R\&D and innovation in firms. Handbook of Innovation Policy Impact, 54-107.

Cunningham, Paul, \& Ramlogan, R. (2016). The impact of innovation networks. Handbook of Innovation Policy Impact, 279-317.

D’Este, P., Iammarino, S., Savona, M., \& von Tunzelmann, N. (2012). What hampers innovation? Revealed barriers versus deterring barriers. Research Policy, 41(2), 482-488. https://doi.org/10.1016/J.RESPOL.2011.09.008

Da Silveira, G. (2001). Innovation diffusion: Research agenda for developing economies. Technovation. https://doi.org/10.1016/S0166-4972(01)00007-4

Damanpour, F. (1991). ORGANIZATIONAL INNOVATION: A META-ANALYSIS OF EFFECTS OF DETERMINANTS AND MODERATORS. Academy of Management Journal, 34(3), 555-590. https://doi.org/10.2307/256406

Dantas, E., \& Bell, M. (2011). The Co-Evolution of Firm-Centered Knowledge Networks and Capabilities in Late Industrializing Countries: The Case of Petrobras in the Offshore Oil Innovation System in Brazil. World Development, 39(9), 1570-1591. https://doi.org/10.1016/j.worlddev.2011.02.002

Danzon, P. M., \& Towse, A. (2003). Differential Pricing for Pharmaceuticals: Reconciling Access, 
R\&amp;D and Patents. International Journal of Health Care Finance and Economics, 3(3), 183-205. https://doi.org/10.1023/A:1025384819575

de Lucio, I. F., Mas-Verdu, F., \& Tortosa, E. (2010). Regional innovation policies: The persistence of the linear model in Spain. Service Industries Journal, 30(5), 749-762. https://doi.org/10.1080/02642060802398093

De Mattos, C., Burgess, T. F., \& Shaw, N. E. (2013). The impact of R\&D-specific factors on the attractiveness of small- and medium-sized enterprises as partners vis-a-vis alliance formation in large emerging economies. $R \quad \& \quad D$ Management, 43(1), 1-20. https://doi.org/10.1111/j.1467-9310.2012.00699.x

Delvenne, P., \& Thoreau, F. (2017). Dancing without listening to the music: learning from some failures of the 'national innovation systems' in Latin America. Research Handbook on Innovation Governance for Emerging Economies, 37-59.

Deniozos, D. (1994). Steps for the introduction of technology management in developing economies: the role of public governments. Technovation, 14(3), 197-203. https://doi.org/10.1016/0166-4972(94)90056-6

Di Vita, G. (2013). The TRIPs agreement and technological innovation. Journal of Policy Modeling, 35(6), 964-977. https://doi.org/10.1016/j.jpolmod.2013.02.001

Diez, M. A. (2001). The evaluation of regional innovation and cluster policies: towards a participatory approach. European Planning Studies, 9(7), 907-923.

Dillman, D. A. (2007). Mail and internet surveys: The tailored design method, 2nd ed. Mail and internet surveys: The tailored design method, 2nd ed. Hoboken, NJ, US: John Wiley \& Sons Inc.

djazairess. (2015). djazairess. Retrieved December 31, 2018, from https://www.djazairess.com/

Djeflat, A. (2003). The globalised Information Society and its impact on the Europe-Maghreb relationship. Europe and Developing Countries in the Globalized Information Economy: Employment and Distance Education, 87. 
Djeflat, A. (2004). National systems of innovation in the MENA region. World Bank Institute Report, Washington.

Djeflat, A. (2008). Innovation take off through industrial technical centers in Maghreb countries: A missing link in NSI or new opportunity? Georgia Institute of Technology.

Djeflat, A. (2010). Complex innovation systems in Maghreb Countries and the challenges of partnership with Europe. Building Science Technology and Innovation Systems in Africa: Experiences from the Maghreb, 312.

Djeflat, A., Devalan, P., \& Youcef Ettoumi, F. (2007). Evaluation des Politiques et Programmes d'innovation dans le secteur industriel'. Final Report.

Djeflat, A., \& Hamadi, A. (2016). Construction des capacités d'innovation en phase de décollage: cas de l'industrie pharmaceutique en Algérie. International Journal of Economics \& Strategic Management of Business Process (ESMB), 6(Special issue 2016), 20-41.

Dodgson, M., Gann, D., \& Salter, A. (2008). The Management of Technological Innovation Strategy and Practice.

Dolfsma, W., \& Leydesdorff, L. (2011). Innovation systems as patent networks: The Netherlands, India and nanotech. Innovation-Management Policy \& Practice, 13(3), 311-326. Retrieved from \%3CGo

Dosi, G. (1982). Technological paradigms and technological trajectories. A suggested interpretation of the determinants and directions of technical change. Research Policy, 11(3), 147-162. https://doi.org/10.1016/0048-7333(82)90016-6

Dutrénit, G., \& Puchet, M. (2017). Tensions of science, technology and innovation policy in Mexico: analytical models, institutional evolution, national capabilities and governance. Chapters, 205-231. Retrieved from https://www.researchgate.net/publication/295105166

Dutrénit, G., \& Sutz, J. (2014). National Innovation Systems, Social Inclusion and Development. Edward Elgar Publishing. https://doi.org/10.4337/9781782548683

Edler, J. (2013). Review of policy measures to stimulate private demand for innovation. Concepts 
and effects. Compendium of Evidence on the Effectiveness of Innovation Policy Intervention, (13), 44.

Edler, J., \& Fagerberg, J. (2017). Innovation policy: What, why, and how. Oxford Review of Economic Policy, 33(1), 2-23. https://doi.org/10.1093/oxrep/grx001

Edler, J., Gök, A., Cunningham, P., \& Shapira, P. (2016). Introduction: Making sense of innovation policy. In Handbook of Innovation Policy Impact. Edward Elgar Publishing.

Edler, J., Ruhland, S., Hafner, S., Rigby, J., Georghiou, L., Hommen, L., ... Papadakou, M. (2005). Innovation and public procurement. Review of issues at stake. ISI Fraunhofer Institute Systems and Innovation Research, Karlsruhe.

Edquist, C. (1997). Systems of innovation approaches-their emergence and characteristics in Edquist. (L. Pinter/Cassell, Ed.), Systems of Innovation: Technologies, Institutions and Organizations, London: Pinter/Cassell. Routledge. https://doi.org/10.4324/9780203357620

Edquist, Charles. (1997). Systems of innovation approaches-their emergence and characteristics in Edquist. Systems of Innovation: Technologies, Institutions and Organizations. (L. Pinter/Cassell, Ed.). Routledge. https://doi.org/10.4324/9780203357620

Edquist, Charles. (2006). Systems of Innovation: Perspectives and Challenges. journals.co.za. https://doi.org/10.1093/oxfordhb/9780199286805.003.0007

Edquist, Charles. (2011). Design of innovation policy through $n$ diagnostic analysis: identification of systemic problems (or failures). Industrial and Corporate Change, 20(6), 1725-1753. https://doi.org/10.1093/icc/dtr060

Edquist, Charles, \& Hommen, L. (1999). Systems of innovation: Theory and policy for the demand side. Technology in Society, 21(1), 63-79. https://doi.org/10.1016/S0160791X(98)00037-2

Edquist, Charles, \& Johnson, B. (1997). Institutions and organizations in systems of innovation. Systems of innovation.Technologies, institutions, and organizations. Pinter.

Edquist, Charles, \& Zabala-Iturriagagoitia, J. M. (2012). Public Procurement for Innovation as 
Evaluation de la politique d'innovation sur un échantillon d'entreprises algériennes

mission-oriented innovation policy. Research Policy, 41(10), 1757-1769.

Egbetokun, A. A. (2015). Interactive learning and firm-level capabilities in latecomer settings: The Nigerian manufacturing industry. Technological Forecasting and Social Change, 99, 231241. https://doi.org/10.1016/j.techfore.2015.06.040

Ehrnberg, El, \& J, S. (1997). Technological Discontinuities and Incumbents’ Performance: An Analytical Framework. In Systems of Innovation: Technologies, Institutions and Organizations (pp. 318-341).

Ehrnberg, Ellinor, \& Edquist, S. J. (1998). Systems of innovation: Technologies, institutions and organizations. Long Range Planning, 31(2), 333. https://doi.org/10.1016/S00246301(98)90244-8

Eom, B. Y., \& Lee, K. (2010). Determinants of industry-academy linkages and, their impact on firm performance: The case of Korea as a latecomer in knowledge industrialization. Research Policy, 39(5), 625-639. https://doi.org/10.1016/j.respol.2010.01.015

Etzkowitz, H., \& Brisolla, S. N. (1999). Failure and success: The fate of industrial policy in Latin America and South East Asia. Research Policy, 28(4), 337-350. https://doi.org/10.1016/S0048-7333(98)00077-8

Etzkowitz, H., \& Leydesdorff, L. (2000). The dynamics of innovation: From National Systems and “mode 2” to a Triple Helix of university-industry-government relations. Research Policy, 29(2), 109-123. https://doi.org/10.1016/S0048-7333(99)00055-4

Fikirkoca, A., \& Saritas, O. (2012). Foresight for science parks: The case of Ankara University, 24(10), 1071-1085. https://doi.org/10.1080/09537325.2012.723688

Florida, R. (1995). Toward the learning region. Elsevier, 27(5), 527-536. Retrieved from https://www.sciencedirect.com/science/article/pii/001632879500021N

Fornell, C., \& Larcker, D. F. (1981). Evaluating Structural Equation Models with Unobservable Variables and Measurement Error. Journal of Marketing Research, 18(1), 39-50. https://doi.org/10.1177/002224378101800104 
Franco, E., Ray, S., \& Ray, P. K. (2011). Patterns of Innovation Practices of Multinationalaffiliates in Emerging Economies: Evidences from Brazil and India. World Development, 39(7), 1249-1260. https://doi.org/10.1016/j.worlddev.2011.03.003

Frank, A. G., Cortimiglia, M. N., Ribeiro, J. L. D., \& de Oliveira, L. S. (2016). The effect of innovation activities on innovation outputs in the Brazilian industry: Market-orientation vs. technology-acquisition strategies. Research Policy, 45(3), 577-592. https://doi.org/10.1016/j.respol.2015.11.011

Freeman, C. (1987). Technology, policy, and economic performance : lessons from Japan. London [etc.]: Pinter Publishers.

Freeman, C. (1988). Japan: A new national innovation system. Technology and Economy Theory, London: Pinter, 331-348.

Freeman, C. (1995). The 'National System of Innovation' in historical perspective. Cambridge Journal of Economics, 19(1), 5-24. https://doi.org/10.1093/oxfordjournals.cje.a035309

Freeman, C., \& Soete, L. (1997). Development and the diffusion of technology. The Economics of Industrial Innovation, 351-365.

Freidson, E. (1986). Professional powers : a study of the institutionalization of formal knowledge. University of Chicago Press.

Fuchs, G., \& Shapira, P. (2005). Rethinking regional innovation and change: path dependency or regional breakthrough (Vol. 30). Springer Science \& Business Media.

Galende, J., \& de la Fuente, J. M. (2003). Internal factors determining a firm's innovative behaviour. Research Policy, 32(5), 715-736. https://doi.org/10.1016/S0048-7333(02)000823

Galindo, M. A., \& Mendez, M. T. (2014). Entrepreneurship, economic growth, and innovation: Are feedback effects at work? Journal of Business Research, 67(5), 825-829. https://doi.org/10.1016/j.jbusres.2013.11.052

Galli, R., \& Teubal, M. (1997). Paradigmatic shifts in national innovation systems. Systems of 
Evaluation de la politique d'innovation sur un échantillon d'entreprises algériennes

Innovation: Technologies, Institutions and Organizations, 342-370.

Garc, H. V., Cer, O. I., \& Comunitario, D. L. (2020). Innovación incremental en las microempresas del Clúster sectorial lácteo Incremental innovation in micro-enterprises in the dairy sector cluster Inovação incremental em microempresas no cluster do setor de laticínios, 5(08), 20-41. https://doi.org/10.23857/pc.v5i8.1567

Garlsson, B., \& Jacobsson, S. (1991). What makes the automation industry strategic? Economics of Innovation and New Technology, 1(4), 257-269. https://doi.org/10.1080/10438599100000006

Garlsson, B., Jacobsson, S., Carlsson, B., Jacobsson, S., Garlsson, B., Jacobsson, S., ... Jacobsson, S. (1991). What makes the automation industry strategic? Economics of Innovation and New Technology, 1(4), 257-269. https://doi.org/10.1080/10438599100000006

Geisser, S. (1974). A predictive approach to the random effect model. Biometrika, 61(1), 101-107.

George, G., Mcgahan, A. M., \& Prabhu, J. (2012). Innovation for Inclusive Growth: Towards a Theoretical Framework and a Research Agenda. Journal of Management Studies, 49(4), 661-683. https://doi.org/10.1111/j.1467-6486.2012.01048.x

Georghiou, L. (1998). Issues in the evaluation of innovation and technology policy. Evaluation, 4(1), 37-51.

Georghiou, L., Edler, J., Uyarra, E., \& Yeow, J. (2014). Policy instruments for public procurement of innovation: Choice, design and assessment. Technological Forecasting and Social Change, 86, 1-12. https://doi.org/10.1016/j.techfore.2013.09.018

Gibbons, M., \& Johnston, R. (1974). The roles of science in technological innovation. Research Policy, 3(3), 220-242. https://doi.org/10.1016/0048-7333(74)90008-0

Godin, B. (2006). The linear model of innovation: The historical construction of an analytical framework. Science, Technology, \& Human Values, 31(6), 639-667.

Gompers, P. A., \& Lerner, J. (2003). The Determinants of Corporate Venture Capital Success. International Journal of Geriatric Psychiatry (Vol. 18). https://doi.org/10.1002/gps.882 
Bibliographie

Govindarajan, Vijay; Trimble, C. (2013). Reverse Innovation (Abstract). Reverse Innovation, 256. https://doi.org/10.1365/s40112-013-0212-3

Govindarajan, V., \& Ramamurti, R. (2011). Reverse innovation, emerging markets, and global strategy. Global Strategy Journal, 1(3-4), 191-205.

Govindaraju, V., Vijayaraghavan, G. K., \& Pandiyan, V. (2013). Product and process innovation in Malaysian manufacturing: The role of government, organizational innovation and exports. Innovation-Management Policy \& Practice, 15(1), 52-68.

Grimm, R., Fox, C., Baines, S., \& Albertson, K. (2013). Grimm - Social innovation, an answer to contemporary societal challenges? Locating the concept in theory and practice) Social innovation, an answer to contemporary societal challenges? Locating the concept in theory and practice Social innovation, an answ. Innovation: The European Journal of Social Science Research, 26(10), 1351-1610. https://doi.org/10.1080/13511610.2013.848163

Guan, J. C., \& Yan, Y. (2016). Technological proximity and recombinative innovation in the alternative energy field. Research Policy, 45(7), 1460-1473. https://doi.org/10.1016/j.respol.2016.05.002

Guennif, S., \& Ramani, S. V. (2012). Explaining divergence in catching-up in pharma between India and Brazil using the NSI framework. Research Policy, 41(2), 430-441. https://doi.org/10.1016/j.respol.2011.09.005

Guerreri, P., \& Tylecote, A. (1997). Interindustry differences in technical change and national patterns of technological accumulation. In Systems of Innovation: Technologies, Institutions and Organisations (pp. 107-129).

Guerrero, M., Urbano, D., \& Fayolle, A. (2016). Entrepreneurial activity and regional competitiveness: evidence from European entrepreneurial universities. Journal of Technology Transfer, 41(1), 105-131. https://doi.org/10.1007/s10961-014-9377-4

Guerrieri, P., Innovation, A. T.-S. of, \& 1997, U. (n.d.). Interindustry differences in technical change and national patterns of technological accumulation. In researchgate.net.

Guimon, J. (2011). Policies to benefit from the globalization of corporate R\&D: An exploratory 
Evaluation de la politique d'innovation sur un échantillon d'entreprises algériennes

study for EU countries. Technovation, 31(2-3), 77-86. https://doi.org/10.1016/j.technovation.2010.08.001

Gupta, A. (1997). Honey bee network: linking knowledge-rich grassroots innovations. Retrieved from http://vslir.iima.ac.in:8080/jspui/handle/11718/9487

Gupta, H., \& Barua, M. K. (2016). Identifying enablers of technological innovation for Indian MSMEs using best-worst multi criteria decision making method. Technological Forecasting and Social Change, 107, 69-79. https://doi.org/10.1016/j.techfore.2016.03.028

Hair Jr, J. F., Hult, G. T. M., Ringle, C., \& Sarstedt, M. (2016). A primer on partial least squares structural equation modeling (PLS-SEM). Sage Publications.

Haken, H. (1984). Synergetics (Chinese Version Translated by Xishen Xu). Beijing: Atom Press.

Henderson, R. M., \& Clark, K. B. (1990). Architectural Innovation: The Reconfiguration of Existing Product Technologies and the Failure of Established Firms. Administrative Science Quarterly, 35(1), 9. https://doi.org/10.2307/2393549

Henseler, J, Ringle, C. M., \& Sinkovics, R. R. (2009). The use of partial least Squares path modeling in international marketing. In R. R. Sinkovics \& P. N. Ghauri (Eds.) Bingley: Emerald. Advances in International Marketing, 20, 277-320. https://doi.org/10.1016/01678116(92)90003-4

Henseler, Jörg, Dijkstra, T. K., Sarstedt, M., Ringle, C. M., Diamantopoulos, A., Straub, D., ... Calantone, R. J. (2014). Common Beliefs and Reality About Partial Least Squares: Comments on Rönkkö \& Evermann (2013). Organizational Research Methods, 17(2), 182209.

Henseler, Jörg, Ringle, C. M., \& Sinkovics, R. R. (2009). The use of partial least squares path modeling in international marketing. In New challenges to international marketing (pp. 277319). Emerald Group Publishing Limited.

Herrerias, M. J., \& Orts, V. (2013). Capital goods imports and long-run growth: Is the Chinese experience relevant to developing countries? Journal of Policy Modeling, 35(5), 781-797. https://doi.org/10.1016/j.jpolmod.2013.02.006 
Bibliographie

Herstatt, C., Tiwari, R., Buse, S., \& Ernst, D. (2008). India’s National Innovation System: Key Elements and Corporate Perspectives. SSRN Electronic Journal. https://doi.org/10.2139/ssrn.1583699

Hervas-Oliver, J.-L., Albors-Garrigos, J., de-Miguel, B., \& Hidalgo, A. (2012). The role of a firm's absorptive capacity and the technology transfer process in clusters: How effective are technology centres in low-tech clusters? Entrepreneurship \& Regional Development, 24(78), 523-559. https://doi.org/10.1080/08985626.2012.710256

Hildén, M., Jordan, A., \& Rayner, T. (2014). Climate policy innovation: developing an evaluation perspective. Environmental Politics, 23(5), 884-905.

Hirschman, A. O. (1958). The strategy of economic growth. Yale. New Haven.

Howlett, M. (2005). What is a policy instrument? Tools, mixes, and implementation styles. In Designing government: From instruments to governance (McGil-Quee, pp. 31-50). Montréal and Kingston: McGill-Queen’s University Press.

Howlett, M. (2009). Governance modes, policy regimes and operational plans: A multi-level nested model of policy instrument choice and policy design. Policy Sciences, 42(1), 73-89.

Hulland, J. (1999). Use of partial least squares (PLS) in strategic management research: a review of four recent studies. Strategic Management Journal, 20(2), 195-204. https://doi.org/10.1002/(SICI)1097-0266(199902)20:2<195::AID-SMJ13>3.0.CO;2-7

Iizuka, M., \& Katz, J. (2012). Globalization and the changing institution for sustainability: The case of the Salmon farming industry in Chile, (31).

Immelt, Jeffrey R., Vijay Govindarajan, C. T. (2009). How GE Is Disrupting Itself. Harvard Business Review, (87), 56-65.

Intarakumnerd, P., \& Chaoroenporn, P. (2013). The roles of intermediaries in sectoral innovation system in developing countries: public organizations versus private organizations. Asian Journal of Technology Innovation, 21(1), 108-119. https://doi.org/10.1080/19761597.2013.810949 
Intarakumnerd, P., Sunami, A., \& Ueki, Y. (2012). Introduction to the Special Issue on automotive industry in emerging Asian countries. Asian Journal of Technology Innovation, 20, 1-7. https://doi.org/10.1080/19761597.2012.683947

Jaffe, A. B., Newell, R. G., \& Stavins, R. N. (2005). A tale of two market failures: Technology and environmental policy. Ecological Economics, 54(2-3), 164-174.

Jin, J., Wang, Y. D., \& Vanhaverbeke, W. (2014). Patterns of R\&D internationalisation in developing countries: China as a case. International Journal of Technology Management, 64(2-4), 276-302. https://doi.org/10.1504/ijtm.2014.059947

Jones, B., \& Grimshaw, D. (2016). The impact of skill formation policies on innovation. Handbook of Innovation Policy Impact, 108-128.

Jun, S. P., Seo, J. H., \& Son, J. K. (2013). A study of the SME Technology Roadmapping Program to strengthen the R\&D planning capability of Korean SMEs. Technological Forecasting and Social Change, 80(5), 1002-1014. https://doi.org/10.1016/j.techfore.2012.10.022

Justman, M., \& Teubal, M. (1995). Technological infrastructure policy (TIP): creating capabilities and building markets. Research Policy, 24(2), 259-281.

Kamien, I. M., \& Schwartz, L. N. (1975). Market Structure and Innovation: A Survey. Journal of Economic Literature, 13(1), 1-37. Retrieved from http://www.jstor.org/stable/2722211

Kang, K. N., \& Park, H. (2012). Influence of government R\&D support and inter-firm collaborations on innovation in Korean biotechnology SMEs. Technovation, 32(1), 68-78. https://doi.org/10.1016/j.technovation.2011.08.004

Katz, J. (2015). The Latin American Transition from an Inward-Oriented Industrialisation Strategy to a Natural Resource-Based Model of Economic Growth. Institutions and Economies, Volume $\quad 7$ (Issue $\quad 1$ ), $\quad 9-22 . \quad$ Retrieved http://ijie.um.edu.my/index.php/ijie/article/view/4999

Kaufmann, A., \& Todtling, F. (2000). Systems of Innovation in Traditional Industrial Regions: The Case of Styria in a Comparative Perspective. Regional Studies, 34(1), 29-40. https://doi.org/10.1080/00343400050005862 
Khayyat, N. T., \& Lee, J. D. (2015). A measure of technological capabilities for developing countries. Technological Forecasting and Social Change, 92, 210-223. https://doi.org/10.1016/j.techfore.2014.09.003

Kim, Y. (2015). Consumer user innovation in Korea: an international comparison and policy implications. Asian Journal of Technology Innovation, 23(1), 69-86. https://doi.org/10.1080/19761597.2015.1015672

Kleiman, M. A. R., \& Teles, S. M. (2006). Market and non-market failures. In The oxford handbook of public policy.

Kline, S. J., \& Rosenberg, N. (1986). The Positive Sum Strategy: Harnessing Technology For Economic Growth. The Positive Sum Strategy: Harnessing Technology For Economic Growth. National Academy Press. https://doi.org/CTG LANDAU

Knight, F. H. (1921). Risk, Uncertainty and Profit, 1921. Boston and New York, 23(3), 135-157. https://doi.org/10.1017/CBO9781107415324.004

Kotrlik, J., \& Higgins, C. (2001). Organizational research: Determining appropriate sample size in survey research appropriate sample size in survey research. Information Technology, Learning, and Performance Journal, 19(1), 43.

Kuhlmann, S., \& Ordóñez-Matamoros, G. (2017). Research handbook on innovation governance for emerging economies : towards better models.

Kuhlmann, S., Shapira, P., \& Smits, R. E. H. M. (2010). A Systemic Perspective: The Innovation Policy Dance. In The Theory and Practice of Innovation Policy: An International Research Handbook (pp. 1-22). https://doi.org/10.4337/9781849804424.00006

Kwon, K. S. (2011). The co-evolution of universities’ academic research and knowledge-transfer activities: the case of South Korea. Science and Public Policy, 38(6), 493-503. https://doi.org/10.3152/030234211x12960315267930

Laranja, M., Uyarra, E., \& Flanagan, K. (2008). Policies for science, technology and innovation: Translating rationales into regional policies in a multi-level setting. Research Policy, 37(5), 823-835. 
Larédo, P., Köhler, C., \& Rammer, C. (2016). The impact of fiscal incentives for R\&D. Handbook of Innovation Policy Impact, Cheltenham, Edward Elgar, 18-53.

Lee, J. J., \& Yoon, H. (2015). A comparative study of technological learning and organizational capability development in complex products systems: Distinctive paths of three latecomers in military aircraft industry. Research Policy, 44(7), 1296-1313. https://doi.org/10.1016/j.respol.2015.03.007

Lee, S. M., Hwang, T., \& Choi, D. (2012). Open innovation in the public sector of leading countries. Management Decision, 50(1-2), 147-162. https://doi.org/10.1108/00251741211194921

Lennerts, S., Schulze, A., \& Tomczak, T. (2020). The asymmetric effects of exploitation and exploration on radical and incremental innovation performance: An uneven affair. European Management Journal, 38(1), 121-134. https://doi.org/10.1016/j.emj.2019.06.002

Lerner, J., \& Stern, S. (2019). Innovation Policy and the Economy: Introduction to Volume 19. Innovation Policy and the Economy, 19, xi-xiv. https://doi.org/10.1086/699930

Leydesdorff, L. (2000). The triple helix: An evolutionary model of innovations. Research Policy, 29(2), 243-255. https://doi.org/10.1016/S0048-7333(99)00063-3

Li, E. Y., Liao, C. H., \& Yen, H. R. (2013). Co-authorship networks and research impact: A social capital perspective. Research Policy, 42(9), 1515-1530. https://doi.org/10.1016/j.respol.2013.06.012

Lohmöller, J.-B. (1989). Predictive vs. structural modeling: Pls vs. ml. In Latent variable path modeling with partial least squares (pp. 199-226). Springer.

Lohmöller, J.-B. (2013). Latent variable path modeling with partial least squares. Springer Science \& Business Media.

Lundvall, B.-A. (1985). Product innovation and user-producer interaction. Aalborg University Press. Retrieved from http://www.forskningsdatabasen.dk/en/catalog/2389383064

Lundvall, B.-A. (1992). National systems of innovation: towards a theory of innovation and 
Bibliographie

interactive learning. National Systems of Innovation: Towards a Theory of Innovation and

Interactive Learning. Pinter Publishers. Retrieved from http://www.forskningsdatabasen.dk/en/catalog/2389367346

Lundvall, B.-Å. (1992). User-producer relationships, national systems of innovation and internationalisation. In National systems of innovation: Towards a theory of innovation and interactive learning (pp. 45-67). Frances Pinter Publishers Ltd.

Lundvall, B.-Å. (2006). Interactive learning, social capital and economic performance. Advancing Knowledge and the Knowledge Economy, 63-74.

Lundvall, B.-Å. (2016). The Learning Economy and the Economics of Hope. Research Series 31,. https://doi.org/http://dx.doi.org/10.1016/B978-0-7506-7009-8.50019-7

Lundvall, B.-Å., \& Borrás, S. (2006). Science, Technology, and Innovation Policy. The Oxford Handbook of Innovation. Oxford University Press. https://doi.org/10.1093/oxfordhb/9780199286805.003.0022

Lundvall, B.-å. (1998). Why study national systems and national styles of innovation? Technology Analysis \& Strategic Management, 10(4), 403-422.

Lundvall, B.-äke, \& Johnson, B. (1994). The Learning Economy. Journal of Industry Studies, 1(2), 23-42. https://doi.org/10.1080/13662719400000002

Lundvall, B. A. (2004). Introduction to "Technological infrastructure and international competitiveness” by Christopher Freeman. Industrial and Corporate Change, 13(3), 531539. https://doi.org/10.1093/icc/13.3.531

Magro, E., \& Wilson, J. R. (2013). Complex innovation policy systems: Towards an evaluation mix. Research Policy, 42(9), 1647-1656.

Malerba, F, \& Orsenigo, L. (1995). Schumpeterian patterns of innovation. Cambridge Journal of Economics, 19, 47-65. https://doi.org/10.1093/oxfordjournals.cje.a035308

Malerba, Franco;, \& Orsenigo, L. (1990). Technological Regimes and Patterns of Innovation: A Theoretical and Empirical Investigation of the Italian Case. In Evolving Technology and 
Evaluation de la politique d'innovation sur un échantillon d'entreprises algériennes

Market Structure - Studies in Schumpeterian Economics (pp. 283-305).

Mansfield, E. (1988). The Speed and Cost of Industrial Innovation in Japan and the United States:

External vs. Internal Technology. Management Science, 34(10), 1157-1168. https://doi.org/10.1287/mnsc.34.10.1157

Marcelle, G. M. (2004). Technological learning : a strategic imperative for firms in the developing world. Edward Elgar Pub.

Marcelle, G. M. (2017). Science, technology and innovation policy that is responsive to innovation performers. In Research Handbook on Innovation Governance for Emerging Economies (pp. 59-86). Retrieved from https://ideas.repec.org/h/elg/eechap/15643_2.html

Mazzucato, M., \& Penna, C. (2015). Mission-oriented finance for innovation: New ideas for investment-led growth (Policy Net). Policy Network and Rowman \& Littlefield International.

McCool, D. (1995). Public policy theories, models, and concepts: an anthology. Prentice Hall. Retrieved from https://books.google.es/books?id=8290AQAAIAAJ

McCullagh, P., \& Meyer, K. N. (1997). Learning versus correct models: Influence of model type on the learning of a free-weight squat lift. Research Quarterly for Exercise and Sport, 68(1), 56-61.

McKelvey, M. (1991). How do national systems of Innovation Differ? Edward Elgar Publishers.

Meesapawong, P., Rezgui, Y., \& Li, H. J. (2014). Planning innovation orientation in public research and development organizations: Using a combined Delphi and Analytic Hierarchy Process approach. Technological Forecasting and Social Change, 87, 245-256. https://doi.org/10.1016/j.techfore.2013.12.023

Meyer-Krahmer, F. (1997). Science-based technologies and interdisciplinarity: Challenges for firms and policy. Edquist, C., Systems of Innovation, Pinter, London and Washington, 298317.

Montresor, S., \& Vezzani, A. (2016). Intangible investments and innovation propensity: Evidence 
Bibliographie

from the Innobarometer 2013. Industry and Innovation, 23(4), 331-352.

https://doi.org/10.1080/13662716.2016.1151770

Morris, M., Kaplinsky, R., \& Kaplan, D. (2012). “One thing leads to another”-Commodities, linkages and industrial development. Resources Policy, 37(4), 408-416. https://doi.org/10.1016/j.resourpol.2012.06.008

Mothe, J., \& Paquet, G. (1998). Local and Regional Systems of Innovation. Springer US.

Moulaert, F., Martinelli, F., González, S., \& Swyngedouw, E. (2007). Introduction: social innovation and governance in European cities: urban development between path dependency and radical innovation. Sage Publications Sage UK: London, England.

Mowery, D. (1997). The Bush report after fifty years-Blueprint or relic. Science for the 21, 21.

Nasreddine, A. (2017). The Algerian pharmaceutical market; specifics and characteristics, 1, 5780.

Navarro, M., Valdaliso, J. M., Aranguren, M. J., \& Magro, E. (2014). A holistic approach to regional strategies: The case of the Basque Country. Science and Public Policy, 41(4), 532547. https://doi.org/10.1093/scipol/sct080

Nelson, A., Earle, A., Howard-Grenville, J., Haack, J., \& Young, D. (2014). Do innovation measures actually measure innovation? Obliteration, symbolic adoption, and other finicky challenges in tracking innovation diffusion. Research Policy, 43(6), 927-940. https://doi.org/10.1016/j.respol.2014.01.010

Nelson, R. (1993). National innovation systems: a comparative analysis. Oxford University Press on Demand.

Nelson, R. R. (1988). Institutions supporting technical change in the United States. Technical Change and Economic Theory, 312-329.

NELSON, R. R. (1998). The Co-evolution of Tichnology, Industrial Strurture, and Supporting. Technology, Organization, and Competitiveness: Perspectives on Industrial and Corporate Change, 319. 
Nelson, R. R. R., \& Winter, S. G. (1982). An Evolutionary Theory of Economic Change (Cambridge, Massachusetts and London, Belknap Press of Harvard University Press).

Nelson, R. R., \& Rosenberg, N. (1993). Technical innovation and national systems. National Innovation Systems: A Comparative Analysis, 1, 3-21.

Nelson, R. R., \& Winter, S. G. (1977). In search of useful theory of innovation. Research Policy, 6(1), 36-76.

NEPAD. (2014). African innovation outlook II. Retrieved October 9, 2018, from https://www.nepad.org/

Nishimura, J., \& Okamuro, H. (2011). Subsidy and networking: The effects of direct and indirect support programs of the cluster policy. Research Policy, 40(5), 714-727. https://doi.org/10.1016/j.respol.2011.01.011

North, D. C. (1990). Institutions, Institutional Change and Economic Performance (Political Economy of Institutions and Decisions).

North, D. C. (1991). Institutions, ideology, and economic performance. Cato J., 11, 477.

North, D. C. (1994). Economic performance through time. The American Economic Review, 84(3), 359-368.

North, D. C., Perspectives, D. N.-J. of economic, 1991, undefined, (1986-1998), D. N.-T. J. of E. P., 1991, undefined, Perspectives, D. N.-J. of economic, \& 1991, undefined. (1991). Institutions. Journal of Economic Perspectives, 5(1), 97-112. https://doi.org/10.1257/jep.5.1.97

North, D., jpe, G. B.-, Institutions, D. N.-, 2005, undefined, North, D., Institutions, D. N.-, ... North, D. (1990). INSTITUTIONS, INSTITUTIONAL CHANGE AND ECONOMIC PERFORMANCE. Kim.Sumdu.Edu.Ua. Retrieved from http://www.angelfire.com/ak5/opni/biblioteka/north.pdf

Nunnally, J. (1967). Psychometric theory. Retrieved from http://www.sidalc.net/cgibin/wxis.exe/?IsisScript=UACHBC.xis\&method=post\&formato=2\&cantidad=1\&expresion= 
Bibliographie

$\mathrm{mfn}=038224$

Nunnally, J. C., \& Bernstein, I. H. (1994). Psychometric theory. McGraw-Hill.

Ocde. (2000). Manuel d’Oslo. Creativewallonia.Be, 103. https://doi.org/10.1787/9789264013124$\mathrm{fr}$

Ocde. (2005). Manuel d'Oslo: principes directeurs pour le recueil et l'interprétation des données sur l'innovation. OECD Publishing.

OCDE. (2016). Manuel de Frascati 2015. Online. Éditions OCDE. https://doi.org/10.1787/9789264257252-fr

Ocde, OCDE et Eurostat, \& Ocde. (2005). Manuel d'Oslo - Principes directeurs pour le recueil et l'interprétation des données sur l'innovation,. OECD Publishing. https://doi.org/10.1787/9789264013124-fr

Orozco, J. (2017). Innovation and inclusive growth in the small-scale fishing sector of the Fonseca Gulf, Central America. Chapters, 292-315. Retrieved from https://ideas.repec.org/h/elg/eechap/15643_10.html

Padilla-Perez, R., \& Gaudin, Y. (2014). Science, technology and innovation policies in small and developing economies: The case of Central America. Research Policy, 43(4), 749-759. https://doi.org/10.1016/j.respol.2013.10.011

Papaioannou, T., Watkins, A., Mugwagwa, J., \& Kale, D. (2016). To Lobby or to Partner? Investigating the Shifting Political Strategies of Biopharmaceutical Industry Associations in Innovation Systems of South Africa and India. World Development, 78, 66-79. https://doi.org/10.1016/j.worlddev.2015.10.017

Parida, V., Westerberg, M., \& Frishammar, J. (2012). Inbound open innovation activities in high-tech SMEs: the impact on innovation performance. Journal of Small Business Management, 50(2), 283-309.

Park, J., Jeong, S., Yoon, Y., \& Lee, H. (2015). The evolving role of collaboration in developing scientific capability: Evidence from Korean government-supported research institutes. 
Evaluation de la politique d'innovation sur un échantillon d'entreprises algériennes

Science and Public Policy, 42(2), 255-272. https://doi.org/10.1093/scipol/scu041

Patel, P., \& Pavitt, K. (1991). Europe’s technological performance.

Paunov, C., \& Rollo, V. (2016). Has the Internet Fostered Inclusive Innovation in the Developing World? World Development, 78, 587-609. https://doi.org/10.1016/j.worlddev.2015.10.029

Pavitt, K. (1984). Sectoral patterns of technical change: Towards a taxonomy and a theory. Research Policy, 13(6), 343-373. https://doi.org/10.1016/0048-7333(84)90018-0

Payumo, J., Gang, Z., Pulumbarit, E., Jones, K., Maredia, K., \& Grimes, H. (2012). Managing intellectual property and technology commercialization: Comparison and analysis of practices, success stories and lessons learned from public research universities in developing Asia. Innovation-Management Policy \& Practice, 14(4), 478-494.

Peck, J., \& Theodore, N. (2010). Mobilizing policy: Models, methods, and mutations. Geoforum, 41(2), 169-174. https://doi.org/10.1016/j.geoforum.2010.01.002

Peltoniemi, M. (2005). Business ecosystem: A conceptual model of an organisation population from the perspectives of complexity and evolution.

Peltoniemi, Mirva. (2006). Preliminary theoretical framework for the study of business ecosystems. Emergence: Complexity \& Organization. https://doi.org/10.1007/s11252-0060005-4

Pezeshkan, A., Smith, A., Fainshmidt, S., \& Sedeh, A. A. (2016). National business systems and firm innovation: A study of developing economies. Journal of Business Research, 69(11), 5413-5418. https://doi.org/10.1016/j.jbusres.2016.04.147

Porter, M. E. (1985). The Competitive Advantage: Creating and Sustaining Superior Performance. Retrieved from https://www.hbs.edu/faculty/Pages/item.aspx?num=193

Publishing, O. (2006). La Mesure des Activités Scientifiques Et Technologiques Manuel D’Oslo: Principes Directeurs Pour Le Recueil Et L'Interprétation des Données Sur L'Innovation (3E Édition). Organization for Economic Cooperation \& Development. Retrieved from http://dx.doi.org/10.1787/9789264013124-fr 
Radas, S., Anit, I. D., Tafro, A., \& Wagner, V. (2015). The effects of public support schemes on small and medium enterprises. Technovation, 38, 15-30. https://doi.org/10.1016/j.technovation.2014.08.002

Radjou, N., Prabhu, J. C., \& Ahuja, S. (2012). Jugaad innovation: think frugal, be flexible, generate breakthrough growth. https://doi.org/10.1108/SAJGBR-03-2013-0014

Radosevic, S. (1994). Strategic technology policy for Eastern Europe. Economic Systems, 18(2), 87-116.

Radosevic, S. (1999). Transformation of science and technology systems into systems of innovation in central and eastern Europe: the emerging patterns and determinants. Structural Change and Economic Dynamics, 10(3-4), 277-320.

Ramlogan, R., \& Rigby, J. (2014). The Impact and Effectiveness of Entrepreneurship Policy.

Rasiah, R., Gopal, V., \& Sanjivee, P. (2013). Export and innovation in Cambodian clothing manufacturing firms. Asian Journal of Technology Innovation, 21(2), 305-316. https://doi.org/10.1080/19761597.2013.866312

RC, L., \& WM, C. (1987). R\&D Appropriability, Opportunity Market Structure: New Evidence on Some Schumpeterian Hypotheses: American Economic Review. 研究 技術 計画, 2(4), 478479.

Rennkamp, B. (2017). Out of sync: innovation policy and theory in unequal societies. Chapters, 87-118. Retrieved from https://ideas.repec.org/h/elg/eechap/15643_3.html

Rigby, J. (2016). 12. The impact of pre-commercial procurement on innovation. Handbook of Innovation Policy Impact, 382.

Rosenberg, N. (1982). Inside the Black Box: Technology and Economics. https://doi.org/loc?

Rothwell, R, \& Zegveld, W. (1985). Reindustrialization and Technology.

Rothwell, Roy. (1980). The impact of regulation on innovation: some US data. Technological Forecasting and Social Change, 17(1), 7-34.

Rothwell, Roy. (1992). Successful industrial innovation: critical factors for the 1990s. $R \& D$ 
Evaluation de la politique d'innovation sur un échantillon d'entreprises algériennes

Management, 22(3), 221-240. https://doi.org/10.1111/j.1467-9310.1992.tb00812.x

Rothwell, Roy, \& Gardiner, P. (1988). Re-innovation and robust designs: Producer and user benefits. Journal of Marketing Management, 3(3), 372-387. https://doi.org/10.1080/0267257X.1988.9964053

Rubalcaba, L., Aboal, D., \& Garda, P. (2016). Service Innovation in Developing Economies: Evidence from Latin America and the Caribbean. Journal of Development Studies, 52(5), 607-626. https://doi.org/10.1080/00220388.2015.1093118

Salazar, M. (2017). The Colombian system of science, technology and innovation in transition: how governance is being affected. In Research Handbook on Innovation Governance for Emerging Economies: Towards Better Models (pp. 232-264). Edward Elgar Publishing. https://doi.org/10.4337/9781783471911

Samara, E., Georgiadis, P., \& Bakouros, I. (2012). The impact of innovation policies on the performance of national innovation systems: A system dynamics analysis. Technovation, 32(11), 624-638. https://doi.org/10.1016/j.technovation.2012.06.002

Sampat, B. N. (2012). Mission-oriented biomedical research at the NIH. Research Policy, 41(10), 1729-1741.

Sanderson, I. (2002). Evaluation, policy learning and evidence-based policy making. Public Administration, 80(1), 1-22.

Santé, O. mondiale de la. (1997). La libéralisation du secteur pharmaceutique en Algérie. Apps. Who.Int. Retrieved from http://apps.who.int/medicinedocs/collect/medicinedocs/pdf/s2213f/s2213f.pdf

Saunders, M., Lewis, P., \& Thornhill, A. (2008). Research Methods for Students. Research methods for business students. https://doi.org/10.1007/s13398-014-0173-7.2

Saxenian, A. (1994). Regional Advantage: Culture and Competition in Silicon Valley and Route 128 (Cambrigde, MA: Harvard Univ.).

Schmidt, T., \& Rammer, C. (2007). Non-technological and Technological Innovation: Strange 
Bibliographie

Bedfellows? ZEW research. https://doi.org/10.2139/ssrn.1010301

Schumpeter, J. A. (1934). The Schumpttr: Theory Economic Development.

Schumpeter, J. A. C. N.-J. or A. B. R. R. H. . S. (1942). Capitalism, Socialism, and Democracy (First edit). Retrieved from http:/www.worldcat.org/title/capitalism-socialism-anddemocracy/oclc/30488029

Scott, W. R. (1991). Unpacking Institutional Arguments. S. 164-182 in: WB Powell, PJ DiMaggio (Hrsg.): The New Institutionalism in Organizational Analysis. Chicago, London: University of Chicago Press. Google Scholar.

Sedkaoui, S. (2016). Les obstacles au processus d'innovation. Etude empirique basée sur un échantillon d'entreprises pharmaceutiques algériennes. Marché et organisations (Vol. 2). https://doi.org/10.3917/maorg.026.0121

Sesay, B., Yulin, Z., \& Wang, F. (2018). Does the national innovation system spur economic growth in Brazil, Russia, India, China and South Africa economies? Evidence from panel data. South African Journal of Economic and Management Sciences, 21(1), 1-12.

Shapira, P., \& Youtie, J. (2016). The impact of technology and innovation advisory services. In Handbook of Innovation Policy Impact. Edward Elgar Publishing.

Sharif, M. N. (2012). Technological innovation governance for winning the future. Technological Forecasting and Social Change, 595-604. https://doi.org/10.1016/j.techfore.2011.12.004

Sharif, N. (2006). Research policy. Research Policy (Vol. 35). Elsevier Science Publishers B.V. (North-Holland). https://doi.org/10.1016/j.respol.2006.04.001

Smith, Adam. (1976). An inquiry into the nature and causes of the wealth of nations (ed. RH Campbell, AS Skinner, and WB Todd). Retrieved from https://philpapers.org/rec/SMIAII-3/

Smith, Adrian, Fressoli, M., \& Thomas, H. (2014). Grassroots innovation movements: Challenges and contributions. Journal of Cleaner Production, 63, 114-124. https://doi.org/10.1016/j.jclepro.2012.12.025 
Smith, K. (1997). Economic infrastructures and innovation systems. Systems of Innovation: Technologies, Institutions and Organisations, 86-106.

Smits, R., \& Kuhlmann, S. (2004). The rise of systemic instruments in innovation policy. International Journal of Foresight and Innovation Policy, 1(1-2), 4-32.

Smorodinskaya, N., Russell, M. G., Katukov, D., \& Still, K. (2017). Innovation Ecosystems vs. Innovation Systems in Terms of Collaboration and Co-creation of Value.

Spielman, D. J., \& Ma, X. (2016). Private Sector Incentives and the Diffusion of Agricultural Technology: Evidence from Developing Countries. Journal of Development Studies, 52(5), 696-717. https://doi.org/10.1080/00220388.2015.1081171

Srholec, M. (2011). A multilevel analysis of innovation in developing countries. Industrial and Corporate Change. https://doi.org/10.1093/icc/dtr024

Srinivas, S., \& Sutz, J. (2008a). Developing countries and innovation: Searching for a new analytical approach, 30(2), 129-140. https://doi.org/10.1016/j.techsoc.2007.12.003

Srinivas, S., \& Sutz, J. (2008b). Developing countries and innovation: Searching for a new analytical approach. Technology in Society, 30(2), 129-140. https://doi.org/10.1016/j.techsoc.2007.12.003

Stavins, R. N. (1996). Correlated uncertainty and policy instrument choice. Journal of Environmental Economics and Management, 30(2), 218-232.

Stek, \& van Geenhuizen, M. S. (2016). The influence of international research interaction on national innovation performance: A bibliometric approach. Technological Forecasting and Social Change, 110, 61-70. https://doi.org/10.1016/j.techfore.2015.09.017

Stiglitz, J. E. (2000). Formal and informal institutions. Social Capital: A Multifaceted Perspective, 59-68.

Stone, M. (1974). Cross-validatory choice and assessment of statistical predictions. Journal of the Royal Statistical Society: Series B (Methodological), 36(2), 111-133.

Strambach, S. (2008). Path dependency and path plasticity: the co-evolution of institutions and 
innovation-the German customized business software industry. Working Papers on Innovation and Space.

Su, Y.-S., \& Wu, F.-S. (2015). Regional systems of biotechnology innovation - The case of Taiwan. Technological Forecasting and Social Change, 100, 96-106. https://doi.org/10.1016/J.TECHFORE.2015.10.002

Sung, T. K. (2015). The creative economy in global competition tr. Technological Forecasting \& Social Change, 96, 89-91. https://doi.org/10.1016/j.techfore.2015.04.003

Suominen, A., Seppänen, M., \& Dedehayir, O. (2016). Innovation Systems and Ecosystems: a Review and Synthesis. ISPIM Innovation Conference: Blending Tomorrow's Innovation Vintage, (June), 2016.

Symes, C., McIntyre, J., \& Barnett, R. (2002). Working Knowledge. Open University Press.

Tassey, G. (1991). The functions of technology infrastructure in a competitive economy. Research Policy, 20(4), 345-361.

TAYLOR, F. W. (1914). SCIENTIFIC MANAGEMENT. The Sociological Review, 7 a(3), 266269. https://doi.org/10.1111/j.1467-954X.1914.tb02387.x

Tenenhaus, M., Vinzi, V. E., Chatelin, Y.-M., \& Lauro, C. (2005). PLS path modeling. Computational Statistics \& Data Analysis, 48(1), 159-205.

Teubal, M. (1998). Enterprise restructuring and embeddedness: An innovation systems and policy perspective. Centre for Research on Innovation and Competition, University of Manchester.

Teubal, M. (2002). What is the systems perspective to Innovation and Technology Policy (ITP) and how can we apply it to developing and newly industrialized economies? Journal of Evolutionary Economics, 12(1-2), 233-257.

Tey, L. S., \& Idris, A. (2012). Cultural fit, knowledge transfer and innovation performance: a study of Malaysian offshore international joint ventures. Asian Journal of Technology Innovation, 20(2), 201-218. https://doi.org/10.1080/19761597.2012.727698

Thorsteinsdóttir, H. (2007). The Role of the Health System in Health Biotechnology in Developing 
Evaluation de la politique d'innovation sur un échantillon d'entreprises algériennes

Countries. Technology Analysis \& Strategic Management, 19(5), 659-675. https://doi.org/10.1080/09537320701521432

Tirole, J. (2015). Market failures and public policy. American Economic Review, 105(6), 16651682.

Tödtling, F., \& Trippl, M. (2005a). One size fits all?: Towards a differentiated regional innovation policy approach. Research Policy, 34(8), 1203-1219. https://doi.org/10.1016/j.respol.2005.01.018

Tödtling, F., \& Trippl, M. (2005b). One size fits all? Research Policy, 34(8), 1203-1219. https://doi.org/10.1016/j.respol.2005.01.018

Toivanen, H. (2014). The shift from theory to innovation: the evolution of Brazilian research frontiers 2005-2011. Technology Analysis and Strategic Management, 26(1), 105-119. https://doi.org/10.1080/09537325.2013.850160

Tseng, C. H., \& Chen, L. T. (2014). Determinants of subsidiary’s technological capability Examining the roles of subsidiary-local supplier linkage, 29(5), 374-386. https://doi.org/10.1108/JBIM-06-2012-0094

Turró, A., Urbano, D., \& Peris-Ortiz, M. (2014). Culture and innovation: The moderating effect of cultural values on corporate entrepreneurship. Technological Forecasting and Social Change, 88, 360-369. https://doi.org/10.1016/j.techfore.2013.10.004

Tushman, M. L., \& Anderson, P. (1986). Technological Discontinuities and Organizational Environments. Administrative Science Quarterly, 439. https://doi.org/10.2307/2392832

Utterback, J. (1994). Mastering the dynamics of innovation: how companies can seize opportunities in the face of technological change. Retrieved from https://dl.acm.org/citation.cfm?id=189474

Uyarra, E. (2016). 11. The impact of public procurement of innovation. Handbook of Innovation Policy Impact, 355. 
Uyarra, E., \& Ramlogan, R. (2012). The effects of cluster policy on innovation. Compendium of Evidence on the Effectiveness of Innovation Policy Intervention, Manchester Institute of Innovation Research.

Uyarra, E., \& Ramlogan, R. (2016). The impact of cluster policy on innovation. Handbook of Innovation Policy Impact, 196-225.

Weerawardena, J., O’Cass, A., \& Julian, C. (2006). Does industry matter? Examining the role of industry structure and organizational learning in innovation and brand performance. Journal of Business Research, 59(1), 37-45. https://doi.org/10.1016/J.JBUSRES.2005.02.004

Werts, C. E., Linn, R. L., \& Jöreskog, K. G. (1974). Intraclass reliability estimates: Testing structural assumptions. Educational and Psychological Measurement, 34(1), 25-33.

WINTER, S. (1998). Knowledge and Competence as Strategic Assets. In The Strategic Management of Intellectual Capital (pp. 165-187). https://doi.org/10.1016/B978-0-75069850-4.50013-0

Wold, H. (1974). Causal flows with latent variables: Partings of the ways in the light of NIPALS modelling. European Economic Review. [Amsterdam]: https://doi.org/10.1016/00142921(74)90008-7

Wold, H. (1985). Partial least squares. S. Kotz and NL Johnson (Eds.), Encyclopedia of statistical sciences (vol. 6). Wiley, New York.

Wold, Herman. (1982). Soft modeling: the basic design and some extensions. Systems under Indirect Observation, 2, 343.

Wong, C. Y., \& Goh, K. L. (2015). Catch-up models of science and technology: A theorization of the Asian experience from bi-logistic growth trajectories. Technological Forecasting and Social Change. https://doi.org/10.1016/j.techfore.2014.02.005

Xie, X. M., Wu, Y. H., \& Ma, G. X. (2016). Driving forces of industrial clusters towards innovative clusters: accelerating the innovation process. Asian Journal of Technology Innovation, 24(2), 161-178. https://doi.org/10.1080/19761597.2016.1196009 
Evaluation de la politique d'innovation sur un échantillon d'entreprises algériennes

Xu, Q., Chen, J., Xie, Z., Liu, J., Zheng, G., \& Wang, Y. (2006). Total Innovation Management: a novel paradigm of innovation management in the 21st century. The Journal of Technology Transfer, 32(1-2), 9-25. https://doi.org/10.1007/s10961-006-9007-x

Yun, S., \& Lee, J. (2013). An innovation network analysis of science clusters in South Korea and Taiwan. Asian Journal of Technology Innovation, 21(2), 277-289. https://doi.org/10.1080/19761597.2013.866310

Zahra, S. A., \& George, G. (2002). Absorptive Capacity: A Review, Reconceptualization, and Extension. Academy of Management Review, 27(2), 185-203. https://doi.org/10.5465/amr.2002.6587995

Zhou, Y., \& Minshall, T. (2014). Building global products and competing in innovation: the role of Chinese university spin-outs and required innovation capabilities. International Journal of Technology Management, 64(2/3/4), 180. https://doi.org/10.1504/IJTM.2014.059929

Zysman, J. (1983). Governments, markets, and growth: financial systems and the politics of industrial change. 


\subsection{Annexe}

7.1.1 Annexe 1 Le guide de l'interview

https://www2.ingenio.upv.es/es/Sondage_sur_la_politique_d\%27innovation

Information générale sur l'entreprise

Nom:

Fonction:

Organisation:

Téléphone:

E-mail:

Nom de l'entreprise

Adresse

Code postal

Activité principale

Date de création :

Les acteurs de l'innovation

1. Avec qui vous collaborer dans le cadre de l'innovation ? et comment ?

\begin{tabular}{|l|l|l|}
\hline 1.1 & Autres entreprises de votre groupe d'entreprises & \\
\hline 1.2 & Fournisseurs d'équipements, de matériaux, de composants ou de logiciels & \\
\hline 1.3 & Des clients ou fournisseur du secteur public & \\
\hline 1.4 & Des clients ou fournisseurs du secteur privé & \\
\hline 1.5 & Concurrents ou autres entreprises de votre secteur & \\
\hline 1.6 & Consultants ou laboratoires commerciaux & \\
\hline 1.7 & Universités & \\
\hline 1.8 & Gouvernements, instituts de recherche publics ou privés & \\
\hline
\end{tabular}


Instrument politique

2. Quel programme du gouvernement d'appui à l'innovation vous utiliser pour innover ?

\begin{tabular}{|l|l|l|}
\hline 2.1 & Les programmes d'encouragement fiscal & \\
\hline 2.2 & $\begin{array}{l}\text { Crédit d'impôt du gouvernement pour la recherche et le dévelop- } \\
\text { pement ou les dépenses en immobilisations. }\end{array}$ & \\
\hline 2.3 & $\begin{array}{l}\text { Les programmes de subventions pour le développement et la } \\
\text { commercialisation de la propriété intellectuelle }\end{array}$ & \\
\hline 2.4 & Les programmes de formation et d'embauche & \\
\hline 2.5 & Les marchés publics & \\
\hline 2.6 & L'accès aux installations, & \\
\hline 2.7 & Les incitatifs à l'exportation, & \\
\hline 2.8 & L'assistance technique, & \\
\hline 2.9 & L'information sur le marché . & \\
\hline
\end{tabular}

Demande d'innovation

3. Quelle activité utiliser-vous pour promouvoir l'innovation?

\begin{tabular}{|c|c|}
\hline 3.1 & R \& D interne \\
\hline 3.2 & R \& D externe \\
\hline 3.3 & Acquisitions de machines, équipements, logiciels et bâtiments \\
\hline 3.4 & $\begin{array}{l}\text { Acquisitions des connaissances existantes par d'autres entreprises } \\
\text { ou organisations }\end{array}$ \\
\hline 3.5 & Formations pour des activités innovantes \\
\hline 3.6 & $\begin{array}{l}\text { Introductions sur le marché des innovations (les études de marché } \\
\text { et la publicité de lancement) }\end{array}$ \\
\hline 3.7 & $\begin{array}{l}\text { Conception : Activités internes ou sous-traitées pour modifier la } \\
\text { forme , l'apparence ou la facilité d'utilisation de biens ou de ser- } \\
\text { vices }\end{array}$ \\
\hline 3.8 & Licences ou achat d'un brevet, \\
\hline 3.9 & Utilisation avancée de la technologie \\
\hline
\end{tabular}


Evaluation de la politique d'innovation sur un échantillon d'entreprises algériennes

\section{Les résultats d'innovation}

4. Vous avez quel type d'innovation ?

\begin{tabular}{|l|l|}
\hline 4.1 & Innovation en matière de biens : produits \\
\hline 4.2 & Innovations de service \\
\hline 4.3 & Innovations dans le processus \\
\hline 4.4 & Innovations organisationnelles \\
\hline 4.5 & Innovations marketing \\
\hline 4.6 & Brevets, licence, propriété intellectuelle \\
\hline
\end{tabular}

\section{Les barrières à l'innovation}

5. Quel type d'obstacle avez-vous pour innover ?

\begin{tabular}{|l|l|}
\hline 5.1 & Manque de financement interne pour l'innovation \\
\hline 5.2 & Manques de crédit ou de capital-investissement \\
\hline 5.3 & Manques d'employés qualifiés dans votre entreprise \\
\hline 5 & $\begin{array}{l}\text { Difficultés à obtenir des subventions } \\
\text { gouvernementales ou des subventions } \\
\text { pour l'innovation }\end{array}$ \\
\hline 5.5 & Manques de partenaires de collaboration \\
\hline 5.6 & Demande du marché incertain pour vos idées d'innovations \\
\hline 5.7 & Trop de concurrence dans votre marché \\
\hline 5.7 & Tailles du marché \\
\hline 5.8 & Manques de compétence \\
\hline 5.9 & Trouver et conclure des accords avec des collaborateurs externes \\
\hline 5.10 & Incertitudes et risque \\
\hline 5.11 & Politique de concurrence réglementaire ou gouvernementale \\
\hline 5.12 & Protections de la propriété intellectuelle \\
\hline
\end{tabular}


6. Quels programmes gouvernementaux vous paraissent adéquats pour surmonter les obstacles à l'innovation?

7. Quelle mesure vous souhaiter surmonter pour le développement dans l'entreprise ?

8. Quels types de facilités vous souhaiter pour surmonter les obstacles à l'innovation ?

9. Quels sont les obstacles rencontrés pour adopter ou utiliser des technologies avancées ?

\begin{tabular}{|c|c|}
\hline 9.1 & Pas convaincu de l'avantage économique \\
\hline 9.2 & Difficulté à obtenir un financement \\
\hline 9.3 & Coût élevé des technologies de pointe \\
\hline 9.4 & Investissement non nécessaire pour la poursuite des opérations \\
\hline 9.5 & $\begin{array}{l}\text { Manque de compétences techniques nécessaires pour soutenir ce type } \\
\text { d'investissement }\end{array}$ \\
\hline 9.6 & La culture organisationnelle trop rigide \\
\hline 9.7 & Décisions prises par des sociétés mères, affiliées ou filiales \\
\hline 9.8 & $\begin{array}{l}\text { Manque de support technique ou de services (de consultants ou de } \\
\text { vendeurs) }\end{array}$ \\
\hline 9.9 & Manque d'information sur la technologie de pointe \\
\hline 9.10 & $\begin{array}{l}\text { Difficulté à intégrer de nouvelles technologies de pointe avec les systèmes, } \\
\text { les normes et les processus existants }\end{array}$ \\
\hline 9.11 & Ne s'applique pas aux activités de cette entreprise \\
\hline
\end{tabular}

